\title{
Resolução numérica de EDPs utilizando ondaletas harmônicas
}

\author{
Pedro da Silva Peixoto \\ DisSERTAÇÃO APRESENTADA \\ $\mathrm{AO}$ \\ Instituto De Matemática e Estatística \\ DA \\ Universidade De SÃo Paulo \\ PARA \\ OBTENÇÃO DO TÍTULO \\ $\mathrm{DE}$ \\ Mestre em CiênCias
}

Programa: Matemática Aplicada

Orientador: Prof. Dr. Saulo Rabello Maciel de Barros

Durante o desenvolvimento deste trabalho o autor recebeu auxílio financeiro do CNPq

São Paulo, Junho de 2009 


\section{Resolução numérica de EDPs utilizando ondaletas harmônicas}

Este exemplar corresponde à redação

final da dissertação devidamente corrigida

e defendida por Pedro da Silva Peixoto e aprovada pela Comissão Julgadora.

Banca Examinadora:

- Prof. Dr. Saulo Rabello Maciel de Barros (Presidente) - IME-USP

- Prof. Dr. José Carlos Simon de Miranda - IME-USP

- Profa. Dra. Sônia Maria Gomes - UNICAMP 


\section{Agradecimentos}

Ao meu orientador, Saulo, que teve muita paciência e competência para me guiar durante o mestrado.

Aos professores do IME, que me ajudaram a entender um pouco melhor este vasto mundo da matemática.

Aos colegas do IME, que discutiram problemas matemáticos comigo quando foi necessário, e também participaram na descontração dos momentos de folga.

Ao $\mathrm{CNPq}$, pelo suporte financeiro.

À toda minha família, que estavam sempre ao meu lado me apoiando. Em especial para minha mãe, Ana, que além dos evidentes suportes como mãe, foi uma co-orientadora deste projeto.

À minha namorada, Simone, e sua família, por tudo.

Aos amigos, que estão sempre acompanhando os meus passos.

À Juliana Dias, pela atenção e as indicações sobre a modelagem meteorológica.

A special thanks to professor Carlo Cattani, and his PhD student Aleksey, that found time to discuss and help me to understand the fundamentals of harmonic wavelets. 
"O impossivel existe até que alguém duvide dele e prove o contrário."

Albert Einstein (1879 - 1955) 


\section{Resumo}

Métodos de resolução numérica de equações diferenciais parciais que utilizam ondaletas como base vêm sendo desenvolvidos nas últimas décadas, mas existe uma carência de estudos mais profundos das características computacionais dos mesmos. Neste estudo analisou-se detalhadamente um método espectral de Galerkin com base de ondaletas harmônicas. Revisou-se a teoria matemática referente às ondaletas harmônicas, que mostrou ter grande similaridade com a teoria referente à base trigonométrica de Fourier. Diversos testes numéricos foram realizados. Ao analisarmos a resolução da equação do transporte linear, e também de transporte não linear (equação de Burgers), obtivemos boas aproximações da solução esperada. O custo computacional obtido foi similar ao método com base de Fourier, mas com ondaletas harmônicas foi possível usar a localidade das ondaletas para detectar características de localidade do sinal. Analisamos ainda uma abordagem pseudo-espectral para os casos não lineares, que resultaram em um expressivo aumento de eficiência. Tendo em vista o uso das propriedades de localidade das ondaletas, usamos o método de Galerkin com base de ondaletas harmônicas para resolver um sistema de equações referente a um modelo de propagação de frentes de precipitação. O método mostrou boas aproximações das soluções esperadas, custo computacional ótimo e ainda a possibilidade de se obter espectralmente informações sobre a localização da frente de precipitação.

Palavras-chave: Método de Galerkin, método espectral, método pseudo-espectral, ondaletas, ondaletas harmônicas, modelo de propagação de frentes de precipitação. 


\section{Abstract}

Numerical methods to solve partial differential equations based on wavelets have been developed in the last two decades, but there is a lack of studies on their computational characteristics. In this study a Galerkin spectral method using harmonic wavelets base has been thoroughly analyzed. We performed a review on the mathematics of harmonic wavelets, that showed a great similarity with Fourier basis. Several numerical experiments were made. Analyzing the use of the Galerkin method, with harmonic wavelets, on linear and non linear transport equations, we achieved good approximations in respect to the expected solution. The computational cost resulted to be similar to the same method with Fourier basis. On the other hand, employing harmonic wavelets we were able to obtain local information of the solution by simple inspection of the spectral coefficients. We also analyzed a pseudo-spectral method based on harmonic wavelets for the non linear equations, resulting in a great improvement in efficiency. Looking towards using the locality propriety of harmonic wavelets, we tested the Galerkin method on a precipitation front propagation model. The method resulted in good approximations to the expected solution, optimal computational cost and the possibility of obtaining information on the locality of the precipitation fronts spectrally.

Keywords: Galerkin-Wavelet method, spectral method, pseudo-spectral method, wavelets, harmonic wavelets, precipitation front propagation model. 


\section{Sumário}

$\begin{array}{ll}\text { Lista de Figuras } & \text { xv }\end{array}$

\begin{tabular}{ll}
\hline Lista de Tabelas & xvii
\end{tabular}

\begin{tabular}{ll}
\hline Introdução & 1
\end{tabular}

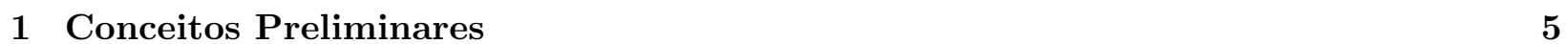

1.1 Normas e Produtos Internos . . . . . . . . . . . . . . . . . . . . . . . . . . 5

1.2 Transformadas de Fourier $\ldots \ldots \ldots \ldots$. . . . . . . . . . . . . 6

$1.2 .1 \quad$ Transformada de Fourier em $L^{2}(\mathbb{R}) \ldots \ldots \ldots \ldots \ldots$

$1.2 .2 \quad$ Transformada de Fourier em $\left.L^{2}([0,1])\right] \ldots \ldots \ldots \ldots$. . . . . . . . . 7

1.2 .3 Transformada Discreta de Fourier . . . . . . . . . . . . . . . . . . . . 9

1.3 Outros conceitos relevantes . . . . . . . . . . . . . . . . . . . . 12

1.3 .1 Base de Riesz . . . . . . . . . . . . . . . . . . . . . 12

1.3 .2 Soma Direta $(\oplus) \ldots \ldots \ldots \ldots \ldots \ldots \ldots$

1.3 .3 Função Indicadora $\ldots \ldots \ldots \ldots \ldots$. . . . . . . . . . . . . . 13

$\begin{array}{lll}2 & \text { Ondaletas } & 15\end{array}$

2.1 Fundamentos . . . . . . . . . . . . . . . . . . . . . . . . . . 15

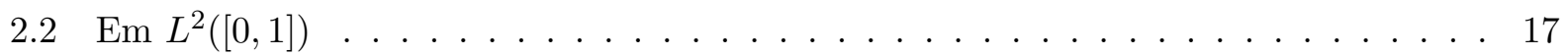

2.3 Exemplos . . . . . . . . . . . . . . . . . . . . . . . . . 19

2.3 .1 Haar . . . . . . . . . . . . . . . . . . . . . . . 19

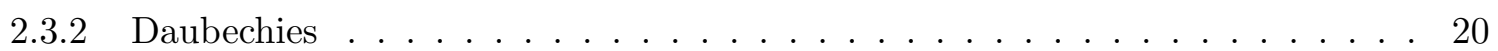

2.3 .3 Meyer . . . . . . . . . . . . . . . . . . . . . . 21

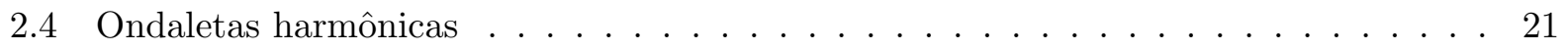

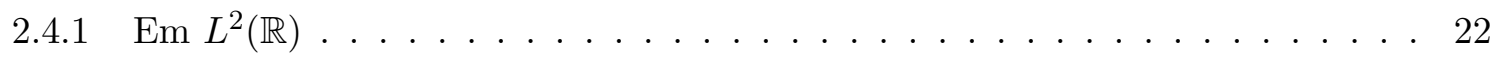

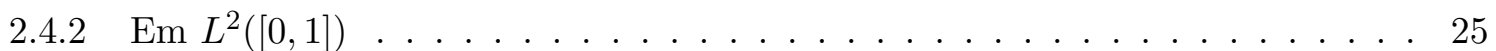

\begin{tabular}{|lll}
\hline 3 & Método de Galerkin & 37
\end{tabular}

3.1 Fundamentos . . . . . . . . . . . . . . . . . . . . . . . 37

3.2 Base de Fourier . . . . . . . . . . . . . . . . . . . . . . . . . 38

$3.2 .1 \quad$ Projeção de derivadas $\ldots \ldots \ldots \ldots \ldots$

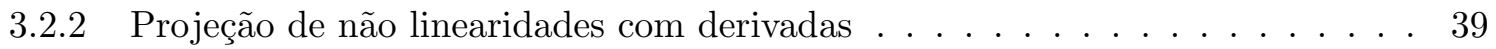


3.2 .3 Problema Teste $\ldots \ldots \ldots \ldots \ldots \ldots \ldots$. . . . . . . . . . . . . . . . 41

3.3 Base de ondaletas harmônicas . . . . . . . . . . . . . . . . . . . . . . . . . 42

3.3 .1 Projeção de derivadas primeiras . . . . . . . . . . . . . . . . . . . 43

3.3 .2 Projeção de derivadas segundas . . . . . . . . . . . . . . . . . . 46

$3.3 .3 \quad$ Projeção de não linearidades com derivadas . . . . . . . . . . . . . . . . 48

3.3 .4 Problema teste . . . . . . . . . . . . . . . . . . . . . 52

$\begin{array}{lll}4 & \text { Testes Numéricos } & 59\end{array}$

4.1 Advecção Linear . . . . . . . . . . . . . . . . . . . . . . . . . . . . . 60

4.2 Advecção-Difusão Não Linear $\ldots \ldots \ldots$. . . . . . . . . . . . . . . . . . 69

4.2 .1 Comparativo com Muniandy e Moroz (1997) . . . . . . . . . . . . . 70

$4.2 .2 \quad$ Custo computacional . . . . . . . . . . . . . . . . . 76

4.2 .3 Métodos de suavização . . . . . . . . . . . . . . . . . . . . . . . . . . . . . . . . . .

$4.2 .4 \quad$ Localidade do sinal . . . . . . . . . . . . . . . . . . . . . . . . . . 85

$4.2 .5 \quad$ Abordagem Pseudo-Espectral . . . . . . . . . . . . . . . . . . 88

\begin{tabular}{|lll}
5 & Aplicações em Meteorologia & 95
\end{tabular}

5.1 Modelo de propagação de frentes de precipitação $\ldots \ldots$. . . . . . . . . . . . . 95

5.2 Experimentos numéricos . . . . . . . . . . . . . . . . 100

\begin{tabular}{lll}
\hline & Conclusões & 113
\end{tabular}

\begin{tabular}{|l|l|}
\hline A Cálculos de Projeções Não Lineares & 115
\end{tabular}

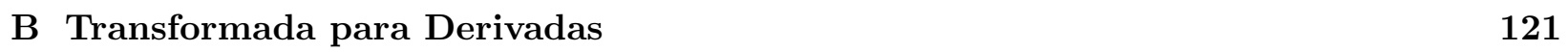

\begin{tabular}{|r|r|}
\hline Referências Bibliográficas & 123
\end{tabular} 


\section{Lista de Figuras}

2.1 Ondaletas de Haar: Pai $(\phi)$ e Mãe $(\psi) \ldots \ldots \ldots \ldots$

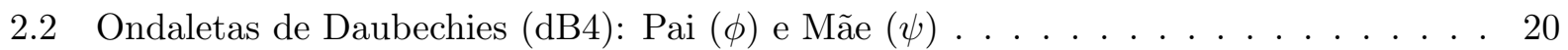

2.3 Ondaletas de Meyer: Pai $(\phi)$ e Mãe $(\psi) \ldots \ldots \ldots \ldots$. . . . . . . . . . 21

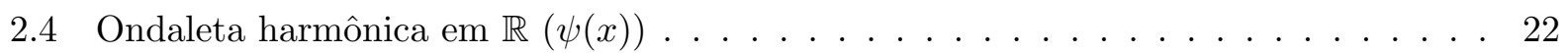

2.5 Ondaleta harmônica pai em $\mathbb{R}(\phi(x)) \ldots \ldots \ldots \ldots \ldots \ldots \ldots$

$2.6 \quad$ Ondaletas harmônicas $\psi_{j k}$ para o intervalo $[0,1] \ldots \ldots \ldots \ldots \ldots \ldots$

2.7 Transformada discreta de Fourier (DFT) das ondaletas harmônicas $\psi_{j k}$ para o inter-

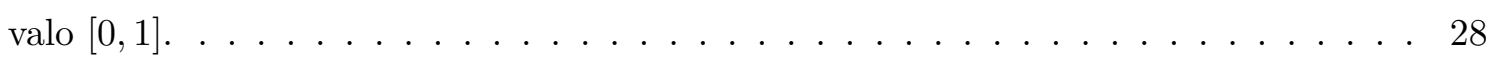

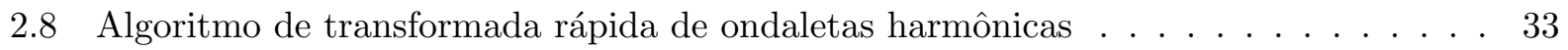

2.9 Teste para transformada de ondaleta harmônica com $n=6$. . . . . . . . . . . . 34

2.10 Teste para transformada de ondaleta harmônica com $n=7$. . . . . . . . . . . 35

2.11 Teste para transformada de ondaleta harmônica com $\mathrm{n}=8$. . . . . . . . . . . 36

$3.1 \quad$ Valores absolutos dos coeficientes de conexão não lineares $P(0)$. . . . . . . . . . . . 50

3.2 Logaritmo dos valores absolutos dos coeficientes de conexão não lineares $P(0)$. . . . 51

3.3 Valores absolutos dos coeficientes de conexão não lineares $N_{k q}^{j p} \ldots \ldots$. . . . . . . . 52

3.4 Logaritmo dos valores absolutos dos coeficiente de conexão não lineares $P(0)$ usando localidade do suporte da base . . . . . . . . . . . . . . . . . 53

4.1 Advecção de função exponencial com ondaletas harmônicas . . . . . . . . . . . . . . 62

4.2 Evolução do erro e coeficientes na advecção de função exponencial com ondaletas

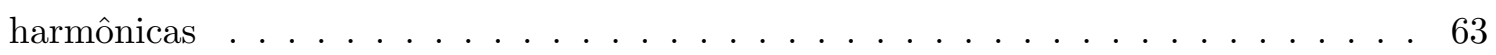

4.3 Advecção de função exponencial com ondaletas harmônicas com corte no suporte

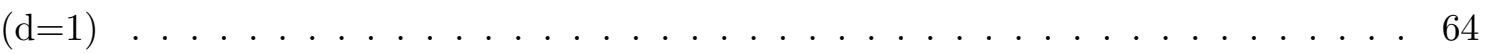

4.4 Advecção de função exponencial com ondaletas harmônicas com corte no suporte

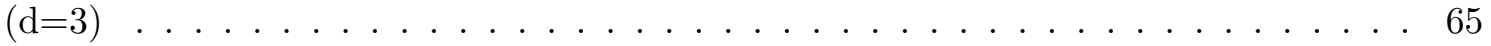

4.5 Adveç̧ão dos coeficientes de ondaletas harmônicas da função exponencial . . . . . . 66

4.6 Solução numérica da equação de Burgers obtida em Muniandy e Moroz [1997)] . . . 71

4.7 Solução numérica da equação de Burgers para condição inicial senoidal . . . . . . . . 73

4.8 Solução numérica da equação de Burgers para condição inicial senoidal . . . . . . . . 74

4.9 Solução numérica da equação de Burgers para condição inicial senoidal . . . . . . . . 75 
4.10 Solução numérica da equação de Burgers para condição inicial exponencial usando simplificação por frequências vizinhas . . . . . . . . . . . . . . . . 76

4.11 Solução numérica da equação de Burgers para condição inicial senoidal com aplicação de métodos de suavização . . . . . . . . . . . . . . . . . . . . . . . . 81

4.12 Solução numérica da equação de Burgers para condição inicial exponencial com aplicação de métodos de suavização . . . . . . . . . . . . . . . . . . . . . . . . 82

4.13 Solução numérica da equação de Burgers para condição inicial exponencial com aplicação de métodos de suavização . . . . . . . . . . . . . . . . . . . 83

4.14 Solução numérica da equação de Burgers para condição inicial exponencial com aplicação de métodos de suavização . . . . . . . . . . . . . . . . . . . . . 84

4.15 Solução numérica da equação de Burgers para condição inicial de pulso . . . . . . . . 86

4.16 Evolução da solução numérica da equação de Burgers para condição inicial exponencial 87

4.17 Método pseudo-espectral para a equação de Burgers com condição inicial exponencial 89

4.18 Método pseudo-espectral para a equação de Burgers com condição inicial exponencial 90

4.19 Método pseudo-espectral para a equação de Burgers com condição inicial senoidal. . 91

4.20 Solução para a equação de Burgers obtida de Schult e Wyld [1992)] . . . . . . . . . . 92

4.21 Método pseudo-espectral para a equação de Burgers com condição inicial senoidal truncada . . . . . . . . . . . . . . . . . . . . . . . 93

5.1 Diagrama para frente atenuante $\ldots \ldots \ldots$. . . . . . . . . . . . 100

5.2 Condições iniciais para frente atenuante $\ldots \ldots \ldots$. . . . . . . . . . . . 101

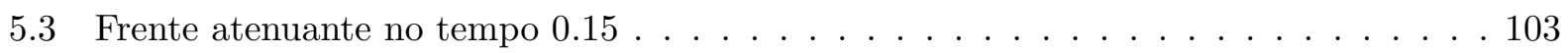

5.4 Frente atenuante no tempo 0.3 . . . . . . . . . . . . . . . . . . 104

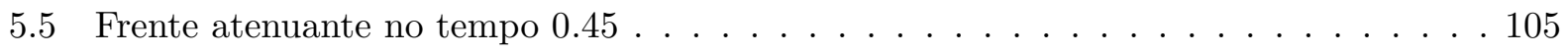

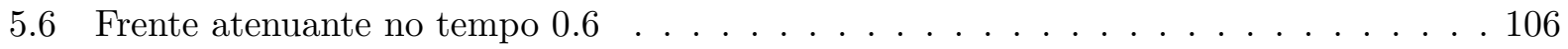

$5.7 \quad$ Frente atenuante no tempo $0.9 \quad \ldots \ldots \ldots \ldots$

5.8 Evolução da frente atenuante $\ldots \ldots \ldots$. . . . . . . . . . . . . . . . . . 108

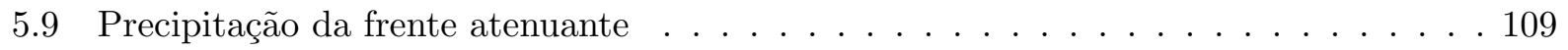

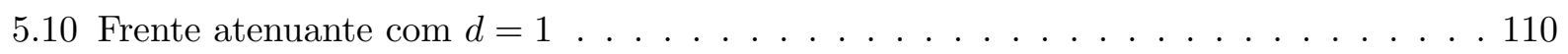

5.11 Frente atenuante com $d=3 \ldots \ldots \ldots \ldots \ldots$

5.12 Detalhes da frente atenuante com $d=3 \ldots \ldots \ldots$. . . . . . . . . . . . . . . . 


\section{Lista de Tabelas}

2.1 Percentual do suporte de ondaletas harmônicas $\psi_{j, k}(x)$ restrito ao intervalo $I_{j k}^{d}$. . . 30

2.2 Percentual do suporte da derivada primeira de ondaletas harmônicas $\frac{d \psi_{j, k}(x)}{d x}$ restrito

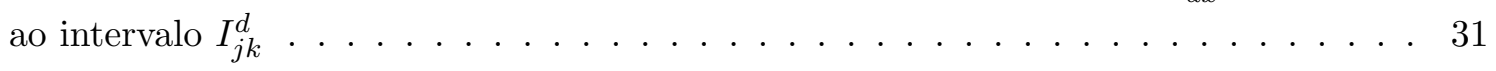

2.3 Erros associados à transformada de ondaletas harmônicas . . . . . . . . . . . . . . 35

4.1 Tempo de execução e erro para resolução da equação do transporte por ondaletas

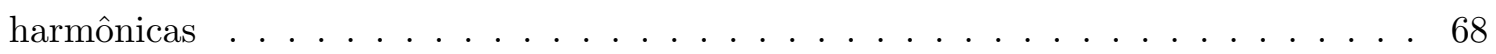

4.2 Tempo de execução do algoritmo para resolução da equação de Burgers por ondaletas

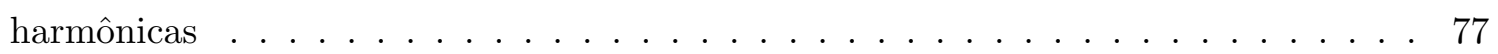

4.3 Tempo de execução do algoritmo para resolução da equação de Burgers por Fourier. 78

4.4 Tempo de execução em relação ao erro do algoritmo de ondaletas harmônicas para resolução da equação de Burgers em relação . . . . . . . . . . . . . . . . . . . . . . . 79 
xviii 


\section{Introdução}

\section{Métodos espectrais}

A área de interesse predominante deste estudo é a da utilização de ondaletas, em particular as ondaletas harmônicas, em métodos para a resolução de equações diferenciais parciais (EDPs). Temos como motivação o propósito de aplicar estes métodos na resolução de problemas ligados à fenômenos meteorológicos. Apesar do interesse em resolver problemas da meteorologia, a abordagem é bastante geral para ser utilizada em outras áreas. Muitos são os métodos para resolução de EDPs, como, por exemplo, diferenças finitas, elementos finitos, volumes finitos, entre outros. Nos concentraremos em métodos espectrais, por uma motivação que ficará mais clara adiante. Seu desenvolvimento se deu principalmente de 1970 até os dias atuais. Antes da década de 70 os métodos espectrais eram tidos como computacionalmente caros, principalmente para uso em previsão do tempo, e foi somente com o desenvolvimento do método das transformadas por Orzag (1972), e simultaneamente por Eliasen, Machenhauer e Rasmussen (1970), que o estudo sobre métodos espectrais avançou e ganhou competitividade.

Neste trabalho vamos nos voltar para o método espectral conhecido por Método de Galerkin. Trata-se de um método onde tenta-se resolver o problema escrevendo a solução em uma base de interesse, desta forma é possível transformar problemas com operadores contínuos, como problemas de equações diferenciais, em problemas discretos. Uma abordagem interessante é a do método de Galerkin com base de Fourier, onde em muitos casos é possível transformar o problema do espaço para o domínio de frequências, resolver o problema no domínio de frequências, e retornar ao domínio do espaço. Esta abordagem tem a grande vantagem de ser toda fundamentada na teoria de séries de Fourier e transformadas de Fourier, cujos conceitos já são amplamente conhecidos e estudados, com algoritmos numéricos muito eficientes, porém é preferencialmente indicado para problemas periódicos (PEYRET, 2000).

Detalharemos nesta dissertação principalmente o Método de Galerkin, mas deixamos aqui indicações gerais para outros métodos espectrais. A metodologia envolvida nos métodos espectrais está bem explicada em diversos livros e artigos, indicamos aqui aqueles mais voltados à resolução de problemas ligados à dinâmica de fluidos. O livro de Peyret (2000) é indicado para estudos em fluidos incompressíveis viscosos, com um enfoque grande em métodos espectrais de Fourier e Chebyshev. O livro de Boyd (1999) mostra um enfoque maior em métodos espectrais de Chebyshev. Em Canuto et al. (1988) são abordados métodos espectrais para fluidos em geral, mas motivado a resolver problemas da meteorologia. Em Gottlieb e Orszag (1977), Voigt (1984) e Machenhauer 
(1979), pode-se encontrar detalhes da metodologia espectral para modelos meteorológicos.

Vale ressaltar que hoje em dia diversos centros de previsão do tempo utilizam-se de métodos espectrais, incluindo o Centro de Previsão do Tempo e Estudos Climáticos (CPTEC), no Brasil, mostrando sua competitividade em relação à outros métodos. Porém, o que ocorre hoje, é que é comum encontrarmos modelos espectrais em que se usam polinômios de Legendre, na forma de Harmônicos Esféricos, para os quais não há transformada rápida, como no caso de Fourier, com isso o método das transformadas fica com um maior custo computacional. Explorando diversas propriedades dos polinômios de Legendre o método fica competitivo, porém é um ponto fraco. Mas fica claro que é uma área de estudo que merece atenção. Em particular estamos interessados em olhar para outras bases de funções que possam servir em um método espectral que seja eficiente, ou pelo menos tenha propriedades interessantes que façam valer seu uso.

\section{Ondaletas e os métodos espectrais}

Em paralelo com o desenvolvimento dos métodos espectrais para EDPs, nas últimas décadas muitos pesquisadores têm explorado o uso de ondaletas (tradução para o português de wavelets (MORETTIN, 1999)) na resolução de problemas de diversas áreas do conhecimento, tendo uma expressão mais acentuada no processamento de imagens e sinais. Trata-se de uma metodologia de decomposição de uma função, ou sinal, em um domínio de frequências e espaço, conhecida hoje por análise multi-resolução. Desta forma, com o uso de ondaletas, é possível investigar a ocorrência de fenômenos localizados no espaço e frequência simultaneamente. Nas últimas duas décadas pesquisadores têm buscado utilizar essa e outras características de ondaletas para resolver problemas de equações diferenciais. A idéia é explorar as propriedades de ondaletas, principalmente o fato de conseguir representar estruturas complexas, com singularidades por exemplo, com baixos graus de liberdade, para resolver problemas de EDP em que de fato aparecem singularidades, estruturas complexas, frentes localizadas entre outros problemas BENEDETTO; FRAZIER, 1994. VASILYEV; YUEN; PAOLUCCI, 1997).

Um trabalho que detalhou bem o uso de ondaletas para problemas de equações diferenciais em dinâmica de fluidos é o de Jaffard e Laurençot (1992), onde discutem-se diversos pontos de vista em relação ao assunto. Uma das possibilidades a serem exploradas é de se considerar métodos adaptativos (BACRY; MALLAT; PAPANICOLAOU, 1992; LIU, 2003; MEHRA; KEVLAHAN, 2008; DOMINGUES et al., 2008; VASILYEV; KEVLAHAN, 2005; BEYLKIN; KEISER, 1997; CAI; WANG, 1996; ALPERT et al., 2002; GOEDECKER; IVANOV, 1998; KOZAKEVICIUS, 2001; DOMINGUES, 2001), que usam ondaletas para, por exemplo, definir regiões de refinamento de malha, ou com adaptatividade no tempo. Os primeiros estudos neste sentido apareceram no final da década de 80, começo da década de 90, na França, por Maday, Perrier e Ravel (1991), mas logo diversos outros estudos apareceram. Existem diversas formas de se considerar bases de ondaletas na resolução de EDPs, a adaptatividade de malha é apenas uma delas.

Outra abordagem interessante é a de se usar ondaletas para pre-condicionar um operador diferencial, reduzindo seu número de condição. Destacamos aqui o trabalho que vem sendo desenvolvido com este enfoque por Bertoluzza e Naldi (1996), propondo um método de colocação 
(pseudo-espectral) fundamentado em pre-condicionadores de ondaletas. Um pouco do que será desenvolvido neste trabalho teve como base as notas de aula de Bertoluzza (2007) para a Escola Avançada de Simulação e Análise Numérica de EDPs, realizada em Barcelona em 2007.

Nosso estudo porém seguirá a linha de se utilizar ondaletas como base para um método de Galerkin, o que ficou conhecido como método de Galerkin-Ondaleta (traduzida do inglês GalerkinWavelet) GOMES; CORTINA, 1996; NAIR, 2004; FRAZIER, 1999), e resolver o problema no domínio de ondaletas. Para tanto surge uma primeira indefinição: Qual base de ondaletas usar? Existem diversas possibilidades, nota-se um grande uso das ondaletas de Daubechies para este propósito BESORA, 2004, HO; YANG, 2001, SCHULT; WYLD, 1992, AMARATUNGA et al., 1994) , mas também de outras bases, como ondaletas de Meyer (LOPES; MATTOS, 2003), ondaletas de Haar (LEPIK, 2007) e Coiflets (LIN; ZHOU, 2001). Boa parte destes trabalhos está voltado para resolver problemas de EDPs não lineares em uma dimensão espacial, e é comum usarem como teste a equação de Burgers (JAFFARD; LAURENÇOT, 1992).

Dos trabalhos que analisam métodos fundamentados em ondaletas para resolver problemas de EDPs, conclui-se que tais métodos geralmente resultam em boas aproximações para a solução desejada. Muitos afirmam que os métodos são precisos e eficientes, e portanto tem perspectivas de se tornarem numericamente viáveis na resolução de problemas de EDPs. Analisam a convergência e o erro gerado pela aproximação numérica, porém pouco se fala sobre o custo computacional envolvido na resolução via ondaletas em relação à métodos clássicos de resolução de EDPs. No trabalho de Besora (2004) foram analisados os números de condição das matrizes de conexão em problemas lineares, como o da equação da onda, e os resultados mostram números de condição altos, o que pode aumentar diretamente o custo computacional da resolução do sistema linear a ser resolvido. Do trabalho de Schult e Wyld (1992) conclui-se que as ondaletas de Daubechies resolvem bem a equação de Burgers, mas com custo computacional alto quando comparado a diferenças finitas, assim como em Lazaar et al. (1994), que mostra ordem $N^{4}$ de custo computacional do método com ondaletas "splines" periódicas. O trabalho de Bacry, Mallat e Papanicolaou (1992) refere-se brevemente à possível alta complexidade computacional para casos não lineares. Temos interesse, nesta dissertação, de olhar mais a fundo questões ligadas à complexidade computacional em métodos fundamentados em ondaletas para resolução de EDPs, a fim de verificar de fato a viabilidade prática dos mesmos.

Usaremos como base para este estudo as ondaletas harmônicas, ou ondaletas de Newland. Desenvolvidas por Newland (1993), a base de ondaletas harmônicas possui, além das propriedades de análise de multi-resolução, outras características favoráveis ao estudo de EDPs, como diferenciabilidade infinita, suporte compacto no domínio de frequências, algoritmo de transformada rápido e muita similaridade com bases de Fourier, o que faz com que possamos aproveitar propriedades boas de Fourier nas ondaletas harmônicas. Um primeiro trabalho que usou ondaletas harmônicas na resolução de EDPs, em particular para resolver a equação de burgers, foi o de Muniandy e Moroz (1997), e é com base neste trabalho que nosso estudo foi desenvolvido. No trabalho de Muniandy e Moroz (1997) destacam-se as possibilidades de se aproveitar características da base de ondaletas 
harmônicas para a obtenção de um método com baixo custo computacional, mas o trabalho não analisa a fundo questões computacionais. O estudo do elo entre ondaletas harmônicas e EDPs foi aprofundado por Cattani (CATTANI, 2003, CATTANI, 2005; CATTANI, 2008), porém sua abordagem é mais analítica e esta dissertação tem enfoque mais numérico.

Com interesse em analisar numericamente um método de resolução de EDPs, com possíveis não linearidades, optamos por usar o método de Galerkin-Ondaleta com uma base de ondaletas harmônicas. O trabalho mais próximo desta abordagem é o de Muniandy e Moroz (1997), conforme dito antes, porém concentramo-nos no presente trabalho a aprofundar tanto questões de construção da base de ondaletas harmônicas para intervalos limitados, tendo em vista que o método terá que ser implementado numericamente, quanto à questões de custo computacional e eficiência numérica. Além disso algumas generalizações foram propostas e nos desafiamos a testar o método em equações ligados à fenômenos meteorológicos.

\section{Aplicação em meteorologia}

Descrevemos um modelo de propagação de frentes de precipitação unidimensional, proposto por Frierson, Majda e Pauluis (2004), para a realização de testes com o método de Galerkin com base de ondaletas harmônicas. Este modelo constitui uma boa aproximação de fenômenos convectivos ao redor do equador. Apesar do modelo ser linear, este pode apresentar frentes de propagação no campo de precipitação, e por isso pode ser interessante o uso de ondaletas na sua resolução.

\section{Delineamento do trabalho}

Após um breve capítulo de preliminares matemáticas, necessárias para a compreensão dos demais capítulos, discutimos no capítulo 2 características relevantes em relação às ondaletas, e analisamos mais a fundo o caso particular das ondaletas harmônicas. No capítulo 3 deduzimos as expressões para a resolução de alguns exemplos de EDPs, lineares e não lineares, com o método de Galrkin utilizando as bases de Fourier e ondaletas harmônicas. Com estes 3 capítulos esperamos ter dado subsíduo teórico suficiente para justificar o capítulo 4, onde realizamos uma série de testes numéricos com o método de Galerkin usando base de ondaletas harmônicas. São analisadas questões ligadas à precisão e eficiência para a equação do transporte linear e não linear (Burgers). No capítulo 5 testamos o método de Galerkin com ondaletas harmônicas no modelo de propagação de frente de precipitação proposto por Frierson, Majda e Pauluis (2004). Encerramos o trabalho com as conclusões obtidas, no capítulo 6 . 


\section{Capítulo 1}

\section{Conceitos Preliminares}

Vamos apresentar inicialmente algumas convenções de notação, definições e teoremas relevantes para o decorrer do texto.

\subsection{Normas e Produtos Internos}

Definiremos a seguir as normas e produtos internos que serão usados ao longo do trabalho.

Definição 1.1.1. Definimos como produto interno para o espaço de Hilbert das funções mensuráveis, reais ou complexas, quadrado integráveis em $\mathbb{R}$, ou em um intervalo $I \subseteq \mathbb{R}$, com notações usuais $L^{2}(\mathbb{R})$, ou $L^{2}(I)$, o seguinte:

$$
\langle f, g\rangle_{L^{2}(I)}=\int_{I} f g^{*}
$$

onde $g^{*}$ representa o complexo conjugado de g. E a norma proveniente deste produto interno:

$$
\|f\|_{L^{2}(I)}^{2}=\langle f, f\rangle_{L^{2}(I)}
$$

Definição 1.1.2. Definimos como produto interno para o espaço de Hilbert $l^{2}(\mathbb{Z})$, das sequências quadrado somáveis $\left\{f_{0}, f_{1}, \ldots, f_{n}, \ldots\right\}$, reais ou complexas, o seguinte:

$$
\langle f, g\rangle_{l^{2}(\mathbb{Z})}=\sum_{j=0}^{\infty} f_{j} g_{j}^{*}
$$

onde $g_{j}^{*}$ representa o complexo conjugado de $g_{j}$. E a norma resultante desde produto interno:

$$
\|f\|_{l^{2}(\mathbb{Z})}^{2}=\langle f, f\rangle_{l^{2}(\mathbb{Z})}
$$

Definição 1.1.3. Definimos como produto interno para o espaço de dimensão finita dos valores de uma função $f$, real $\left(\mathbb{R}^{n}\right)$ ou imaginária $\left(\mathbb{C}^{n}\right),\left\{f_{1}, f_{2}, \ldots, f_{N}\right\}$, definida nos pontos $\left\{x_{1}, x_{2}, \ldots, x_{N}\right\}$, como:

$$
\langle f, g\rangle_{N}=\frac{1}{N} \sum_{j=1}^{N} f_{j} g_{j}^{*}
$$


onde $g_{j}^{*}$ representa o complexo conjugado de $g_{j}$. E com norma:

$$
\|f\|_{N}^{2}=\langle f, f\rangle_{N}
$$

Definição 1.1.4. Diremos que uma função $f$ é regular quando esta for $\mathcal{C}^{1}$ em $\mathbb{R} e \exists C>0, \forall x \in \mathbb{R}$, satisfizer as propriedades

$$
|f(x)|,\left|f^{\prime}(x)\right| \leq \frac{C}{1+x^{2}} .
$$

Definição 1.1.5. Definimos como norma de uma matriz $A$, com dimensões $n \times n$, o seguinte,

$$
\|A\|:=\sup \left\{\frac{\|A x\|}{\|x\|}: x \in \mathbb{R}^{n}, x \neq 0\right\}
$$

onde $\|\cdot\|=\|\cdot\|_{N}$ é a norma definida em 1.1.3.

Definição 1.1.6. Denominamos por número de condição de uma matriz $A$, com dimensões $n \times n$, invertível, o seguinte,

$$
\kappa_{A}:=\|A\|\left\|A^{-1}\right\|
$$

Denominamos por matriz esparsa uma matriz que possua "a maioria das entradas" nulas. Ela é esparsa e estruturada quando os elementos não nulos, em minoria, seguem algum padrão, ou fórmula, para determinação de suas posições.

\subsection{Transformadas de Fourier}

Destacaremos aqui algumas definições e propriedades referentes às transformadas de Fourier contínua e discreta. Uma referência mais avançada para esta parte é o livro de Rudin (1987), onde é possível verificar as demonstrações e mais detalhes das propriedades da transformada de Fourier. Para características mais básicas consulte o livro de Chui (1992).

\subsubsection{Transformada de Fourier em $L^{2}(\mathbb{R})$}

Definição 1.2.1. Seja $f \in L^{2}(\mathbb{R})$, então denotamos por sua transformada contínua de Fourier direta a função dada por

$$
\hat{f}(\omega)=(\mathcal{F} f)(\omega)=\frac{1}{2 \pi} \int_{\mathbb{R}} f(x) e^{-i \omega x} d x
$$

$\operatorname{com} \omega \in \mathbb{R}$.

Definição 1.2.2. Seja $f \in L^{2}(\mathbb{R})$, então denotamos por sua transformada contínua de Fourier inversa a função dada por

$$
\check{f}(x)=\left(\mathcal{F}^{-1} f\right)(x)=\int_{\mathbb{R}} \hat{f}(\omega) e^{i \omega x} d \omega
$$

$\operatorname{com} x \in \mathbb{R}$.

A transformada de Fourier admite algumas propriedades, que usaremos mais adiante, e portanto destacaremos aqui.

Proposição 1.2.3. Propriedades da transformada de Fourier: 
(a) Translação e dilatação. Sejam $a, b \in \mathbb{R}$, com $a \neq 0$, então

$$
(f(\widehat{a x+b}))(\omega)=\frac{1}{a} e^{i \omega b / a} \hat{f}(\omega / a)
$$

(b) Teorema de Plancherel

$$
\begin{gathered}
\int_{-\infty}^{\infty} f(x) g(x)^{*} d x=2 \pi \int_{-\infty}^{\infty} \hat{f}(\xi) \hat{g}(\xi)^{*} d \xi \\
\langle f, g\rangle_{L^{2}(\mathbb{R})}=2 \pi\langle\hat{f}, \hat{g}\rangle_{L^{2}(\mathbb{R})}
\end{gathered}
$$

(c) Fórmula de Parseval

$$
\|f\|_{L^{2}(\mathbb{R})}=\sqrt{2 \pi}\|\hat{f}\|_{L^{2}(\mathbb{R})}
$$

(d) Inversa de Fourier de uma transformada de Fourier

$$
f(x)=\left(\mathcal{F}^{-1} \hat{f}\right)(x)
$$

Demonstração. As demonstrações utilizam outros conceitos que não destacaremos aqui, mas podem ser encontrados em Chui (1992), ou em diversas outras referências sobre Transformadas de Fourier.

\subsubsection{Transformada de Fourier em $L^{2}([0,1])$}

Consideramos o espaço das funções $f$, quadrado integráveis no intervalo unitário, periódicas em $[0,1]$, com período 1 , no sentido de que $f(0)=f(1)$. Neste espaço adotaremos a transformada de Fourier da seguinte forma:

Definição 1.2.4. Seja $f \in L^{2}([0,1])$, então denotamos por sua transformada contínua de Fourier direta a função dada por

$$
\hat{f}_{\omega}=(\mathcal{F} f)(\omega)=\int_{0}^{1} f(x) e^{-2 \pi i \omega x} d x
$$

$\operatorname{com} \omega \in \mathbb{Z}$.

Destacaremos alguns teoremas importantes que serão usados em análises que aparecem mais adiante no trabalho. Não vamos demonstrar estes teoremas por se tratarem de resultados comumente encontrados em livros de análise funcional, como Rudin (1987) ou Saxe (2000).

Teorema 1.2.5 (Série de Fourier). Seja $f=\sum_{k=1}^{\infty} c_{k} f_{k}$, com $\left\{f_{k}\right\}_{k=1,2, \ldots}$ uma sequência ortonormal em um espaço com produto interno $V$. Então $c_{k}=\left\langle f, f_{k}\right\rangle_{V}$ para cada $k$.

$A$ série $f=\sum_{k=1}^{\infty} c_{k} f_{k}$ é denominada Série de Fourier de $f$ em relação à $\left\{f_{k}\right\}_{k=1,2, \ldots}, e\left\langle f, f_{k}\right\rangle_{V}$ de Coeficientes de Fourier. 
No caso de termos polinômios trigonométricos, $f_{k}=e^{2 \pi i k x}$, com $k \in \mathbb{Z}$, e $V=L^{2}([0,1])$, então teremos os coeficientes dados por

$$
c_{k}=\left\langle f, f_{k}\right\rangle_{L^{2}([0,1])}=\int_{0}^{1} f(x) e^{-2 \pi i k x} d x=\hat{f}_{k} .
$$

e a série de Fourier, que pode ser vista como inversa da transformada de Fourier para o $L^{2}([0,1])$, dada por

$$
f=\sum_{k=-\infty}^{\infty} \hat{f}_{k} e^{2 \pi i k x} .
$$

Teorema 1.2.6 (Convergência de Série de Fourier). Seja u uma função contínua, periódica em $[0,1]$, e período 1, assim como suas derivadas $u^{(l)}$ até grau $m-1$, e com m-ésima derivada módulo integravel. Considere a expansão de u em sua série de fourier truncada:

$$
u_{K}=\sum_{k=-K}^{K} \hat{u}_{k} e^{2 \pi i k x} .
$$

Então teremos que

$$
\hat{u}_{k}=O\left(|k|^{-m}\right), \quad \text { para } k \rightarrow \infty .
$$

e

$$
\left\|u-u_{K}\right\|_{L^{2}([0,1])} \leq C K^{-m}\left\|u^{(m)}\right\|_{L^{2}([0,1])}
$$

onde $C>0$ é constante independente de $K>0$.

Demonstração. Indicamos aqui o livro de Peyret (2000) para a demonstração desse interessante teorema, que garante na verdade uma convergência dita exponencial da aproximação por séries de Fourier para funções infinitamente diferenciáveis.

Teorema 1.2.7 (Teorema de Plancherel). Suponha $f, g \in L^{2}([0,1])$ ambas expandidas em suas Séries de Fourier com polinômios trigonométricos. Então vale que

$$
\langle f, g\rangle_{L^{2}([0,1])}=\langle\hat{f}, \hat{g}\rangle_{l^{2}(\mathbb{Z})} .
$$

Demonstração. Escrevendo $f$ e $g$ como séries trigonométricas

$$
\begin{aligned}
& f=\sum_{k=-\infty}^{\infty} \hat{f}_{k} e^{2 \pi i k x}, \\
& g=\sum_{w=-\infty}^{\infty} \hat{g}_{w} e^{2 \pi i w x},
\end{aligned}
$$


usando o fato de que as séries convergem e o teorema da convergência dominada, temos que

$$
\begin{aligned}
\langle f, g\rangle_{L^{2}([0,1])} & =\int_{0}^{1} \sum_{k=-\infty}^{\infty} \sum_{w=-\infty}^{\infty} \hat{f}_{k} e^{2 \pi i k x} \hat{g}_{w}^{*} e^{-2 \pi i w x} d x \\
& =\sum_{k=-\infty}^{\infty} \sum_{w=-\infty}^{\infty} \hat{f}_{k} \hat{g}_{w}^{*} \int_{0}^{1} e^{2 \pi i(k-w) x} d x \\
& =\sum_{k=-\infty}^{\infty} \sum_{w=-\infty}^{\infty} \hat{f}_{k} \hat{g}_{w}^{*} \delta_{w, k} \\
& =\langle\hat{f}, \hat{g}\rangle_{l^{2}(\mathbb{Z})} .
\end{aligned}
$$

Teorema 1.2.8 (Identidade de Parseval). Suponha uma sequência ortogonal $\left\{f_{k}\right\}_{k=1,2, \ldots}$ em um espaço com produto interno $V$. Então $\left\{f_{k}\right\}_{k=1,2, \ldots}$ é uma sequência ortogonal completa, no sentido de que se $f \in V$ é possível encontrarmos constantes $c_{k}$ dependentes de $f$ tais que $f=\sum_{k=1}^{\infty} c_{k} f_{k}$, se, e somente se, para todo $f \in V$,

$$
\sum_{k=1}^{\infty}\left|\left\langle f, f_{k}\right\rangle_{V}\right|^{2}=\|f\|^{2} .
$$

Teorema 1.2.9. Suponha $\left\{d_{k}\right\}_{k=1,2, \ldots}$ uma sequência de números reais em $l^{2}(\mathbb{Z})$ e $V$ um espaço de Hilbert com sequência ortonormal completa $\left\{f_{k}\right\}_{k=1,2, \ldots}$. Então existe um elemento $f \in V$ tal que seus coeficientes de Fourier em relação à $\left\{f_{k}\right\}_{k=1,2, \ldots}$ são os números $\left\{d_{k}\right\}_{k=1,2, \ldots}$ e que

$$
\|f\|_{V}^{2}=\sum_{k=1}^{\infty} d_{k}^{2} .
$$

Teorema 1.2.10. Para uma sequência ortonormal $\left\{f_{k}\right\}_{k=1,2, \ldots} \in L^{2}(I)$ as afirmativas a seguir são equivalentes:

1. $\left\{f_{k}\right\}_{k=1,2, \ldots}$ é sequência ortonormal completa, no sentido de que se $f \in V$ é possível encontrarmos constantes $c_{k}$ dependentes de $f$ tais que $f=\sum_{k=1}^{\infty} c_{k} f_{k}$.

2. $\forall f \in L^{2}$ e $\epsilon>0$ existe uma combinação linear finita $g=\sum_{k=1}^{N} d_{k} f_{k}$ tal que $\|f-g\|_{L^{2}}<\epsilon$

3. Se os coeficientes de Fourier com relação à $\left\{f_{k}\right\}_{k=1,2, \ldots}$ de uma função em $L^{2}$ são nulos, então a função é nula quase sempre.

\subsubsection{Transformada Discreta de Fourier}

Definição 1.2.11. Definimos para o domínio discreto finito, $\mathbb{C}^{N}$ ou $\mathbb{R}^{N}$, como transformada direta de Fourier, considerando pontos $\left\{x_{l}\right\}_{l=0,1, \ldots, N-1}$ em $[0,1]$, uniformemente espaçados $\left(x_{l}=l / N\right)$, e 
$f_{l}=f\left(x_{l}\right)$, números reais ou complexos, o seguinte:

$$
\hat{f}_{w}=\frac{1}{N} \sum_{l=0}^{N-1} f_{l} e^{-2 \pi i w x_{l}}, \quad w=-\frac{N}{2},-\frac{N}{2}+1, . ., \frac{N}{2}-1 .
$$

com inversa dada por

$$
\check{f}_{l}=\sum_{w=-\frac{N}{2}}^{\frac{N}{2}-1} \hat{f}_{w} e^{2 \pi i w x_{l}}, \quad l=0,1, \ldots N-1 .
$$

Aqui vale um comentário interessante, se $f$ for da forma $f=\sum_{k=-N / 2}^{N / 2-1} c_{k} e^{2 \pi i k x}$, então a transformada discreta de Fourier, dada na definição (1.2.11), é equivalente à transformada contínua de Fourier para o $L^{2}([0,1])$, dada na definição 1.2 .4 ). Isso ocorre pois a expressão dada para a transformada discreta é uma fórmula para integração em $[0,1]$ exata para polinômios trigonométricos com grau menor ou igual a $N$ (STOER; BULIRSCH, 1992).

Quando $N$ é da forma $N=2^{n}$ estas transformadas, direta e inversa, podem ser calculadas com algoritmos rápidos (Fast Fourier Transform - FFT), isto é, ao invés de realizar os cálculos com ordem $N^{2}$ operações, obtém-se a transformada com ordem de $N \log _{2}(N)$ operações. Existem generalizações para a forma em que $N$ deve estar, mas para o nosso estudo nos bastará ser potência de 2. O leitor pode encontrar mais detalhes do algoritmo em diversos textos relacionados à Análise Numérica, deixamos como referência o livro de Stoer e Bulirsch (1992).

No domínio discreto finito temos um conjunto importante de propriedades, destacadas a seguir.

Proposição 1.2.12. Propriedades da transformada de Fourier discreta

1. $\operatorname{Para} k=-N / 2, \ldots, N / 2-1$,

$$
\sum_{j=0}^{N-1} e^{ \pm 2 \pi i k j / N}= \begin{cases}0 & k \neq 0 \\ N & k=0\end{cases}
$$

2. $\operatorname{Para} j=0,1, \ldots, N-1$,

$$
\sum_{k=-N / 2}^{N / 2-1} e^{ \pm 2 \pi i k j / N}= \begin{cases}0 & j \neq 0 \\ N & j=0\end{cases}
$$

3. Caso geral, $j \in \mathbb{Z}$

$$
\sum_{k=-N / 2}^{N / 2-1} e^{ \pm 2 \pi i k j / N}= \begin{cases}N & j=m N, \quad m=0, \pm 1, \pm 2, \ldots 0 \\ 0 & \text { caso contrário. }\end{cases}
$$

\section{Periodicidade}

$$
\hat{f}_{w}=(\hat{f})_{w+N} .
$$


5. Se $f(x)$ é real $(f \in \mathbb{R})$,

$$
\hat{f}_{w}=(\hat{f})_{-w}^{*}
$$

Demonstração. Propriedades da transformada discreta de Fourier

1. Se $k=0$, então é trivial. Supondo $0<k<N / 2-1$, vamos mostrar para o caso de expoente positivo, e o negativo segue análogo. E o caso de $-N / 2<k<0$ também segue análogo.

$$
\begin{aligned}
\sum_{j=0}^{N-1} e^{2 \pi i k j / N} & =1+e^{2 \pi i k / N}+e^{4 \pi i k / N}+\ldots+e^{2(N-1) \pi i k / N} \\
& =\frac{e^{(2 \pi i k / N) N}-1}{e^{2 \pi i k / N}-1} \\
& =0 .
\end{aligned}
$$

2. Idem ao ítem anterior.

3. Idem ao ítem anterior.

4. Lembrando que $x_{l}=l / N$ temos que,

$$
(\hat{f})_{w+N}=\frac{1}{N} \sum_{l=0}^{N-1} f_{l} e^{-2 \pi i(w+N) x_{l}}=(\hat{f})_{w} \frac{1}{N} \sum_{l=0}^{N-1} f_{l} e^{-2 \pi i l}=(\hat{f})_{w} .
$$

5. Para $f$ real temos que $f=f^{*}$, então

$$
(\hat{f})_{-w}^{*}=\frac{1}{N} \sum_{l=0}^{N-1}\left(f_{l}\right)^{*}\left(e^{-2 \pi i(-w) x_{l}}\right)^{*}=\left(\hat{f}^{*}\right)_{w}=(\hat{f})_{w} .
$$

Teorema 1.2.13 (Teorema de Plancherel). Para os conjuntos de valores $\left\{f_{l}\right\}_{l=0,1, \ldots, N-1}$ e $\left\{g_{l}\right\}_{l=0,1, \ldots, N-1}$, reais ou complexos, definidos sobre uma malha discreta no intervalo unitário $\left(\left\{x_{l}=l / N\right\}_{l=0,1, \ldots, N-1}\right)$, vale a seguinte propriedade:

$$
\langle f, g\rangle_{N}=N\langle\hat{f}, \hat{g}\rangle_{N} .
$$


Demonstração.

$$
\begin{aligned}
\langle\hat{f}, \hat{g}\rangle_{N} & =\frac{1}{N} \sum_{w=-N / 2}^{N / 2-1} \frac{1}{N} \sum_{j=0}^{N-1} f_{j} e^{-2 \pi i w(j) / N} \frac{1}{N} \sum_{l=0}^{N-1} g_{l}^{*} e^{2 \pi i w(l) / N} \\
& =\left(\frac{1}{N}\right)^{3} \sum_{j=0}^{N-1} f_{j} \sum_{l=0}^{N-1} g_{l}^{*} \sum_{w=-N / 2}^{N / 2-1} e^{2 \pi i w(l-j) / N} \\
& =\frac{1}{N}\langle f, g\rangle_{N} .
\end{aligned}
$$

Teorema 1.2.14 (Identidade de Parseval). Para os conjuntos de valores $\left\{f_{l}\right\}_{l=0,1, \ldots, N-1}$, reais ou complexos, definidos sobre uma malha discreta no intervalo unitário $\left(\left\{x_{l}=l / N\right\}_{l=0,1, \ldots, N-1}\right)$, vale a seguinte propriedade:

$$
\|f\|_{N}=\sqrt{N}\|\hat{f}\|
$$

Demonstração. Consequência do Teorema de Plancherel (1.2.13).

Proposição 1.2.15 (Fórmula de Poisson). Seja $f$ uma função regular. Então

$$
\sum_{n=-\infty}^{\infty} f(2 n \pi)=\sum_{n=-\infty}^{\infty} \hat{f}(n)
$$

ou em outra forma

$$
\sum_{n=-\infty}^{\infty} f(x+n)=\sum_{n=-\infty}^{\infty} 2 \pi \hat{f}(n) e^{2 \pi i n x}
$$

Demonstração. Pode ser encontrada em inúmeros textos, cito aqui Chui (1992) e Wassermann (1999).

\subsection{Outros conceitos relevantes}

\subsubsection{Base de Riesz}

Definição 1.3.1. Dizemos que um conjunto de funções $B=\left\{b_{j}\right\}_{j \in \mathbb{Z}}$ é uma Base de Riesz de um espaço $V$ se o espaço gerado por estas funções é denso em $V$ e existem constantes positivas e finitas $A$ e $B$ tais que:

$$
A\left\|\left\{b_{j}\right\}\right\|_{l^{2}}^{2} \leq\left\|\sum_{j \in \mathbb{Z}} c_{j} b_{j}\right\|_{V}^{2} \leq B\left\|\left\{b_{j}\right\}\right\|_{l^{2}}^{2}
$$

para toda sequência quadrado somável

$$
\left\|\left\{c_{j}\right\}\right\|_{l^{2}}^{2}=\sum_{j \in \mathbb{Z}}\left|c_{j}\right|^{2}<\infty
$$


Definição 1.3.2. Seja $g \in U$, com $V \subset U$ e $B$ uma base de Riesz para $V$, como acima, então denotamos a projeção, $P$, de $g$ em $V$, por

$$
P_{V}(g)=\sum_{j \in \mathbb{Z}}\left\langle g, b_{j}\right\rangle b_{j}
$$

\subsubsection{Soma Direta $(\oplus)$}

Definição 1.3.3. Sejam $V$ e $U$ subespaços vetoriais de $L^{2}(\mathbb{R})$, então denotaremos pela soma direta entre $V$ e $U$,

$$
W=V \oplus U
$$

o sentido de que para todo $f \in W$ existe uma única decomposição da forma

$$
f(x)=g(x)+h(x)
$$

com $g \in V$ e $h \in U$.

\subsubsection{Função Indicadora}

Definição 1.3.4. Definimos o símbolo de Kronecker como função indicadora:

$$
\delta_{j k}= \begin{cases}1 & \text { se } j=k, \\ 0 & \text { se } j \neq k,\end{cases}
$$




\section{Capítulo 2}

\section{Ondaletas}

\subsection{Fundamentos}

Apresentamos a seguir alguns conceitos básicos referentes à construção de bases de ondaletas. Como referências básicas adotamos os livros de Daubechies (1992) e de Chui (1992).

De forma abreviada, uma ondaleta é uma função usada para decompor outras funções, ou sinais, de forma a tornar possível analisarmos estas outras funções, ou sinais, no domínio da frequência e espaço (ou tempo) conjuntamente.

A denominação "ondaleta" é a tradução para português da palavra inglesa "wavelet" mais comumente usada na literatura em português (MORETTIN, 1999), seguindo a origem francesa da palavra "ondelette" MEYER, 1986).

Adotaremos como a definição de ondaleta proposta em Chui (1992).

Definição 2.1.1. Uma função $\psi \in L^{2}(\mathbb{R})$ é denominada por ondaleta ortonormal, ou ondaleta mãe ortonormal, se

$$
\int \psi=0
$$

e o conjunto

$$
V=\left\{\psi_{j k}\right\}_{j, k \in \mathbb{Z}}
$$

onde

$$
\psi_{j k}=2^{j / 2} \psi\left(2^{j} x-k\right)
$$

for uma base ortonormal do $L^{2}(\mathbb{R})$.

Com isso podemos verificar as seguintes propriedades:

Proposição 2.1.2. Seja

$$
W_{j}=\operatorname{span}\left\{\psi_{j k}\right\}_{k \in \mathbb{Z}}
$$

o espaço vetorial gerado pela funções do nível $j$, e além disso definimos os espaços $V_{j}$ por

$$
V_{j}=\bigoplus_{k=-\infty}^{j-1} W_{k}:=\ldots \oplus W_{j-2} \oplus W_{j-1} .
$$


então teremos que:

1. $\ldots \subset V_{-1} \subset V_{0} \subset V_{1} \subset \ldots$,

2. $\overline{\left(\bigcup_{j \in \mathbb{Z}} V_{j}\right)}=L^{2}(\mathbb{R})$,

3. $\bigcap_{j \in \mathbb{Z}} V_{j}=\{0\}$,

4. $V_{j}=W_{j-1} \oplus V_{j-1}$,

5. $f(x) \in V_{j} \Leftrightarrow f(2 x) \in V_{j+1}, \quad j \in \mathbb{Z}$.

onde $\bar{A}$, para um subespaço $A$ qualquer, é o fecho de $A$ no espaço em questão $\left(L^{2}(\mathbb{R})\right)$.

Demonstração. A demonstração segue da definição de ondaleta, consulte Chui (1992), página 120, para detalhes.

Consideremos que o subespaço $V_{0}$ possa ser gerado por uma única função $\phi \in L^{2}(\mathbb{R})$ no sentido de que

$$
V_{0}=\overline{\operatorname{span}\left\{\phi_{0, k}: k \in \mathbb{Z}\right\}}
$$

onde

$$
\phi_{j k}=2^{j / 2} \phi\left(2^{j} x-k\right) .
$$

Definição 2.1.3. Uma função $\phi \in L^{2}(\mathbb{R})$ gera uma análise multi-resolução se ela gera uma sequência de espaços fechados $V_{j}$ que satisfazem as condições das propriedades 1, 2, 3 e 5 acima, e ainda que o conjunto $\left\{\phi_{0, k}\right\}$ forme uma base de Riesz de $V_{0}$. Se $\phi$ gera uma análise multi-resolução então ela é chamada de função escala, ou ondaleta pai.

Admitiremos como hipótese a respeito da função $\phi(x)$ o seguinte

$$
\int_{\mathbb{R}} \phi(x) d x=1
$$

Desta hipótese e de definição de análise multi-resolução, pode-se mostrar a propriedade a seguir, que é denominada por partição da unidade,

$$
\sum_{k \in \mathbb{Z}} \phi(x+k)=1
$$

Temos resultados análogos para $\psi$, que veremos a seguir.

Proposição 2.1.4. Se a ondaleta mãe $\psi$, com transladas e comprimidas/dilatadas $\psi_{j k}(x)=$ $2^{j / 2} \psi\left(2^{j} x-k\right)$, formam um conjunto ortogonal em $L^{2}(\mathbb{R})$, com $|\psi| \leq C(1+|x|)^{-m-1-\epsilon}, \psi \in \mathcal{C}^{m}(\mathbb{R})$, $\epsilon>0$ e $\psi^{(l)}$ limitada para todo $l \leq m$ então teremos que

$$
\int_{\mathbb{R}} x^{l} \psi(x) d x=0, \quad l=0,1, \ldots, m .
$$


Demonstração. A demonstração encontra-se no livro de Daubechies (1992), na página 154, e requer a definição de conceitos não abordados aqui e demonstrações de outros teoremas.

Agora se utilizarmos uma função ondaleta $\psi$, que denominaremos por ondaleta mãe quando for necessário fazer distinção com a ondaleta pai, e uma função escala, ou ondaleta pai, $\phi$, podemos construir uma base ortogonal para o $L^{2}(\mathbb{R})$. Assim, se $f \in L^{2}(\mathbb{R})$, então pode ser escrita como

$$
f(x)=\sum_{k \in \mathbb{Z}}\left\langle f, \phi_{0, k}\right\rangle \phi_{0, k}+\sum_{j=0}^{\infty} \sum_{k \in \mathbb{Z}}\left\langle f, \psi_{j, k}\right\rangle \psi_{j, k}
$$

Esta noção de Análise Multi-resolução, e sua teoria envolvida, foi primeiro introduzida por Meyer (1986), e Mallat (1988). Em 1992 Daubechies e Cohen desenvolvem a teoria para ondaletas em intervalos, que vamos analisar mais adiante.

\section{$2.2 \quad \operatorname{Em} L^{2}([0,1])$}

Nesta seção desenvolveremos um pouco da teoria envolvida na transformação de ondaletas em $L^{2}(\mathbb{R})$ para o $L^{2}([0,1])$.

Definição 2.2.1. Dada uma análise multi-resolução com função escala $\phi$ e ondaleta $\psi$, ambas contínuas com decaimento da ordem de

$$
|\phi(x)|,|\psi(x)| \leq C(1+|x|)^{-(2+\epsilon)}
$$

com $\epsilon>0$, definimos por ondaletas periodizadas as funções

$$
\phi_{j k}^{p e r}(x)=\sum_{l \in \mathbb{Z}} \phi_{j k}(x+l)
$$

e

$$
\psi_{j k}^{p e r}(x)=\sum_{l \in \mathbb{Z}} \psi_{j k}(x+l)
$$

e ainda definimos os espaços

$$
\begin{aligned}
V_{j}^{\text {per }} & =\overline{\operatorname{span}\left\{\phi_{j k}^{\text {per }} ; k \in \mathbb{Z}\right\}}, \\
W_{j}^{\text {per }} & =\overline{\operatorname{span}\left\{\psi_{j k}^{\text {per }} ; k \in \mathbb{Z}\right\}} .
\end{aligned}
$$

A primeira observação é que claramente as funções definidas acima são periódicas com período um. Vejamos,

$$
\phi_{j k}^{p e r}(x+1)=\sum_{l \in \mathbb{Z}} \phi_{j k}(x+1+l)=\sum_{m \in \mathbb{Z}} \phi_{j k}(x+m)=\phi_{j k}^{p e r}(x),
$$

e o mesmo pode ser feito para $\psi^{\text {per }}$. Chamaremos de $\bar{V}_{j}$ o fecho de $V_{j}$ em $L^{2}([0,1])$, e de $P_{j}$ o espaço das funções periódicas, com período 1 , pertencentes ao $\bar{V}_{j}$.

Lema 2.2.2. Se $j \leq 0$, então $P_{j}=\operatorname{span}\{1\}$, isto é, $P_{j}$ é o conjunto de funções constantes. 
Demonstração. Como $P_{j} \subset P_{j+1}$, pois $V_{j} \subset V_{j+1}$, então basta mostrar para $P_{0}$. Usaremos aqui nossa hipótese (2.3), e sua consequência (2.4), a respeito das características de $\phi$. O espaço $V_{0}$ é gerado por $\left\{\phi_{0, k}=\phi(x-k)\right\}_{k \in \mathbb{Z}}$, portanto, tome $f \in P_{0}$, escrevendo na base de $V_{0}$,

$$
f=\sum_{k \in \mathbb{Z}} c_{k} \phi(x-k)
$$

onde,

$$
c_{k}=\int_{-\infty}^{+\infty} f(x) \phi(x-k) d x=\int_{-\infty}^{+\infty} f(y+k) \phi(y) d y=\int_{-\infty}^{+\infty} f(y) \phi(y) d y=c_{0} .
$$

Ou seja, $f$ é constante,

$$
f=c_{0} \sum_{k \in \mathbb{Z}} \phi(x-k)=c_{0}
$$

Lema 2.2.3. Se $j>0, P_{j}$ tem dimensão $2^{j}$.

Demonstração. Seja $f \in P_{j}$,

$$
f=\sum_{k \in \mathbb{Z}} c_{j, k} \phi_{j, k}(x)
$$

Note que

$$
\phi_{j, k+m 2^{j}}^{p e r}(x)=\sum_{l \in \mathbb{Z}} \phi_{j, k+m 2^{j}}(x+l)=\sum_{l \in \mathbb{Z}} 2^{j / 2} \phi\left(2^{j}(x+l)-k-m 2^{|j|}\right)=\phi_{j, k}^{p e r}(x) .
$$

Como $f$ é periódica, então

$f(x+l)=\sum_{k \in \mathbb{Z}} c_{j, k} \phi_{j, k}((x+l))=2^{j / 2} \sum_{k \in \mathbb{Z}} c_{j, k} \phi\left(2^{j} x+\left(2^{j} l-k\right)\right)=2^{j / 2} \sum_{p \in \mathbb{Z}} c_{j, p-2^{j} l} \phi\left(2^{j} x-p\right)=\sum_{p \in \mathbb{Z}} c_{j, p-2^{j} l} \phi_{j, p}(x)$,

resultando que $c_{j, k \pm 2^{j} l}=c_{j, k}$. Com isso ficamos com

$$
f(x)=\sum_{k=0}^{2^{j}-1} c_{j, k}\left(\sum_{l \in \mathbb{Z}} \phi_{j, k+2^{j} l}(x)\right)=\sum_{k=0}^{2^{j}-1} c_{j, k} \phi_{j, k}^{\text {per }}(x) .
$$

Portanto $f$ pode ser escrita como combinação linear de $2^{j}$ funções periódicas de período 1 , ortogonais, encerrando a demonstração.

Proposição 2.2.4. O conjunto de funções

$$
\left\{1, \psi_{j k}^{p e r}\right\}_{j=0,1, \ldots, \quad k=0,1, \ldots, 2^{j}-1}
$$

forma uma base ortonormal para o $L^{2}([0,1])$.

Demonstração. Primeiro note que o elemento da base $\{1\}$ provem de $\phi_{0, k}^{p e r}$, com isso, como visto na demonstração do lema anterior, temos que $V_{0}^{\text {per }}=P_{0}$. Sejam $Q_{j}$ os complementos ortogonais de $P_{j}$ 
em $P_{j+1}$, então, por construção da análise multi-resolução, temos como base para $Q_{j}$ os elementos $\psi_{j k}^{\text {per }}$, e que portanto $Q_{j}=W_{j}^{\text {per }}$. Resultando portanto que,

$$
L^{2}([0,1])=\overline{\left\{V_{0}^{p e r}, W_{0}^{p e r}, W_{1}^{p e r}, \ldots\right\}}
$$

que encerra a demonstração.

Estas demonstrações e mais detalhes sobre ondaletas periodizadas podem ser encontrados na página 552 do artigo de Jaffard e Laurençot (1992), ou na página 304 do livro de Daubechies (1992).

\subsection{Exemplos}

Mostraremos a seguir alguns exemplos de ondaletas pertinentes, junto com figuras da sua função ondaleta pai $(\phi)$ e mãe $(\psi)$.

\subsubsection{Haar}

Esta foi a primeira base de ondaletas conhecida, desenvolvida por Haar (1911).

$$
\begin{gathered}
\psi(t)= \begin{cases}1 & 0 \leq t<1 / 2, \\
-1 & 1 / 2 \leq t<1 \\
0 & \text { caso contrário. }\end{cases} \\
\phi(t)= \begin{cases}1 & 0 \leq t<1, \\
0 & \text { caso contrário. }\end{cases}
\end{gathered}
$$
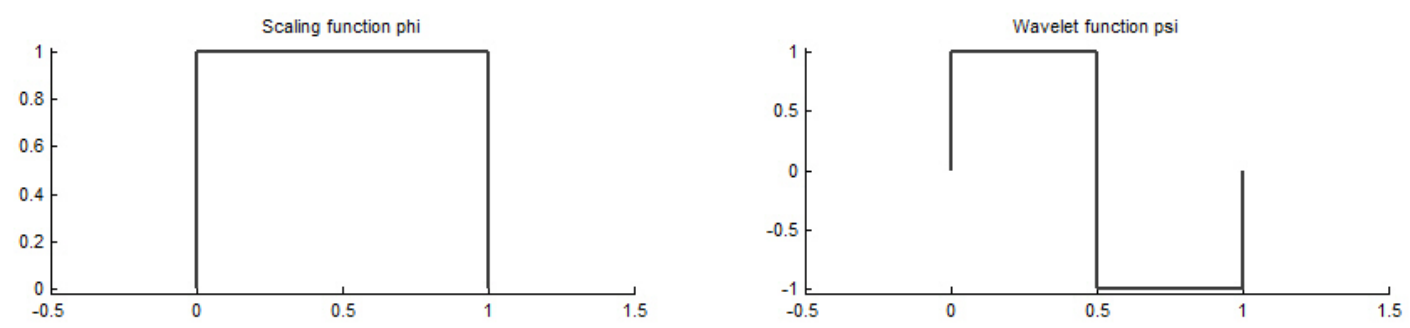

Figura 2.1: Ondaletas de Haar: Pai $(\phi)$ e Mãe $(\psi)$

Esta base é interessante para compreendermos um pouco mais sobre ondaletas. Se considerarmos uma função $f$ real contínua restrita ao intervalo fechado $[0,1]$, e a base $B=\left\{\phi, \psi_{j k}\right\}_{j=0,1, \ldots k=0,1 \ldots, 2^{j}-1}$ então teremos que

$$
P_{B}(f)(x)=a_{0} \phi(x)+\sum_{j=0}^{\infty} \sum_{k=0}^{2^{j}-1} a_{2^{j}+k} \psi_{j k}
$$


onde

$$
\begin{gathered}
a_{0}=\langle f, \phi\rangle \\
a_{2^{j}+k}=\left\langle f, \psi_{j k}\right\rangle
\end{gathered}
$$

O índice $j$ denota escalas, e o índice $k$ denota translações. A função $\phi$ é constante, então atua na projeção indicando o nível médio de $f$. O primeiro nível de ondaletas $(j=0)$ define a variação entre os subintervalos $[0,1 / 2]$ e $[1 / 2,1]$. Os próximos níveis definem variações da função em intervalos menores, indicando maiores detalhes da função. Note que no nível $j=0$ há apenas uma função ondaleta mãe, no nível $j=1$, temos 2 elementos da base, no próximo nível temos 4 elementos da base, e assim sucessivamente. Quanto mais níveis tivermos mais próxima a projeção ficará da função a ser aproximada.

A ondaleta de Haar é pouco usada na resolução de EDPs, já que não é uma função regular, mas foi utilizada no trabalho de Lepik (2007).

\subsubsection{Daubechies}

As ondaletas de Daubechies definem toda uma classe de ondaletas ortogonais com suporte compacto. Têm ainda a propriedade de ter momentos nulos até um certo parâmetro $n$, isto é,

$$
\int x^{n} \psi(x) d x=0
$$

onde $\psi(x)$ é uma ondaleta de Daubechies, e recebem a notação $\mathrm{D}(2 \mathrm{n})$, ou $\mathrm{Db}(2 \mathrm{n})$. A ondaleta de Haar é na verdade um caso particular, a D2. Mostramos na figura 2.2 a ondaleta D4, que tem 2 momentos nulos, ideais, por exemplo, para a representação de polinômios quadráticos.
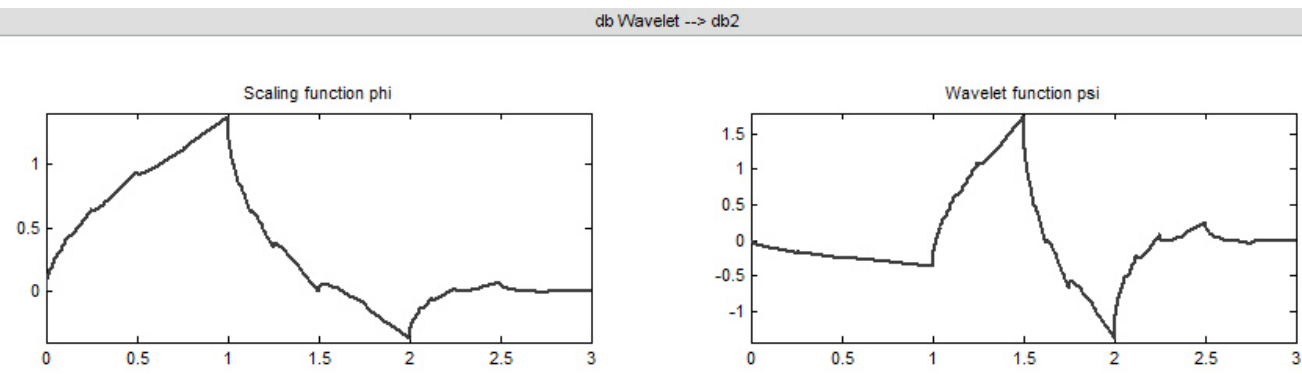

Figura 2.2: Ondaletas de Daubechies (dB4): Pai $(\phi)$ e Mãe $(\psi)$

As ondaletas de Daubechies não podem ser escritas com expressão analítica, isto é, não têm forma fechada de escrita. Porém possuem um algoritmo de cascata para sua construção. Este fato dificulta o manuseio de expressões de funções escritas nesta base, por exemplo em problemas de equações diferenciais. Seu uso está bastante atrelado a problemas de análise de sinais, mas diversos pesquisadores têm usado esta base na resolução de EDPs, tanto com abordagem de adaptatividade de malha (BACRY; MALLAT; PAPANICOLAOU, 1992; DOMINGUES, 2001), quanto em métodos de Galerkin (BESORA, 2004; HO; YANG, 2001; SCHULT; WYLD, 1992; AMARATUNGA et al., 1994). O 
trabalho de Besora (2004) analisa um método de Galerkin-Ondaleta numérico para resolução da equação da onda, mas ressalta os elevados números de condição da matriz utilizada no método. Em Schult e Wyld (1992) é feita uma análise sobre o método de Galerkin com ondaletas de Daubechies na resolução da equação de Burgers, concluindo que para o caso unidimensional há um elevado custo computacional quando comparado com métodos de diferenças finitas.

\subsubsection{Meyer}

Destacamos aqui as ondaletas de Meyer por terem características mais próximas das ondaletas que serão utilizadas nesta dissertação, as ondaletas harmônicas. As ondaletas de Meyer, apresentadas na figura 2.3, não têm suporte compacto, mas possuem forma fechada de escrita e regularidade. Foram utilizadas no trabalho de Lopes e Mattos (2003) para resolução de EDPs com coeficientes variáveis, da forma,

$$
\begin{aligned}
K(x) u_{x x}(x, t) & =u_{t}(x, t), \quad t \geq 0, x \in[0,1] \\
u(0, x) & =g(x), \quad u_{x}(0, x)=0,
\end{aligned}
$$

onde $u \in L^{2}\left([0,1] \times \mathbb{R}^{+}\right)$e $0<K<\infty$ função contínua. Utilizaram um método de GalerkinOndaleta e obtiveram resultados analíticos de convergência e estabilidade, mas não realizaram experimentos numéricos.

meyr Wavelet $\rightarrow$ meyr
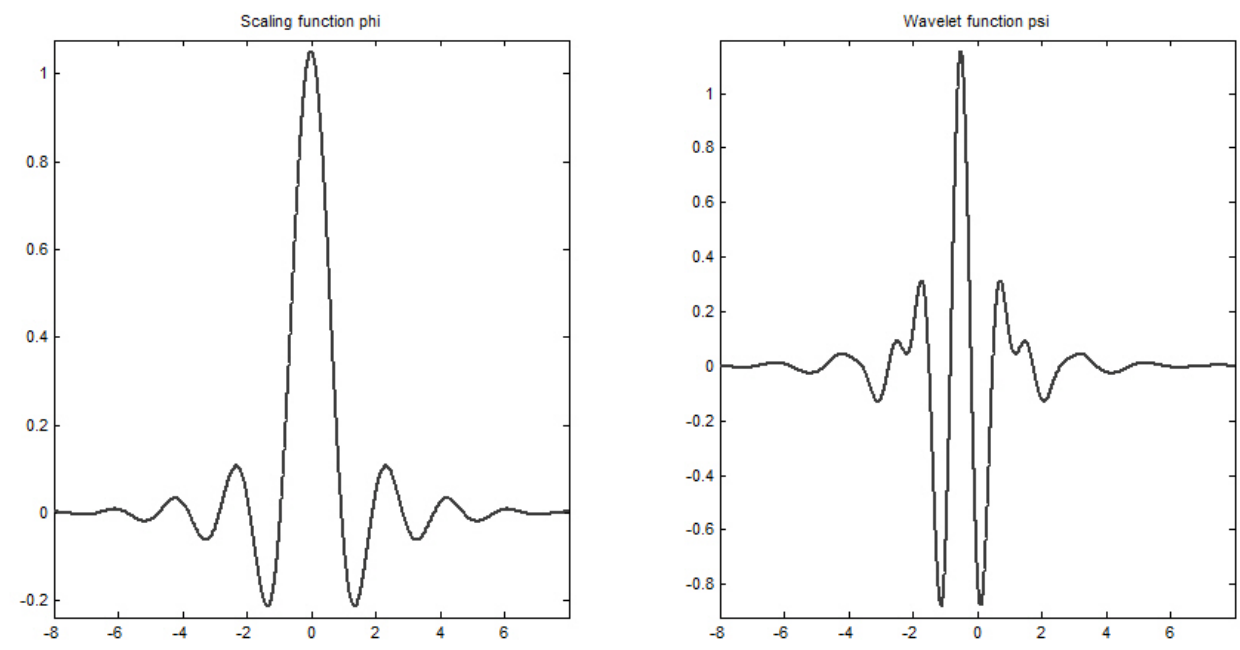

Figura 2.3: Ondaletas de Meyer: Pai $(\phi)$ e Mãe $(\psi)$

\subsection{Ondaletas harmônicas}

As ondaletas harmônicas foram primeiro definidas por Newland (1993). Newland detalha neste trabalho de 1993 as propriedades e algoritmos para implementação do método de transformada 


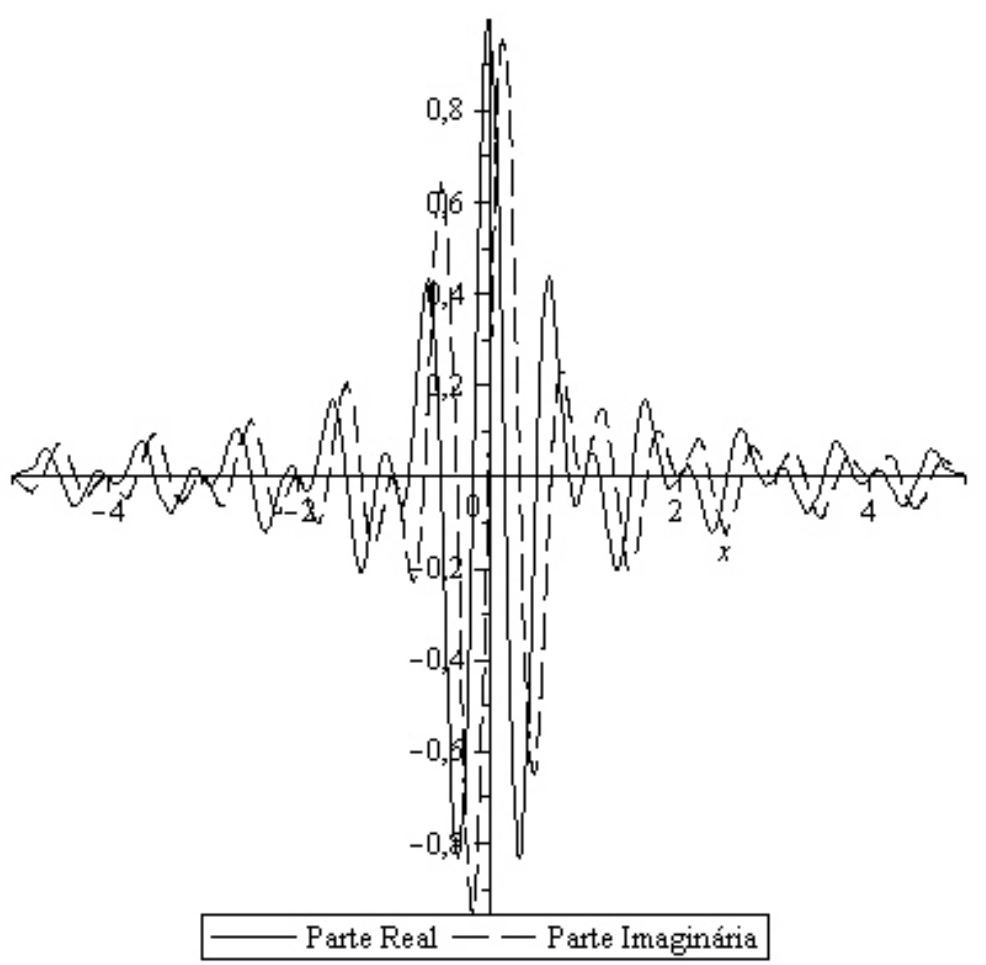

Figura 2.4: Ondaleta harmônica em $\mathbb{R}(\psi(x))$

rápida de ondaletas harmônicas. Dentre suas características, destaca-se por ser uma função sem suporte compacto, mas com suporte compacto no domínio de frequências. Além disso esta ondaleta possui forma explícita, e é diferenciável, o que facilita seu uso em problemas ligados a EDPs (CATTANI, 2003; CATTANI, 2005; CATTANI, 2008; MUNIANDY; MOROZ, 1997). Vamos mostrar algumas características mais relevantes e interessantes.

\subsection{1 $\operatorname{Em~} L^{2}(\mathbb{R})$}

Definição 2.4.1. Uma ondaleta harmônica para o espaço $L^{2}(\mathbb{R})$, é uma função complexa dada por

$$
\psi(x)=\frac{\left(e^{4 i \pi x}-e^{2 i \pi x}\right)}{2 \pi i x}
$$

As partes real e imaginária são apresentadas na figura 2.4.

Proposição 2.4.2. A transformada de Fourier de uma ondaleta harmônica, com base na definição (1.2.1), resulta em

$$
\hat{\psi}(\omega)= \begin{cases}\frac{1}{2 \pi} & \text { se } 2 \pi \leq \omega<4 \pi \\ 0 & \text { caso contrário. }\end{cases}
$$

Demonstração. Se tomarmos a transformada inversa de Fourier de $\hat{\psi}$, com base na definição 1.2 .2 , 


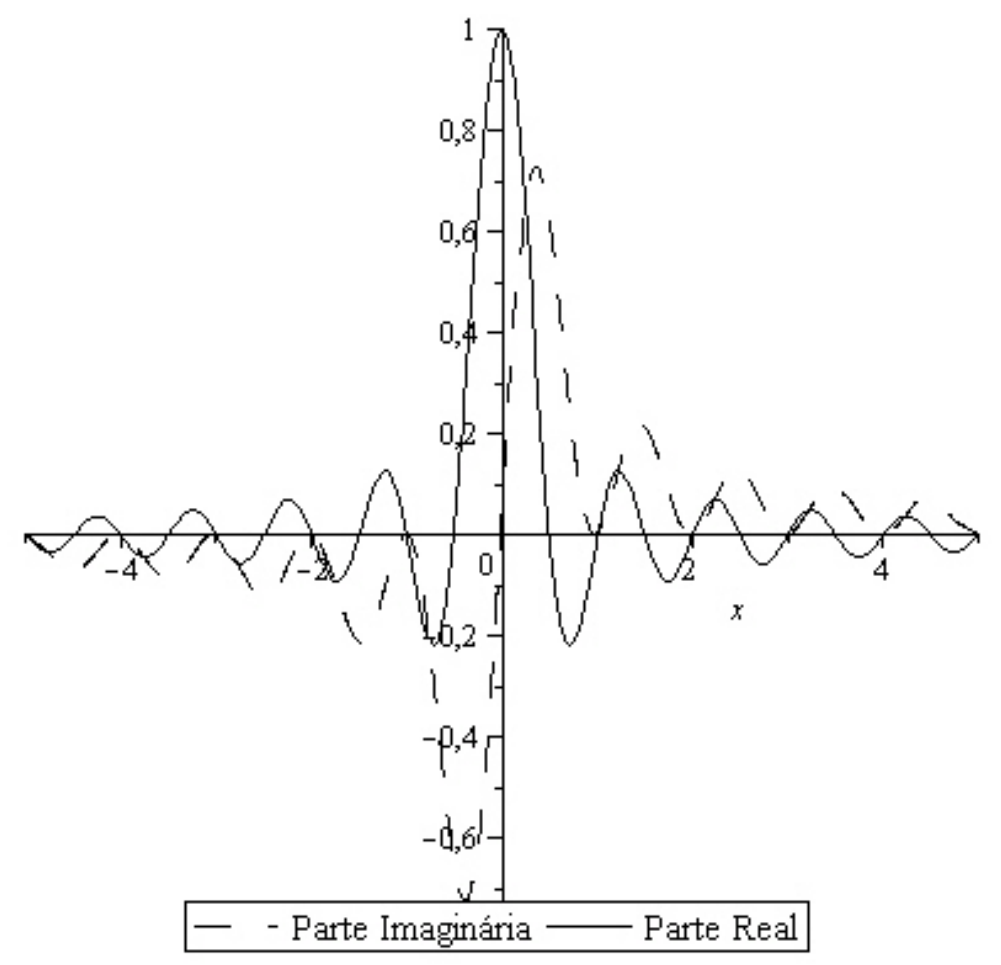

Figura 2.5: Ondaleta harmônica pai em $\mathbb{R}(\phi(x))$

chegamos na expressão de $\psi$ dada na definição 2.4.1 (NEWLAND, 1993). Daí segue da equação (1.5).

Note que portanto a ondaleta harmônica tem suporte compacto no domínio de frequências e que tem decaimento da ordem de $1 / x$ no domínio do espaço.

Definição 2.4.3. A função escala referente à ondaleta harmônica, também denominada ondaleta harmônica pai, para o espaço $L^{2}(\mathbb{R})$, é dada pela função complexa

$$
\phi(x)=\frac{\left(e^{2 i \pi x}-1\right)}{2 \pi i x} .
$$

As partes real e imaginária são apresentadas na figura 2.5.

Proposição 2.4.4. A transformada de Fourier de uma ondaleta harmônica pai, ou função escala, com base na definição (1.2.1), é dada por:

$$
\hat{\phi}(\omega)= \begin{cases}\frac{1}{2 \pi} & \text { se } 0 \leq \omega<2 \pi \\ 0 & \text { caso contrário. }\end{cases}
$$


Demonstração. Análogo ao caso anterior 2.4.1). Se tomarmos a transformada inversa de Fourier de $\hat{\phi}$, com base na definição 1.2 .2 , chegamos na expressão de $\phi$ dada na definição 2.4.3) NEWLAND, 1993).

Pode-se também estabelecer uma relação entre as ondaletas harmônicas pai e mãe, que fica dada por

$$
\psi(x)=e^{2 \pi i x} \phi(x)
$$

Definição 2.4.5. Consideramos as ondaletas harmônicas transladas e dilatadas/comprimidas, já normalizadas, e usando a forma usual, o conjunto de funções

$$
\psi_{j k}=2^{j / 2} \psi\left(2^{j} x-k\right)=2^{j / 2} \frac{e^{4 \pi i\left(2^{j} x-k\right)}-e^{2 \pi i\left(2^{j} x-k\right)}}{2 \pi i\left(2^{j} x-k\right)}
$$

$\operatorname{com} j, k \in \mathbb{Z}$.

Definição 2.4.6. Vamos considerar como funções escala, ou ondaletas harmônicas pais, transladas, já normalizadas, o conjunto de funções

$$
\phi_{0, k}(x)=\phi_{k}(x)=\phi(x-k)
$$

para $k \in \mathbb{Z}$.

Como vamos considerar espaços que gerem uma Análise Multi-resolução, ou seja, adotaremos a função escala como geradora do espaço $V_{0}$, e utilizaremos as ondaletas apenas para $V_{j}$ com $j \geq 0$, então podemos olhar para $\psi_{j k}$ para $j \geq 0$ apenas.

Proposição 2.4.7. Uma função de ondaleta harmônica $\psi_{j k}$ terá transformada de Fourier dada por

$$
\hat{\psi_{j k}}(\omega)= \begin{cases}\frac{1}{2 \pi 2^{j / 2}} e^{\frac{-2 \pi i \omega k}{2^{j}}} & \text { se } 2 \pi 2^{j} \leq \omega<2 \pi 2^{j+1} \\ 0 & \text { caso contrário. }\end{cases}
$$

Demonstração. Decorrência de (2.4.1) e da propriedade (1.1) da transformada de Fourier.

Proposição 2.4.8. Uma função escala de ondaleta harmônica $\phi_{k}$ terá transformada de Fourier com suporte definido no intervalo $[0,2 \pi]$.

Demonstração. Decorrência da transformada de Fourier de $\phi$ 2.4.1 e da propriedade de translação da transformada de Fourier 1.1.

Novamente nota-se a característica de compacidade do suporte, e mais, note que, para $j$ s diferentes, o suporte não tem intersecção.

Proposição 2.4.9. O conjunto de funções $\psi_{j k}$ é ortogonal em relação ao produto interno usual do $L^{2}(\mathbb{R})$, definido em (1.1.1), isto é,

$$
\left\langle\psi_{j k}, \psi_{p q}\right\rangle_{L^{2}(\mathbb{R})}=\delta_{j p} \delta_{k q}
$$


com $j, p \in \mathbb{N}$ e $k, q \in \mathbb{Z}$.

Demonstração. Pela identidade de Parseval (1.2.3) basta mostrarmos que

$$
2 \pi\left\langle\widehat{\psi_{j k}}, \widehat{\psi_{p q}}\right\rangle_{L^{2}(\mathbb{R})}=\delta_{j p} \delta_{k q}
$$

Note que se $p \neq j$ então os suportes de $\widehat{\psi_{j k}}$ e $\widehat{\psi_{p q}}$ tem intersecção vazia, e portanto o produto interno será nulo. Vamos analisar o caso $p=j$.

$$
\begin{aligned}
\left\langle\psi_{j k}, \psi_{j q}\right\rangle_{L^{2}(\mathbb{R})} & =2 \pi\left\langle\widehat{\psi_{j k}}, \widehat{\psi_{j q}}\right\rangle_{L^{2}(\mathbb{R})} \\
& =2 \pi \int_{-\infty}^{+\infty} \widehat{\psi_{j k}}(\omega){\widehat{\psi_{j q}}}^{*}(\omega) d \omega \\
& =\frac{1}{2 \pi 2^{j}} \int_{2 \pi 2^{j}}^{4 \pi 2^{j}} e^{\frac{i \omega(q-k)}{2^{j}}} d \omega \\
& =\delta_{k q}
\end{aligned}
$$

Portanto $\left\langle\psi_{j k}, \psi_{p q}\right\rangle_{L^{2}(\mathbb{R})}=\delta_{j p} \delta_{k q}$.

Proposição 2.4.10. O conjunto de funções $\left\{\phi_{k}, \psi_{j k}\right\}_{k \in \mathbb{Z}, j \geq 0}$ é ortogonal em relação ao produto interno usual do $L^{2}(\mathbb{R})$, definido em 1.1.1. Além disso o conjunto é denso em $L^{2}(\mathbb{R})$

Demonstração. A ortogonalidade pode ser verificada diretamente fazendo uso do teorema de Plancherel 1.2.3. Verifica-se que os coeficientes de ondaletas harmônicas satisfazem a identidade de Parseval, o restante da demonstração segue disso. Para a demonstração completa verifique o apêndice C do trabalho de Newland (1993).

\subsection{2 $\operatorname{Em} L^{2}([0,1])$}

Definição 2.4.11. Definimos por ondaleta harmônica para o intervalo [0,1], ou equivalentemente ondaleta harmônica periodizada, de nível $j$ e translação $k$ o seguinte:

$$
\psi_{j k}(x)=2^{-j / 2} \sum_{m_{j}=2^{j}}^{2^{j+1}-1} e^{2 \pi i m_{j}\left(x-\frac{k}{2^{j}}\right)}
$$

Esta ondaleta pode ser deduzida a partir da ondaleta harmônica para o $L^{2}(\mathbb{R})$ utilizando-se da técnica de periodização proposta por Daubechies (1992), mostrada na seção 2.2). Para evitar notação excessiva tiramos a denotação per das funções $\psi$, mas deve-se manter subentendido que tratam-se de ondaletas periodizadas. Mostraremos a seguir sua dedução, que está fundamentada na fórmula de Poisson 1.2.15 e na transformada de Fourier de $\psi_{j k}$. 


$$
\begin{aligned}
\psi_{j k}^{p e r} & =\sum_{l \in \mathbb{Z}} \psi_{j k}(x+l)=2 \pi \sum_{l \in \mathbb{Z}} \hat{\psi_{j k}}(l) e^{2 \pi i l x} \\
& =\sum_{l=2^{j}}^{2^{j+1}-1} 2^{-j / 2} e^{\frac{-2 \pi i l k}{2^{j}}} e^{2 \pi i l x} \\
& =2^{-j / 2} \sum_{l=2^{j}}^{2^{j+1}-1} e^{2 \pi i l\left(x-\frac{k}{2^{j}}\right)} .
\end{aligned}
$$

Conforme descrito na seção (2.2), a função escala $\phi$ será constante, e para termos uma base normalizada adotamos

$$
\phi(x)=1
$$

Mostramos na figura 2.6 gráficos de ondaletas harmônicas periodizadas, com sua parte real e imaginária, para algumas escalas, $j \mathrm{~s}$, e translações, $k \mathrm{~s}$, usamos $N=2^{8}$ pontos para fazer o gráfico. Primeiro podemos observar que no nível zero $(j=0)$, para o qual só temos o elemento de translação $(k=0)$, temos exatamente uma função cosseno, para a parte real, e seno, para parte imaginária. Do nível 1 ao nível 4 observamos que temos funções suaves que tendem a ficar localizadas no espaço com o aumento dos níveis.

Outra observação importante neste instante é percebermos que na verdade as ondaletas harmônicas periodizadas nada mais são que uma mudança de base em relação à base clássica de Fourier, com o propósito de obtermos uma propriedade de localidade no espaço. Calculando as transformadas de fourier de $\psi_{j k}$, teremos:

$$
\begin{aligned}
\widehat{\psi_{j k}}(w) & =\int_{0}^{1} 2^{-j / 2} \sum_{m_{j}=2^{j}}^{2^{j+1}-1} e^{2 \pi i m_{j}\left(x-\frac{k}{2^{j}}\right)} e^{-2 \pi i w x} d x \\
& =2^{-j / 2} \sum_{m_{j}=2^{j}}^{2^{j+1}-1} e^{-2 \pi i m_{j} \frac{k}{2^{j}}} \int_{0}^{1} e^{2 \pi i\left(m_{j}-w\right) x} d x \\
& = \begin{cases}2^{-j / 2} e^{-2 \pi i w \frac{k}{2^{j}}} & \text { quando } m_{j}=w=2^{j}, \ldots, 2^{j+1}-1, \\
0 & \text { caso contrário, }\end{cases}
\end{aligned}
$$

onde usamos as propriedades da transformada de Fourier vistas na seção (1.2). Apresentamos na figura 2.7 as transformadas de Fourier para as ondaletas harmônicas.

Note que para cada nível $j$ o suporte da transformada fica limitado à um intervalo $\left[2^{j}, 2^{j+1}-1\right]$, resultando na cobertura dos números de onda de $1, \ldots, N / 2-1$, onde $N$ representa o número de pontos da malha que será sempre da forma

$$
N=2^{n}
$$



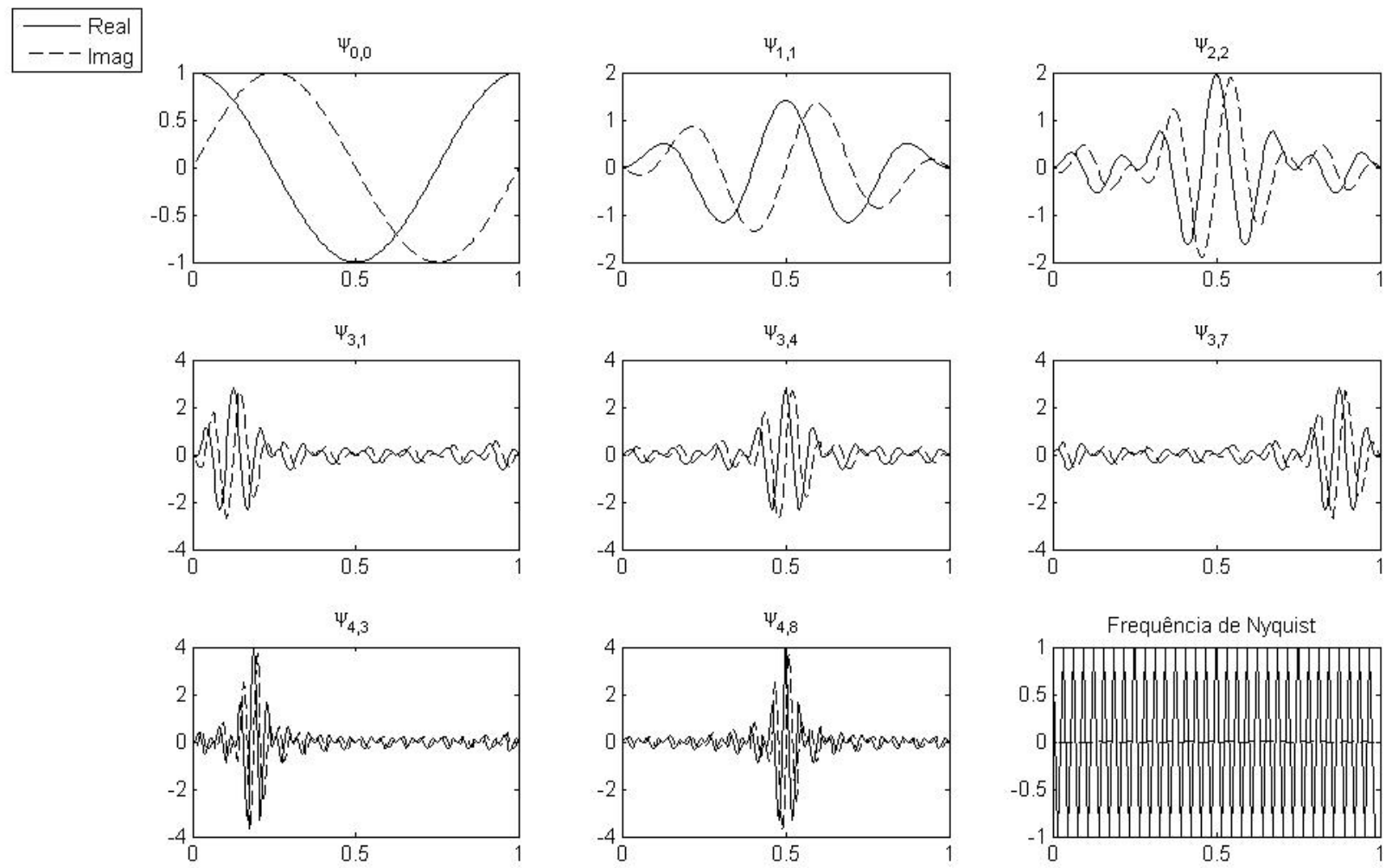

Figura 2.6: Ondaletas harmônicas $\psi_{j k}$ para o intervalo $[0,1]$

Para preservarmos a equivalência do espaço gerado pelas ondaletas harmônicas em relação ao espaço gerado pelos senos e cossenos de Fourier precisamos olhar para os números de onda a serem incluídos na base:

- $\psi_{j k}^{*}$ para incluir os números de onda de $-N / 2+1,-N / 2+2, \ldots,-1$.

- $\phi$ para incluir o número de onda 0.

- $\psi_{j k}$ para incluir os números de onda de $1,2, \ldots, N / 2-1$.

- A frequência de Nyquist, com número de onda $N / 2$, ou equivalente $-N / 2$, para isso incorporamos na base a função

$$
\psi_{n-1}=e^{-\frac{N}{2} 2 \pi i x}
$$

Com isso, montando uma base

$$
B_{n}=\left\{\phi, \psi_{j k}, \psi_{j k}^{*}, \psi_{n-1}\right\}_{j=0,1, \ldots, n-2 ; \quad k=0,1, \ldots, 2^{j}-1}
$$

cobrimos todos os números de onda e garantimos equivalência com o espaço gerado pela base de 

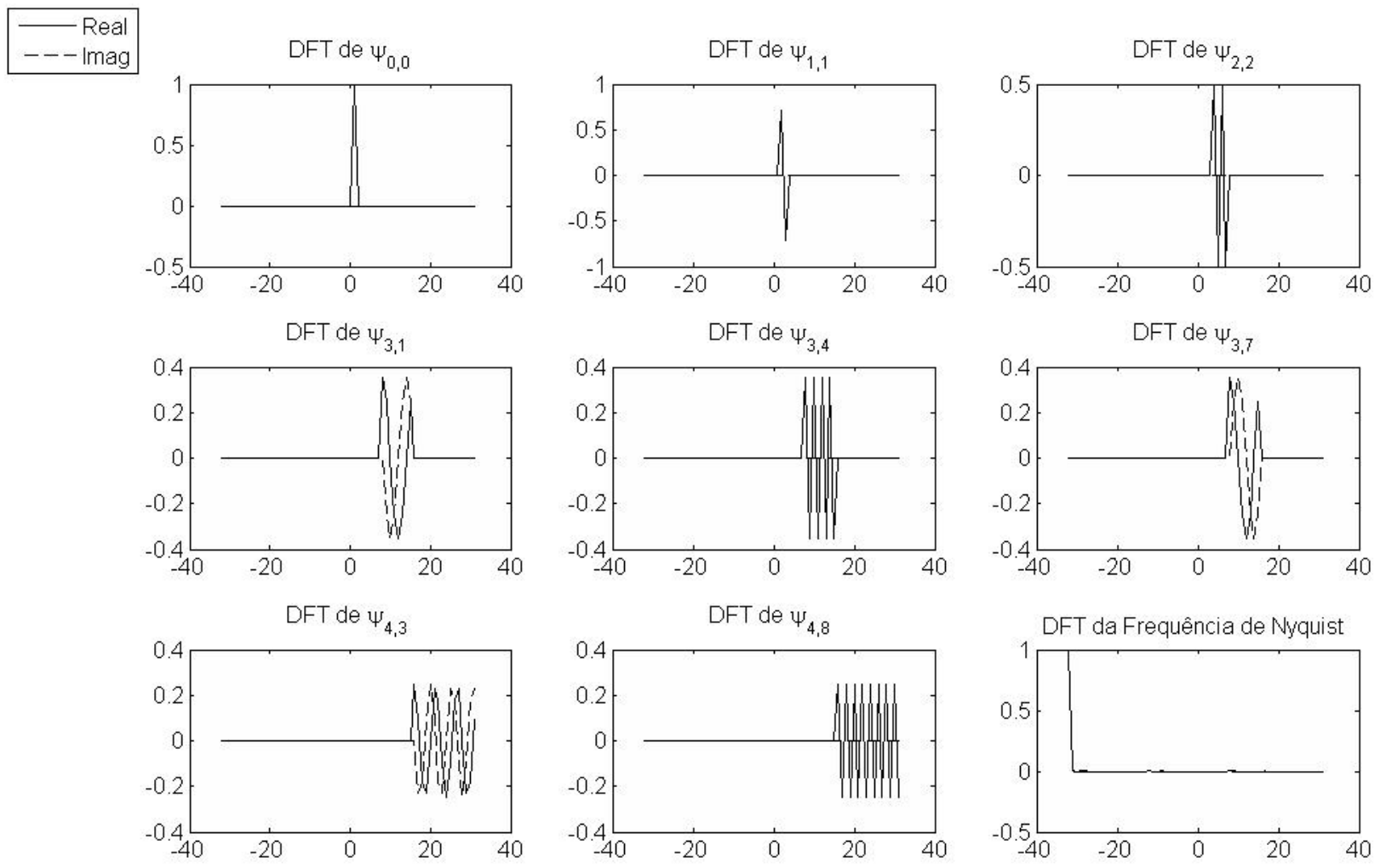

Figura 2.7: Transformada discreta de Fourier (DFT) das ondaletas harmônicas $\psi_{j k}$ para o intervalo [0,1].

Fourier $\left\{e^{2 \pi i j x}\right\}_{j=-N / 2, \ldots, N / 2-1}$, já que as funções $\psi_{j, k}(x)$ são combinações lineares de elementos da base de Fourier. De fato constituem apenas uma mudança de base para o mesmo espaço.

Vamos verificar agora se a base é ortonormal. Nos casos de elementos da base com suporte das transformadas de Fourier sem intersecção temos prontamente a ortogonalidade, isso ocorre nos casos: $\left\langle\phi(x), \psi_{j, k}(x)\right\rangle,\left\langle\phi(x), \psi_{j, k}^{*}(x)\right\rangle,\left\langle\phi(x), \psi_{n-1}(x)\right\rangle,\left\langle\psi_{j, k}(x), \psi_{p, q}^{*}(x)\right\rangle,\left\langle\psi_{j, k}(x), \psi_{n-1}(x)\right\rangle \mathrm{e}$ $\left\langle\psi_{j, k}^{*}(x), \psi_{n-1}(x)\right\rangle$. Usando as propriedades da transformada discreta de Fourier temos as outras relações:

$$
\langle\phi(x), \phi(x)\rangle=\langle 1,1\rangle=\int_{0}^{1} 1 d x=1
$$




$$
\begin{aligned}
& \left\langle\psi_{j, k}(x), \psi_{r, s}(x)\right\rangle=\int_{0}^{1} 2^{-j / 2} \sum_{m_{j}=2^{j}}^{2^{j+1}-1} e^{2 \pi i m_{j}\left(x-\frac{k}{2^{j}}\right)} 2^{-r / 2} \sum_{m_{r}=2^{r}}^{2^{r+1}-1} e^{-2 \pi i m_{r}\left(x-\frac{s}{2^{r}}\right)} d x \\
& =2^{-(j+r) / 2} \sum_{m_{j}=2^{j}}^{2^{j+1}-1} \sum_{m_{r}=2^{r}}^{2^{r+1}-1} e^{2 \pi i\left(\frac{m_{r} s}{2^{r}}-\frac{m_{j} k}{2^{j}}\right)} \int_{0}^{1} e^{2 \pi i\left(m_{j}-m_{r}\right) x} d x \\
& =2^{-(j+r) / 2} \sum_{m_{j}=2^{j}}^{2^{j+1}-1} \sum_{m_{r}=2^{r}}^{2^{r+1}-1} e^{2 \pi i\left(\frac{m_{r} s}{2^{r}}-\frac{m_{j} k}{2^{j}}\right)} \delta_{j, r} \\
& =2^{-j} \sum_{m_{j}=2^{j}}^{2^{j+1}-1} e^{2 \pi i\left(\frac{m_{j} s}{2^{j}}-\frac{m_{j} k}{2^{j}}\right)} \delta_{j, r} \\
& =\delta_{k, s} \delta_{j, r}, \quad j=0,1, \ldots, n-2, \quad k=0,1, \ldots, 2^{j}-1, \\
& \left\langle\psi_{j, k}^{*}(x), \psi_{r, s}^{*}(x)\right\rangle=\left\langle\psi_{r, s}(x), \psi_{j, k}(x)\right\rangle \\
& =\delta_{k, s} \delta_{j, r}, \quad j=0,1, \ldots, n-2, \quad k=0,1, \ldots, 2^{j}-1, \\
& \left\langle\psi_{n-1}(x), \psi_{n-1}(x)\right\rangle=\int_{0}^{1} e^{N \pi i x} e^{-N \pi i x} d x=1,
\end{aligned}
$$

O espaço gerado pela base ortonormal $B_{n}$ terá dimensão $N=2^{n}$, igual ao número de pontos da malha discretizada $\left\{x_{l}\right\}_{(l=0,1, \ldots, N-1)}$. Além disso, se $V_{n}=\operatorname{span}\left\{B_{n}\right\}$ então, por construção, teremos que

$$
\varlimsup_{n \rightarrow \infty} V_{n}=L^{2}([0,1]) .
$$

Naturalmente se estamos pensando em usar ondaletas em alguma aplicação vamos utilizar suas propriedades de localidade no espaço. Porém as ondaletas harmônicas não têm suporte compacto, mas tem um decaimento da ordem de $1 /|x|$. De acordo com o trabalho de Muniandy e Moroz (1997), o suporte de $\psi_{j, k}(x)$ está concentrado no intervalo $I_{j k}=\left[\frac{k-1}{2^{j}}, \frac{k+1}{2^{j}}\right]$. Neste estudo vamos generalizar esta hipótese considerando o seguinte suporte para $\psi_{j, k}(x)$ :

$$
I_{j k}^{d}=\left[\frac{k-d}{2^{j}}, \frac{k+d}{2^{j}}\right]
$$

para $d=1,2,3, \ldots$ Nos casos em que $k$ está perto das bordas, por exemplo $k=0$ ou $k=2^{j}-1$, deve-se considerar fronteiras periódicas $\left(\bmod \left(I_{j k}^{d}, 1\right)\right)$. Realizamos uma bateria de testes numéricos para verificar o percentual de área de $\psi_{j, k}(x)$ concentrada em $I_{j k}^{d}$. Para tanto tomamos $N=2^{17} \mathrm{e}$ 
estimamos a razão

$$
R_{j}^{d}=\frac{\left\|\psi_{j, k}(x)\right\|_{I_{j k}^{d}}}{\left\|\psi_{j, k}(x)\right\|_{[0,1]}}=\left\|\psi_{j, k}(x)\right\|_{I_{j k}^{d}}
$$

para alguns valores de $d$ e $j$, considerando a norma $\left\|\psi_{j, k}(x)\right\|_{I_{j k}^{d}}$ dada pela definição (1.1.3) limitada para $x \in I_{j k}^{d}$, com espaçamento uniforme. Usamos o fato de $\psi_{j, k}(x)$ estar normalizado, e não incluímos o índice $k$ pois a norma não irá se alterar por translações. Apresentamos na tabela (2.1) os valores de $R_{j}^{d}$. Nesta tabela é possível percebermos que com o aumento dos níveis a função fica mais localizada em $I_{j k}^{d}$, e que, obviamente, valores maiores de $d$ indicam maiores valores de $R_{j}^{d}$. Em todos os casos o suporte de $\psi_{j, k}(x)$ tem concentração maior que $90 \%$ de seu suporte em $I_{j k}^{d}$, com $d=2$ este número sobe para $95 \%$, o que evidencia a propriedade de localização no espaço das ondaletas harmônicas.

\begin{tabular}{|c|cccc|}
\hline & \multicolumn{4}{|c|}{ Coeficiente de Corte $\mathbf{( d )}$} \\
\hline Nível $\mathbf{( j})$ & $\mathbf{1}$ & $\mathbf{2}$ & $\mathbf{3}$ & $\mathbf{4}$ \\
\hline 1 & 1,0000 & 1,0000 & 1,0000 & 1,0000 \\
\hline 2 & 0,9244 & 1,0000 & 1,0000 & 1,0000 \\
\hline 3 & 0,9081 & 0,9608 & 0,9837 & 1,0000 \\
\hline 4 & 0,9041 & 0,9526 & 0,9704 & 0,9802 \\
\hline 5 & 0,9031 & 0,9506 & 0,9674 & 0,9761 \\
\hline 6 & 0,9029 & 0,9501 & 0,9667 & 0,9751 \\
\hline 7 & 0,9028 & 0,9500 & 0,9665 & 0,9748 \\
\hline 8 & 0,9028 & 0,9499 & 0,9664 & 0,9748 \\
\hline 9 & 0,9028 & 0,9499 & 0,9664 & 0,9748 \\
\hline 10 & 0,9028 & 0,9499 & 0,9664 & 0,9747 \\
\hline 11 & 0,9028 & 0,9499 & 0,9664 & 0,9747 \\
\hline 12 & 0,9028 & 0,9499 & 0,9664 & 0,9747 \\
\hline 13 & 0,9028 & 0,9499 & 0,9664 & 0,9747 \\
\hline 14 & 0,9028 & 0,9499 & 0,9664 & 0,9747 \\
\hline
\end{tabular}

Tabela 2.1: Percentual do suporte de ondaletas harmônicas $\psi_{j, k}(x)$ restrito ao intervalo $I_{j k}^{d}$

Será preciso mais adiante neste trabalho analisar o suporte não somente de $\psi_{j, k}(x)$, mas também de sua derivada, $\frac{d \psi_{j, k}(x)}{d x}$, para a qual vamos nos valer da mesma limitação de suporte em $I_{j k}^{d}$. Vamos nos valer da razão:

$$
R D_{j}^{d}=\frac{\left\|\frac{d \psi_{j, k}(x)}{d x}\right\|_{I_{j k}^{d}}}{\left\|\frac{d \psi_{j, k}(x)}{d x}\right\|_{[0,1]}} .
$$

Na tabela 2.2 mostramos os percentuais de suporte para a derivada de $\psi_{j, k}(x)$. Note que há uma ligeira queda nos percentuais, mas que ainda possuem suporte predominantemente em $I_{j k}^{d}$.

Vamos estudar agora a projeção de um função no espaço de ondaletas harmônicas periodizadas. 


\begin{tabular}{|c|cccc|}
\hline & \multicolumn{4}{|c|}{ Coeficiente de Corte (d) } \\
\hline Nível ( j ) & $\mathbf{1}$ & $\mathbf{2}$ & $\mathbf{3}$ & $\mathbf{4}$ \\
\hline 1 & 1,0000 & 1,0000 & 1,0000 & 1,0000 \\
\hline 2 & 0,9177 & 1,0000 & 1,0000 & 1,0000 \\
\hline 3 & 0,9006 & 0,9577 & 0,9824 & 1,0000 \\
\hline 4 & 0,8967 & 0,9490 & 0,9682 & 0,9787 \\
\hline 5 & 0,8958 & 0,9469 & 0,9650 & 0,9743 \\
\hline 6 & 0,8956 & 0,9465 & 0,9642 & 0,9733 \\
\hline 7 & 0,8956 & 0,9463 & 0,9641 & 0,9730 \\
\hline 8 & 0,8956 & 0,9463 & 0,9640 & 0,9730 \\
\hline 9 & 0,8956 & 0,9463 & 0,9640 & 0,9729 \\
\hline 10 & 0,8957 & 0,9463 & 0,9640 & 0,9729 \\
\hline 11 & 0,8958 & 0,9464 & 0,9640 & 0,9729 \\
\hline 12 & 0,8959 & 0,9464 & 0,9640 & 0,9730 \\
\hline 13 & 0,8962 & 0,9465 & 0,9641 & 0,9730 \\
\hline 14 & 0,8968 & 0,9466 & 0,9641 & 0,9730 \\
\hline
\end{tabular}

Tabela 2.2: Percentual do suporte da derivada primeira de ondaletas harmônicas $\frac{d \psi_{j, k}(x)}{d x}$ restrito ao intervalo $I_{j k}^{d}$

Então, dada uma função $f \in L^{2}([0,1])$, podemos aproxima-la no espaço $V_{n}$, utilizando a base $B_{n}$,

$$
f(x)=a_{0}+\sum_{j=0}^{n-2} \sum_{k=0}^{2^{j}-1}\left(a_{2^{j}+k} \psi_{j, k}(x)+\tilde{a}_{2^{j}+k} \psi_{j, k}^{*}(x)\right)+a_{N / 2} \psi_{n-1}(x)
$$

onde

- $a_{0}=\langle f, 1\rangle$,

- $a_{2^{j}+k}=\left\langle f, \psi_{j, k}(x)\right\rangle$,

- $\tilde{a}_{2^{j}+k}=\left\langle f, \psi_{j, k}^{*}(x)\right\rangle$,

- $a_{N / 2}=\left\langle f, \psi_{n-1}(x)\right\rangle$.

A princípio, para $f(x)$ complexa, $a_{2^{j}+k}$ e $\tilde{a}_{2^{j}+k}$ são coeficientes distintos. Vejamos o que ocorre com $f(x)$ real, que será o caso que vamos abordar mais a diante. No caso real temos que $f=f^{*}$, então

$$
\begin{gathered}
a_{0}+\sum_{j=0}^{n-2} \sum_{k=0}^{2^{j}-1}\left(a_{2^{j}+k} \psi_{j, k}(x)+\tilde{a}_{2^{j}+k} \psi_{j, k}^{*}(x)\right)+a_{N / 2} \psi_{n-1}(x) \\
=a_{0}^{*}+\sum_{j=0}^{n-2} \sum_{k=0}^{2^{j}-1}\left(a_{2^{j}+k}^{*} \psi_{j, k}^{*}(x)+\tilde{a}_{2^{j}+k}^{*} \psi_{j, k}(x)\right)+a_{N / 2}^{*} \psi_{n-1}(x)^{*} \\
\Longleftrightarrow
\end{gathered}
$$




$$
\begin{aligned}
\tilde{a}_{2^{j}+k} & =a_{2^{j}+k}^{*}, \\
\tilde{a}_{2^{j}+k}^{*} & =a_{2^{j}+k}, \\
a_{0}^{*} & =a_{0} \\
a_{N / 2}^{*} & =a_{N / 2} .
\end{aligned}
$$

Com isso só precisamos calcular os coeficientes $\left\{a_{w}\right\}_{w=0,1, \ldots, N / 2}$, e usar a expansão

$$
f(x)=a_{0}+\sum_{j=0}^{n-2} \sum_{k=0}^{2^{j}-1}\left(a_{2^{j}+k} \psi_{j, k}(x)+a_{2^{j}+k}^{*} \psi_{j, k}^{*}(x)\right)+a_{N / 2} \psi_{n-1}(x) .
$$

David Newland desenvolveu, junto com a base de ondaletas harmônicas, um algoritmo rápido para obtenção dos coeficientes de ondaletas harmônicas, baseado em transformadas rápidas de fourier (FFT). Para deduzirmos o método, vamos usar a transformada discreta de Fourier como transformada de Fourier, para que possamos usar FFTs ao implementar numericamente o método, e o Teorema de Plancherel mostrado anteriormente na proposição (1.2.13). Consideramos a seguir os produtos internos em $\mathbb{R}^{N}$, que aproximam as integrais dos produtos em $L^{2}(\mathbb{R})$, e as aproximações dos coeficientes de ondaletas harmônicas,

$$
\begin{gathered}
a_{0}=\langle f, 1\rangle=N\langle\hat{f}, \hat{1}\rangle=\hat{f}_{0} \\
a_{N / 2}=\left\langle f, \psi_{n-1}(x)\right\rangle=N\left\langle\hat{f}, \widehat{\psi_{n-1}(x)}\right\rangle=\hat{f}_{-N / 2} \\
a_{2^{j}+k}=\left\langle f, \psi_{j, k}(x)\right\rangle=N\left\langle\hat{f}, \widehat{\psi_{j, k}(x)}\right\rangle \\
=2^{-j / 2} \sum_{w=2^{j}}^{2^{j+1}-1} \hat{f}_{w} e^{2 \pi i w \frac{k}{2^{j}}} \\
=2^{-j / 2} e^{3 \pi i k} \sum_{v=-2^{j-1}}^{2^{j-1}-1} \hat{f}_{v+3 \cdot 2^{j-1}} e^{2 \pi i v \frac{k}{2^{j}}} \\
=(-1)^{k} 2^{-j / 2} F_{k}^{-1}\left(\hat{f}_{\left[2^{j}, \ldots, 2^{j+1}-1\right]}\right) \\
=C_{j, k} F_{k}^{-1}\left(\hat{f}_{\left[2^{j}, \ldots, 2^{j+1}-1\right]}\right)
\end{gathered}
$$

onde $C_{j, k}=(-1)^{k} 2^{-j / 2}$, e $F_{k}^{-1}\left(\hat{f}_{\left[2^{j}, \ldots, 2^{j+1}-1\right]}\right)$ quer dizer a transformada discreta de Fourier inversa dos valores de $\hat{f}$ limitados ao conjunto $\left\{2^{j}, \ldots, 2^{j+1}-1\right\}$ calculado na posição de vetor $k$. Com isso temos que o vetor $a_{2^{j}+k}$, para $j$ fixado e $k$ variando, pode ser calculado usando se da transformada discreta de Fourier inversa de $\hat{f}$ restrito ao conjunto $\left\{2^{j}, \ldots, 2^{j+1}-1\right\}$. Mostramos isso em forma de diagrama na figura 2.8, neste diagrama as siglas FFT, IFFT e FHWT, querem dizer, respectivamente, transformada rápida de Fourier (Fast Fourier Transform), transformada rápida de Fourier inversa (Inverse Fast Fourier Transform) e transformada rápida de ondaletas harmônicas (Fast Harmonic Wavelet Transform). Note que as transformadas de Fourier ali presentes podem 
todas ser calculadas via transformadas rápidas de Fourier (FFTs), que é justamente o que faz deste método um método de transformada rápida.

\section{Transformada Rápida de Harmonic Wavelets (FHWT)}

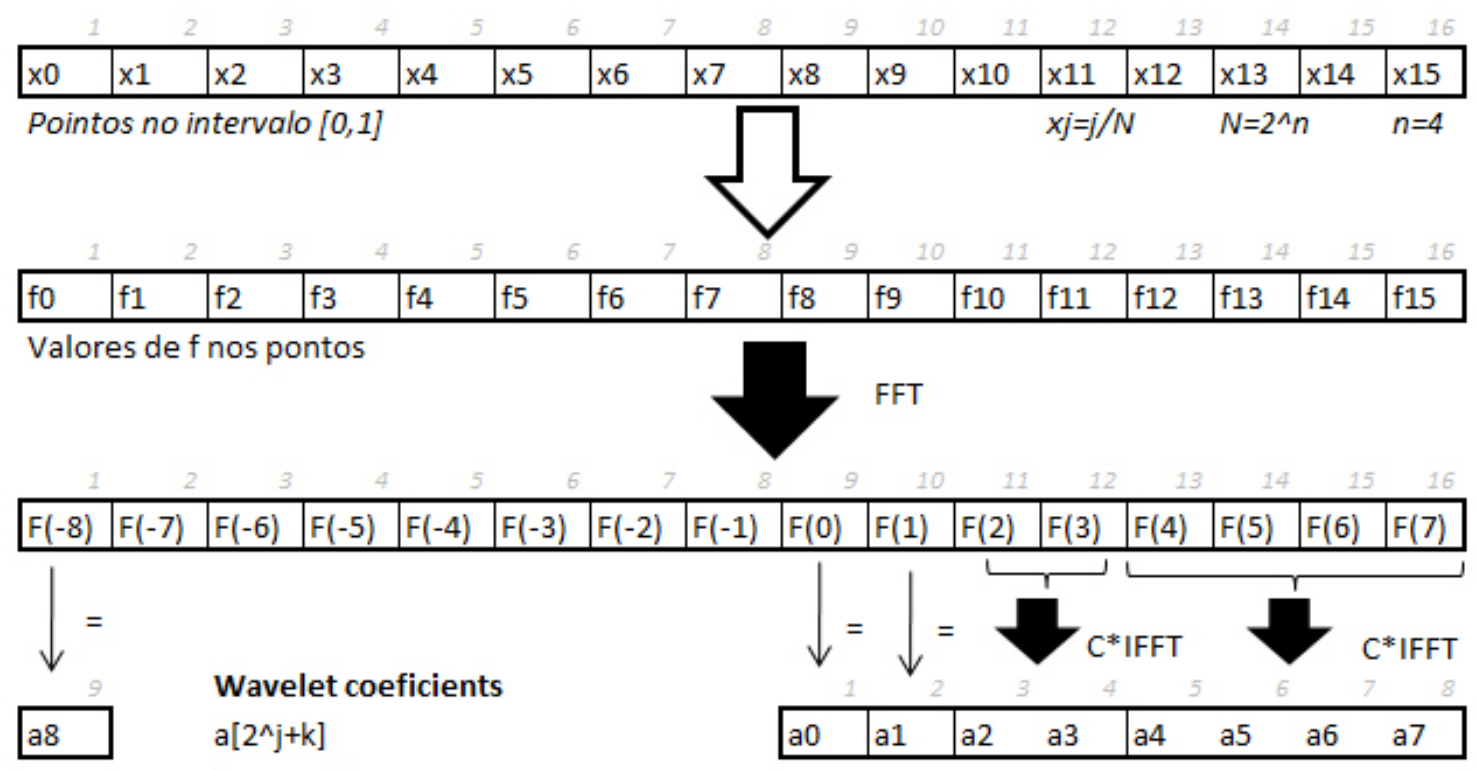

$k=0 . .2^{\wedge}(\mathrm{j})-1$

Figura 2.8: Algoritmo de transformada rápida de ondaletas harmônicas

Para ilustrar a decomposição em ondaletas harmônicas vamos considerar alguns exemplos. Utilizaremos 3 funções testes:

1. Seno

$$
f(x)=\sin (2 \pi x)
$$

2. Exponencial

$$
f(x)=e^{-\left((x-0.3)^{4}\right) 10^{5}}+1 / 2
$$

3. Pulso

$$
f(x)=(\sin (\pi(x+0.20)))^{80}
$$

Primeiro tomamos a decomposição das funções em coeficientes de ondaletas harmônicas, utilizandose do algoritmo rápido de ondaletas harmônicas, com alguns números de pontos de malha $\left(N=2^{n}\right)$. Em seguida reconstruímos as funções utilizando 10.000 pontos, uniformemente espaçados, conforme a equação 2.30. As figuras 2.9, 2.10, 2.11 mostram a função original, a aproximação obtida, o erro entre essas funções e os coeficientes de ondaletas harmônicas. Para facilitar a visualização dos 
coeficientes de ondaletas harmônicas, os mesmos são apresentados separamos por níveis, e posicionados de acordo com sua localidade no espaço. Desta forma um coeficiente $a_{j k}$ é representado por um nível $j$ e uma translação $k$, e é posicionado no intervalo sobre o valor $\frac{k}{2^{j}}$.
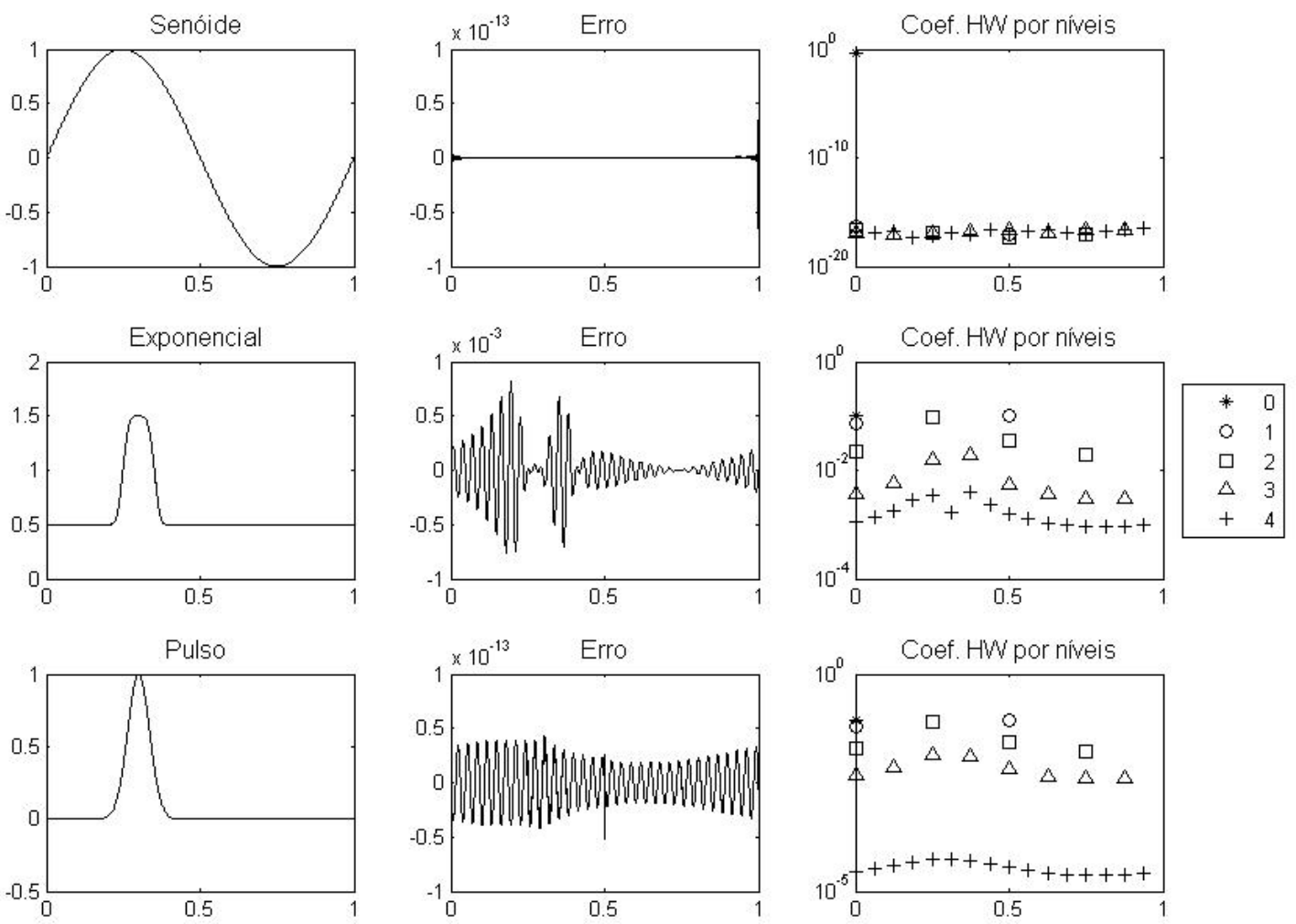

Parâmetros: $\quad N=64 \quad$ Niveis $\log 2(N)=6 \quad$ Pontos para Gráfico $=10000$

Figura 2.9: Teste para transformada de ondaleta harmônica com $n=6$. As linhas representam respectivamente as funções teste Seno, Exponencial e Pulso. Na primeira coluna são apresentados os valores da função (linha) e da aproximação por ondaletas harmônicas(tracejado), na segunda coluna a diferença entre a função teste e sua aproximação, e na terceira coluna os módulos dos coeficientes de ondaletas harmônicas por níveis em escala logarítmica. Os coeficientes de ondaletas harmônicas foram posicionados de acordo com sua localidade, isto é, um coeficiente $a_{j k}$ é colocado no valor $\frac{k}{2^{j}}$ do intervalo unitário. 

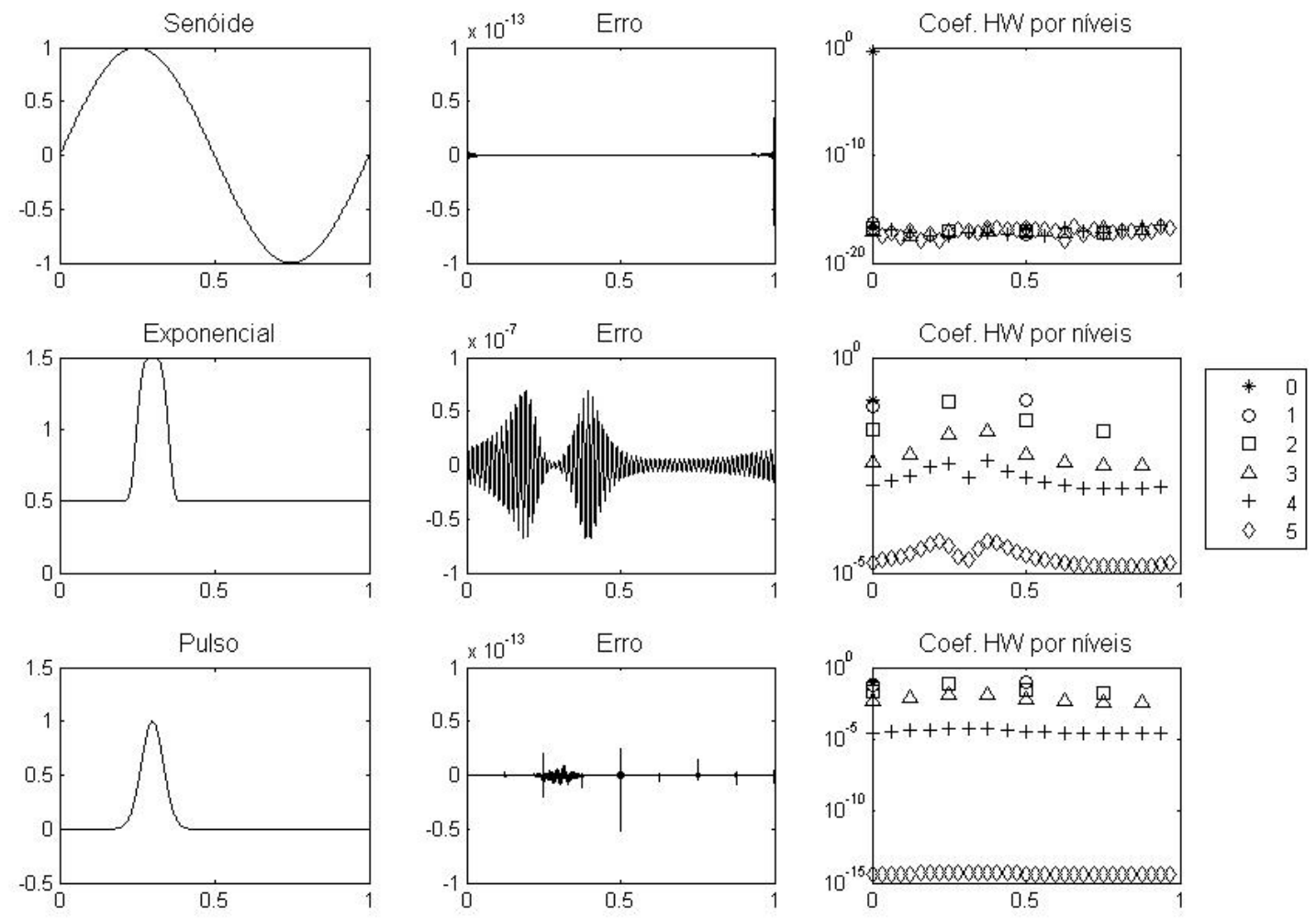

Parâmetros: $\quad N=128 \quad$ Niveis $\log 2(N)=7 \quad$ Pontos para Gráfico $=10000$

Figura 2.10: Teste para transformada de ondaleta harmônica com $n=7$. As linhas representam respectivamente as funções teste Seno, Exponencial e Pulso. Na primeira coluna são apresentados os valores da função (linha) e da aproximação por ondaletas harmônicas (tracejado), na segunda coluna a diferença entre a função teste e sua aproximação, e na terceira coluna os módulos dos coeficientes de ondaletas harmônicas por níveis em escala logarítmica. Os coeficientes de ondaletas harmônicas foram posicionados de acordo com sua localidade, isto é, um coeficiente $a_{j k}$ é colocado no valor $\frac{k}{2^{j}}$ do intervalo unitário.

\begin{tabular}{|c|ccc|}
\hline $\mathrm{n}$ & Seno & Exponencial & Pulso \\
\hline 3 & $6,481 \mathrm{E}-14$ & $6,678 \mathrm{E}-01$ & $6,729 \mathrm{E}-01$ \\
4 & $6,481 \mathrm{E}-14$ & $1,661 \mathrm{E}-01$ & $1,232 \mathrm{E}-01$ \\
5 & $6,481 \mathrm{E}-14$ & $5,091 \mathrm{E}-02$ & $6,221 \mathrm{E}-04$ \\
6 & $6,481 \mathrm{E}-14$ & $8,533 \mathrm{E}-04$ & $5,767 \mathrm{E}-14$ \\
7 & $6,481 \mathrm{E}-14$ & $6,898 \mathrm{E}-08$ & $5,167 \mathrm{E}-14$ \\
8 & $6,487 \mathrm{E}-14$ & $5,784 \mathrm{E}-14$ & $5,172 \mathrm{E}-14$ \\
9 & $6,480 \mathrm{E}-14$ & $5,795 \mathrm{E}-14$ & $5,166 \mathrm{E}-14$ \\
10 & $6,496 \mathrm{E}-14$ & $5,795 \mathrm{E}-14$ & $5,165 \mathrm{E}-14$ \\
\hline
\end{tabular}

Tabela 2.3: Erros associados à transformada de ondaletas harmônicas 

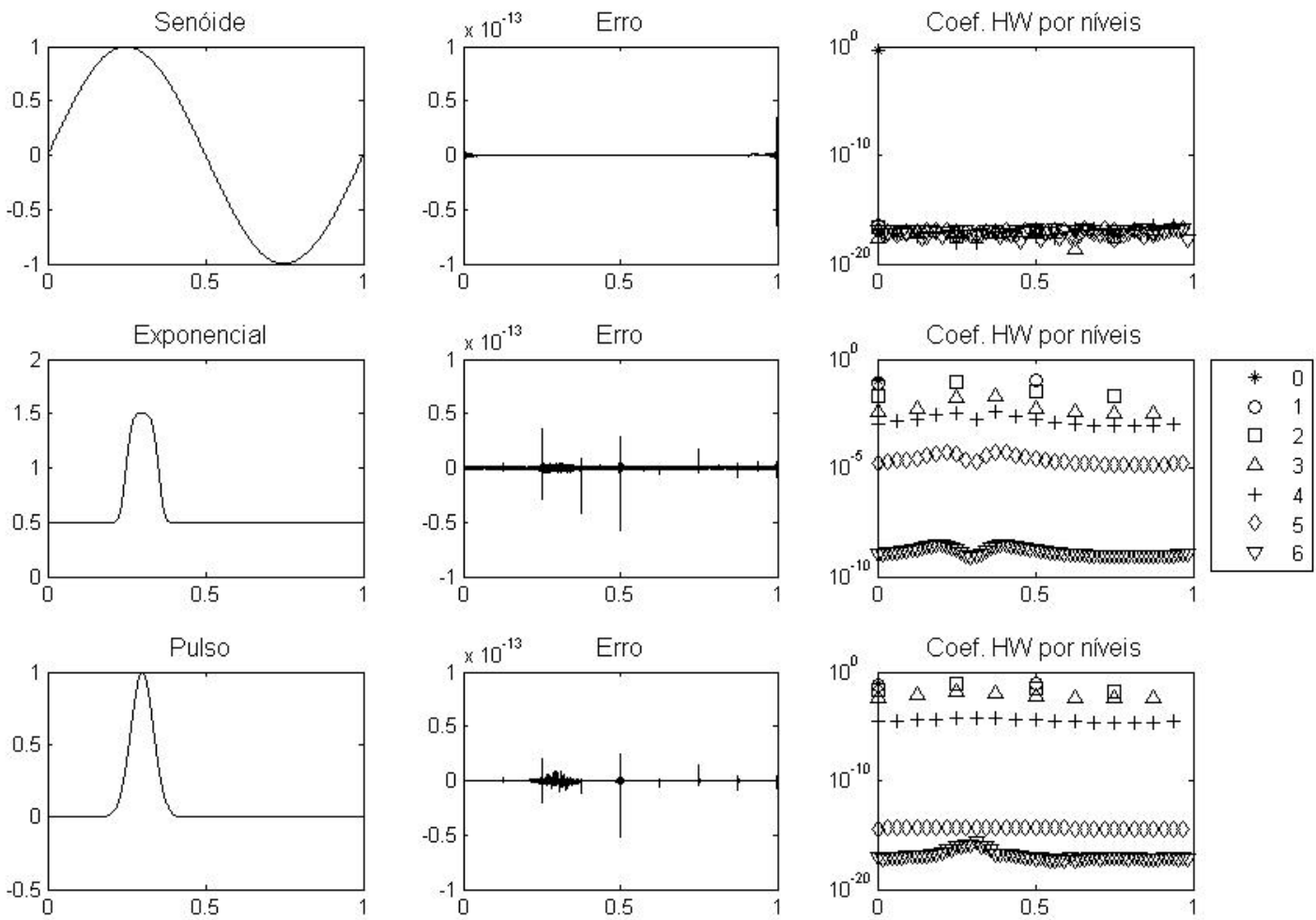

Parâmetros: $\quad N=256 \quad$ Niveis $\log 2(N)=8$ Pontos para Gráfico $=10000$

Figura 2.11: Teste para transformada de ondaleta harmônica com $n=8$. As linhas representam respectivamente as funções teste Seno, Exponencial e Pulso. Na primeira coluna são apresentados os valores da função (linha) e da aproximação por ondaletas harmônicas (tracejado), na segunda coluna a diferença entre a função teste e sua aproximação, e na terceira coluna os módulos dos coeficientes de ondaletas harmônicas por níveis em escala logarítmica. Os coeficientes de ondaletas harmônicas foram posicionados de acordo com sua localidade, isto é, um coeficiente $a_{j k}$ é colocado no valor $\frac{k}{2^{j}}$ do intervalo unitário. 


\section{Capítulo 3}

\section{Método de Galerkin}

Problemas ligados a equações diferenciais nem sempre têm soluções analíticas, por isso muitas vezes tomam-se aproximações da solução. Existem diversos métodos para se aproximar soluções de problemas de equações diferenciais. Neste capítulo abordamos o método de Galerkin, e seu caso particular, o método Galerkin-Ondaleta.

\subsection{Fundamentos}

Considere uma equação diferencial, linear ou não, dada por

$$
D u=f
$$

onde $D: X \rightarrow Y$ é o operador diferencial entre os espaços de funções $X$ e $Y$, e $u \in X$. Assumiremos que o problema tem solução única e que o problema está bem posto. Vamos considerar $X$ um espaço com produto interno com base ortonormal $\left\{x_{1}, x_{2}, \ldots\right\}$, e $Y$ um espaço com produto interno com base ortonormal $\left\{y_{1}, y_{2}, \ldots\right\}$. Podemos então olhar para equação 3.1 na seguinte forma:

$$
\left\langle D u, y_{j}\right\rangle_{Y}=\left\langle f, y_{j}\right\rangle_{Y}, \quad j \in \mathbb{N}
$$

Agora seja

$$
X_{N}=\operatorname{span}\left\{x_{k}\right\}_{k=1,2, \ldots, N}
$$

o espaço vetorial de dimensão finita gerado pela base de funções $x_{k}$, que aproximam o espaço $X$ quando N vai para infinito. Com isso podemos buscar uma solução aproximada de (3.1), que denominaremos por $\tilde{u}$, dada por

$$
\tilde{u}=\sum_{k=1}^{N} \alpha_{k} x_{k}
$$

e ainda considerar o resíduo,

$$
r=u-\tilde{u}
$$

Para uma aproximação de solução deste problema resolvemos um sistema da forma

$$
\left\langle D \tilde{u}, y_{j}\right\rangle_{Y}=\left\langle f, y_{j}\right\rangle_{Y}, \quad j=1,2, \ldots, N
$$


Ou podemos pensar, de forma análoga, que a projeção do resíduo se anula,

$$
\left\langle D r, y_{j}\right\rangle_{Y}=0, \quad j=1,2, \ldots, N
$$

No caso de $D$ ser um operador linear, que denominaremos por $L$, chegamos a um sistema linear

$$
\begin{aligned}
\left\langle L \tilde{u}, y_{j}\right\rangle_{Y_{N}} & = \\
& =\sum_{k=1}^{N} \alpha_{k}\left\langle L\left(x_{k}\right), y_{j}\right\rangle_{Y_{N}} \\
& =\left\langle f, y_{j}\right\rangle_{Y_{N}}, \quad j=1,2, \ldots, N .
\end{aligned}
$$

Neste caso denominamos os produtos internos $\left\langle L\left(x_{k}\right), y_{j}\right\rangle$ por coeficientes de conexão lineares, que se forem denotados por $a_{j k}$, e sendo $A$ a matriz formada por estes coeficientes, com $b$ um vetor com os elementos $b_{j}=\left\langle f, y_{j}\right\rangle_{Y_{N}}$, geram um sistema linear $A \alpha=b$. Após resolver esta sistema, utilizam-se os valores obtidos de $\alpha$ para gerar $\tilde{u}$, chegando portanto a uma aproximação numérica da solução exata $u$. Este método é conhecido por método de Galerkin.

No método de Galerkin é desejável que tenhamos uma matriz $A$ que seja esparsa e que tenha número de condição pequeno (definição 1.1.6). Isso reduz o custo computacional do método e reduz o erro na aproximação da solução.

Para problemas definidos em intervalos fechados de $\mathbb{R}$, como será nosso caso, por exemplo [0,1], o método de Galerkin usa uma base cujas componentes naturalmente satisfazem as condições de fronteira. No nosso caso vamos trabalhar com fronteiras periódicas em [0,1]. Portanto, adotaremos bases com funções periódicas com período 1.

O método Galerkin-Ondaleta é um caso particular do Método de Galerkin no qual utilizamos como base para aproximação da solução uma base de ondaletas.

\subsection{Base de Fourier}

O desenvolvimento desta parte pode ser encontrada nos livros de Canuto et al. (1988) ou Peyret (2000). Destacaremos partes que serão usadas no estudo mais adiante.

Buscamos uma solução para um problema de equações diferenciais, como em (3.1), com $X=Y$ espaços de funções periódicas em $L^{2}([0,1])$. Aproximamos tanto $X$ como $Y$ por $S_{N}$, onde $S_{N}$ é espaço gerado pelos polinômios trigonométricos com $N$ elementos distintos e números de onda entre $-N / 2$ e $N / 2-1$, escrito como

$$
S_{N}=\operatorname{span}\left\{e^{2 \pi i w x}\right\}_{w=-N / 2, \ldots, N / 2-1} .
$$

Vamos tomar uma aproximação da solução, $u_{N}$, definida em $S_{N}$, que pode ser escrita como,

$$
u_{N}(x)=\sum_{w=-N / 2}^{N / 2-1} a_{w} e^{2 \pi i w x}
$$


onde,

$$
a_{w}=\left\langle u_{N}(x), e^{2 \pi i w x}\right\rangle=\int_{0}^{1} u_{N}(x) e^{-2 \pi i w x} d x .
$$

\subsubsection{Projeção de derivadas}

Se tomarmos a $l$-ésima derivada de $u_{N}(x)$,

$$
\frac{d^{l} u_{N}(x)}{d x^{l}}=(2 \pi i)^{l} \sum_{w=-N / 2}^{N / 2-1} w^{l} a_{w} e^{2 \pi i w x}
$$

e projetarmos nas direções dos elementos da base $\left\{e^{2 \pi i \lambda x}\right\}_{\lambda=-N / 2, \ldots, N / 2-1}$, teremos,

$$
\left\langle\frac{d^{l} u_{N}(x)}{d x^{l}}, e^{2 \pi i \lambda x}\right\rangle=(2 \pi i)^{l}(\lambda)^{l} a_{\lambda}
$$

\subsubsection{Projeção de não linearidades com derivadas}

Tomaremos agora $v$ uma função escrita na base $S_{N}$, e $u_{x}=\frac{d u}{d x}$ como acima. Então teremos que

$$
v u_{x}=2 \pi i \sum_{k=-N / 2}^{N / 2-1} a_{k} e^{2 \pi i k x} \sum_{w=-N / 2}^{N / 2-1} w b_{w} e^{2 \pi i w x}=2 \pi i \sum_{k=-N / 2}^{N / 2-1} \sum_{w=-N / 2}^{N / 2-1} w a_{k} b_{w} e^{2 \pi i(k+w) x}
$$

onde $a_{k}$ indicam coeficientes de $v$, e $b_{w}$ de $u$.

Se projetarmos $v u_{x}$ vamos ter,

$$
\begin{aligned}
\left\langle v u_{x}, e^{2 \pi i \lambda x}\right\rangle & =2 \pi i \sum_{k=-N / 2}^{N / 2-1} \sum_{w=-N / 2}^{N / 2-1} w a_{k} b_{w} \int_{0}^{1} e^{2 \pi i(k+w-\lambda) x} d x \\
& =2 \pi i \sum_{k=-N / 2}^{N / 2-1} \sum_{w=-N / 2}^{N / 2-1} w a_{k} b_{w} \delta_{k+w, \lambda} \\
& =2 \pi i \sum_{\substack{k+w=\lambda \\
-\frac{N}{2} \leq k, w \leq \frac{N}{2}-1}} w a_{k} b_{w}, \quad-N / 2 \leq \lambda \leq N / 2-1 .
\end{aligned}
$$

O cálculo do produto de convolução $\sum_{k+w=\lambda} w a_{k} b_{w}$, para $\lambda=-N / 2 \leq \lambda \leq N / 2-1$, requer da ordem de $N^{2}$ operações. Uma alternativa mais eficiente é utilizar uma abordagem pseudo-espectral, onde resolve-se esse produto no domínio do espaço. Esta outra abordagem faz com que o custo do cálculo do produto de convolução seja da ordem de $N \log N$. Vejamos como funciona.

Para vermos de uma forma mais geral, considere $f(x)=v(x), g(x)=u_{x}(x)$ e $h(x)=f(x) g(x)$. Então temos que,

$$
f(x)=\sum_{k=-\infty}^{+\infty} \hat{f}_{k} e^{2 \pi i k x}
$$




$$
\begin{gathered}
g(x)=\sum_{w=-\infty}^{+\infty} \hat{g}_{w} e^{2 \pi i w x}, \\
\hat{h}_{\lambda}=\int_{0}^{1} h(x) e^{-2 \pi i \lambda x} d x,
\end{gathered}
$$

e o resultante produto de convolução para o $[0,1]$,

$$
\hat{h}_{\lambda}=\sum_{k+w=\lambda} \hat{f}_{k} \hat{g}_{w}, \quad \lambda \in \mathbb{Z}
$$

Por outro lado, quando consideramos as séries de Fourier de $f$ e $g$ truncadas em números onda entre $-\frac{N}{2}, \ldots, \frac{N}{2}-1$, temos o produto de convolução dado por,

$$
\hat{h}_{\lambda}=\sum_{\substack{k+w=\lambda \\-\frac{N}{2} \leq k, w \leq \frac{N}{2}-1}} \hat{f}_{k} \hat{g}_{w}, \quad-N / 2 \leq \lambda \leq N / 2-1,
$$

que é o que precisamos obter. Por outro lado na malha temos que

$$
\begin{aligned}
F_{l} & =\sum_{k=-N / 2}^{N / 2-1} \hat{f}_{k} e^{2 \pi i k x_{l}}, \quad l=0,1, \ldots, N-1, \\
G_{l} & =\sum_{w=-N / 2}^{N / 2-1} \hat{g}_{w} e^{2 \pi i w x_{l}}, \quad l=0,1, \ldots, N-1,
\end{aligned}
$$

e tomando $H_{l}=F_{l} \cdot G_{l}, l=0,1, \ldots, N-1$, teremos,

$$
\hat{H}_{\lambda}=\sum_{\substack{k+w=\lambda \\-\frac{N}{2} \leq k, w \leq \frac{N}{2}-1}} \hat{f}_{k} \hat{g}_{w}+\sum_{\substack{k+w=\lambda \pm N \\-\frac{N}{2} \leq k, w \leq \frac{N}{2}-1}} \hat{f}_{k} \hat{g}_{w}, \quad-N / 2 \leq \lambda \leq N / 2-1 .
$$

O método pseudo-espectral consiste em transformar os coeficientes $\hat{f}$ e $\hat{g}$, utilizando a transformada discreta inversa de Fourier, nos valores das funções na malha, $F_{l}$ e $G_{l}$. Com estes valores calculamos $H_{l}=F_{l} G_{l}$, e tomamos a transformada discreta de Fourier de $H_{l}$, chegando em $\hat{H}$, dado pela equação (3.23). Este algoritmo pode ser calculado com transformas rápidas de Fourier (FFT), resultando em $O\left(N \log _{2} N\right)$ operações. Agora note na equação 3.23 que temos 2 elementos do lado direito da equação. O primeiro é aquele que gostaríamos de calcular, o outro é o erro devido ao "aliasing", indesejado. Uma forma de remover este termo indesejado é tomando $M=3 N / 2$ pontos de malha, e zerar os coeficientes de fourier de frequências mais altas, menores que $-N / 2 \mathrm{e}$ maiores que $N / 2-1$. Com isso preservamos a ordem do número de operações, $O\left(N \log _{2}(N)\right)$.

No nosso caso devemos transformar os coeficientes de Fourier $a_{k}$ e $w b_{w}$ em valores da malha numérica, efetuar a multiplicação, e depois realizar uma transformada discreta de Fourier para voltar ao domínio espectral, finalizando o cálculo do produto de convolução. Mais detalhes sobre 
métodos pseudo-espectrais podem ser encontrados nas diversas referências sobre métodos espectrais para dinâmica de fluidos, destacamos o livro de Canuto et al. (1988) para detalhes.

\subsubsection{Problema Teste}

Vamos considerar um problema teste que utilize as projeções definidas anteriormente para ilustrar o método de Galerkin utilizando a base de Fourier.

Considere o problema de valor inicial,

$$
\begin{cases}D(u(x, t))=\frac{\partial u(x, t)}{\partial t}+c \frac{\partial u(x, t)}{\partial x}+v(x, t) \frac{\partial u(x, t)}{\partial x}-\nu \frac{\partial^{2} u(x, t)}{\partial x^{2}}=0, & x \in(0,1), \\ u(x, 0)=f(x), & x \in[0,1], \\ u(0, t)=u(1, t), & t \geq 0,\end{cases}
$$

onde $f(x) \in L^{2}([0,1])$ e , $v(x, t) \in L^{2}([0,1])$ na variável $x$, para cada $t$ fixado. Consideramos ainda a possibilidade de tomarmos $v=u$. Buscamos uma aproximação para a solução exata $u(x, t)$, para tanto vamos definir $u_{N}(x, t)$ utilizando separação de variáveis e expansão em série de Fourier,

$$
u_{N}(x, t)=\sum_{k=-N / 2}^{N / 2-1} a_{k}(t) e^{2 \pi i k x}
$$

e expansão em série de Fourier de $f$ e $v$,

$$
\begin{gathered}
f_{N}(x)=\sum_{k=-N / 2}^{N / 2-1} b_{k} e^{2 \pi i k x}, \\
v_{N}(x, t)=\sum_{w=-N / 2}^{N / 2-1} d_{w}(t) e^{2 \pi i w x} .
\end{gathered}
$$

Pelo método de Galerkin temos que resolver um sistema não linear da forma,

$$
\left\langle\tilde{D} u_{N}, e^{2 \pi i \lambda x}\right\rangle_{L^{2}([0,1])}=0, \quad \lambda=-N / 2, \ldots, N / 2-1,
$$

onde $\tilde{D} u_{N}$ pode ser escrito como,

$$
\begin{aligned}
\tilde{D} u_{N}(x, t) & =\sum_{k=-N / 2}^{N / 2-1}\left(\frac{\partial a_{k}(t)}{\partial t}+c(2 \pi i k) a_{k}(t)\right) e^{2 \pi i k x} \\
& +\sum_{k=-N / 2}^{N / 2-1} \sum_{w=-N / 2}^{N / 2-1}\left(d_{w}(t) e^{2 \pi i w x}(2 \pi i k) a_{k}(t) e^{2 \pi i k x}\right) \\
& -\nu \sum_{k=-N / 2}^{N / 2-1}\left((2 \pi i k)^{2} a_{k}(t) e^{2 \pi i k x}\right),
\end{aligned}
$$


e é uma aproximação do operador $D$. Para evitar notações excessivas seguiremos usando o operador $D$, sem o símbolo de aproximação, para designar o operador aproximado. Assim teremos,

$$
\begin{aligned}
\left\langle D u_{N}, e^{2 \pi i \lambda x}\right\rangle_{L^{2}([0,1])} & =\frac{\partial a_{\lambda}(t)}{\partial t}+c(2 \pi i \lambda) a_{\lambda}(t) \\
& +\sum_{k+w=\lambda}\left((2 \pi i k) d_{w}(t) a_{k}(t)\right) \\
& -\nu\left((2 \pi i \lambda)^{2} a_{\lambda}(t)\right) \\
& =0 .
\end{aligned}
$$

Portanto ficamos com um sistema não linear de EDOs a ser resolvido, dado por,

$$
\left\{\begin{array}{l}
\frac{\partial a_{\lambda}(t)}{\partial t}=-2 \pi i \lambda c a_{\lambda}(t)-\sum_{k+w=\lambda}\left(2 \pi i k d_{w}(t) a_{k}(t)\right)+\nu(2 \pi i \lambda)^{2} a_{\lambda}(t) \\
a_{\lambda}(0)=b_{\lambda}, \quad \lambda=-N / 2, \ldots, N / 2-1
\end{array} .\right.
$$

Neste sistema, o conjunto de valores $b_{\lambda}$ pode ser obtido via transformadas rápidas de Fourier (FFTs) de $f$, e após resolver o sistema, utiliza-se uma inversa de FFT para transformar os coeficientes $a_{\lambda}$ em $u_{N}$, que é a solução aproximada. Para resolver este sistema diversos métodos numéricos para EDOs podem ser usados, discutiremos mais a fundo isso nos testes numéricos.

\subsection{Base de ondaletas harmônicas}

Visamos novamente obter uma aproximação da solução de um problema de equações diferenciais, como em (3.1), com $X=Y=L^{2}([0,1])$, que possa ser escrito na base de ondaletas harmônicas. Para tanto aproximamos tanto $X$ como $Y$ por $B_{n}$, que é o espaço vetorial gerado pela base de ondaletas harmônicas para o $L^{2}([0,1])$,

$$
B_{n}=\operatorname{span}\left\{\phi, \psi_{j k}, \psi_{j k}^{*}, \psi_{n-1}\right\}_{j=0,1, \ldots, n-2 ; \quad k=0,1, \ldots, 2^{j}-1},
$$

e a aproximação $u_{N}(x)$ será dada por

$$
u_{N}(x)=a_{0}+\sum_{j=0}^{n-2} \sum_{k=0}^{2^{j}-1}\left(a_{2^{j}+k} \psi_{j, k}(x)+a_{2^{j}+k}^{*} \psi_{j, k}^{*}(x)\right)+a_{N / 2} \psi_{n-1}(x) .
$$

Vamos analisar alguns casos possíveis de operadores diferenciais atuando no espaço espectral de coeficientes de ondaletas harmônicas. 


\subsubsection{Projeção de derivadas primeiras}

Considerando a função $u$ escrita na base de ondaletas harmônicas, dada na equação (3.33), vamos inicialmente olhar quem é $\frac{d u}{d x}=u_{x}$.

$$
u_{x}=\frac{d u(x)}{d x}=\sum_{j=0}^{n-2} \sum_{k=0}^{2^{j}-1}\left(a_{2^{j}+k} \frac{d \psi_{j, k}(x)}{d x}+a_{2^{j}+k}^{*} \frac{d \psi_{j, k}^{*}(x)}{d x}\right)+a_{N / 2} \frac{d \psi_{n-1}(x)}{d x}
$$

Onde

$$
\frac{d \psi_{j, k}(x)}{d x}=2 \pi i 2^{-j / 2} \sum_{m_{j}=2^{j}}^{2^{j+1}-1} m_{j} e^{2 \pi i m_{j}\left(x-\frac{k}{2^{j}}\right)}, \quad j=0,1, . ., n-2, \quad k=0,1, \ldots, 2^{j}-1
$$

$\mathrm{e}$

$$
\frac{d \psi_{n-1}(x)}{d x}=-i N \pi e^{-i N \pi x}
$$

Vamos olhar para a transformada de Fourier das derivadas das ondaletas harmônicas. Omitiremos os cálculos, mas destacamos que usamos somente as definições e as propriedades da transformada de Fourier, de forma análoga ao realizado na equação (2.16), para chegarmos em

$$
\left(\frac{d \widehat{\psi_{j, k}(x)}}{d x}\right)_{w}= \begin{cases}2 \pi i w 2^{-j / 2} e^{-2 \pi i w \frac{k}{2^{j}}} & \text { quando } w=2^{j}, \ldots, 2^{j+1}-1 \\ 0 & \text { caso contrário }\end{cases}
$$

$\mathrm{e}$

$$
\left(\frac{\left.d \widehat{\psi_{n-1}(} x\right)}{d x}\right)_{w}=-N \pi i, \quad w=-N / 2 .
$$

Note que preservamos a localidade do suporte no domínio de frequências. Vamos estimar agora os coeficientes de conexão pertinentes. Verifica-se usando o Teorema de Plancherel (1.2.13) que os produtos internos das derivadas das ondaletas harmônicas em relação aos elementos da base são nulos se forem de níveis distintos, pois não apresentam intersecção de suporte no domínio de frequências. Ou seja, os produtos

- $\left\langle\frac{d \psi_{j, k}(x)}{d x}, \phi(x)\right\rangle$,

- $\left\langle\frac{d \psi_{j, k}(x)}{d x}, \psi_{p, q}(x)\right\rangle \operatorname{com} p \neq j$,

- $\left\langle\frac{d \psi_{j, k}(x)}{d x}, \psi_{p, q}^{*}(x)\right\rangle$,

- $\left\langle\frac{d \psi_{j, k}(x)}{d x}, \psi_{n-1}(x)\right\rangle$,

- $\left\langle\frac{d \psi_{j, k}^{*}(x)}{d x}, \phi(x)\right\rangle$,

- $\left\langle\frac{d \psi_{j, k}^{*}(x)}{d x}, \psi_{p, q}^{*}(x)\right\rangle \operatorname{com} p \neq j$, 
- $\left\langle\frac{d \psi_{j, k}^{*}(x)}{d x}, \psi_{p, q}(x)\right\rangle$,

- $\left\langle\frac{d \psi_{j, k}^{*}(x)}{d x}, \psi_{n-1}(x)\right\rangle$,

- $\left\langle\frac{d \psi_{n-1}(x)}{d x}, \phi(x)\right\rangle$,

- $\left\langle\frac{d \psi_{n-1}(x)}{d x}, \psi_{p, q}^{*}(x)\right\rangle$,

- $\left\langle\frac{d \psi_{n-1}(x)}{d x}, \psi_{p, q}(x)\right\rangle$,

são todos nulos. Ficamos então com os seguintes produtos internos:

$$
\begin{aligned}
\left\langle\frac{d \psi_{n-1}(x)}{d x}, \psi_{n-1}(x)\right\rangle & =\int_{0}^{1}-i N \pi e^{-i N \pi x} e^{i N \pi x} d x \\
& =-N \pi i
\end{aligned}
$$

Aqui valem duas observações. A primeira é que o coeficiente $\gamma_{k q}^{j}$ pode ser calculado via FFT. A segunda é o fato interessante do coeficiente só depender da distância entre os elementos de translação. Isso resulta em uma propriedade útil para implementação numérica,

$$
-\left(\gamma_{q k}^{j}\right)^{*}=\gamma_{k q}^{j}
$$

pois desta forma precisamos armazenar os elementos cujas distâncias são tais que $k \geq q$, e usarmos a propriedade quando $q>k$.

Ao projetarmos portanto $u_{x}$ na base de ondaleta harmônicas vamos obter:

$$
\begin{aligned}
\left\langle u_{x}, \phi\right\rangle & =0, \\
\left\langle u_{x}, \psi_{r, s}(x)\right\rangle & =\sum_{k=0}^{2^{r}-1} a_{2^{r}+k} \gamma_{k s}^{r}, \\
\left\langle u_{x}, \psi_{n-1}(x)\right\rangle & =-N \pi i a_{N / 2} .
\end{aligned}
$$


Onde $r=0,1, \ldots, n-2$ e $s=0,1, \ldots, 2^{r}-1$. Se denominarmos por

$$
\begin{aligned}
c_{0} & =\left\langle u_{x}, \phi\right\rangle, \\
c_{2^{r}+s} & =\left\langle u_{x}, \psi_{r, s}(x)\right\rangle, \\
c_{N / 2} & =\left\langle u_{x}, \psi_{n-1}(x)\right\rangle,
\end{aligned}
$$

e tomarmos o vetor $\vec{c}$, cujas entradas são os elementos $c_{k}$ para $k=0,1, \ldots, N / 2$, e o vetor $\vec{a}$, cujas entradas são os elementos $a_{k}$ para $k=0,1, \ldots, N / 2$, então valerá que,

$$
\vec{c}=M \vec{a},
$$

onde $M$ é uma matrix esparsa com entradas dadas pelos coeficientes de conexão lineares de 1a. ordem. A estrutura de esparsidade de $M$ pode ser representada pela matriz

$$
\left[\begin{array}{cccccccccc}
0 & 0 & 0 & 0 & 0 & 0 & 0 & 0 & \cdots & 0 \\
0 & \star & 0 & 0 & 0 & 0 & 0 & 0 & \cdots & 0 \\
0 & 0 & \star & \star & 0 & 0 & 0 & 0 & \cdots & 0 \\
0 & 0 & \star & \star & 0 & 0 & 0 & 0 & \cdots & 0 \\
0 & 0 & 0 & 0 & \star & \star & \star & \star & \cdots & 0 \\
0 & 0 & 0 & 0 & \star & \star & \star & \star & \cdots & 0 \\
0 & 0 & 0 & 0 & \star & \star & \star & \star & \cdots & 0 \\
0 & 0 & 0 & 0 & \star & \star & \star & \star & \cdots & 0 \\
\vdots & \vdots & \vdots & \vdots & \vdots & \vdots & \vdots & \vdots & \ddots & 0 \\
0 & 0 & 0 & 0 & 0 & 0 & 0 & 0 & 0 & \star
\end{array}\right]
$$

onde o símbolo * indica elemento não nulo.

O custo computacional de se avaliar a projeção de $u_{x}$ na base de ondaletas harmônicas é basicamente determinado pela expressão dada na equação 3.43. Nela, para cada nível $r$, e escala $s$, temos $2^{r}$ operações, chegando a um máximo de $2^{n-2}=N / 4$ operações, quando $r=n-2$, ou seja, temos $O(N)$ operações para cada par $(r, s)$. Isso gera um custo computacional da ordem de $O\left(N^{2}\right)$ para se avaliar o conjunto completo da projeção de $u_{x}$ na base de ondaleta harmônicas, pois temos exatamente $N / 2-1$ pares da forma $(r, s)$. Até aqui nada foi usado em especial de se tratar de uma base de ondaletas, portanto vamos considerar agora a propriedade de localidade do suporte das ondaletas harmônicas, dada na equação 2.25 . Portanto, se tomarmos um parâmetro $d$ para forçar uma delimitação de suporte, o produto $\left\langle\frac{d \psi_{j, k}(x)}{d x}, \psi_{j, s}(x)\right\rangle$ será considerado nulo se $d<|k-s|<2^{j}-d$, resultado em uma simplificação da equação 3.43,

$$
\left\langle u_{x}, \psi_{r, s}(x)\right\rangle=\sum_{k=\bmod \left(s-d, 2^{r}\right)}^{\bmod \left(s+d, 2^{r}\right)} a_{2^{r}+k} \gamma_{k s}^{r},
$$

onde, o somatório deve ser entendido como circular, ou em aritmética modular $(\bmod )$. Isso torna 
o número de operações no máximo $2 d+1$ por par $(r, s)$, e o algoritmo para se avaliar o conjunto completo da projeção de $u_{x}$ na base de ondaleta harmônicas passa a ter $O(N)$ de custo computacional. Note que a restrição de suporte foi utilizada apenas para definir quais produtos internos serão desprezados, mas no cálculo do coeficiente de conexão $\gamma_{k s}^{r}$ é utilizado a ondaleta completa, sobre todo o intervalo. A estrutura de esparsidade da matriz $M$ utilizando a propriedade de suporte localizado da base fica sendo, com $d=1$ por exemplo,

$$
\left[\begin{array}{cccccccccccccccccc}
0 & 0 & 0 & 0 & 0 & 0 & 0 & 0 & 0 & 0 & 0 & 0 & 0 & 0 & 0 & 0 & \cdots & 0 \\
0 & \star & 0 & 0 & 0 & 0 & 0 & 0 & 0 & 0 & 0 & 0 & 0 & 0 & 0 & 0 & \cdots & 0 \\
0 & 0 & \star & \star & 0 & 0 & 0 & 0 & 0 & 0 & 0 & 0 & 0 & 0 & 0 & 0 & \cdots & 0 \\
0 & 0 & \star & \star & 0 & 0 & 0 & 0 & 0 & 0 & 0 & 0 & 0 & 0 & 0 & 0 & \cdots & 0 \\
0 & 0 & 0 & 0 & \star & \star & 0 & \star & 0 & 0 & 0 & 0 & 0 & 0 & 0 & 0 & \cdots & 0 \\
0 & 0 & 0 & 0 & \star & \star & \star & 0 & 0 & 0 & 0 & 0 & 0 & 0 & 0 & 0 & \cdots & 0 \\
0 & 0 & 0 & 0 & 0 & \star & \star & \star & 0 & 0 & 0 & 0 & 0 & 0 & 0 & 0 & \cdots & 0 \\
0 & 0 & 0 & 0 & \star & 0 & \star & \star & 0 & 0 & 0 & 0 & 0 & 0 & 0 & 0 & \cdots & 0 \\
0 & 0 & 0 & 0 & 0 & 0 & 0 & 0 & \star & \star & 0 & 0 & 0 & 0 & 0 & \star & \cdots & 0 \\
0 & 0 & 0 & 0 & 0 & 0 & 0 & 0 & \star & \star & \star & 0 & 0 & 0 & 0 & 0 & \cdots & 0 \\
0 & 0 & 0 & 0 & 0 & 0 & 0 & 0 & 0 & \star & \star & \star & 0 & 0 & 0 & 0 & \cdots & 0 \\
0 & 0 & 0 & 0 & 0 & 0 & 0 & 0 & 0 & 0 & \star & \star & \star & 0 & 0 & 0 & \cdots & 0 \\
0 & 0 & 0 & 0 & 0 & 0 & 0 & 0 & 0 & 0 & 0 & \star & \star & \star & 0 & 0 & \cdots & 0 \\
0 & 0 & 0 & 0 & 0 & 0 & 0 & 0 & 0 & 0 & 0 & 0 & \star & \star & \star & 0 & \cdots & 0 \\
0 & 0 & 0 & 0 & 0 & 0 & 0 & 0 & 0 & 0 & 0 & 0 & 0 & \star & \star & \star & \cdots & 0 \\
0 & 0 & 0 & 0 & 0 & 0 & 0 & 0 & \star & 0 & 0 & 0 & 0 & 0 & \star & \star & \cdots & 0 \\
\vdots & \vdots & \vdots & \vdots & \vdots & \vdots & \vdots & \vdots & \vdots & \vdots & \vdots & \vdots & \vdots & \vdots & \vdots & \vdots & \ddots & 0 \\
0 & 0 & 0 & 0 & 0 & 0 & 0 & 0 & 0 & 0 & 0 & 0 & 0 & 0 & 0 & 0 & 0 & \star
\end{array}\right]
$$

\subsubsection{Projeção de derivadas segundas}

As estimativas para $u_{x x}$ são essencialmente idênticas às realizadas para $u_{x}$, então vamos ser mais breves nesta seção. Novamente considerando a função $u$ escrita na base de ondaletas harmônicas, dada na equação 3.33, vamos olhar quem é $\frac{d^{2} u}{d x^{2}}=u_{x x}$.

$$
u_{x x}:=\frac{d^{2} u(x)}{d x^{2}}=\sum_{j=0}^{n-2} \sum_{k=0}^{2^{j}-1}\left(a_{2^{j}+k} \frac{d^{2} \psi_{j, k}(x)}{d x^{2}}+a_{2^{j}+k}^{*} \frac{d^{2} \psi_{j, k}^{*}(x)}{d x^{2}}\right)+a_{N / 2} \frac{d^{2} \psi_{n-1}(x)}{d x^{2}}
$$

Onde

$$
\frac{d^{2} \psi_{j, k}(x)}{d x^{2}}=-4 \pi^{2} 2^{-j / 2} \sum_{m_{j}=2^{j}}^{2^{j+1}-1} m_{j}^{2} e^{2 \pi i m_{j}\left(x-\frac{k}{2^{j}}\right)}, \quad j=0,1, . ., n-2, \quad k=0,1, \ldots, 2^{j}-1
$$


e

$$
\frac{d^{2} \psi_{n-1}(x)}{d x^{2}}=-(N \pi)^{2} e^{-i N \pi x}
$$

Quanto às transformadas temos,

$$
\left(\frac{d^{2} \widehat{\psi_{j, k}(x)}}{d x^{2}}\right)_{w}= \begin{cases}(2 \pi i w)^{2} 2^{-j / 2} e^{-2 \pi i w \frac{w}{2^{j}}} & \text { quando } w=2^{j}, \ldots, 2^{j+1}-1 \\ 0 & \text { caso contrário }\end{cases}
$$

$\mathrm{e}$

$$
\left(\frac{d^{2} \widehat{\psi_{n-1}(x)}}{d x^{2}}\right)_{w}=(-N \pi i)^{2}, \quad w=-N / 2 .
$$

As mesmas relações de produtos internos nulos permanecem válidos, para ficarmos apenas com,

$$
\begin{gathered}
\left\langle\frac{d^{2} \psi_{n-1}(x)}{d x^{2}}, \psi_{n-1}(x)\right\rangle=-(N \pi)^{2} . \\
\chi_{k q}^{j}:=\left\langle\frac{d^{2} \psi_{j, k}(x)}{d x^{2}}, \psi_{j, q}(x)\right\rangle \\
=-4 \pi^{2} 2^{-j} \sum_{w=2^{j}}^{2^{j+1}-1} w^{2} e^{-2 \pi i w \frac{k-q}{2^{j}}} .
\end{gathered}
$$

Novamente temos propriedades a serem exploradas para melhorar a eficiência numérica, análogas as anteriores, porém valendo agora que

$$
\left(\chi_{q k}^{j}\right)^{*}=\chi_{k q}^{j}
$$

Ao projetarmos portanto $u_{x x}$ na base de ondaletas harmônicas vamos obter:

$$
\begin{aligned}
\left\langle u_{x x}, \phi\right\rangle & =0 \\
\left\langle u_{x x}, \psi_{r, s}(x)\right\rangle & =\sum_{k=0}^{2^{r}-1} a_{2^{r}+k} \chi_{k s}^{r}, \\
\left\langle u_{x x}, \psi_{n-1}(x)\right\rangle & =-(N \pi)^{2} a_{N / 2} .
\end{aligned}
$$

O algoritmo para se avaliar o conjunto completo da projeção de $u_{x x}$ na base de ondaletas harmônicas pode ser visto da mesma forma como no caso feito para $u_{x}$, gerando portanto um algoritmo com $O(N)$ de custo computacional, quando se força a restrição de suporte. 


\subsubsection{Projeção de não linearidades com derivadas}

Seja $v$ uma função em $L^{2}([0,1])$ escrita na base de ondaletas harmônicas,

$$
v(x)=a_{0}+\sum_{j=0}^{n-2} \sum_{k=0}^{2^{j}-1}\left(a_{2^{j}+k} \psi_{j, k}(x)+a_{2^{j}+k}^{*} \psi_{j, k}^{*}(x)\right)+a_{N / 2} \psi_{n-1}(x),
$$

e $u$ também uma função em $L^{2}([0,1])$ escrita na base de ondaletas harmônicas, mas para a qual vamos considerar sua derivada primeira,

$$
u_{x}=\sum_{p=0}^{n-2} \sum_{q=0}^{2^{p}-1}\left(b_{2^{p}+q} \frac{d \psi_{p, q}(x)}{d x}+b_{2^{p}+q}^{*} \frac{d \psi_{p, q}^{*}(x)}{d x}\right)+b_{N / 2} \frac{d \psi_{n-1}(x)}{d x}
$$

Queremos estudar a projeção da função $w=v u_{x}$ na base de ondaletas harmônicas,

$$
\begin{aligned}
w(x)= & \left(a_{0}+\sum_{j=0}^{n-2} \sum_{k=0}^{2^{j}-1}\left(a_{2^{j}+k} \psi_{j, k}(x)+a_{2^{j}+k}^{*} \psi_{j, k}^{*}(x)\right)+a_{N / 2} \psi_{n-1}(x)\right) \\
& \left(\sum_{p=0}^{n-2} \sum_{q=0}^{2^{p}-1}\left(b_{2^{p}+q} \frac{d \psi_{p, q}(x)}{d x}+b_{2^{p}+q}^{*} \frac{d \psi_{p, q}^{*}(x)}{d x}\right)+b_{N / 2} \frac{d \psi_{n-1}(x)}{d x}\right) .
\end{aligned}
$$

Note que desta forma incluímos o caso $w=u u_{x}$, bastando tomar $v=u$. Deixamos os cálculos como apêndice $(\mathrm{A}]$, e chegamos ao seguinte resultado para a projeção de $w$ :

$$
\begin{aligned}
\langle w, \phi\rangle & =\sum_{j=0}^{n-2} \sum_{k=0}^{2^{j}-1} \sum_{q=0}^{2^{j}-1}\left(a_{2^{j}+k} b_{2^{j}+q}^{*}\left(\gamma_{q k}^{j}\right)^{*}+a_{2^{j}+k}^{*} b_{2^{j}+q}\left(\gamma_{q k}^{j}\right)\right)-N \pi i a_{N / 2} b_{N / 2} \\
\left\langle w, \psi_{r, s}(x)\right\rangle & =a_{0} \sum_{q=0}^{2^{r}-1} b_{2^{r}+q} \gamma_{q s}^{r} \\
& +\sum_{j=0}^{n-2} \sum_{k=0}^{2^{j}-1} \sum_{p=0}^{n-2} \sum_{q=0}^{2^{p}-1}\left(a_{2^{j}+k} b_{2^{p}+q} P(0)_{k q s}^{j p r}+a_{2^{j}+k}^{*} b_{2^{p}+q} P(1)_{k q s}^{j p r}\right) \\
& +\sum_{j=0}^{n-2} \sum_{k=0}^{2^{j}-1} \sum_{p=0}^{n-2} \sum_{q=0}^{2^{p}-1}\left(a_{2^{j}+k} b_{2^{p}+q}^{*} P(2)_{k q s}^{j p r}\right) \\
\left\langle w, \psi_{n-1}(x)\right\rangle & =-N \pi i a_{0} b_{N / 2}+\sum_{j=0}^{n-2} \sum_{k=0}^{2^{j}-1} \sum_{p=0}^{n-2} \sum_{q=0}^{2^{p}-1}\left(a_{2^{j}+k}^{*} b_{2^{p}+q^{*}}^{*} N_{k q}^{j p *}\right)
\end{aligned}
$$

onde $\gamma_{q s}^{r}$ está definido como no caso linear de primeira ordem e 


$$
\begin{aligned}
& P(0)_{k q s}^{j p r}=\left\langle\psi_{j, k}(x) \frac{d \psi_{p, q}(x)}{d x}, \psi_{r, s}(x)\right\rangle= \\
& =\frac{2 \pi i}{2^{(j+p+r) / 2}} \sum_{m_{j}=2^{j}}^{2^{j+1}-1} \sum_{m_{p}=\max \left(2^{r}-m_{j}, 2^{p}\right)}^{\min \left(2^{r+1}-1-m_{j}, 2^{p+1}-1\right)} m_{p} e^{-2 \pi i\left(\frac{m_{j} k}{2^{j}}+\frac{m_{p} q}{2^{p}}-\frac{\left(m_{j}+m_{p}\right) s}{2^{r}}\right)}, \\
& P(1)_{k q s}^{j p r}=\left\langle\psi_{j, k}^{*}(x) \frac{d \psi_{p, q}(x)}{d x}, \psi_{r, s}(x)\right\rangle= \\
& =\frac{2 \pi i}{2^{(j+p+r) / 2}} \sum_{m_{j}=2^{j}}^{2^{j+1}-1} \sum_{m_{p}=\max \left(2^{r}+m_{j}, 2^{p}\right)}^{\min \left(2^{r+1}-1+m_{j}, 2^{p+1}-1\right)} m_{p} e^{-2 \pi i\left(-\frac{m_{j} k}{2^{j}}+\frac{m_{p} q}{2^{p}}-\frac{\left(m_{p}-m_{j}\right) s}{2^{r}}\right)}, \\
& P(2)_{k q s}^{j p r}=\left\langle\psi_{j, k}(x) \frac{d \psi_{p, q}^{*}(x)}{d x}, \psi_{r, s}(x)\right\rangle= \\
& =-\frac{2 \pi i}{2^{(j+p+r) / 2}} \sum_{m_{j}=2^{j}}^{2^{j+1}-1} \sum_{m_{p}=\max \left(m_{j}-\left(2^{r+1}-1\right), 2^{p}\right)}^{\min \left(m_{j}-2^{r}, 2^{p+1}-1\right)} m_{p} e^{-2 \pi i\left(\frac{m_{j} k}{2^{j}}-\frac{m_{p} q}{2^{p}}-\frac{\left(m_{j}-m_{p}\right) s}{2^{r}}\right)}, \\
& N_{k q}^{j p}=\left\langle\psi_{j, k}^{*}(x) \frac{d \psi_{p, q}^{*}(x)}{d x}, \psi_{n-1}(x)\right\rangle=\frac{2 \pi i}{2^{(j+p) / 2}} \sum_{m_{p}=\max \left(N / 2-\left(2^{j+1}-1\right), 2^{p}\right)}^{\min \left(N / 2-2^{j}, 2^{p+1}-1\right)} m_{p} e^{2 \pi i m_{p}\left(\frac{k}{2^{j}}-\frac{q}{2^{p}}\right)},
\end{aligned}
$$

são os coeficientes de conexão não lineares de ordem 1 e podem ser previamente calculados para o método de Galerkin.

Vamos agora olhar para o custo computacional de se projetar $w$ na base de ondaletas harmônicas. Faremos isso olhando para cada parcela de cálculos de (3.64) até (3.68).

1. Operações de 3.64 : $\frac{N}{2}$ devido ao somatório em $j$ e $k$, acrescido de no máximo $\frac{N}{4}$ para o somatório de $q$, resultando em $O\left(N^{2}\right)$ operações.

2. Operações de (3.65): Análogo ao caso da projeção de $u_{x}$, resultando em $O(N)$ operações.

3. Operações de 3.66 e 3.67$):\left(\frac{N}{2}\right)^{2}$ devido ao somatório em $j, k$ e $p, q$, resultando em $O\left(N^{2}\right)$ operações.

4. Operações de 3.68): $\left(\frac{N}{2}\right)^{2}$ devido ao somatório em $j, k$ e $p, q$, resultando em $O\left(N^{2}\right)$ operações.

Agora lembrando que temos que realizar cálculos para $r=0,1, \ldots, n-2$ e $s=0,1, \ldots, 2^{r}-1$, teremos um custo total da ordem de $N^{3}$ operações. O custo computacional está basicamente determinado pelos coeficientes de conexão não lineares, porém estes possuem uma estrutura de esparsidade que deve ser aproveitada nos cálculos da projeção. Vamos analisar os valores de $\left|P(0)_{k q s}^{j p r}\right|$ 
para alguns pares $(r, s)$. No caso $r=0$ e $s=0, P(0)$ é nulo para quaisquer valores de $j, k, p$ e $q$. Podemos ver os casos tais que $r>0$ na figura 3.1, onde nota-se claramente uma predominância de valores nulos, ou próximos de zero. Mostramos na figura 3.2 os valores $\operatorname{de} \log _{2}\left(\left|P(0)_{k q s}^{j p r}\right|+1\right)$, para vermos mais detalhes da esparsidade quando $P(0)$ tem valores pequenos.
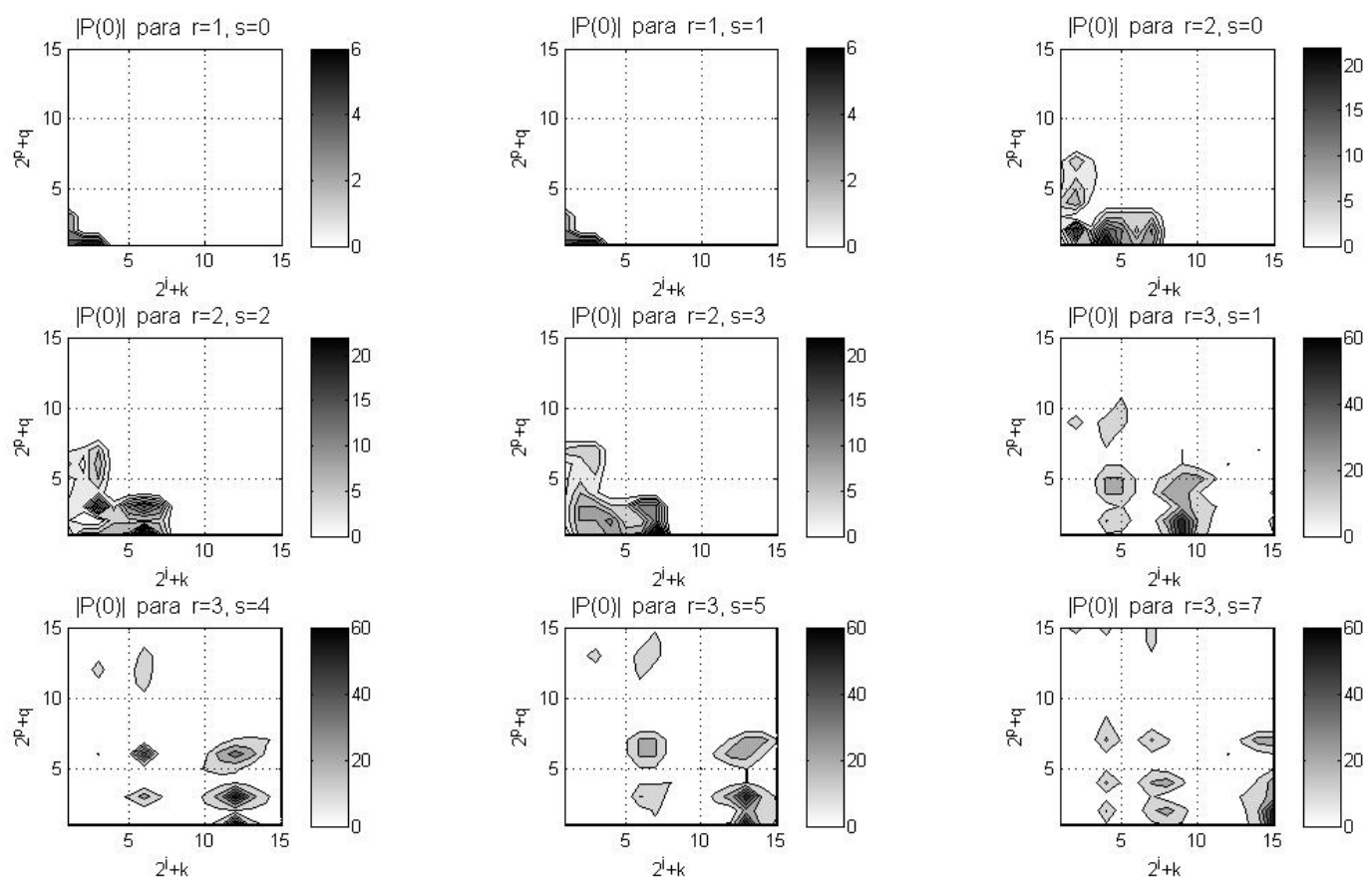

Figura 3.1: Valores absolutos dos coeficientes de conexão não lineares $P(0)_{k q s}^{j p r}$ para alguns pares $(r, s)$. No eixo $x$ estão os índices $(j, k)$ seguindo a ordem dada por $2^{j}+k$, e no eixo $y$ os índices $(p, q)$ seguindo a ordem $2^{p}+q$

Os coeficientes de conexão não lineares $P(1)$ e $P(2)$ seguem padrões de esparsidade parecidos com os de $P(0)$, com predominância de valores nulos, ou próximos de zero. Para o caso da projeção na direção de $\psi_{n-1}(x)$ o custo computacional é predominantemente dado pelo coeficiente de conexão não linear $N_{k q}^{j p}$, que também tem maioria de valores nulos, como pode ser visto na figura 3.3

Porém, assim como no caso da projeção de $u_{x}$, podemos usar as propriedades de localidade para reduzir o custo computacional. Tomemos um coeficiente de corte $d=1$, considerado na expressão 2.25, para o suporte de ondaletas harmônicas, e para suas derivadas, e vamos supor 

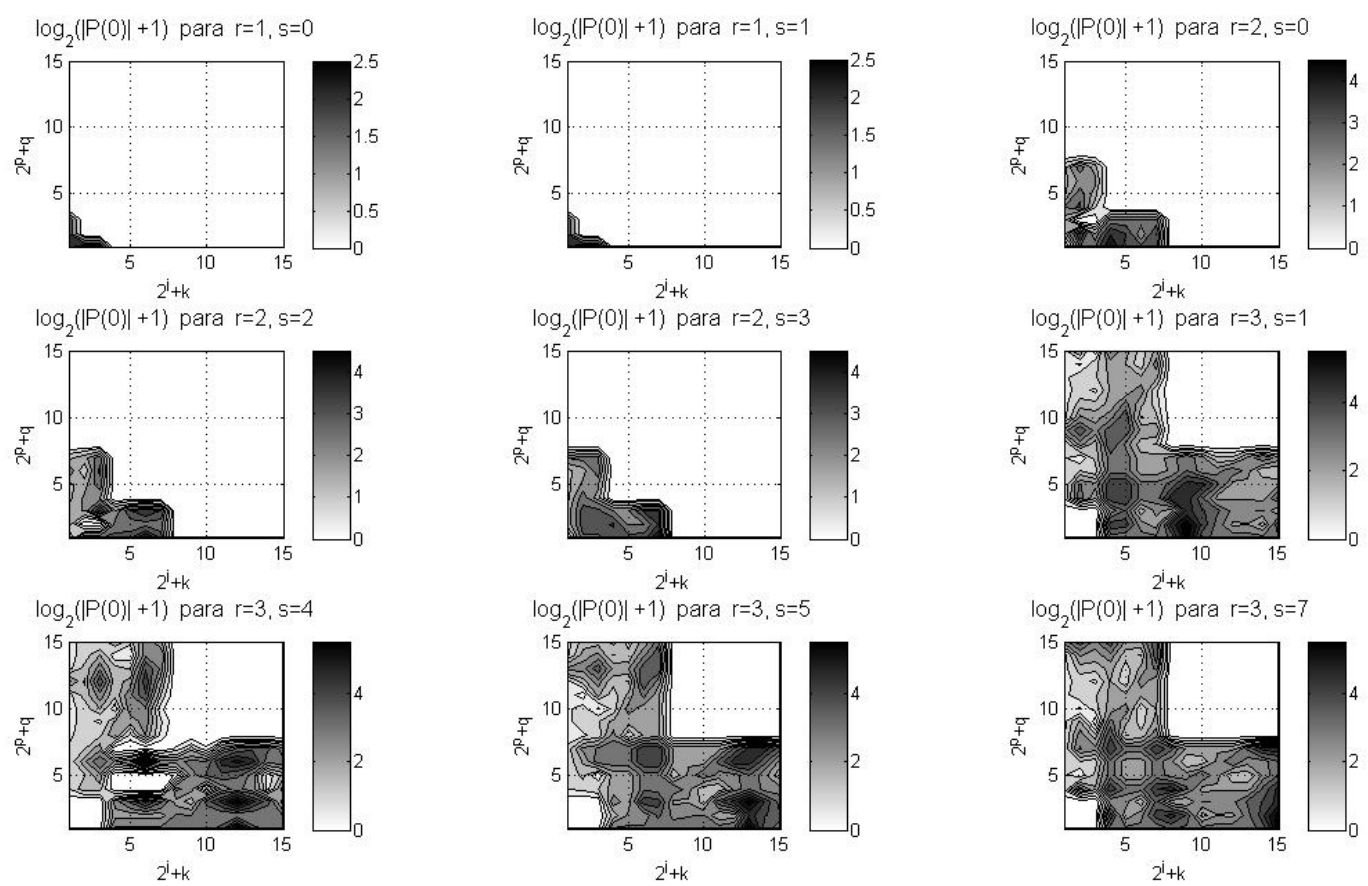

Figura 3.2: Valores absolutos dos coeficientes de conexão não lineares $P(0)_{k q s}^{j p r}$ em logaritmo para alguns pares $(r, s)$. No eixo $x$ estão os índices $(j, k)$ seguindo a ordem dada por $2^{j}+k$, e no eixo $y$ os índices $(p, q)$ seguindo a ordem $2^{p}+q$

que tanto a função de ondaleta harmônica, quando sua derivada devem ter suporte com intersecção no suporte do elemento da base o qual estamos projetando. Novamente note que só estamos usando a restrição de suporte para definir quais coeficientes de conexão não lineares serão desprezados, mas no cálculo daqueles que serão considerados é utilizado o suporte das ondaletas harmônicas sobre todo o intervalo. Mostramos na figura 3.4 o padrão de esparsidade de $P(0)$, em escala logarítmica, com o coeficiente de corte $d=1$, onde nota-se um aumento da região com valores nulos.

No caso de termos $v=u$ temos ainda uma simplificação caso $u$ seja periódica em $[0,1]$

$$
\begin{aligned}
\left\langle u u_{x}, 1\right\rangle & =\int_{0}^{1} u u_{x} d x=\left[u^{2}(x)\right]_{x=0}^{1}-\int_{0}^{1} u_{x} u d x \\
& \Leftrightarrow \\
\left\langle u u_{x}, 1\right\rangle & =0 .
\end{aligned}
$$

Assim como no caso de Fourier, é possível obtermos um método rápido (com custo computacional da ordem de $O(N \log N)$ ) para calcularmos esta projeção, com um método pseudo-espectral. Dados os coeficientes de ondaletas de $v$ e $u_{x}$, transformamos estes coeficientes em valores na malha 

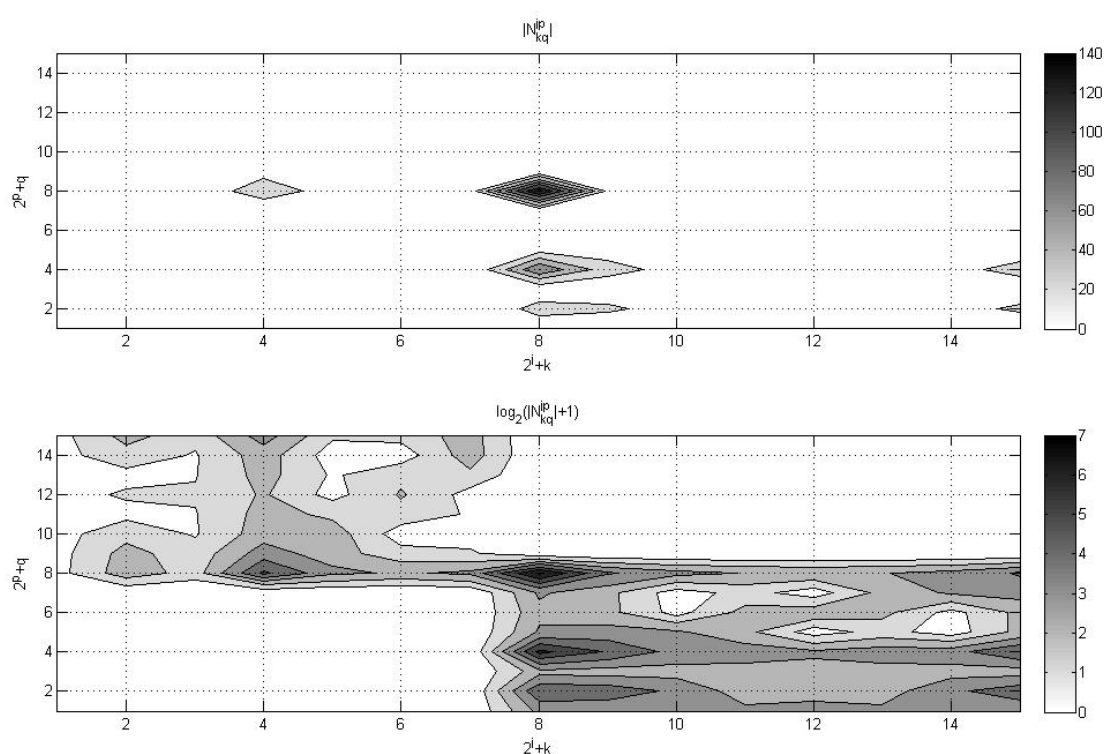

Figura 3.3: Valores absolutos dos coeficientes de conexão não lineares $N_{k q}^{j p}$ e $\operatorname{de} \log _{2}\left(\left|N_{k q}^{j p}\right|+1\right)$. No eixo $x$ estão os índices $(j, k)$ seguindo a ordem dada por $2^{j}+k$, e no eixo $y$ os índices $(p, q)$ seguindo a ordem $2^{p}+q$

numérica, aonde efetuamos o produto, e depois voltamos para o domínio de coeficientes de ondaletas harmônicas. Porém aqui encontramos alguns problemas. Primeiro que temos os coeficientes de ondaletas de $u$, e não de $u_{x}$, como seria desejável. Para resolver este problema desenvolvemos um algoritmo rápido para a transformada direta e inversa da derivada de uma função. Mais especificamente, se são dados os valores da derivada de uma função na malha, calculamos os coeficientes de ondaletas da função. Por outro lado, se são dados os coeficientes de ondaletas de uma função, calculamos os valores da derivada da função na malha. As deduções deste método seguem no apêndice B. Isto posto, ainda temos que resolver o problema de "aliasing". Resolvemos este problema de forma análoga ao caso de Fourier. Consideramos $M=3 N / 2$ pontos de malha, e ao realizarmos as transformadas de ondaletas harmônicas, no passo intermediário (veja figura 2.8) zeramos os coeficientes de Fourier nas frequências mais altas. O segundo passo da transformada é feito apenas com os elementos de frequências mais baixas (menores que $\frac{N}{2}$ ). No caso da transformada inversa, adicionamos zeros nas frequências mais altas, para obter uma transformada inversa consistente com a direta. Desta forma eliminamos o problema de "aliasing" assim como foi feito no caso de Fourier.

\subsubsection{Problema teste}

Vamos considerar um problema teste, idêntico ao anterior, que utilize as projeções definidas anteriormente, mas agora para ilustrar o método de Galerkin-Ondaleta utilizando a base de ondaletas harmônicas. 

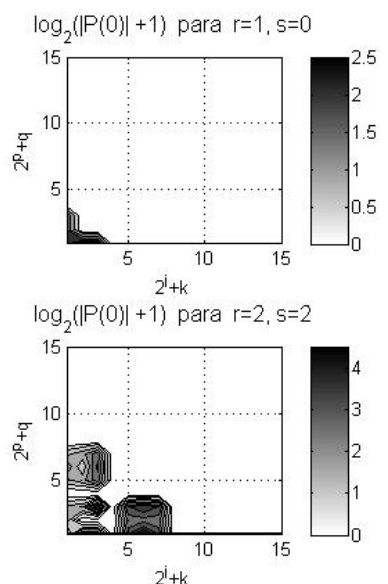

$\log _{2}(|P(0)|+1)$ para $r=3, s=4$

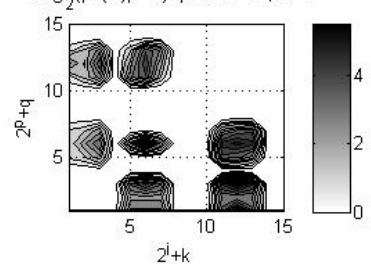

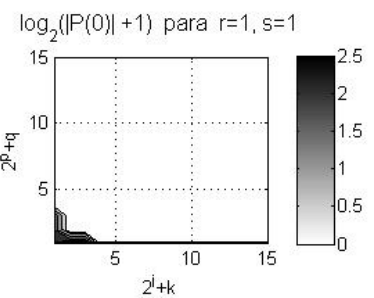

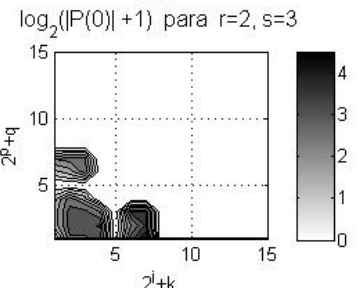

$\log _{2}\{|P(0)|+1)$ para $r=3, s=5$

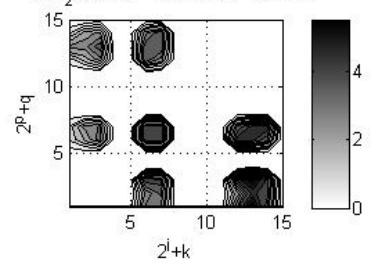

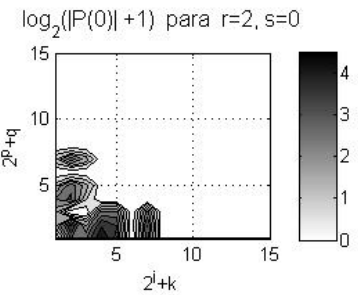

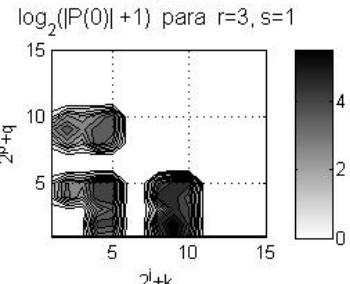

$\log _{2}(|P(0)|+1)$ para $r=3, s=7$

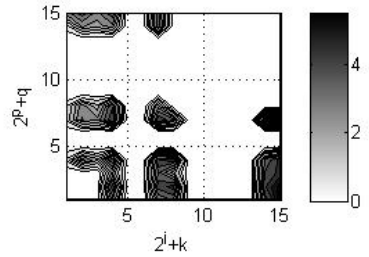

Figura 3.4: Logaritmo dos valores absolutos dos coeficiente de conexão não lineares $P(0), \log _{2}\left(\left|P(0)_{k q s}^{j p r}\right|+1\right)$, usando localidade do suporte da base, com parâmetro $d=1$, para alguns pares $(r, s)$. No eixo $x$ estão os índices $(j, k)$ seguindo a ordem dada por $2^{j}+k$, e no eixo $y$ os índices $(p, q)$ seguindo a ordem $2^{p}+q$

Considere o problema de valor inicial,

$$
\begin{cases}D(u(x, t))=\frac{\partial u(x, t)}{\partial t}+c \frac{\partial u(x, t)}{\partial x}+v(x, t) \frac{\partial u(x, t)}{\partial x}-\nu \frac{\partial^{2} u(x, t)}{\partial x^{2}}=0, & x \in(0,1), \\ u(x, 0)=f(x), & x \in[0,1], \\ u(0, t)=u(1, t), & t \geq 0,\end{cases}
$$

onde $f(x) \in L^{2}([0,1])$ e,$v(x, t) \in L^{2}([0,1])$ na variável $x$, para cada $t$ fixado. Consideramos ainda a possibilidade de tomarmos $v=u$. Buscamos uma aproximação para a solução exata $u(x, t)$, para tanto vamos definir $u_{N}(x, t)$ utilizando separação de variáveis e expansão em série de ondaletas harmônicas, dada por

$$
u_{N}(x, t)=a_{0}(t)+\sum_{j=0}^{n-2} \sum_{k=0}^{2^{j}-1}\left(a_{2^{j}+k}(t) \psi_{j, k}(x)+a_{2^{j}+k}^{*}(t) \psi_{j, k}^{*}(x)\right)+a_{N / 2}(t) \psi_{n-1}(x),
$$

e as expansões em ondaletas harmônicas de $v$ e $f$, 


$$
\begin{gathered}
v_{N}(x, t)=b_{0}(t)+\sum_{j=0}^{n-2} \sum_{k=0}^{2^{j}-1}\left(b_{2^{j}+k}(t) \psi_{j, k}(x)+b_{2^{j}+k}(t)^{*} \psi_{j, k}^{*}(x)\right)+b_{N / 2}(t) \psi_{n-1}(x), \\
f_{N}(x)=d_{0}+\sum_{j=0}^{n-2} \sum_{k=0}^{2^{j}-1}\left(d_{2^{j}+k} \psi_{j, k}(x)+d_{2^{j}+k}^{*} \psi_{j, k}^{*}(x)\right)+d_{N / 2} \psi_{n-1}(x) .
\end{gathered}
$$

Pelo método de Galerkin-Ondaleta temos que resolver novamente um sistema não linear da forma,

$$
\begin{aligned}
\left\langle D u_{N}, 1\right\rangle_{L^{2}([0,1])} & =0 \\
\left\langle D u_{N}, \psi_{r, s}(x)\right\rangle_{L^{2}([0,1])} & =0, \quad r=0,1, \ldots, n-2, s=0,1, . ., 2^{r}-1, \\
\left\langle D u_{N}, \psi_{n-1}(x)\right\rangle_{L^{2}([0,1])} & =0
\end{aligned}
$$

onde $D u_{N}$ será dado pela expressão,

$$
D u_{N}(x, t)=\frac{\partial u_{N}(x, t)}{\partial t}+c \frac{\partial u_{N}(x, t)}{\partial x}+v_{N}(x, t) \frac{\partial u_{N}(x, t)}{\partial x}-\nu \frac{\partial^{2} u_{N}(x, t)}{\partial x^{2}} .
$$

Vamos usar o que foi desenvolvido nas últimas seções para construir o sistema não linear. Omitiremos um pouco de notação para facilitar a visualização das equações. Começando pelo elemento da base $\phi(x)=1$ temos,

$$
\left\langle D u_{N}, 1\right\rangle=\left\langle\frac{\partial u_{N}(x, t)}{\partial t}, 1\right\rangle+c\left\langle\frac{\partial u_{N}(x, t)}{\partial x}, 1\right\rangle+\left\langle v_{N}(x, t) \frac{\partial u_{N}(x, t)}{\partial x}, 1\right\rangle-\nu\left\langle\frac{\partial^{2} u_{N}(x, t)}{\partial x^{2}}, 1\right\rangle,
$$

onde a primeira parcela será dada por

$$
\left\langle\frac{\partial u_{N}(x, t)}{\partial t}, 1\right\rangle=\frac{d a_{0}(t)}{d t}
$$

e as demais foram construídas nas seções anterior, resultando em

$$
\begin{gathered}
\left\langle\frac{\partial u_{N}(x, t)}{\partial x}, 1\right\rangle=0 \\
\left\langle v_{N}(x, t) \frac{\partial u_{N}(x, t)}{\partial x}, 1\right\rangle=\sum_{j=0}^{n-2} \sum_{k=0}^{2^{j}-1} \sum_{q=0}^{2^{j}-1}\left(b_{2^{j}+k}(t) a_{2^{j}+q}^{*}(t)\left(\gamma_{q k}^{j}\right)^{*}+b_{2^{j}+k}^{*}(t) a_{2^{j}+q}(t)\left(\gamma_{q k}^{j}\right)\right) \\
-N \pi i b_{N / 2}(t) a_{N / 2}(t),
\end{gathered}
$$




$$
\left\langle\frac{\partial^{2} u_{N}(x, t)}{\partial x^{2}}, 1\right\rangle=0
$$

e por fim teremos que,

$$
\begin{aligned}
\frac{d a_{0}(t)}{d t} & =-\sum_{j=0}^{n-2} \sum_{k=0}^{2^{j}-1} \sum_{q=0}^{2^{j}-1}\left(b_{2^{j}+k}(t) a_{2^{j}+q}^{*}(t)\left(\gamma_{q k}^{j}\right)^{*}+b_{2^{j}+k}^{*}(t) a_{2^{j}+q}(t)\left(\gamma_{q k}^{j}\right)\right) \\
& +N \pi i b_{N / 2}(t) a_{N / 2}(t) .
\end{aligned}
$$

Agora para o elemento $\psi_{r, s}(x)$ da base,

$$
\begin{aligned}
\left\langle D u_{N}, \psi_{r, s}(x)\right\rangle & =\left\langle\frac{\partial u_{N}(x, t)}{\partial t}, \psi_{r, s}(x)\right\rangle+c\left\langle\frac{\partial u_{N}(x, t)}{\partial x}, \psi_{r, s}(x)\right\rangle \\
& +\left\langle v_{N}(x, t) \frac{\partial u_{N}(x, t)}{\partial x}, \psi_{r, s}(x)\right\rangle-\nu\left\langle\frac{\partial^{2} u_{N}(x, t)}{\partial x^{2}}, \psi_{r, s}(x)\right\rangle
\end{aligned}
$$

onde teremos,

$$
\begin{aligned}
&\left\langle\frac{\partial u_{N}(x, t)}{\partial t}, \psi_{r, s}(x)\right\rangle=\frac{d a_{2^{r}+s}(t)}{d t} \\
&\left\langle\frac{\partial u_{N}(x, t)}{\partial x}, \psi_{r, s}(x)\right\rangle=\sum_{k=0}^{2^{r}-1} a_{2^{r}+k}(t) \gamma_{k s}^{r}
\end{aligned}
$$

$$
\left\langle\frac{\partial^{2} u_{N}(x, t)}{\partial x^{2}}, \psi_{r, s}(x)\right\rangle=\sum_{k=0}^{2^{r}-1} a_{2^{r}+k}(t) \chi_{k s}^{r},
$$


resultando em,

$$
\begin{aligned}
\frac{d a_{2^{r}+s}(t)}{d t} & =-c \sum_{k=0}^{2^{r}-1} a_{2^{r}+k}(t) \gamma_{k s}^{r}-b_{0}(t) \sum_{q=0}^{2^{r}-1} a_{2^{r}+q}(t) \gamma_{q s}^{r} \\
& -\sum_{j=0}^{n-2} \sum_{k=0}^{2^{j}-1} \sum_{p=0}^{n-2} \sum_{q=0}^{2^{p}-1}\left(b_{2^{j}+k}(t) a_{2^{p}+q}(t) P(0)_{k q s}^{j p r}+b_{2^{j}+k}^{*}(t) a_{2^{p}+q}(t) P(1)_{k q s}^{j p r}\right) \\
& -\sum_{j=0}^{n-2} \sum_{k=0}^{2^{j}-1} \sum_{p=0}^{n-2} \sum_{q=0}^{2^{p}-1}\left(b_{2^{j}+k}(t) a_{2^{p}+q}^{*}(t) P(2)_{k q s}^{j p r}\right)+\nu \sum_{k=0}^{2^{r}-1} a_{2^{r}+k}(t) \chi_{k s}^{r}
\end{aligned}
$$

Para o elemento restante da base, $\psi_{n-1}(x)$,

$$
\begin{aligned}
\left\langle D u_{N}, \psi_{n-1}(x)\right\rangle & =\left\langle\frac{\partial u_{N}(x, t)}{\partial t}, \psi_{n-1}(x)\right\rangle+c\left\langle\frac{\partial u_{N}(x, t)}{\partial x}, \psi_{n-1}(x)\right\rangle \\
& +\left\langle v_{N}(x, t) \frac{\partial u_{N}(x, t)}{\partial x}, \psi_{n-1}(x)\right\rangle-\nu\left\langle\frac{\partial^{2} u_{N}(x, t)}{\partial x^{2}}, \psi_{n-1}(x)\right\rangle=0
\end{aligned}
$$

onde teremos,

$$
\begin{aligned}
\left\langle\frac{\partial u_{N}(x, t)}{\partial t}, \psi_{n-1}(x)\right\rangle & =\frac{d a_{N / 2}(t)}{d t}, \\
\left\langle\frac{\partial u_{N}(x, t)}{\partial x}, \psi_{n-1}(x)\right\rangle & =-N \pi i a_{N / 2}(t), \\
\left\langle v_{N}(x, t) \frac{\partial u_{N}(x, t)}{\partial x}, \psi_{n-1}(x)\right\rangle & =-N \pi i b_{0}(t) a_{N / 2}(t) \\
& +\sum_{j=0}^{n-2} \sum_{k=0}^{2^{j}-1} \sum_{p=0}^{n-2} \sum_{q=0}^{2^{p}-1}\left(b_{2^{j}+k}^{*}(t) a_{2^{p}+q}^{*}(t) N_{k q}^{j p *}\right) \\
\left\langle\frac{\partial^{2} u_{N}(x, t)}{\partial x^{2}}, \psi_{n-1}(x)\right\rangle & =-(N \pi)^{2} a_{N / 2}(t),
\end{aligned}
$$

resultando em,

$$
\begin{aligned}
\frac{d a_{N / 2}(t)}{d t} & =c N \pi i a_{N / 2}(t)+N \pi i b_{0}(t) a_{N / 2}(t) \\
& -\sum_{j=0}^{n-2} \sum_{k=0}^{2^{j}-1} \sum_{p=0}^{n-2} \sum_{q=0}^{2^{p}-1}\left(b_{2^{j}+k}^{*}(t) a_{2^{p}+q}^{*}(t) N_{k q}^{j p *}\right) \\
& -\nu(N \pi)^{2} a_{N / 2} .
\end{aligned}
$$


Com isso podemos montar o sistema não linear a ser resolvido,

$$
\left\{\begin{aligned}
\frac{d a_{0}(t)}{d t} & =-\sum_{j=0}^{n-2} \sum_{k=0}^{2^{j}-1} \sum_{q=0}^{2^{j}-1}\left(b_{2^{j}+k}(t) a_{2^{j}+q}^{*}(t)\left(\gamma_{q k}^{j}\right)^{*}+b_{2^{j}+k}^{*}(t) a_{2^{j}+q}(t)\left(\gamma_{q k}^{j}\right)\right) \\
& +N \pi i b_{N / 2}(t) a_{N / 2}(t), \\
\frac{d a_{2^{r}+s}(t)}{d t} & =-c \sum_{k=0}^{2^{r}-1} a_{2^{r}+k}(t) \gamma_{k s}^{r}-b_{0}(t) \sum_{q=0}^{2^{r}-1} a_{2^{r}+q}(t) \gamma_{q s}^{r} \\
& -\sum_{j=0}^{n-2} \sum_{k=0}^{2^{j}-1} \sum_{p=0}^{n-2} \sum_{q=0}^{2^{p}-1}\left(b_{2^{j}+k}(t) a_{2^{p}+q}(t) P(0)_{k q s}^{j p r}+b_{2^{j}+k}^{*}(t) a_{2^{p}+q}(t) P(1)_{k q s}^{j p r}\right) \\
& -\sum_{j=0}^{n-2} \sum_{k=0}^{2^{j}-1} \sum_{p=0}^{n-2} \sum_{q=0}^{2^{p}-1}\left(b_{2^{j}+k}(t) a_{2^{p}+q}^{*}(t) P(2)_{k q s}^{j p r}\right) \\
& +\nu \sum_{k=0}^{2^{r}-1} a_{2^{r}+k}(t) \chi_{k s}^{r} \\
& \operatorname{para} r=0,1, \ldots, n-2, \quad s=0,1, \ldots, 2^{r}-1, \\
\frac{d a_{N / 2}(t)}{d t} & =c N \pi i a_{N / 2}(t)+N \pi i b_{0}(t) a_{N / 2}(t) \\
& -\sum_{j=0}^{n-2} \sum_{k=0}^{2^{j}-1} \sum_{p=0}^{n-2} \sum_{q=0}^{2^{p}-1}\left(b_{2^{j}+k}^{*}(t) a_{2^{p}+q}^{*}(t) N_{k q}^{j p *}\right) \\
& -\nu(N \pi)^{2} a_{N / 2}(t) .
\end{aligned}\right.
$$




\section{Capítulo 4}

\section{Testes Numéricos}

Neste capítulo vamos realizar uma série de testes numéricos para o método de Galerkin com ondaletas harmônicas. A plataforma de programação usada nos experimentos numéricos foi o Matlab ${ }^{\circledR}$ versão 7.6.0(R2008a). A maior parte dos experimentos foram executados em um computador com processador Intel ${ }^{\circledR}$ Core $^{T M} 2$ Duo com $2 \mathrm{GHz}$ de frequência e $2 \mathrm{~GB}$ de memória RAM.

Vamos considerar alguns casos particulares do problema de valor inicial

$$
\begin{cases}D(u(x, t))=\frac{\partial u(x, t)}{\partial t}+c \frac{\partial u(x, t)}{\partial x}+v(x, t) \frac{\partial u(x, t)}{\partial x}-\nu \frac{\partial^{2} u(x, t)}{\partial x^{2}}=0, & x \in(0,1) \\ u(x, 0)=f(x), & x \in[0,1] \\ u(0, t)=u(1, t), & t \geq 0,\end{cases}
$$

onde $f(x) \in L^{2}([0,1])$ e , $v(x, t) \in L^{2}([0,1])$ na variável $x$, para cada $t$ fixado. Tomaremos $f(x)$ com sendo uma das funções abaixo

1. Seno

$$
f(x)=\sin (2 \pi x)
$$

2. Exponencial

$$
f(x)=e^{-\left((x-0.3)^{4}\right) 10^{-5}}+1 / 2
$$

3. Pulso

$$
f(x)=(\sin (\pi(x+0.4)))^{80}
$$

Queremos resolver alguns casos particulares deste problema utilizando o método de Galerkin com bases de ondaletas harmônicas e de Fourier. Vamos analisar o comportamento do problema apenas com advecção linear e posteriormente com advecção não linear e difusão. 


\subsection{Advecção Linear}

Iniciamos com $c=1, v(x, t)=0, \nu=0$. Com estes parâmetros reduzimos o problema a uma equação do transporte, com condição inicial dada por $f(x)$, e velocidade de transporte igual a um,

$$
\begin{cases}D(u(x, t))=\frac{\partial u(x, t)}{\partial t}+\frac{\partial u(x, t)}{\partial x}=0, & x \in(0,1) \\ u(x, 0)=f(x), & x \in[0,1] \\ u(0, t)=u(1, t), & t \geq 0,\end{cases}
$$

Neste caso conhecemos a solução exata, que é dada por $u(x, t)=f(x-t)$, portanto podemos fazer comparações diretamente com a solução exata do problema. A resolução do problema pelo método de Galerkin com base de Fourier consiste na resolução do sistema de equações diferenciais

$$
\begin{cases}\frac{\partial a_{\lambda}(t)}{\partial t}=-2 \pi i \lambda a_{\lambda}(t) & \\ a_{\lambda}(0)=b_{\lambda}, & \lambda=-N / 2, \ldots, N / 2-1,\end{cases}
$$

onde consideramos a aproximação $u_{N}$ da solução e a condição inicial $f$ escritas na base de Fourier,

$$
u_{N}(x, t)=\sum_{w=-N / 2}^{N / 2-1} a_{w}(t) e^{2 \pi i w x}
$$

$\mathrm{e}$

$$
f_{N}(x)=\sum_{k=-N / 2}^{N / 2-1} b_{k} e^{2 \pi i k x}
$$

para o qual também conhecemos a solução exata, dada por $a_{\lambda}(t)=b_{\lambda} e^{-2 \pi i \lambda t}$ para $\lambda=-N / 2, \ldots, N / 2-$ 1.

Para a base de ondaletas harmônicas, considerando as aproximações $u_{N}$ e $f_{N}$,

$$
\begin{gathered}
u_{N}(x, t)=a_{0}(t)+\sum_{j=0}^{n-2} \sum_{k=0}^{2^{j}-1}\left(a_{2^{j}+k}(t) \psi_{j, k}(x)+a_{2^{j}+k}^{*}(t) \psi_{j, k}^{*}(x)\right)+a_{N / 2}(t) \psi_{n-1}(x), \\
f_{N}(x)=d_{0}+\sum_{j=0}^{n-2} \sum_{k=0}^{2^{j}-1}\left(d_{2^{j}+k} \psi_{j, k}(x)+d_{2^{j}+k}^{*} \psi_{j, k}^{*}(x)\right)+d_{N / 2} \psi_{n-1}(x) .
\end{gathered}
$$


vamos precisar resolver o sistema de equações diferenciais,

$$
\begin{cases}\frac{d a_{0}(t)}{d t} & =0, \\ \frac{d a_{2^{r}+s}(t)}{d t} & =-\sum_{k=0}^{2^{r}-1} a_{2^{r}+k}(t) \gamma_{k s}^{r}, \quad \text { para } r=0,1, \ldots, n-2, \quad s=0,1, \ldots, 2^{r}-1, \\ \frac{d a_{N / 2}(t)}{d t} & =N \pi i a_{N / 2}(t),\end{cases}
$$

com condições iniciais dadas por $a_{k}(0)=d_{k}$, para $k=0,1, \ldots, N / 2$.

Começamos com um teste numérico tomando $n=7$, isto é, $N=2^{7}=128$ pontos de malha no espaço. Usamos um método de Runge-Kutta de quarta ordem para a discretização temporal dos sistemas de EDOs. Adotamos $\Delta x=1 / N$ e $\Delta t=\Delta x / 8$, para garantir estabilidade do método Runge-Kutta (consulte Canuto et al. (1988) para detalhes). Consideramos como condição inicial a função denominada anteriormente por exponencial (equação (4.3)). Na figura 4.1 mostramos 4 instantes de tempo da solução numérica por ondaletas harmônicas em relação à solução exata e ao método baseado em Fourier, com $\mathrm{N}=128$. Neste primeiro experimento não foi considerada a característica de localidade do suporte de ondaletas harmônicas, com isso é possível notarmos a equivalência entre os métodos fundamentados em ondaletas harmônicas e Fourier. Em seguida mostramos na figura 4.2 a evolução do erro, em relação à solução exata, e dos coeficientes de Fourier e de ondaletas harmônicas. Nota-se claramente a característica de localidade dos coeficientes de ondaletas harmônicas que tendem a acompanhar a evolução do sinal, em contrapartida com os coeficientes de Fourier, nos quais não se observa localidade do sinal. 

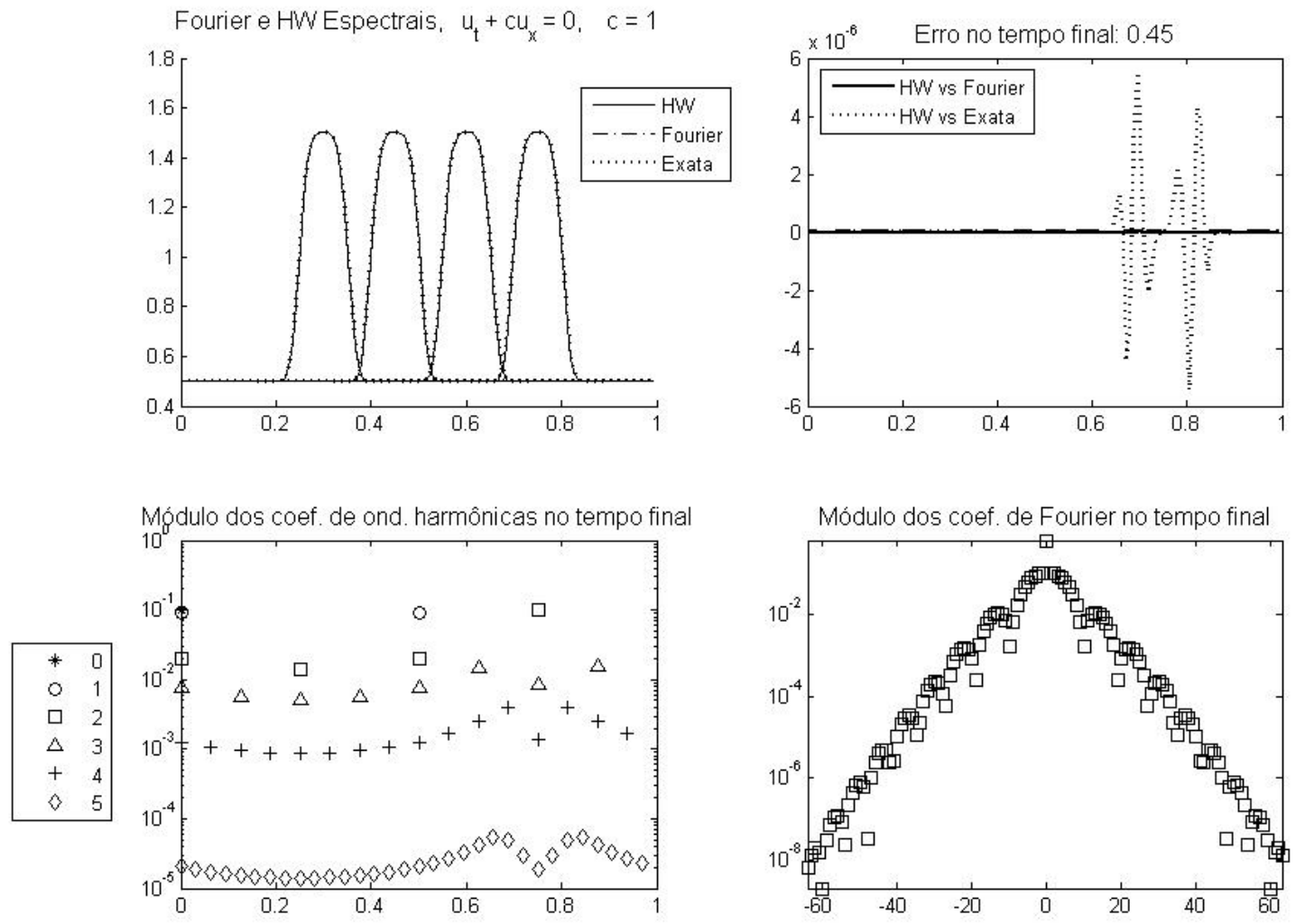

Parâmetros: $d t / d x=0.125 \mathrm{dt}=0.00097656 \quad \mathrm{~N}=128 \quad$ Niveis $\log 2(\mathrm{~N})=7 \quad$ Gráfico a cada 0.15 Cortes: Linear $(\mathrm{d})=0$ Não Linear $(\mathrm{dnl})=0$ Freq. $=0$ Geral $(\mathrm{rc})=0$

Figura 4.1: Advecção de função exponencial com ondaletas harmônicas considerando N=128 pontos de malha. No canto superior esquerdo aparecem as soluções exata e aproximadas pelo método de Fourier e de ondaletas harmônicas (HW) em 4 instantes de tempo $(0,0.15,0.3,0.45)$, e no lado direito aparecem as diferenças entre a solução por ondaletas harmônicas (HW) e a solução exata, e em relação à Fourier no tempo final (.45). Na parte inferior estão os módulos dos coeficientes de ondaletas harmônicas por níveis (esquerda) e Fourier (direita) no tempo final (.45)

Ao considerarmos a caracteristica de localidade do suporte das ondaletas harmônicas as diferenças com o método fundamentado em Fourier começam a aparecer. Na figura 4.3 , onde consideramos um coeficiente de corte para o suporte de 1 (veja equação 2.25) nota-se o aparecimento de oscilações de baixa amplitude em locais onde a solução deveria ser nula. Isso reflete exatamente a parte das ondaletas harmônicas eliminadas ao utilizarmos apenas um subintervalo de suporte para as mesmas, que sabemos ser aproximadamente $10 \%$ de seu suporte em norma. Ao tomarmos, por exemplo, $d=3$, temos uma melhora da aproximação da solução numérica em relação à exata, que mostramos na figura 4.4 . 

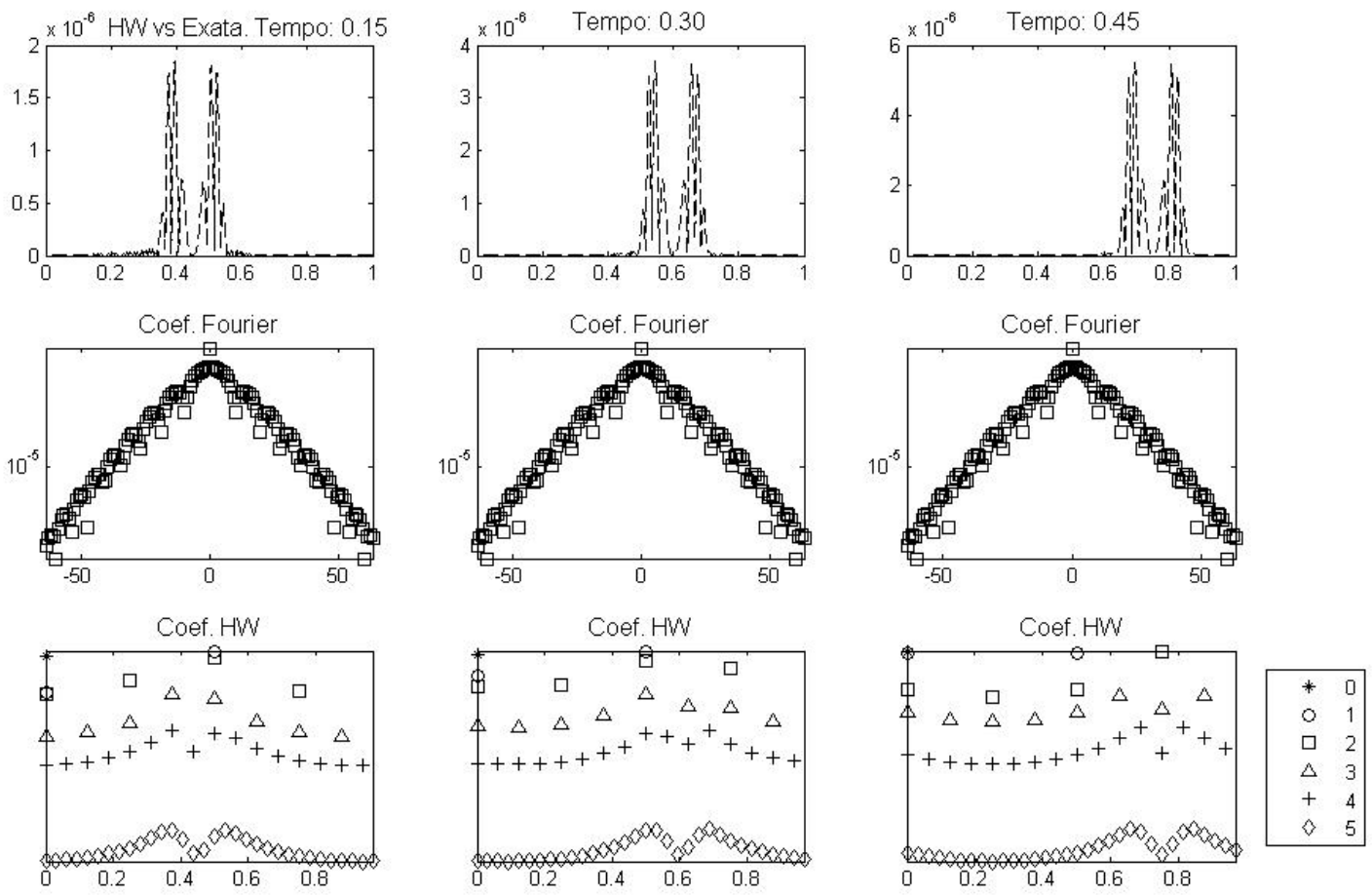

Advecçăo Linear: $d t / d x=0.125 \quad \mathrm{~N}=128$ Niveis $\log 2(\mathrm{~N})=7 \quad$ Cortes: Linear $(\mathrm{d})=0$ Năo Linear $(\mathrm{dnl})=0$ Freq. $=0$ Geral $(\mathrm{rc})=0$

Figura 4.2: Advecção de função exponencial com ondaletas harmônicas considerando $\mathrm{N}=128$ pontos de malha. Na primeira linha aparecem os erros entre as aproximações obtidas com ondaletas harmônicas e a solução exata nos instantes de tempo 0.15, 0.30, 0.45. Na segunda linha aparecem os módulos dos coeficientes de Fourier em escala logarítmica, e na última linha os módulos dos coeficientes de ondaletas harmônicas também em escala logarítmica. 

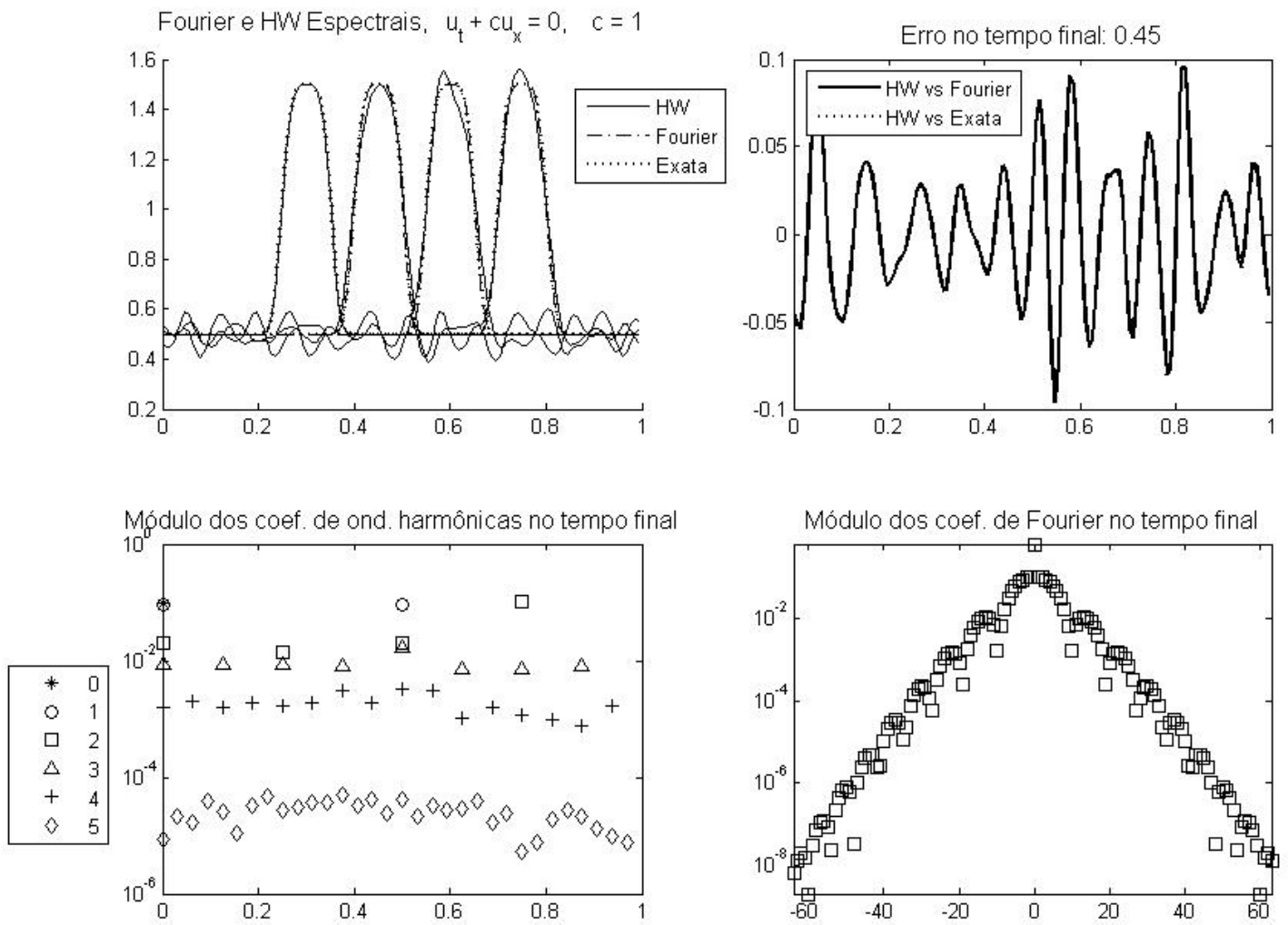

Parâmetros: $d t / d x=0.125 \mathrm{dt}=0.00097656 \quad \mathrm{~N}=128 \quad$ Niveis $\log 2(\mathrm{~N})=7$ Gráfico a cada 0.15 Cortes: Linear $(\mathrm{d})=1$ Näo Linear $(\mathrm{dnl})=0$ Freq. $=0$ Geral $(\mathrm{rc})=0$

Figura 4.3: Adveç̧ão de função exponencial com ondaletas harmônicas considerando $\mathrm{N}=128$ pontos de malha e localidade do suporte $(\mathrm{d}=1)$. No canto superior esquerdo aparecem as soluções exata e aproximadas pelo método de Fourier e de ondaletas harmônicas (HW) em 4 instantes de tempo (0, 0.15, 0.3, 0.45), e no lado direito aparecem as diferenças entre a solução por ondaletas harmônicas (HW) e a solução exata, e em relação à Fourier no tempo final (.45). Na parte inferior estão os módulos dos coeficientes de ondaletas harmônicas por níveis (esquerda) e Fourier (direita) no tempo final (.45) 

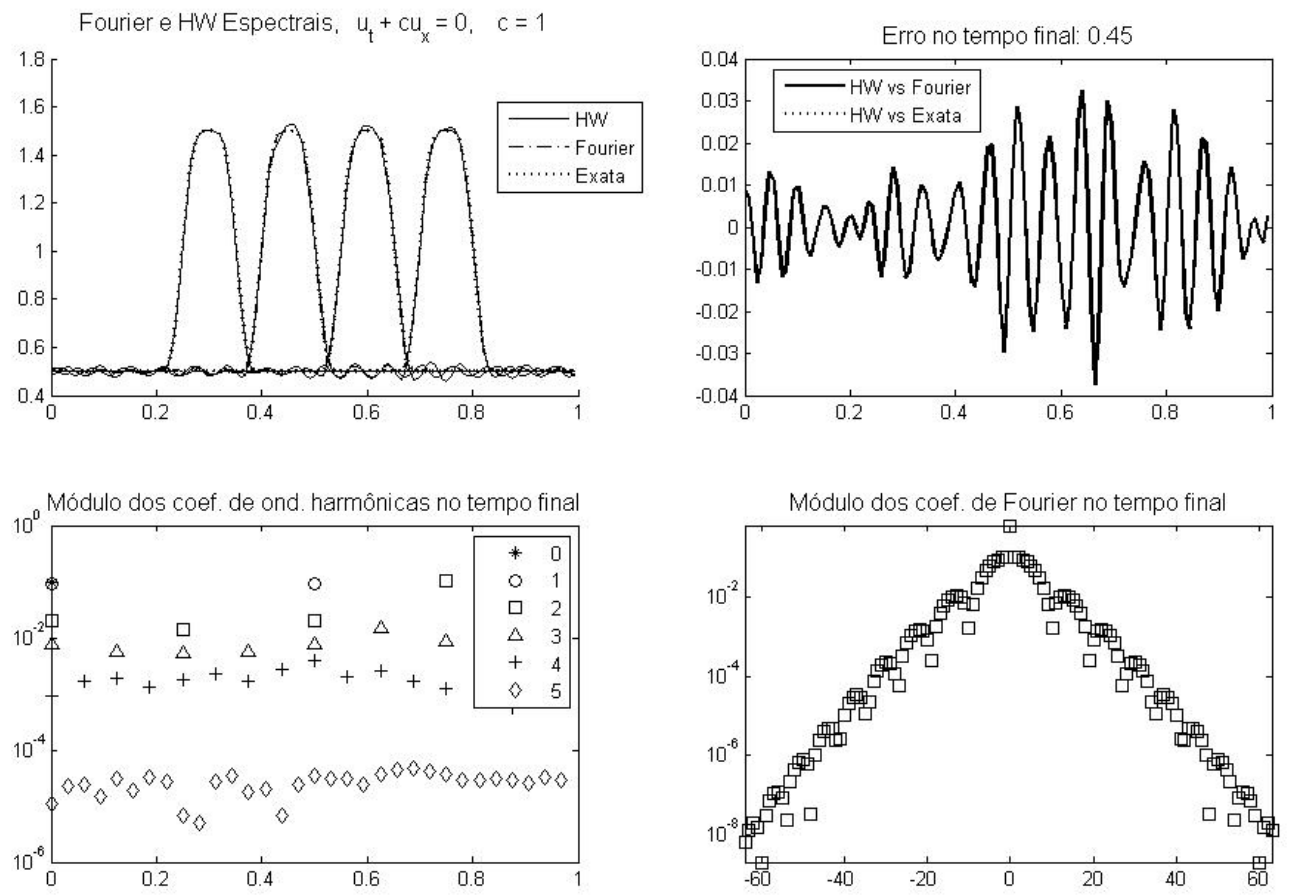

Parâmetros: $d t / d x=0.125 \quad \mathrm{dt}=0.00097656 \quad \mathrm{~N}=128 \quad$ Niveis $\log 2(\mathrm{~N})=7 \quad$ Gráfico a cada 0.15 Cortes: Linear $(\mathrm{d})=3$ Não Linear $(\mathrm{dnl})=0$ Freq. $=0$ Geral $(\mathrm{rc})=0$

Figura 4.4: Advecção de função exponencial com ondaletas harmônicas considerando $\mathrm{N}=128$ pontos de malha e localidade do suporte $(\mathrm{d}=3)$. No canto superior esquerdo aparecem as soluções exata e aproximadas pelo método de Fourier e de ondaletas harmônicas (HW) em 4 instantes de tempo (0, 0.15, 0.3, 0.45), e no lado direito aparecem as diferenças entre a solução por ondaletas harmônicas (HW) e a solução exata, e em relação à Fourier no tempo final (.45). Na parte inferior estão os módulos dos coeficientes de ondaletas harmônicas por níveis (esquerda) e Fourier (direita) no tempo final (.45) 

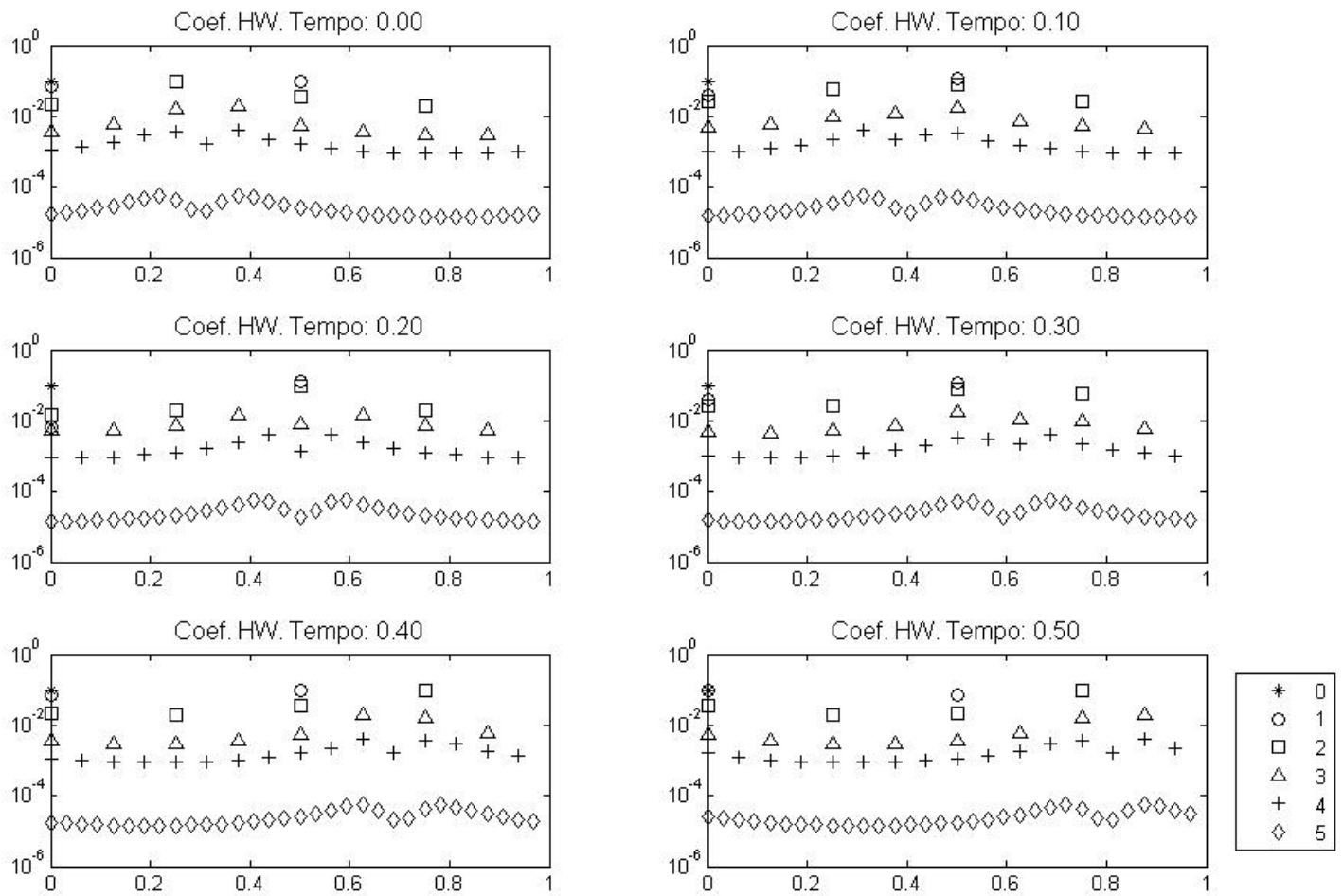

Advecção Linear: $d t / d x=0.0625 \mathrm{~N}=128$ Niveis $\log 2(\mathrm{~N})=7$ Cortes: Linear $(\mathrm{d})=0$ Năo Linear $(\mathrm{dnl})=0$ Freq. $=0$ Geral $(\mathrm{rc})=0$

Figura 4.5: Coeficientes de ondaletas harmônicas para a advecção de função exponencial com ondaletas harmônicas considerando $\mathrm{N}=128$ pontos de malha ao longo do tempo. 
Nos atentaremos agora ao custo computacional envolvido na resolução deste problema utilizando ondaletas harmônicas como base para o método de Galerkin. Executamos o algoritmo, com condição inicial dada pela função exponencial, para diversos valores de $\mathrm{N}$, e consideramos as médias de 5 execuções até o tempo de 0.3 para cada valor de N. Na tabela (4.1) apresentamos os tempos médios de execução, bem como as razões entre os tempos de execução do caso $\mathrm{N}$ em relação ao caso N/2. Vale ressaltar que ao duplicarmos o valor de $\mathrm{N}$ duplicamos também o número de passos de tempo, pois definimos $\Delta t$ como função de $\Delta x$. Nota-se, na tabela em questão, que quando não utilizamos a propriedade de localidade das ondaletas harmônicas temos um aumento de mais de 4 vezes a cada vez que dobramos o valor de $\mathrm{N}$, podendo chegar próximo de 8 vezes maior para cada vez que dobramos o valor de N. Isso mostra que apesar da esparsidade o algoritmo é bastante custoso, tendo ordem de complexidade computacional total próxima de $O\left(N^{3}\right)$, que era de se esperar. Porém, quando utilizamos a localidade do suporte das ondaletas harmônicas temos que no máximo quadruplicamos o tempo computacional a cada vez que dobramos o valor de $\mathrm{N}$, em acordo com o custo computacional total de $O\left(N^{2}\right)$, que é equivalente ao custo do método fundamentado na base de Fourier. Na mesma tabela 4.1 mostramos os erros associados à resolução deste mesmo problema. Percebe-se que a redução do erro é pequena quando comparado com o aumento do custo computacional, principalmente nos casos onde usamos a propriedade de localidade das ondaletas harmônicas. No caso $d=1$ deixa de ser vantajoso usar mais pontos de grade, pois não há redução do erro. Nos casos sem cortes por localidade percebe-se um rápido decaimento do erro, ficando limitado pela ordem de convergência do método de discretização temporal (Runge-Kutta de quarta ordem). 


\begin{tabular}{|c|c|c|c|c|c|c|c|c|}
\hline & \multicolumn{4}{|c|}{$\overline{\text { Tempo (seg) }}$} & \multicolumn{4}{|c|}{ "Erro (norma 2) } \\
\hline $\mathbf{N}$ & $d=0$ & $d=1$ & $d=3$ & Fourier & $\mathrm{d}=\mathbf{0}$ & $d=1$ & $d=3$ & Fourier \\
\hline 16 & 0,03 & 0,02 & 0,02 & 0,01 & $1,20 \mathrm{E}-04$ & $1,20 \mathrm{E}-04$ & $1,20 \mathrm{E}-04$ & $1,20 \mathrm{E}-04$ \\
\hline 32 & 0,09 & 0,07 & 0,09 & 0,01 & $1,31 \mathrm{E}-02$ & $5,18 \mathrm{E}-02$ & $1,31 \mathrm{E}-02$ & $1,31 \mathrm{E}-02$ \\
\hline 64 & 0,48 & 0,25 & 0,35 & 0,02 & $3,47 \mathrm{E}-04$ & 5,39E-02 & $1,68 \mathrm{E}-02$ & $3,47 \mathrm{E}-04$ \\
\hline 128 & 3,07 & 0,95 & 1,41 & 0,06 & $7,66 \mathrm{E}-07$ & $5,41 \mathrm{E}-02$ & $1,66 \mathrm{E}-02$ & $7,66 \mathrm{E}-07$ \\
\hline 256 & 22,51 & 3,64 & 5,44 & 0,16 & $4,77 \mathrm{E}-08$ & $5,42 \mathrm{E}-02$ & $1,65 \mathrm{E}-02$ & $4,77 \mathrm{E}-08$ \\
\hline \multirow[t]{2}{*}{512} & 169,92 & 14,10 & 22,01 & 0,46 & $2,98 \mathrm{E}-09$ & $5,43 \mathrm{E}-02$ & $1,64 \mathrm{E}-02$ & $2,98 \mathrm{E}-09$ \\
\hline & \multicolumn{4}{|c|}{ Razões } & \multicolumn{4}{|c|}{ Razões } \\
\hline $\mathbf{N}$ & $d=0$ & $d=1$ & $d=3$ & Fourier & $d=0$ & $d=1$ & $\mathrm{~d}=\mathbf{3}$ & Fourier \\
\hline $32 / 16$ & 3,47 & 3,11 & 3,97 & 1,44 & 0,01 & 0,00 & 0,01 & 0,01 \\
\hline $64 / 32$ & 5,31 & 3,42 & 3,81 & 2,11 & 37,89 & 0,96 & 0,78 & 37,89 \\
\hline $128 / 64$ & 6,38 & 3,74 & 4,04 & 2,40 & 452,98 & 1,00 & 1,01 & 452,98 \\
\hline $256 / 128$ & 7,33 & 3,83 & 3,86 & 2,69 & 16,06 & 1,00 & 1,01 & 16,06 \\
\hline $512 / 256$ & 7,55 & 3,88 & 4,05 & 2,94 & 16,03 & 1,00 & 1,00 & 16,03 \\
\hline
\end{tabular}

Tabela 4.1: Tempo de execução, em segundos, do algoritmo para resolução da equação do transporte por ondaletas harmônicas, considerando a média de 5 repetições com condição inicial exponencial e até o tempo de 0.30. Na parte inferior aparecem as razões entre os tempos com $\mathrm{N}$ pontos de malha e com $\mathrm{N} / 2$ pontos de malha. Nas colunas à direita aparecem os erros no instante de tempo final, em média de 5 execuções, associados à resolução deste mesmo problema. Os erros foram calculados usando a norma 2 (dividida por $\sqrt{N}$ para normalização). Abaixo dos erros aparecem as razões entre os erros. 


\subsection{Advecção-Difusão Não Linear}

Vamos considerar agora um caso não linear, da equação conhecida por equação de Burgers,

$$
\begin{cases}D(u(x, t))=\frac{\partial u(x, t)}{\partial t}+u(x, t) \frac{\partial u(x, t)}{\partial x}-\nu \frac{\partial^{2} u(x, t)}{\partial x^{2}}=0, & x \in(0,1), \\ u(x, 0)=f(x), & x \in[0,1] \\ u(0, t)=u(1, t), & t \geq 0,\end{cases}
$$

onde $f(x) \in L^{2}([0,1])$ e $\nu>0$. A equação de Burgers pode ser vista como uma boa aproximação do escoamento de fluidos em uma dimensão espacial. Este problema é interessante pois podem surgir fortes gradientes na solução, aproximando-se de uma descontinuidade, quando $\nu \rightarrow 0$. Nem sempre é possível obter solução analítica para este problema. Portanto, soluções numéricas são de grande interesse. Note que na equação 4.12 estamos considerando condições periódicas de fronteira.

Para resolução do problema 4.12 pelo método de Galerkin com base de Fourier precisamos resolver o sistema de equações diferenciais ordinárias (EDOs),

$$
\left\{\begin{array}{l}
\frac{\partial a_{\lambda}(t)}{\partial t}=-\sum_{k+w=\lambda}\left(2 \pi i k a_{w}(t) a_{k}(t)\right)+\nu(2 \pi i \lambda)^{2} a_{\lambda}(t), \\
a_{\lambda}(0)=b_{\lambda}, \quad \lambda=-N / 2, \ldots, N / 2-1
\end{array} .\right.
$$

onde os coeficientes $a$ e $b$ referem-se às expansões em Fourier da aproximação da solução $u_{N}$ e da condição inicial $f_{N}$,

$$
u_{N}(x, t)=\sum_{w=-N / 2}^{N / 2-1} a_{w}(t) e^{2 \pi i w x}
$$

e

$$
f_{N}(x)=\sum_{k=-N / 2}^{N / 2-1} b_{k} e^{2 \pi i k x}
$$

Na resolução pelo método de Galerkin com base de ondaletas harmônicas precisa-se resolver o 
sistema de EDOs,

$$
\begin{cases}\frac{d a_{0}(t)}{d t} & =0 \\ \frac{d a_{2^{r}+s}(t)}{d t} & =-a_{0}(t) \sum_{q=0}^{2^{r}-1} a_{2^{r}+q}(t) \gamma_{q s}^{r} \\ & -\sum_{j=0}^{n-2} \sum_{k=0}^{2^{j}-1} \sum_{p=0}^{n-2} \sum_{q=0}^{2^{p}-1}\left(a_{2^{j}+k}(t) a_{2^{p}+q}(t) P(0)_{k q s}^{j p r}+a_{2^{j}+k}^{*}(t) a_{2^{p}+q}(t) P(1)_{k q s}^{j p r}\right) \\ & -\sum_{j=0}^{n-2} \sum_{k=0}^{2^{j}-1} \sum_{p=0}^{n-2} \sum_{q=0}^{2^{p}-1}\left(a_{2^{j}+k}(t) a_{2^{p}+q}^{*}(t) P(2)_{k q s}^{j p r}\right) \\ & +\nu \sum_{k=0}^{2^{r}-1} a_{2^{r}+k}(t) \chi_{k s}^{r} \\ & \operatorname{para} r=0,1, \ldots, n-2, \quad s=0,1, \ldots, 2^{r}-1, \\ \frac{d a_{N / 2}(t)}{d t} \quad & +N \pi i a_{0}(t) a_{N / 2}(t) \\ & -\sum_{j=0}^{n-2} \sum_{k=0}^{2^{j}-1} \sum_{p=0}^{n-2} \sum_{q=0}^{2^{p}-1}\left(a_{2^{j}+k}^{*}(t) a_{2^{p}+q}^{*}(t) N_{k q}^{j p *}\right) \\ & -\nu(N \pi)^{2} a_{N / 2}(t) .\end{cases}
$$

sujeito à condição inicial dada por

$$
a_{w}(0)=b_{w}, \quad w=0, \ldots, N / 2
$$

onde agora os coeficientes $a$ e $b$ referem-se as expansões da aproximação da solução, $u_{N}$, e da condição inicial aproximada, $f_{N}$, na base de ondaletas harmônicas,

$$
u_{N}(x, t)=a_{0}(t)+\sum_{j=0}^{n-2} \sum_{k=0}^{2^{j}-1}\left(a_{2^{j}+k}(t) \psi_{j, k}(x)+a_{2^{j}+k}^{*}(t) \psi_{j, k}^{*}(x)\right)+a_{N / 2}(t) \psi_{n-1}(x)
$$

e

$$
f_{N}(x)=b_{0}+\sum_{j=0}^{n-2} \sum_{k=0}^{2^{j}-1}\left(b_{2^{j}+k} \psi_{j, k}(x)+b_{2^{j}+k}^{*} \psi_{j, k}^{*}(x)\right)+b_{N / 2} \psi_{n-1}(x) .
$$

\subsubsection{Comparativo com Muniandy e Moroz (1997)}

O primeiro experimento numérico que vamos fazer é baseado no trabalho de Muniandy e Moroz (1997). No exemplo teste de Muniandy e Moroz (1997) é proposta a resolução da equação de Burgers com condição inicial dada por $f(x)=\sin (2 \pi x)$, considerando coeficiente de viscosidade $\nu=0.025$ e utilizando 128 pontos de malha (que é equivalente a termos $n=7, N=2^{7}=128$ ). Porém

o método que desenvolvemos ao longo desta dissertação difere do método de Muniandy e Moroz (1997) que só considera na base de ondaletas harmônicas os elementos $\psi_{j, k}(x)$ para $j=0,1, \ldots, n-2$ 
e $k=0,1, \ldots, 2^{j}-1$, isto é, ficando a expansão de $u_{N}$ da seguinte forma,

$$
u_{N}(x, t)=\sum_{j=0}^{n-2} \sum_{k=0}^{2^{j}-1} a_{2^{j}+k}(t) \psi_{j, k}(x) .
$$

Como visto na seção dedicada às ondaletas harmônicas, sabemos que ao nos restringirmos apenas a estes elementos na base estaremos descartando números de onda relevantes, além de estarmos limitados a aproximações de soluções com média zero (integral nula no intervalo unitário), já que não há ondaleta pai, ou função escala, na base. Por outro lado, esta simplificação reduz o número de coeficientes de conexão não lineares a serem calculados. Infelizmente no trabalho original de Muniandy e Moroz (1997) não há detalhes quanto ao método numérico usado para a resolução do sistema de EDOs. Reproduzimos na figura 4.6 seus resultados, onde aparecem instantes de tempo da evolução da onda senoidal, mas não é dito quais são estes instantes de tempo.

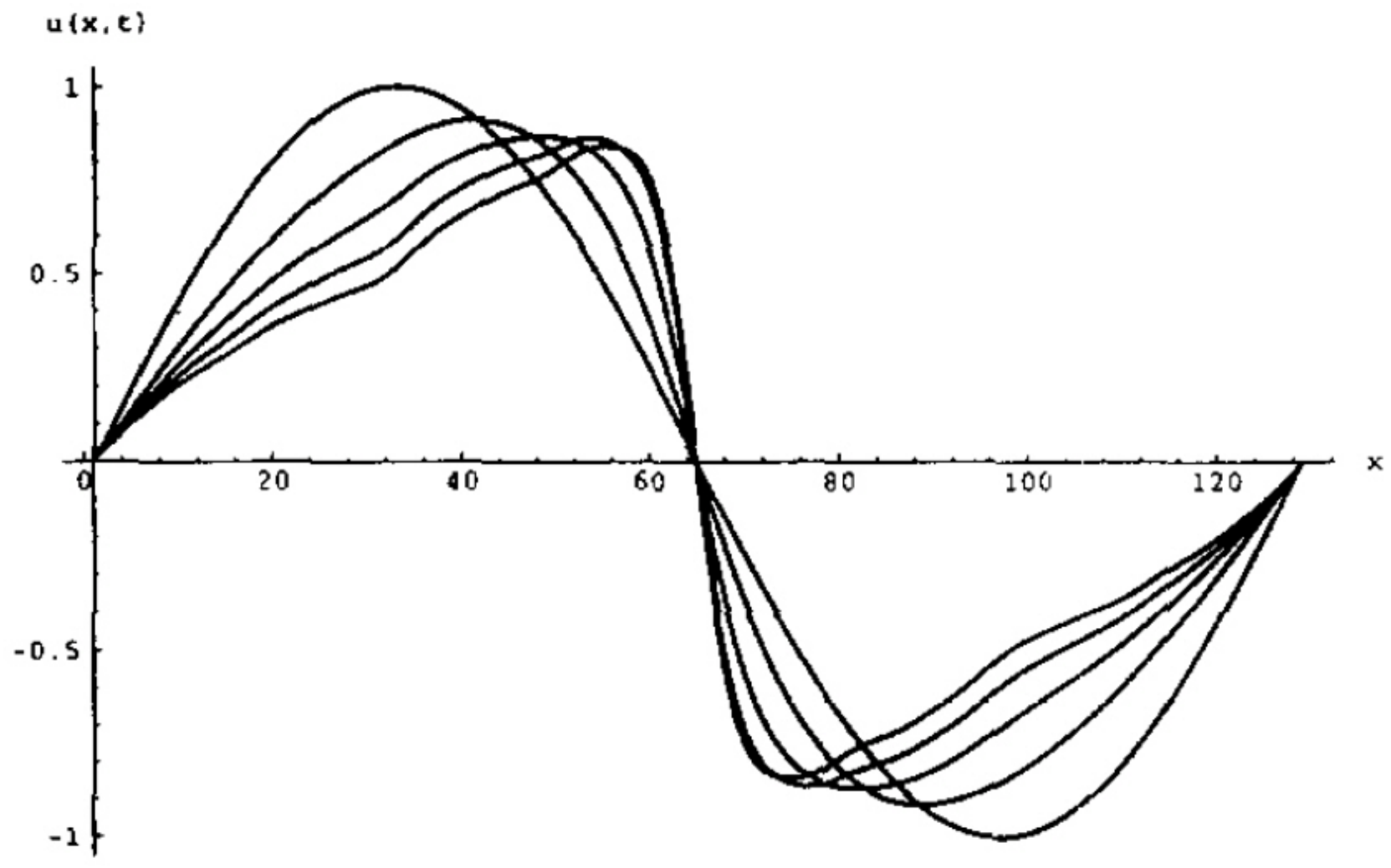

Figura 4.6: Solução numérica da equação de Burgers obtida em Muniandy e Moroz (1997) considerando $\nu=0.025, N=128$ para a condição inicial senoidal

Para a obtenção dos resultados de Muniandy e Moroz (1997) foram feitas algumas considerações para redução do custo computacional, como a de se utilizar a localidade do suporte das ondaletas 
harmônicas com parâmetro $d=1$. Além disso no artigo são considerados os coeficientes de conexão com diferenças de níveis de no máximo uma unidade, isto é, considera-se que o valor dos coeficientes de conexão não lineares são não nulos apenas a para as tríades $\{j, p, r\}=\{\{r-1, r-1, r\},\{r, r-$ $1, r\},\{r-1, r, r\}\}$. Porém tais considerações foram levadas em conta apenas para os níveis maiores que 3, ou seja, para valores de $r$ abaixo de 4 são calculados todos os coeficientes de conexão não lineares e utilizando-se todo o suporte das ondaletas harmônicas.

Simulamos as mesmas condições utilizando-se a base completa de ondaletas harmônicas e um método de Runge-Kutta de quarta ordem para a discretização temporal do sistema de EDOs, com $\Delta t=\Delta x / 16$ para manter estabilidade numérica do método. Mostramos na figura 4.7 os resultados, onde usamos a propriedade de localidade de suporte de ondaletas harmônicas tanto nos coeficientes lineares como nos não lineares. Apresentamos os gráficos a cada 0.05 unidades de tempo, até o tempo final 0.3 . 

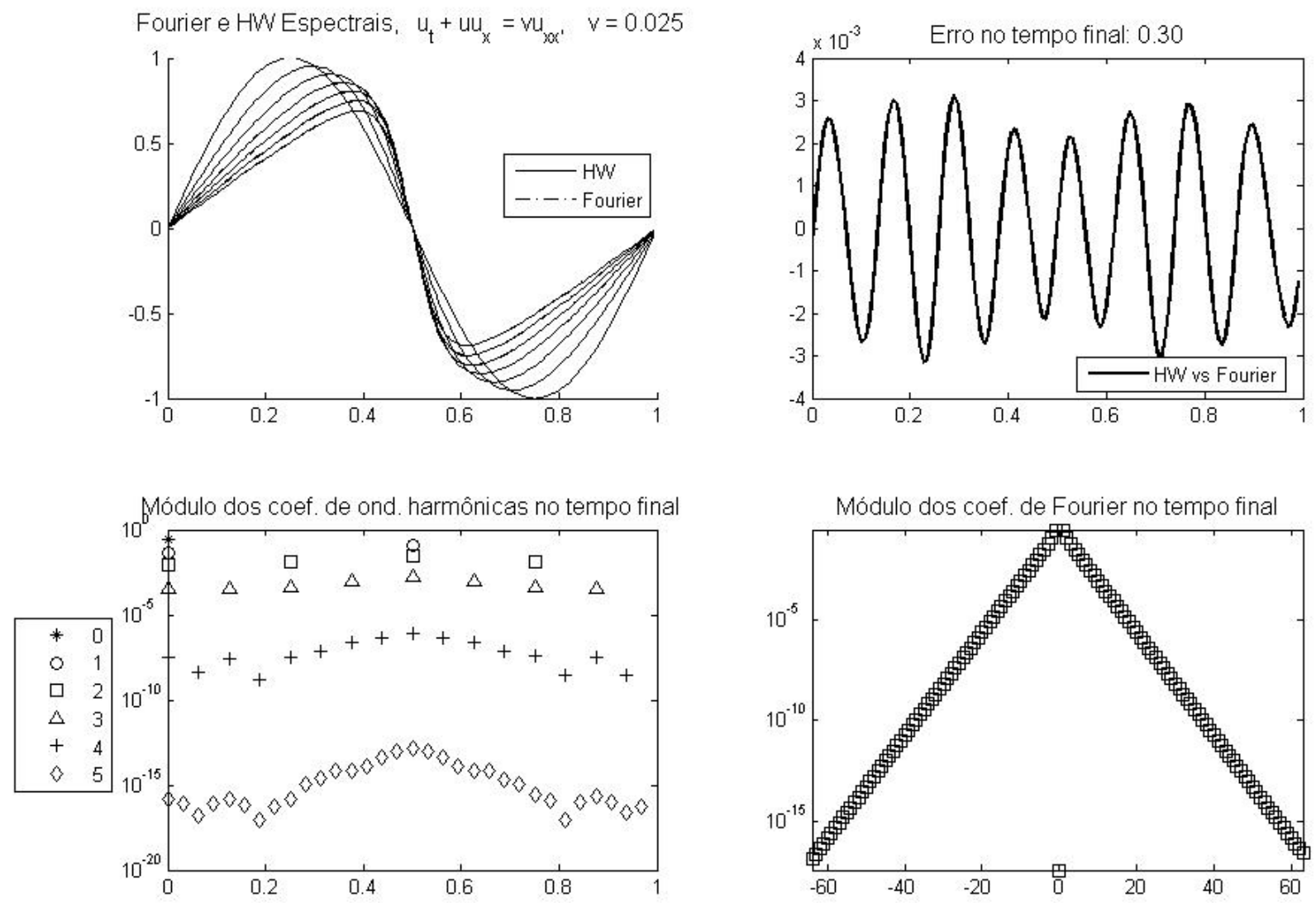

$\mathrm{dt} / \mathrm{d} \mathrm{x}=0.0625 \mathrm{dt}=0.00048 \mathrm{~N}=128$ Niveis $\mathrm{n}=7$ Gráfico a cada 0.05 Cortes: Linear $\mathrm{d}=1$ Näo Linear $\mathrm{d}=1 \mathrm{Freq} .=1 \mathrm{Geral}(\mathrm{rc})=3$

Figura 4.7: Solução numérica da equação de Burgers considerando $\nu=0.025, N=128$, para a condição inicial senoidal com a base completa de ondaletas harmônicas. No canto superior esquerdo aparecem as soluções numéricas por ondaletas harmônicas (HW) e por Fourier a cada 0.05 unidades de tempo, e no canto superior direito a diferença entre os métodos para o tempo final 0.3. Na parte inferior aparecem os módulos dos coeficientes ondaletas harmônicas (por níveis) e de Fourier (por número de onda) para o tempo final em escalas logarítmicas. A solução considera cortes por localidade da base $(d=1)$ e cortes por frequência $(\xi=1)$. Os cortes foram feitos apenas em níveis maiores que 3 .

A solução apresentada na figura 4.7 difere da solução obtida em Muniandy e Moroz (1997), apresentada na figura 4.6, por não ter oscilações perto da descontinuidade, mas infelizmente, por não termos mais informações sobre a discretização temporal e em quais instantes de tempo a solução de Muniandy e Moroz (1997) é considerada, não é possível fazermos uma comparação mais detalhada. Além disso, aparentemente não há razão para considerarmos as simplificações apenas para níveis maiores que 3, que na verdade seria uma escolha arbitrária dependente da condição inicial e viscosidade de cada problema. Se simularmos sem utilizar cortes referentes as relações entre as frequências, com corte por localidade com parâmetro $d=1$, e sendo essas simplificações para todos os níveis, vamos obter os resultados apresentados na figura 4.8. Nota-se um pequeno 
aumento de oscilações, mas muito pouco considerando os resultados obtidos por Muniandy e Moroz (1997), destacados na figura 4.6. Analisamos também o caso onde são realizados cortes apenas de localidade, com parâmetro $d=3$, para todos os níveis. Aproveitamos este teste para incluir um comparativo com um método de diferenças finitas, Lax-Wendroff, com os mesmos valores de $\Delta t$ e $\Delta x$. Apresentamos os resultados na figura 4.9. Nota-se que a escolha do parâmetro de corte $d=3$ trás um resultado com menos ruídos que no caso onde $d=1$.
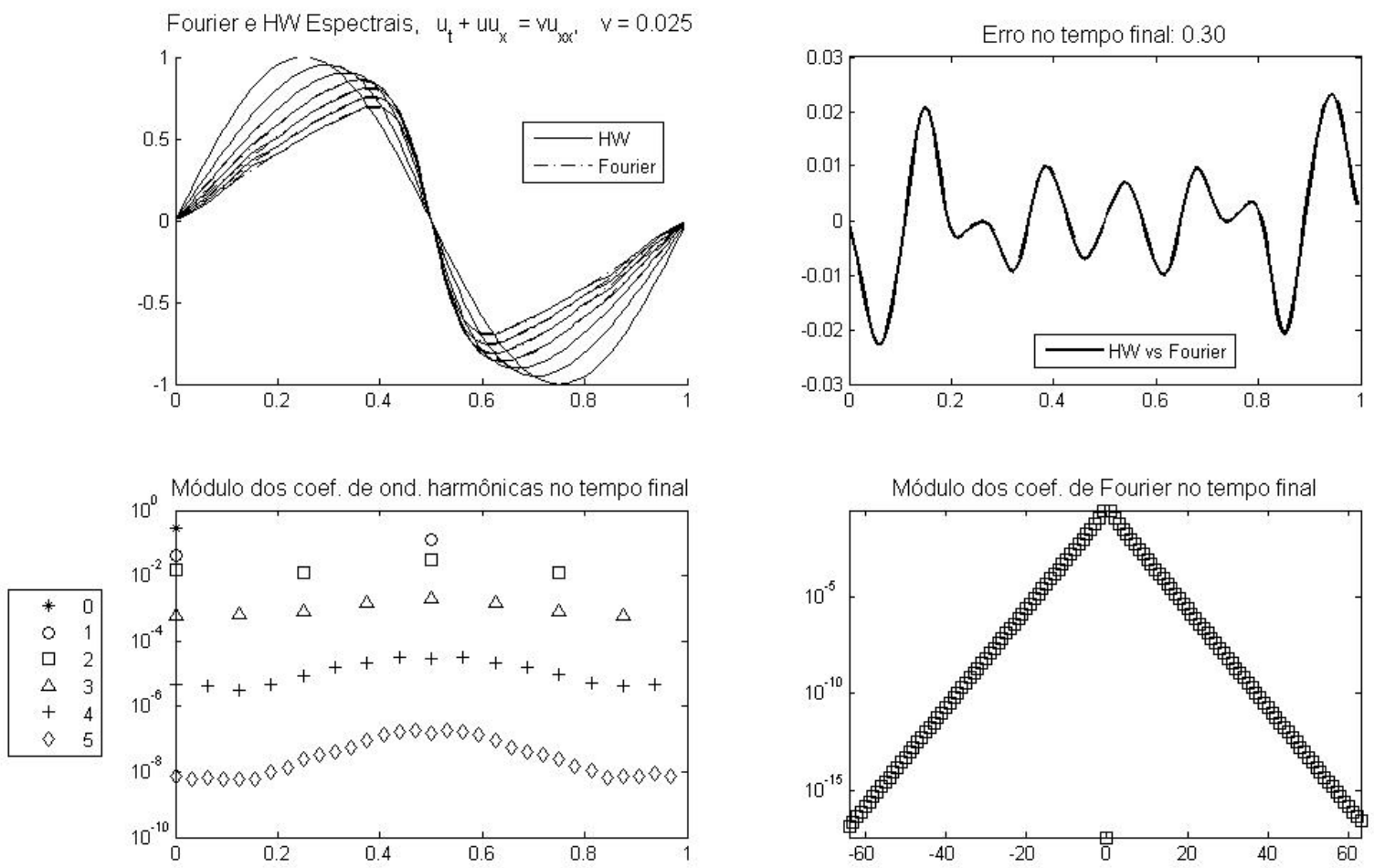

$d t / d x=0.0625 d t=0.00048828 \quad N=128 \quad$ Niveis $\log 2(N)=7$ Gráfico a cada 0.05

Cortes: Linear $d=1$ Não Linear $d=1$ Freq. $=0$ Geral $(\mathrm{rc})=0$

Figura 4.8: Solução numérica da equação de Burgers considerando $\nu=0.025, N=128$ para a condição inicial senoidal com a base completa de ondaletas harmônicas. No canto superior esquerdo aparecem as soluções numéricas por ondaletas harmônicas (HW) e por Fourier a cada 0.05 unidades de tempo, e no canto superior direito a diferença entre os métodos para o tempo final 0.3. Na parte inferior aparecem os módulos dos coeficientes ondaletas harmônicas (por níveis) e de Fourier (por números de onda) para o tempo final em escalas logarítmicas. A solução considera cortes por localidade da base $(d=1)$ mas não considera cortes por frequência. Os cortes foram feitos em todos os níveis. 

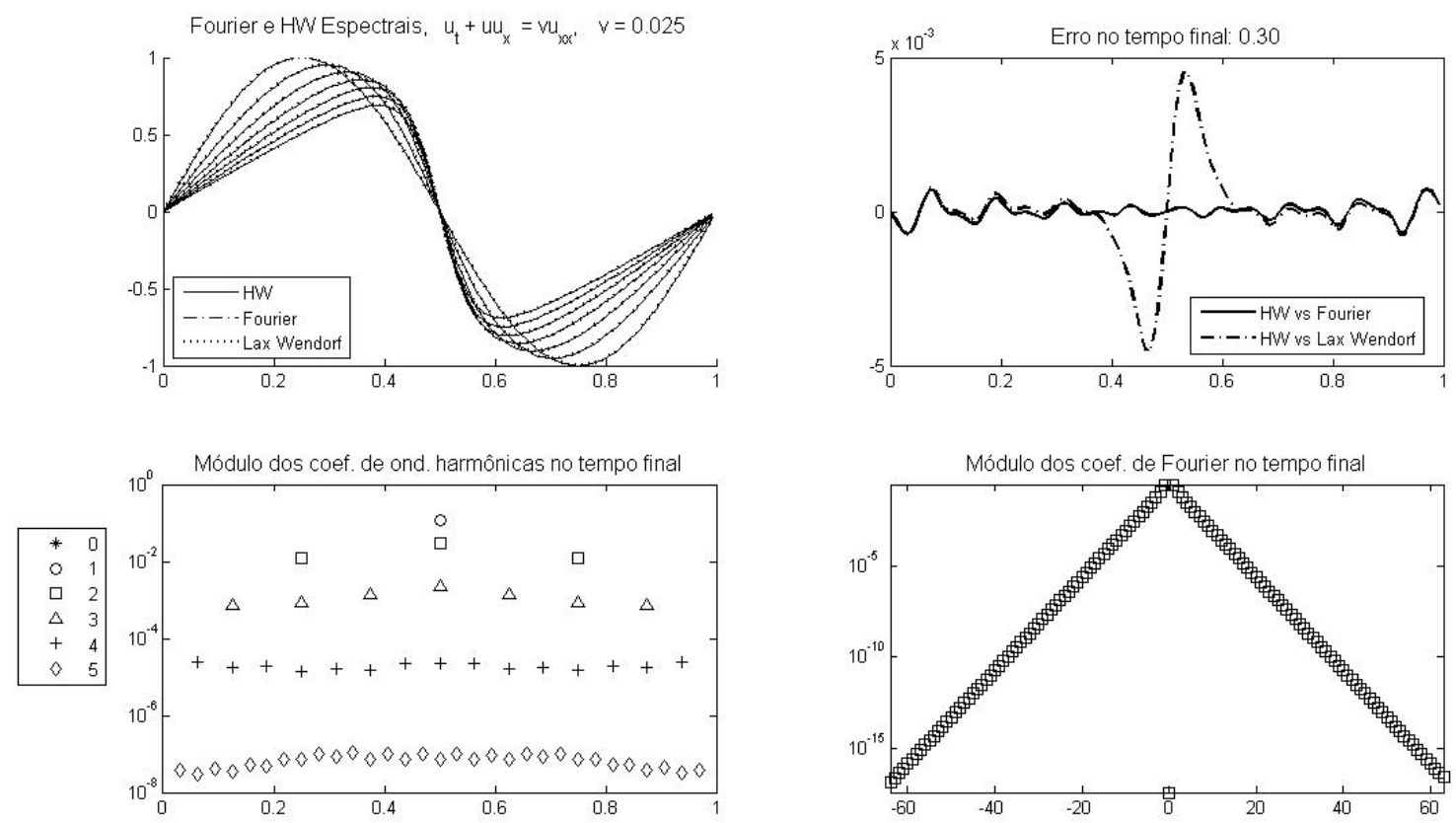

$\mathrm{dt} / \mathrm{dx}=0.0625 \mathrm{dt}=0.00048828 \mathrm{~N}=128$ Niveis $\mathrm{n}=7$ Gráfico a cada 0.05 Cortes: Linear $\mathrm{d}=3$ Não Linear $\mathrm{d}=3 \mathrm{Freq} .=0 \mathrm{Geral}(\mathrm{rc})=0$

Figura 4.9: Solução numérica da equação de Burgers considerando $\nu=0.025, N=128$ para a condição inicial senoidal com a base completa de ondaletas harmônicas. No canto superior esquerdo aparecem as soluções numéricas por ondaletas harmônicas (HW), por Fourier e por Lax-Wendroff a cada 0.05 unidades de tempo, e no canto superior direito a diferença entre os métodos para o tempo final 0.3. Na parte inferior aparecem os módulos dos coeficientes ondaletas harmônicas (por níveis) e de Fourier (por números de onda) para o tempo final em escalas logarítmicas. A solução considera cortes por localidade da base $(d=3)$ mas não considera cortes por frequência. Os cortes foram feitos em todos os níveis. 


\subsubsection{Custo computacional}

Vamos olhar outras simulações numéricas com ondaletas harmônicas para a equação de Burgers, começando por olhar a influência do uso de localidade do suporte da base de ondaletas harmônicas. Resolvemos a equação com viscosidade $\nu=0.003$ e $N=64$ pontos de malha, considerando a condição inicial exponencial (4.3) com condições de localidade de suporte de ondaletas harmônicas, $d$, variando entre zero e três, onde zero quer dizer que usamos as ondaletas sem restrição de suporte. Na figura 4.10 apresentamos os casos $d=0,1,3$ a cada 0.1 unidades de tempos, com $\Delta t=\Delta x / 16$ e novamente um método Runge-Kutta de quarta ordem para discretização temporal. Nota-se que a forma do sinal é essencialmente preservada, porém pequenas oscilações podem aparecer.
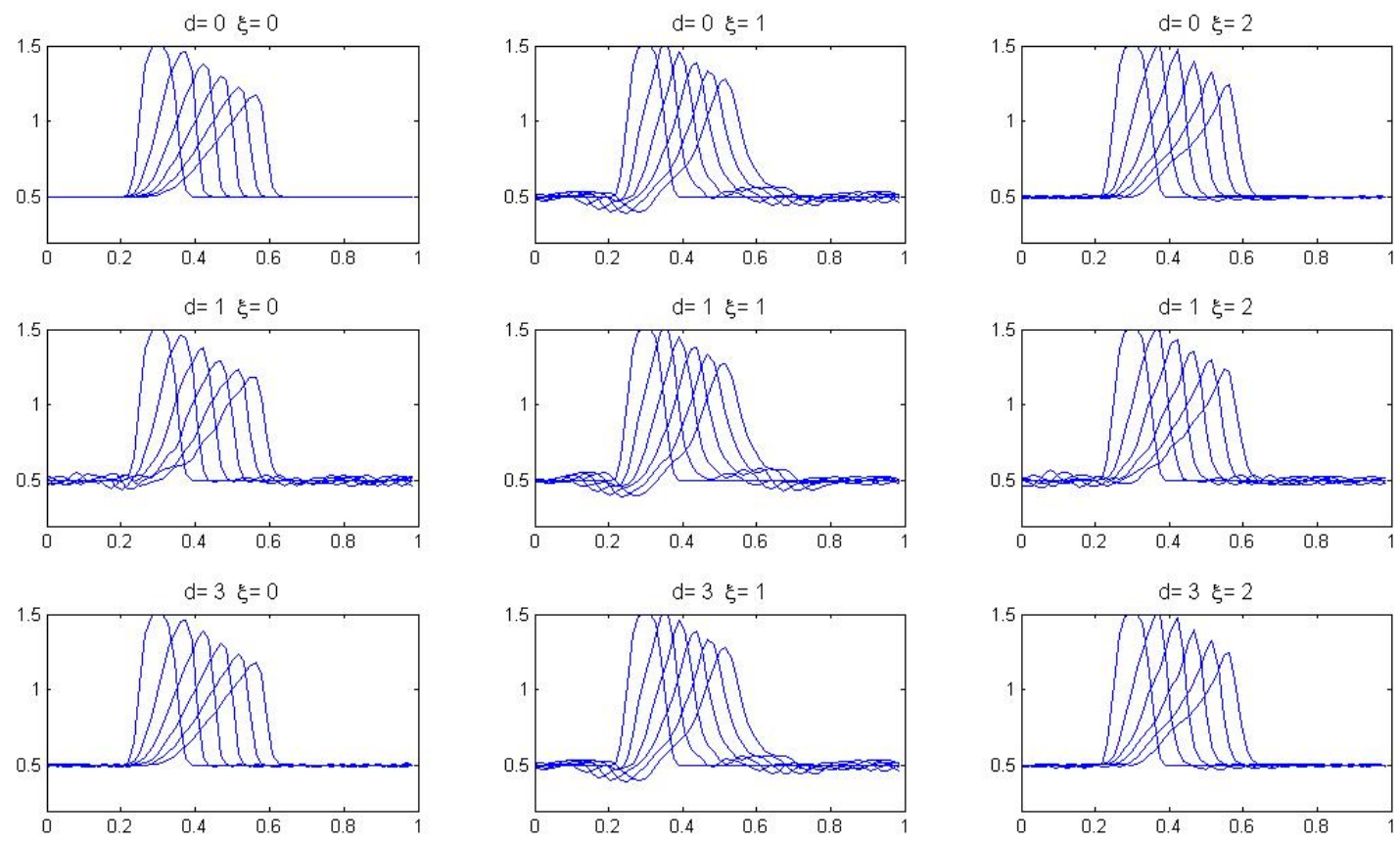

Parâmetros: $\quad \mathrm{dt} / \mathrm{d} \mathrm{x}=0.0625 \mathrm{dt}=0.00097656 \quad \mathrm{~N}=64 \quad$ Niveis $\log 2(\mathrm{~N})=6$

Figura 4.10: Solução numérica da equação de Burgers considerando $\nu=0.003, N=64$ para a condição inicial exponencial com a base completa de ondaletas harmônicas usando a localidade do suporte de ondaletas harmônicas e uma região de vizinhança de interação entre frequências com parâmetro $\xi$. Os gráficos aparecem a cada 0.1 unidades de tempo, começando pela condição inicial.

Em contrapartida, para esta mesma simulação, o custo computacional cai muito quando se restringe o suporte das ondaletas harmônicas. Apresentamos na tabela 4.2 os tempos, em segundos, da execução do algoritmo com ondaletas harmônicas para o caso que acabamos de ilustrar para diversos valores de $N$ até o instante de tempo final igual a 0.2. Nesta tabela percebe-se que ao não utilizar a propriedade de localidade do suporte da base ficamos com um algoritmo com $O\left(N^{4}\right)$, 


\begin{tabular}{|cccccccc|}
\hline \multicolumn{7}{|c|}{ Tempo Médio em segundos } \\
\hline $\mathbf{N}$ & $d=0$ & $d=1$ & $d=3$ & $d=3, \xi=1$ & $d=3, \xi=2$ & PS $(d=0)$ & PS $(d=3)$ \\
\hline $\mathbf{1 6}$ & 0,19 & 0,09 & 0,09 & 0,16 & 0,12 & 0,11 & 0,11 \\
\hline $\mathbf{3 2}$ & 0,69 & 0,44 & 0,61 & 0,54 & 0,66 & 0,28 & 0,28 \\
\hline $\mathbf{6 4}$ & 7,76 & 2,37 & 5,05 & 2,74 & 4,10 & 0,87 & 0,77 \\
\hline $\mathbf{1 2 8}$ & 114,01 & 15,24 & 40,04 & 13,36 & 25,89 & 3,41 & 2,25 \\
\hline $\mathbf{2 5 6}$ & 1804,80 & 103,46 & 298,85 & 65,51 & 131,03 & 18,28 & 7,03 \\
\hline \multicolumn{7}{c}{} & \multicolumn{7}{c}{ Razão entre os tempos } & & \\
\hline$N$ / $\left(\frac{N}{2}\right)$ & $d=0$ & $d=1$ & $d=3$ & $d=3, \xi=1$ & $d=3, \xi=2$ & PS $(d=0)$ & PS $(d=3)$ \\
\hline $\mathbf{3 2} / \mathbf{1 6}$ & 3,59 & 5,06 & 6,92 & 3,45 & 5,69 & 2,57 & 2,49 \\
\hline $\mathbf{6 4} / \mathbf{3 2}$ & 11,17 & 5,37 & 8,34 & 5,05 & 6,21 & 3,07 & 2,75 \\
\hline $\mathbf{1 2 8} / \mathbf{6 4}$ & 14,68 & 6,43 & 7,92 & 4,88 & 6,31 & 3,92 & 2,93 \\
\hline $\mathbf{2 5 6} / \mathbf{1 2 8}$ & 15,83 & 6,79 & 7,46 & 4,90 & 5,06 & 5,36 & 3,13 \\
\hline
\end{tabular}

Tabela 4.2: Tempo de execução do algoritmo para resolução da equação de Burgers por ondaletas harmônicas, considerando a média de 5 repetições com condição inicial exponencial, $\Delta t=\frac{\Delta x}{16}, \nu=0.003$ e tempo final de 0.2. Consideram-se as características de localidade $(d)$ da base tanto nos coeficientes de conexão lineares quanto nos não lineares. As características de interações entre frequências vizinhas $(\xi)$ foi considerado para os coeficientes não lineares apenas. As duas últimas colunas apresentam os tempos utilizando um método pseudo-espectral (PS) para ondaletas harmônicas. Na parte inferior aparecem as razões entre os tempos com $\mathrm{N}$ pontos de malha e com $\mathrm{N} / 2$ pontos de malha.

levando em conta que estamos dando $N$ vezes mais passos no tempo, ficamos com um algoritmo para a execução da parte de discretização espacial da ordem de $O\left(N^{3}\right)$, como era de se esperar. Porém, ao usarmos restrições sobre o suporte das ondaletas, com $d=1,3$, obtém-se um custo computacional da parte de discretização espacial, retirando-se o custo da discretização temporal, da ordem de $O\left(N^{2}\right)$. Comparando essas estimativas com o método de Galerkin com base de Fourier, nota-se que o método com ondaletas harmônicas usando a propriedade de localidade tem a mesma ordem de custo computacional de discretização da parte espacial do método fundamentado em Fourier, $O\left(N^{2}\right)$. Porém é mais caro computacionalmente que a abordagem pseudo-espectral, com custo computacional da ordem de $O(N \log N)$ da parte de discretização espacial. Mostramos os tempos de execução para o mesmo problema agora com base de Fourier na tabela 4.3 .

Uma outra abordagem possível é uma simplificação nas interações entre as frequências da parte não linear da equação, comumente usada em modelos de turbulência (EGGERS; GROSSMAN, 1991; BIFERALE, 2003). Esta simplificação consiste basicamente em considerar que uma certa frequência interage apenas com frequências vizinhas, ou próximas, na componente não linear da equação de Burgers. Está fundamentada no que é conhecido por efeito de cascata, onde a energia é transportada somente para escalas (frequências) vizinhas. No trabalho de Muniandy e Moroz (1997) essa simplificação é feita tomando-se os valores dos coeficientes de conexão não lineares não nulos apenas a para as tríades $\{j, p, r\}=\{\{r-1, r-1, r\},\{r, r-1, r\},\{r-1, r, r\}\}$. Um caso geral seria dizer que um coeficiente de conexão não linear é não nulo somente se $j$ e $p$ forem tais que $|r-j| \leq \xi$, $|r-p| \leq \xi$, para um certo parâmetro $\xi$ que indica a vizinhança da frequência $r$ a ser considerada. No caso das ondaletas harmônicas, podemos fazer uma simplificação da seguinte forma. 


\begin{tabular}{|ccc|}
\hline \multicolumn{3}{|c|}{ Tempo Médio em segundos - Fourier } \\
\hline \hline $\mathbf{N}$ & Espectral & Pseudo-Espectral \\
\hline $\mathbf{1 6}$ & 0,10 & 0,06 \\
\hline $\mathbf{3 2}$ & 0,25 & 0,09 \\
\hline $\mathbf{6 4}$ & 1,15 & 0,15 \\
\hline $\mathbf{1 2 8}$ & 7,80 & 0,33 \\
\hline $\mathbf{2 5 6}$ & 58,02 & 0,72 \\
\hline $\mathbf{5 1 2}$ & 444,18 & 1,99 \\
\hline \hline \multicolumn{3}{|c|}{ Razão entre os tempos } \\
\hline$N$ / ( $\left.\frac{N}{2}\right)$ & Espectral & Pseudo-Espectral \\
\hline $\mathbf{3 2} / \mathbf{1 6}$ & 2,48 & 1,59 \\
\hline $\mathbf{6 4} / \mathbf{3 2}$ & 4,66 & 1,75 \\
\hline $\mathbf{1 2 8} / \mathbf{6 4}$ & 6,78 & 2,12 \\
\hline $\mathbf{2 5 6} / \mathbf{1 2 8}$ & 7,43 & 2,23 \\
\hline $\mathbf{5 1 2} / \mathbf{2 5 6}$ & 7,66 & 2,75 \\
\hline
\end{tabular}

Tabela 4.3: Tempo de execução do algoritmo para resolução da equação de Burgers com base de Fourier, considerando a média de 5 repetições com condição inicial exponencial, $\Delta t=\frac{\Delta x}{8}, \nu=0.003$ e tempo final de 0.2. Na parte inferior aparecem as razões entre os tempos com $\mathrm{N}$ pontos de malha e com $\mathrm{N} / 2$ pontos de malha.

- $P(0)_{k q s}^{j p r}$ : Consideram-se nulos os elementos tais que $j<r-\xi, j>r, p<r-\xi, p>r$.

- $P(1)_{k q s}^{j p r}$ : Consideram-se nulos os elementos tais que $j<p-\xi, j>p, r<p-\xi, r>p$.

- $P(2)_{k q s}^{j p r}$ : Consideram-se nulos os elementos tais que $p<j-\xi, p>j, r<j-\xi, r>j$.

Onde podemos escolher $\xi=1,2, \ldots$ como parâmetro que define a vizinhança de interação entre as frequências. Realizamos testes para os valores de $\xi=1,2$ para a equação de Burgers com 64 pontos de malha, $\Delta t=\frac{\Delta x}{16}, \nu=0.003$ e condição inicial exponencial. Mostramos na figura 4.10 as soluções aproximadas nos tempos $0.1,0.2,0.3,0.4$ e 0.5, utilizando ondaletas harmônicas e simplificação por frequência com parâmetro $\xi=1,2$. Nota-se que, ao fazermos uma simplificação destas, eliminando interações entre frequências, e compararmos com as aproximações sem estas simplificações, apresentadas na mesma figura 4.10, as soluções numéricas apresentam oscilações.

Apresentamos nas duas últimas colunas da tabela 4.2 estimativas de custo computacional levando em conta a simplificação por frequências. Verificamos que há uma redução expressiva do custo computacional, deixando a parte de discretização espacial com custo computacional aparentemente com ordem menor que $N^{2}$, mas maior que $N$. Porém obtemos uma aproximação da solução com ruídos e oscilações.

Analisaremos a seguir o custo benefício de se considerar simplificações nos cálculos, usando os parâmetros $d$ e $\xi$, em relação ao erro gerado pelas simplificações. Para tanto vamos considerar um teste da equação de Burgers com condição inicial exponencial, 64 pontos de malha, $\Delta t=\frac{\Delta x}{16}$, viscosidade $\nu=0.003$ até o tempo final de 0.3 , sem cortes como referência $(d=0, \xi=0)$. Utilizando a norma euclidiana, tomamos as variações relativas entre as soluções aproximadas no tempo final 


\begin{tabular}{|cc|cc|}
\hline $\mathbf{d}$ & $\xi$ & Erro & Tempo de Execução \\
\hline 0 & 0 & 0 & 24,1932 \\
0 & 1 & $13,41 \%$ & $-47,54 \%$ \\
0 & 2 & $3,10 \%$ & $-20,83 \%$ \\
1 & 0 & $3,15 \%$ & $-72,23 \%$ \\
1 & 1 & $13,69 \%$ & $-84,57 \%$ \\
1 & 2 & $3,82 \%$ & $-78,50 \%$ \\
3 & 0 & $0,49 \%$ & $-37,99 \%$ \\
3 & 1 & $13,40 \%$ & $-68,40 \%$ \\
3 & 2 & $3,17 \%$ & $-49,14 \%$ \\
\hline
\end{tabular}

Tabela 4.4: Na primeira linha aparece o tempo de execução do algoritmo para resolução da equação de Burgers com ondaletas harmônicas, considerando $N=64, \Delta t=\frac{\Delta x}{16}, \nu=0.003$, tempo final 0.3 , sem uso de localidade $(d=0)$ e sem uso de cortes em frequências $(\xi=0)$. Nas linhas que seguem aparecem os aumentos/reduções do erro relativo (equação (4.21)) e do tempo de execução relativo (equação 4.22) com diversos valores de $d$ e $\xi$.

com parâmetros $d$ e $\xi$, dada por $\tilde{u}_{\xi}^{d}$, e a solução aproximada sem cortes, também no tempo final, $\tilde{u}_{0}^{0}$, como sendo,

$$
\Delta \tilde{u}_{\xi}^{d}=\frac{\left\|\tilde{u}_{\xi}^{d}-\tilde{u}_{0}^{0}\right\|_{2}}{\left\|\tilde{u}_{0}^{0}\right\|} .
$$

Além disso consideramos as variações relativas entre os tempos de execução, $\tau_{\xi}^{d}$, do algoritmo para acharmos a aproximação $\tilde{u}_{\xi}^{d}$,

$$
\Delta \tau_{\xi}^{d}=\frac{\tau_{\xi}^{d}-\tau_{0}^{0}}{\tau_{0}^{0}} .
$$

Mostramos na tabela 4.4 os resultados obtidos. Percebe-se claramente uma redução do tempo de execução ao sermos mais restritivos na escolha dos parâmetros de corte. Nota-se que o uso de cortes em frequências $(\xi>0)$ é responsável por grande parte do erro relativo, sendo que com $\xi=1$ o erro relativo fica próximo de $13 \%$ e com $\xi=2$ próximo de $3 \%$, e com redução de custo computacional pouco significativo. Já o impacto dos cortes por localidade $(d>0)$ no erro relativo é bem menor, e acarretam uma variação de aproximadamente $3 \%$ e $.5 \%$ em cada caso, $d=1$ e $d=3$, e com um bom ganho computacional. Os formatos do sinal com as diversas escolhas dos parâmetros podem ser vistos na figura 4.10 . 


\subsubsection{Métodos de suavização}

No trabalho de Muniandy e Moroz (1997) é feito um tratamento a fim de se reduzir os efeitos oscilatórios que surgiram ao considerarmos as propriedades de localidade e cortes nos níveis para os coeficientes de conexão, onde consideraram uma suavização utilizando aproximações polinomiais. Novamente não há mais detalhes sobre o procedimento de suavização usado, portanto testamos aqui usando alguns possíveis métodos de suavização da solução numérica.

Considere uma função $f$ definida em $N$ pontos $x_{j}$ de uma malha numérica, tal que $f_{j}=f\left(x_{j}\right)$. Então podemos olhar para os seguintes filtros $(\bar{f})$ :

- Filtro Shapiro de ordem $k$ :

$$
\begin{aligned}
f_{j}^{(0)} & =f_{j}, \quad j=1, \ldots, N, \\
f_{j}^{(k)} & =\frac{1}{4}\left(f_{j-1}^{(k-1)}+2 f_{j}^{(k-1)}+f_{j+1}^{(k-1)}\right), \\
\bar{f}_{j} & =f_{j}^{(k)} .
\end{aligned}
$$

- Abreviatura: Shapiro(k).

- Filtro de médias móveis de ordem $k$ :

$$
\bar{f}_{j}=\frac{1}{2 k+1} \sum_{l=j-k}^{j+k} f_{l},
$$

- Abreviatura: MA(k).

- Ajuste por mínimos quadrados local com $k$ pontos

- Ajusta localmente polinômios de ordem 2 por mínimos quadrados, considerando uma janela com $k$ pontos.

- Usou-se a função "loess" do Matlab ${ }^{\circledR}$,

- Abreviatura: loess $(\mathrm{k})$.

Apresentamos na figura 4.11 os resultados obtidos na aplicação de métodos de suavização para a solução numérica que considera a localidade do suporte das ondaletas harmônicas $(d=1)$, onde aparecem pequenas oscilações. Testamos com o método de Shapiro, que começa a a surtir efeito sobre o sinal em ordens mais altas, assim como o método "loess", que com ordens baixas praticamente não suaviza a função. O método de médias móveis reduziu bastante as oscilações, mas tem também um efeito difusivo. A escolha do tamanho da janela irá depender do tipo de oscilação gerada, com frequências maiores ou menores, e isso irá depender da escolha dos parâmetros de corte por localidade e de relação entre frequências.

Vejamos o caso de se considerar a condição inicial exponencial, por exemplo no caso onde $d=1$ e sem cortes por frequências, apresentado na figura 4.10, onde verificam-se oscilações ao longo do 

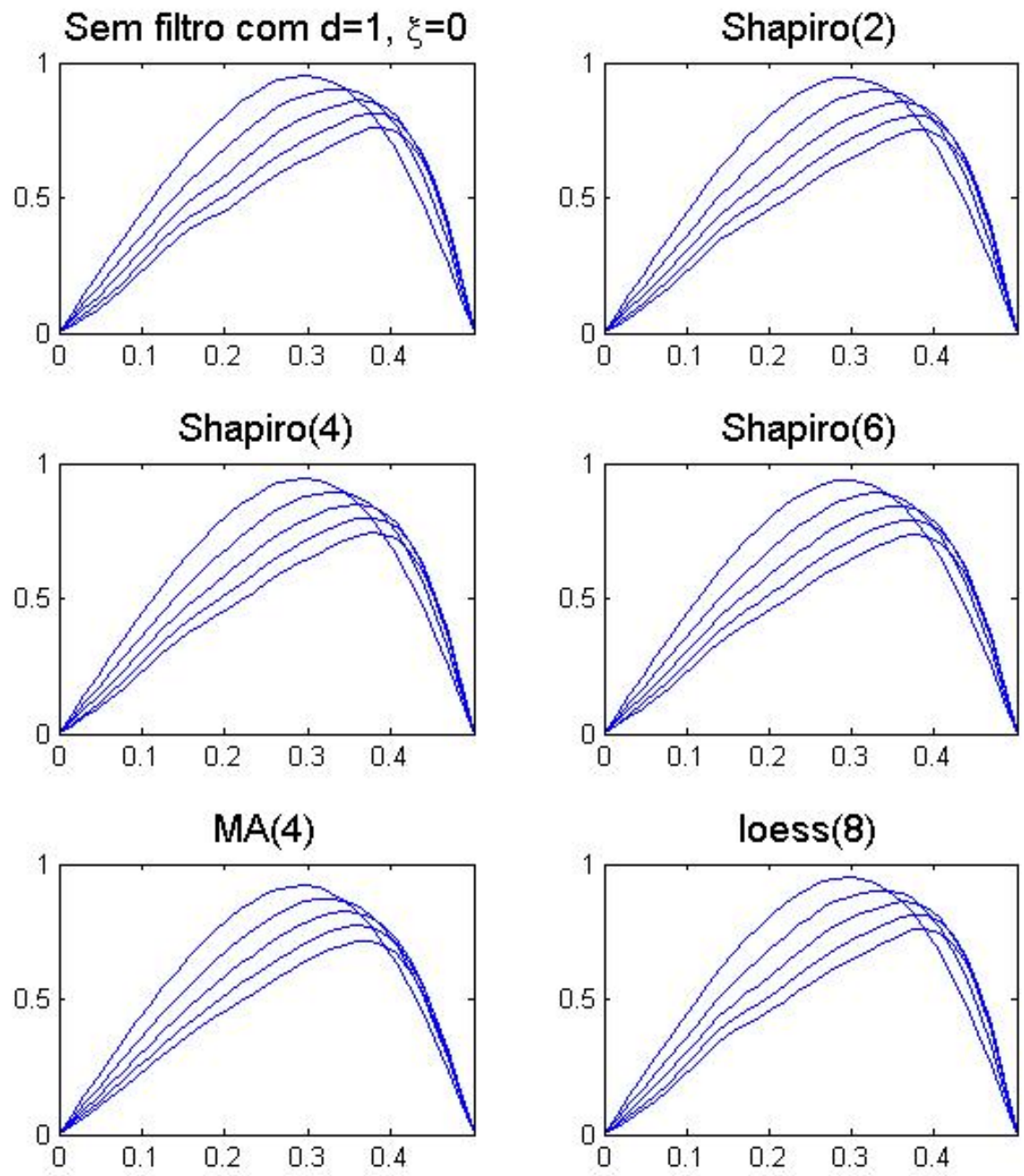

Parâmetros: $y=0.025 \mathrm{dt}=0.00097656 \quad \mathrm{~N}=64 \quad$ Niveis $\log 2(\mathrm{~N})=6$ Gráfico a cada 0.05 Cortes: Linear $(\mathrm{d})=1$ Nẫo Linear $(\mathrm{dnl})=1$ Freq. $=0$ Geral $(\mathrm{rc})=0$

Figura 4.11: No primeiro quadro (superior esquerdo) aparece a solução numérica da equação de Burgers considerando $\nu=0.025, N=64$ para a condição inicial senoidal considerando localidade do suporte da base de ondaletas harmônicas $(d=1)$ mas sem simplificações quanto às frequências. Os demais quadros mostram esta solução após ser utilizado um método de suavização. Os gráficos representam os instantes $0.05,0.1$, 0.15, 0.20 e 0.25 de tempo e apresentam somente a primeira metade de intervalo unitário.

sinal. Verifica-se na figura 4.12 que os filtros tendem a suavizar bem uma parte do sinal, mas não eliminam os ruídos em volta do sinal. Tomou-se o cuidado de, ao aumentarmos a ordem dos filtros, para eliminar melhor o ruído, não prejudicarmos características importantes da função, como a sua frente. Mas para tanto não é possível eliminar totalmente o ruído em volta do sinal. Vamos considerar outros dois casos: primeiro análogo ao anterior agora com parâmetro de corte $d=3$, na figura 4.13 , e em seguida com parâmetro de corte $d=3$ e de frequências $\xi=2$, na figura 4.14 . No caso onde $d=3$, os filtros conseguem de fato suavizar a função sem perder características de forma 
importantes da mesma. Porém no caso onde introduzimos cortes por frequências $(\xi=2)$, o tipo de oscilação gerada não é bem suavizado com o uso dos filtros.
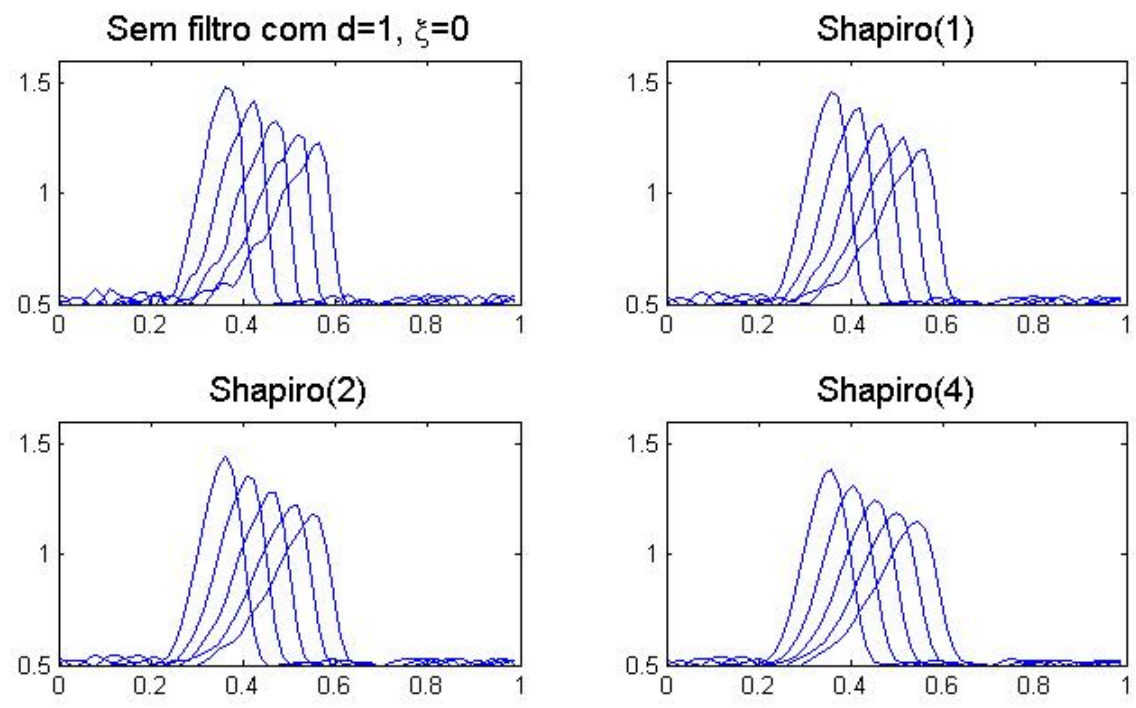

MA(2)
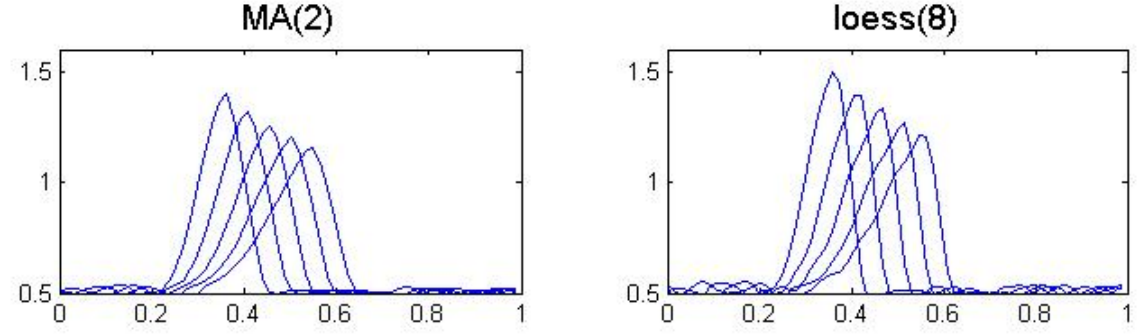

Parâmetros: $v=0.002 \mathrm{dt}=0.00097656 \quad \mathrm{~N}=64 \quad$ Niveis $\log 2(\mathrm{~N})=6 \quad$ Gráfico a cada 0.05

Cortes: Linear $(\mathrm{d})=1$ Nã̃o Linear $(\mathrm{dnl})=1$ Freq. $=0$ Geral $(\mathrm{rc})=0$

Figura 4.12: No primeiro quadro (superior esquerdo) aparece a solução numérica da equação de Burgers considerando $\nu=0.002, N=64$ para a condição inicial exponencial considerando localidade do suporte da base de ondaletas harmônicas $(d=1)$ e sem simplificações quanto às frequências $(\xi=0)$. Os demais quadros mostram esta solução após ser utilizado um método de suavização. Os gráficos representam os instantes $0.05,0.1,0.15,0.20$ e 0.25 de tempo. 

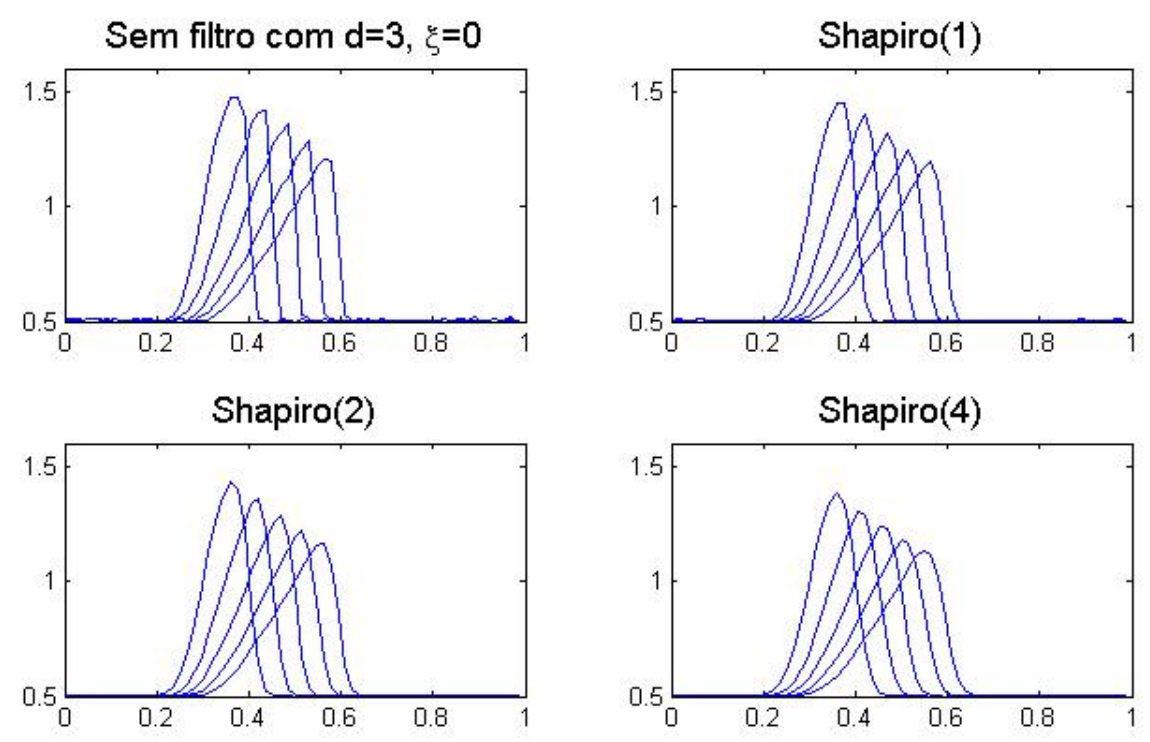

$\mathrm{MA}(2)$
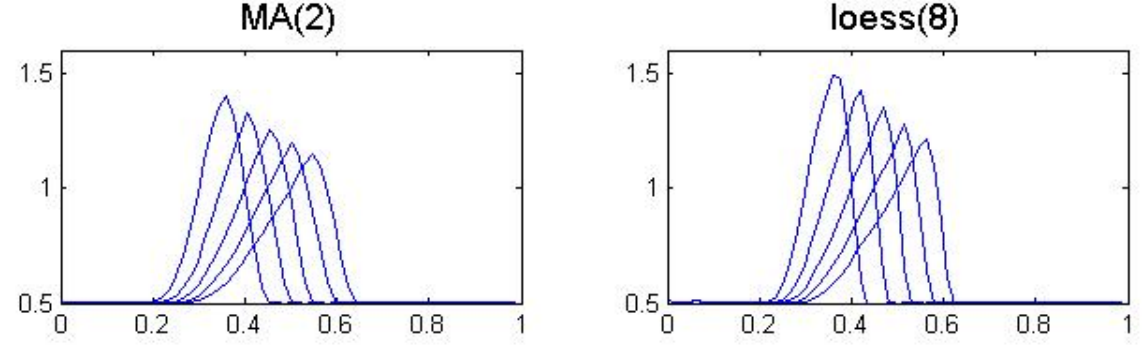

Parâmetros: $v=0.002 \mathrm{dt}=0.00097656 \mathrm{~N}=64$ Niveis $\log 2(\mathrm{~N})=6$ Gráfico a cada 0.05

Figura 4.13: No primeiro quadro (superior esquerdo) aparece a solução numérica da equação de Burgers considerando $\nu=0.002, N=64$ para a condição inicial exponencial considerando localidade do suporte da base de ondaletas harmônicas $(d=1)$ e sem simplificações quanto às frequências $(\xi=0)$. Os demais quadros mostram esta solução após ser utilizado um método de suavização. Os gráficos representam os instantes $0.05,0.1,0.15,0.20$ e 0.25 de tempo. 

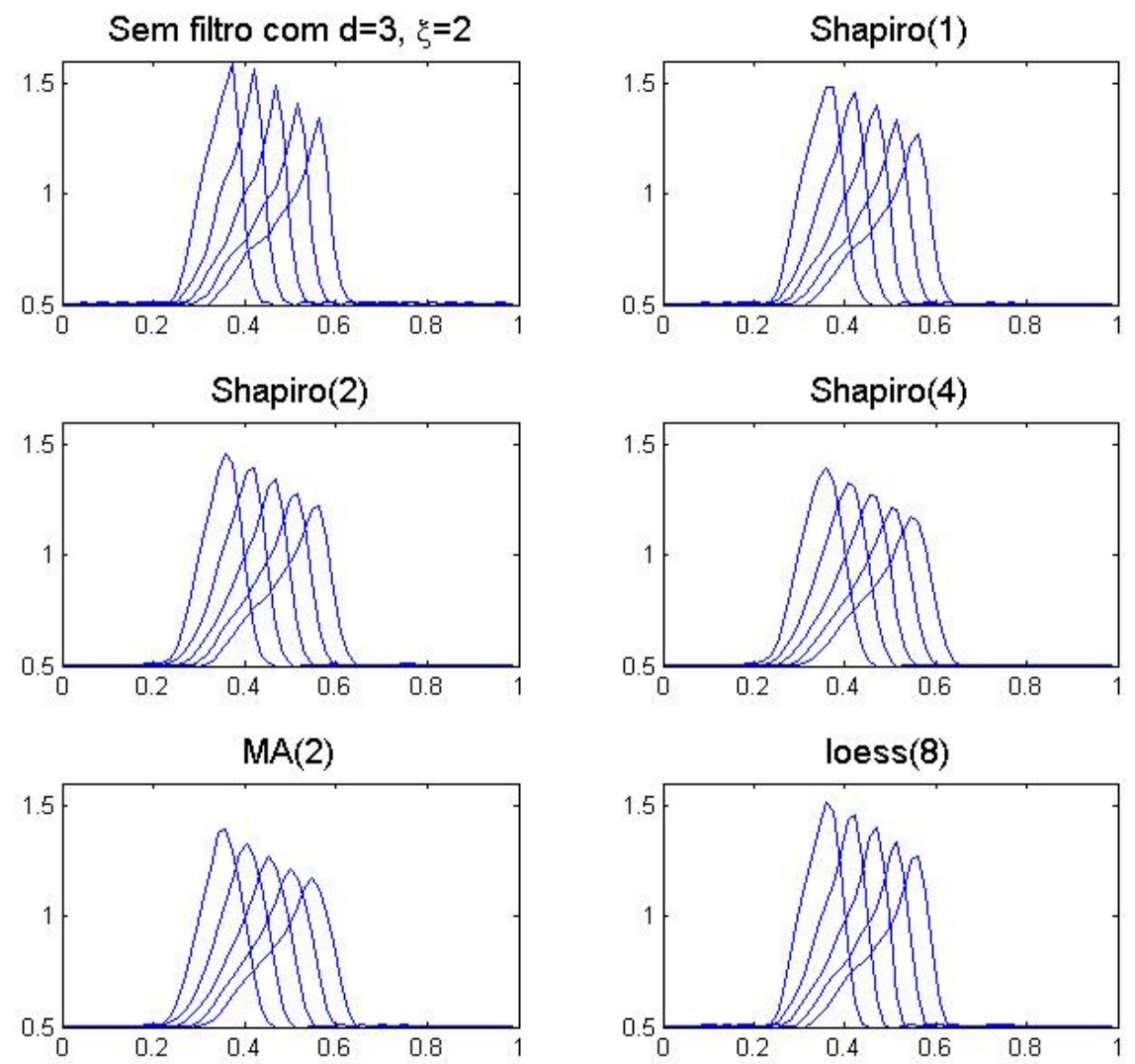

Parâmetros: $v=0.002 \mathrm{dt}=0.00097656 \mathrm{~N}=64 \quad$ Niveis $\log 2(\mathrm{~N})=6 \quad$ Gráfico a cada 0.05

Figura 4.14: No primeiro quadro (superior esquerdo) aparece a solução numérica da equação de Burgers considerando $\nu=0.002, N=64$ para a condição inicial exponencial considerando localidade do suporte da base de ondaletas harmônicas $(d=1)$ e sem simplificações quanto às frequências $(\xi=2)$. Os demais quadros mostram esta solução após ser utilizado um método de suavização. Os gráficos representam os instantes $0.05,0.1,0.15,0.20$ e 0.25 de tempo. 


\subsubsection{Localidade do sinal}

Agora vamos analisar o comportamento dos coeficientes de ondaletas harmônicas, e verificarmos se é possível obtermos informações sobre a posição no espaço da solução ao longo do tempo. Simulamos a equação de Burgers com viscosidade $\nu=0.002, N=64$ pontos de malha, sem restrições de suporte por localidade $(d=0)$, com o mesmo método Runge-Kutta de quarta ordem para a discretização temporal com $\Delta t=\Delta x / 16$. A simulação foi até o tempo de 0.6 , para o qual vamos analisar os coeficientes de ondaletas harmônicas. Na figura 4.15 temos a evolução do sinal a cada 0.1 unidades de tempo e os coeficientes de ondaletas harmônicas e de Fourier no instante de tempo final (0.6). Lembrando que um coeficiente de ondaletas harmônicas $a_{j, k}$ é centrado no valor $\frac{k}{2^{j}}$, podemos verificar algumas características do sinal. Primeiro note que o pulso está restrito à um subintervalo do $\left[0, \frac{1}{2}\right]$, e que o pico (local onde a função atinge valor máximo) ocorre entre os valores 0.3 e 0.35 na escala de espaço. Para os níveis 0 e 1 há pouca informação sobre localidade. Para o nível 2, verifica-se que o coeficiente de maior valor é de translação $k=1$, indicando a presença do sinal próximo de $1 / 4$ no espaço. No nível 3 o coeficiente mais significativo é o de translação $k=3$, indicando a presença do sinal em $3 / 8 \approx 0.375$ do intervalo unitário. Já o nível 4 , que é o de maior detalhes, indica o coeficiente de translação $k=6$ como de maior valor em módulo, e o de segundo maior valor com translação $k=5$, o que representa no intervalo unitário que o sinal tem localização entre $5 / 16 \approx 0.3125$ de $6 / 16 \approx 0.375$, que é uma boa aproximação do pico do pulso. A idéia é que, em modelos mais complexos, possam ser extraídas informações sobre a localidade do sinal a partir apenas de seus coeficientes de ondaletas. Note, nesta mesma figura, que os coeficientes de Fourier nada dizem sobre a localidade do sinal. 

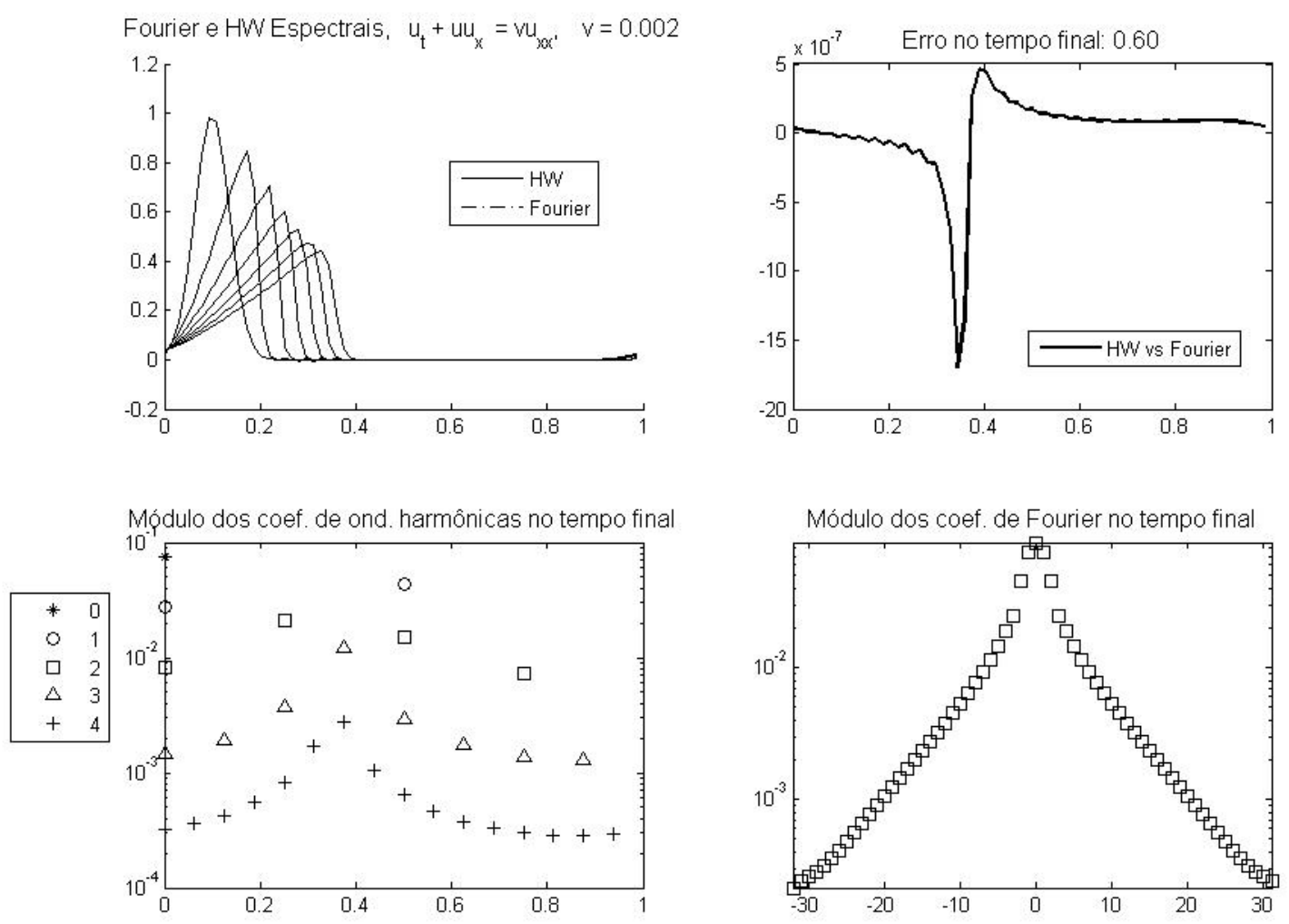

Parâmetros: $d t / d x=0.0625 d t=0.00097656 \quad N=64 \quad$ Niveis $\log 2(N)=6 \quad$ Gráfico a cada 0.1 Cortes: Linear $(\mathrm{d})=0$ Não Linear $(\mathrm{dnl})=0$ Freq. $=0$ Geral $(\mathrm{rc})=0$

Figura 4.15: Solução numérica da equação de Burgers considerando $\nu=0.002, N=64$ para a condição inicial de pulso com a base completa de ondaletas harmônicas. As curvas do gráfico do canto esquerdo superior mostram a condição inicial e as aproximações com ondaletas harmônicas (HW) e Fourier a cada 0.1 unidades de tempo. Os gráficos na parte inferior mostram os módulos dos coeficientes de ondaletas harmônicas e Fourier. 
Vejamos um outro exemplo, com condição inicial exponencial e $\nu=0.002$, apresentado na figura 4.16. Observemos o nível 4 dos coeficientes, e vejamos como os elementos mais evidentes (de maiores valores em módulo) referem-se justamente aos locais de maiores gradientes do sinal (frentes), que evoluem ao longo do tempo. Para este nível (4), no instante inicial os coeficiente de destaque são $k=4$ e $k=6$, fazendo referencia a frentes nas posições $4 / 16 \approx 0.25$ e $6 / 16 \approx 0.375$ no espaço, e de fato aproximam bem as posições de maiores gradientes da função. No instante seguinte, destaca-se o coeficiente onde $k=9$, que refere-se à posição $9 / 16 \approx 0.56$ no espaço, que novamente é uma boa aproximação do local da frente. No último instante destacam-se os coeficientes $k=11$ e $k=12$, que referem-se às posições $11 / 16 \approx 0.68$ e $12 / 16 \approx 0.75$ no espaço, coincidindo com o local da frente. Interpretações semelhantes podem ser feitas em outros níveis, lembrando que quanto maior o nível maior o nível de detalhe, referindo-se a locais da função com escalas menores (frequências maiores). Com níveis menores obtém-se informações sobre locais da função onde há oscilações de escalas maiores (baixas frequências).
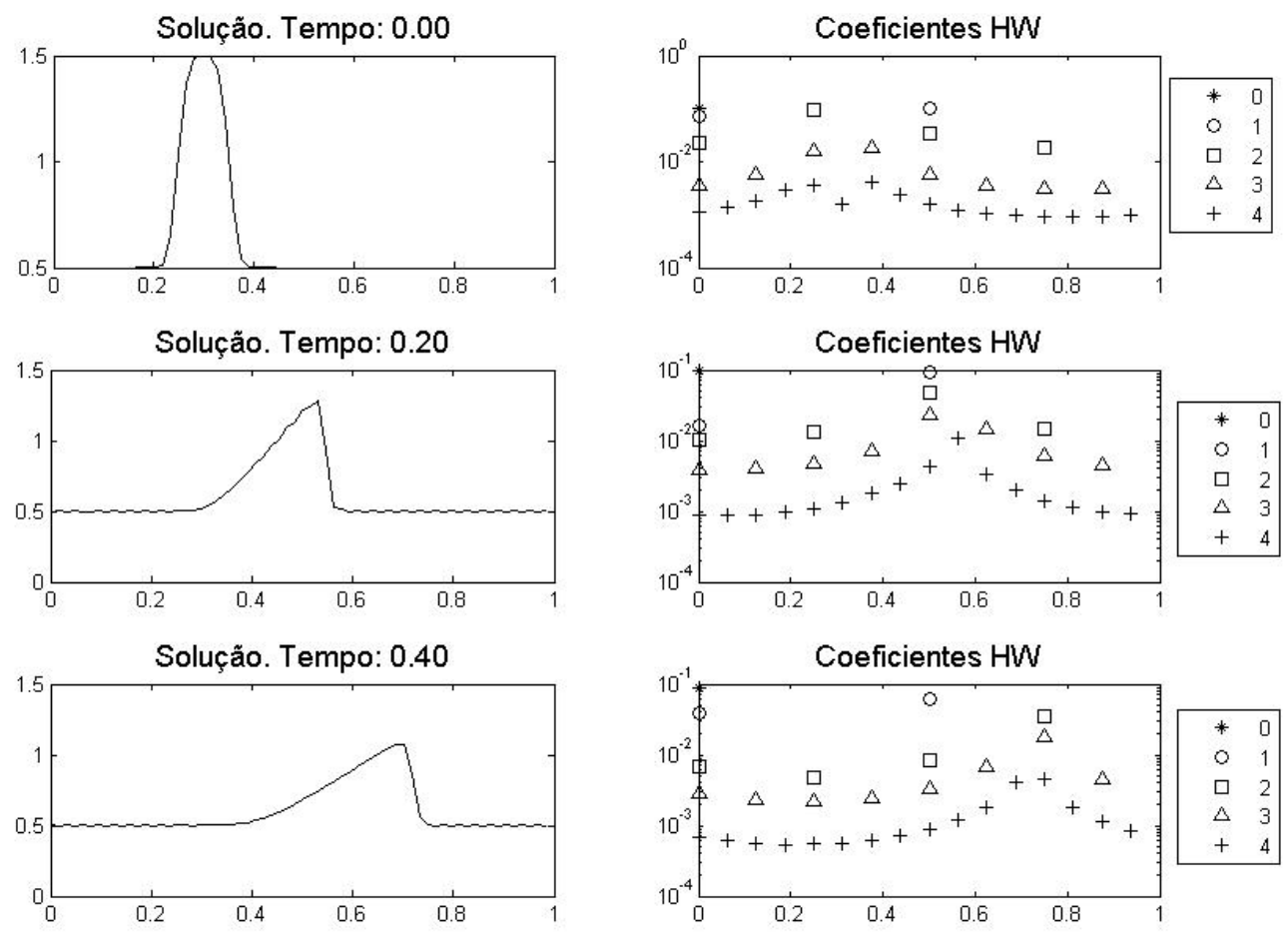

Parâmetros: $d t / d x=0.0625 \mathrm{dt}=0.00097656 \quad \mathrm{~N}=64$ Niveis $\log 2(\mathrm{~N})=6 \quad$ Cortes: Linear $(\mathrm{d})=0$ Nẫo Linear $(\mathrm{dnl})=0 \quad \mathrm{Freq} .=0 \quad$ Geral $(\mathrm{rc})=0$

Figura 4.16: Solução numérica da equação de Burgers considerando $\nu=0.002, N=64$ para a condição inicial exponencial com a base completa de ondaletas harmônicas. Na primeira coluna aparecem as soluções numéricas em 3 instantes de tempo e ao lado os respectivos coeficientes de ondaletas harmônicas (HW) em módulo e escala logarítmica. 


\subsubsection{Abordagem Pseudo-Espectral}

A maior parte do tempo de execução consumido pelo método de Galerkin com ondaletas harmônicas deve-se aos cálculos da projeção da componente não linear da equação de Burgers. O mesmo ocorre quando usamos a base de Fourier. Para melhorar a eficiência computacional é possível nos valermos de um método pseudo-espectral. Para este método calcula-se a projeção da componente não linear no domínio espacial, e não no domínio espectral. Desta forma faz-se uso da rapidez das transformadas tanto de ondaletas harmônicas quanto de Fourier. Um possível problema é a interferência de frequência altas nas baixas frequências, fenômeno conhecido por "aliasing". Para evitar este problema consideramos a malha espacial com $\frac{3 N}{2}$ pontos, e zeramos as frequências mais altas (maiores do que $N$ ).

Testamos o método pseudo-espectral com ondaletas harmônicas considerando $\nu=0.002, N=64$ e com condição inicial exponencial. Na figura 4.17 mostramos os resultados para o método, em comparação com Fourier. Nota-se que os dois métodos são essencialmente equivalentes. Como na equação de burgers temos uma componente linear, devido à difusão, podemos utilizar a propriedade de localidade para reduzir o custo dos cálculos desta parte linear. Para tanto tomamos, por exemplo, $d=3$. Mostramos os resultados na figura 4.18. Neste caso a diferença entre o método com ondaletas harmônicas e Fourier é fruto somente dos cômputos da parte linear, e tem ordem semelhante ao que foi visto na seção 4.1 .

Destacamos na tabela 4.2 os tempos de execução do método pseudo-espectral com ondaletas harmônicas, com parâmetros $d=0$ e $d=3$. Nota-se uma redução drástica do custo computacional em relação ao método de Galerkin. Porém, ainda assim, o custo computacional é ligeiramente maior do que o método pseudo-espectral com base de Fourier, que aparece na tabela 4.3. Mas que é compensado pelo fato de termos agora uma base que pode dar informações sobre localidade no domínio espectral.

O fato de termos reduzido o custo computacional nos permite analisar a evolução de aproximações da solução da equação de Burgers para valores de $N$ maiores. Vejamos o comportamento do método para a condição inicial senoidal com viscosidade 0.0025 , considerando $N=2^{10}$, com resultados na figura 4.19. Note que a imposição do parâmetro $d=3$ gera algum ruído na solução, mas a mesma tem sua forma essencialmente preservada. 

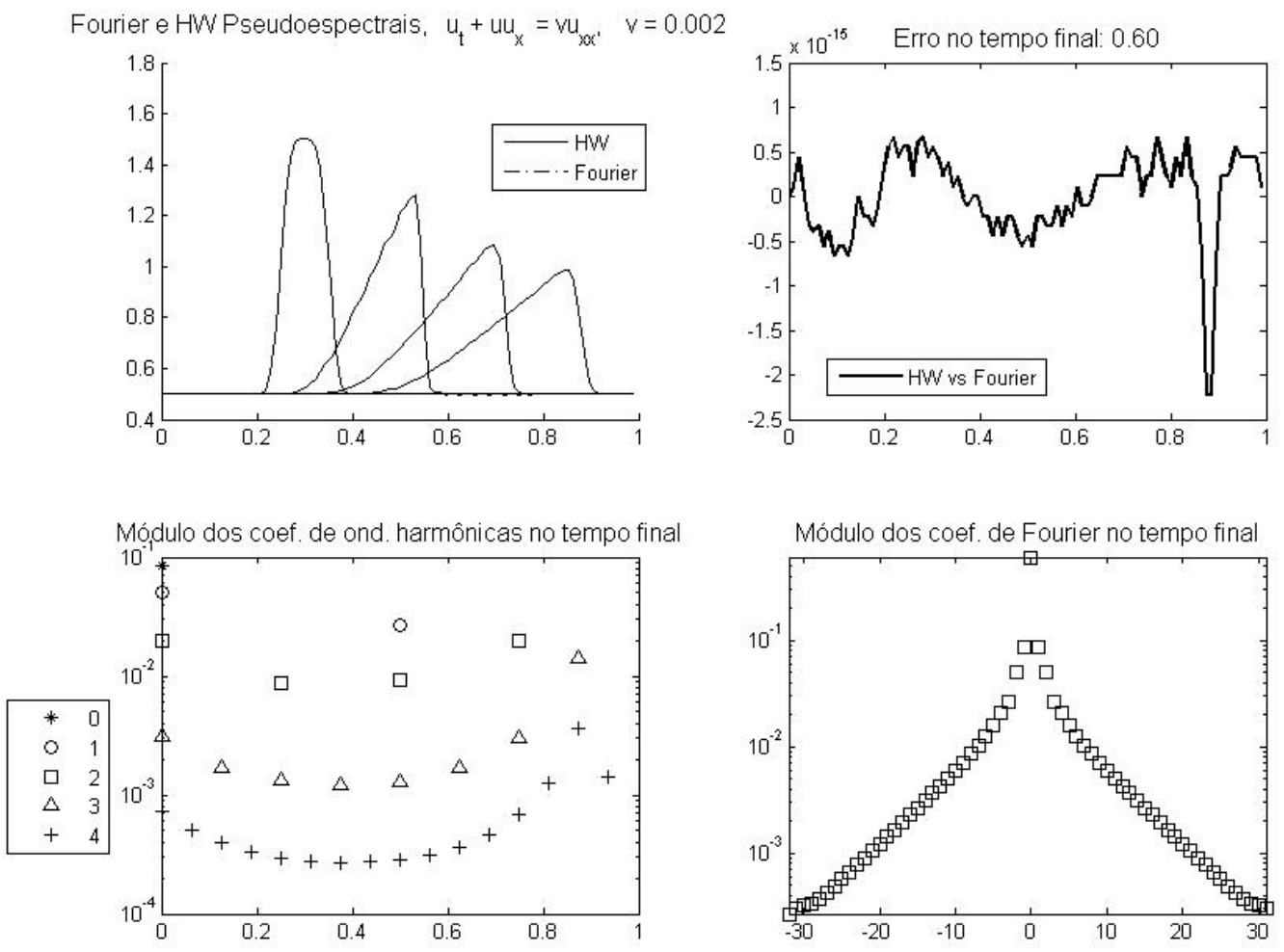

Parâmetros: $d t / d x=0.0625 \mathrm{dt}=0.00097656 \mathrm{~N}=64 \quad$ Niveis $\log 2(\mathrm{~N})=6$ Gráfico a cada 0.2

Figura 4.17: Solução numérica da equação de Burgers considerando $\nu=0.002, N=64$ para a condição inicial exponencial com a base completa de ondaletas harmônicas utilizando uma abordagem pseudo-espectral tanto para Fourier como para ondaletas harmônicas. Os gráficos na parte inferior mostram os módulos dos coeficientes de ondaletas harmônicas e Fourier. 

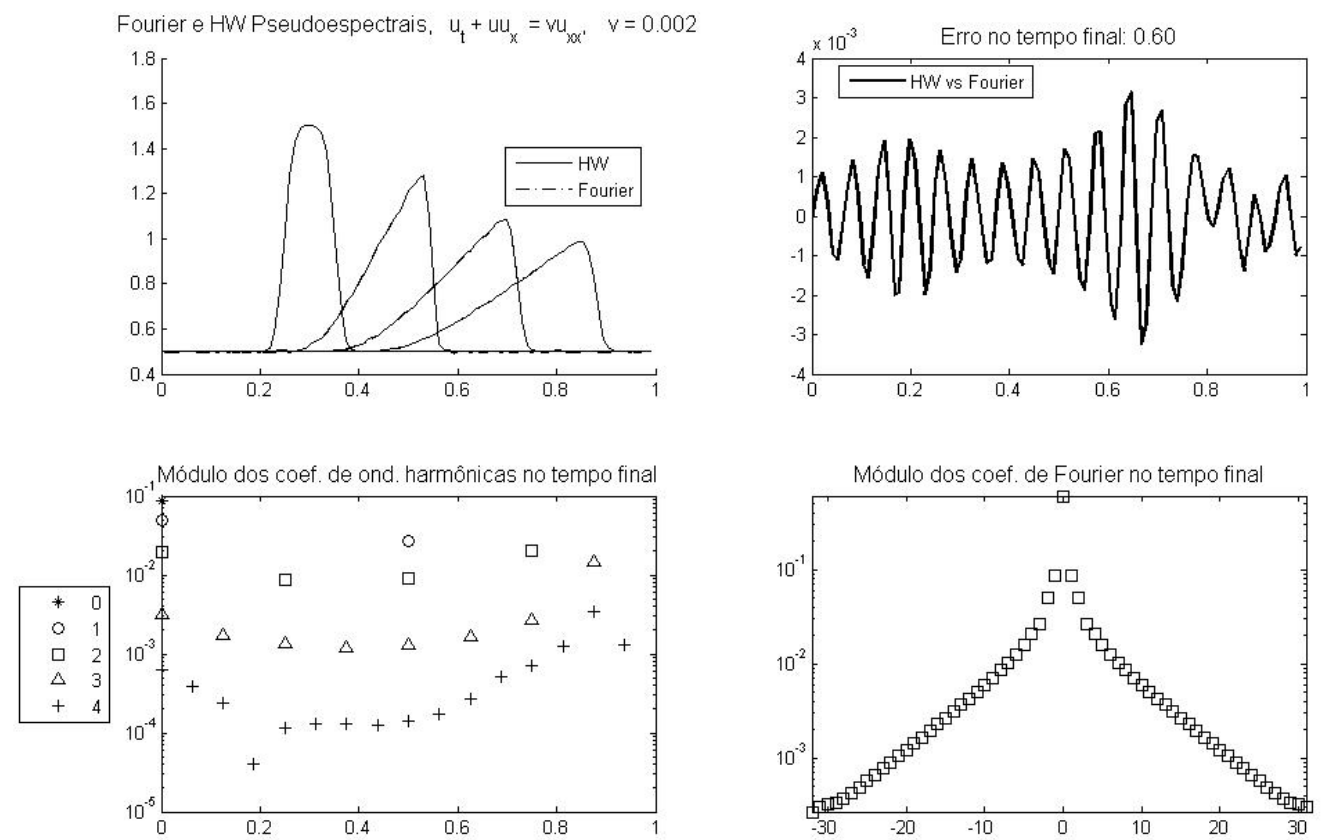

Parâmetros: $d t^{\prime} d x=0.0625 \mathrm{dt}=0.00097656 \mathrm{~N}=64$ Niveis $\log 2(\mathrm{~N})=6$ Gráfico a cada 0.2 Cortes: Linear $(\mathrm{d})=3$

Figura 4.18: Solução numérica da equação de Burgers considerando $\nu=0.002, N=64$ para a condição inicial exponencial com a base completa de ondaletas harmônicas utilizando uma abordagem pseudo-espectral tanto para Fourier como para ondaletas harmônicas. Utilizamos um corte por localidade de ondaletas harmônicas $(d=3)$ na parte linear da equação (difusão). Os gráficos na parte inferior mostram os módulos dos coeficientes de ondaletas harmônicas e Fourier. 


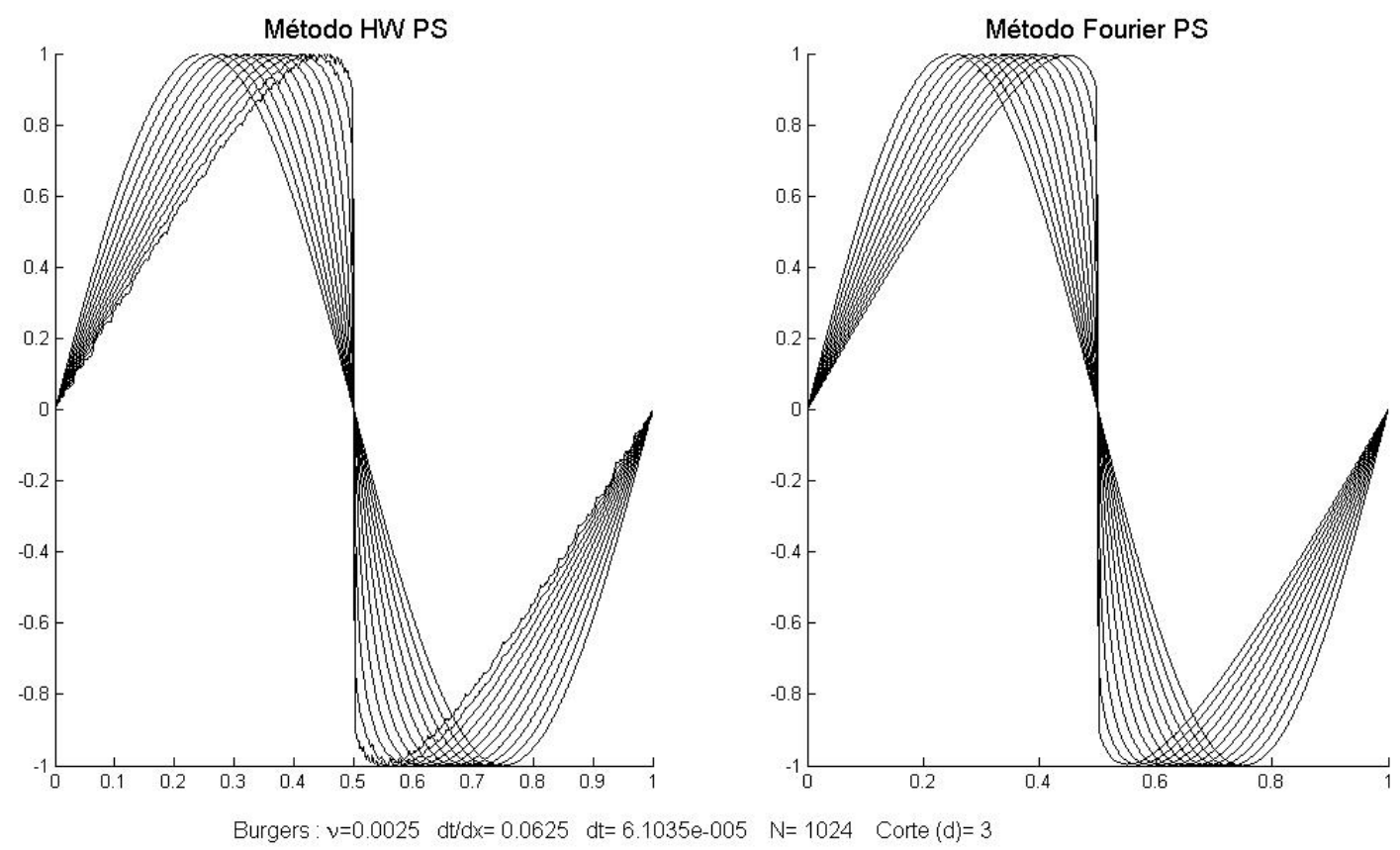

Figura 4.19: Solução numérica da equação de Burgers considerando $\nu=0.0025, N=1024$ para a condição inicial senoidal com a base completa de ondaletas harmônicas utilizando uma abordagem pseudo-espectral (PS) tanto para Fourier como para ondaletas harmônicas (HW). Utilizamos um corte por localidade de ondaletas harmônicas $(d=3)$ na parte linear da equação (difusão). As imagens aparecem a cada 0.02 instantes de tempo.

Consideramos agora a condição inicial senoidal truncada, isto é,

$$
f(x)=\left\{\begin{array}{l}
\sin (2 \pi x), \quad 0 \leq x \leq 1 / 2, \\
0, \quad c c
\end{array}\right.
$$

Este exemplo é usado no trabalho de Schult e Wyld (1992), com viscosidade $\nu=5.10^{-4}$, utilizando um método de Galerkin com ondaletas de Daubechies e refinamento local. Reproduzimos um dos resultados de Schult e Wyld (1992) na figura 4.20. No experimento foi utilizado um método semiimplícito de segunda ordem para a discretização temporal. Em seguida na figura 4.21 mostramos os resultados obtidos com ondaletas harmônicas, com método pseudo-espectral. Os resultados obtidos com ondaletas harmônicas são muito semelhantes aos obtidos com ondaletas de Daubechies. Destacamos no lado direito da mesma figura 4.21 os coeficientes de ondaletas harmônicas para o instante de tempo 0.8. Percebe-se que em todos os níveis o coeficiente de maior valor em módulo é aquele que refere-se à posição da frente de propagação. 


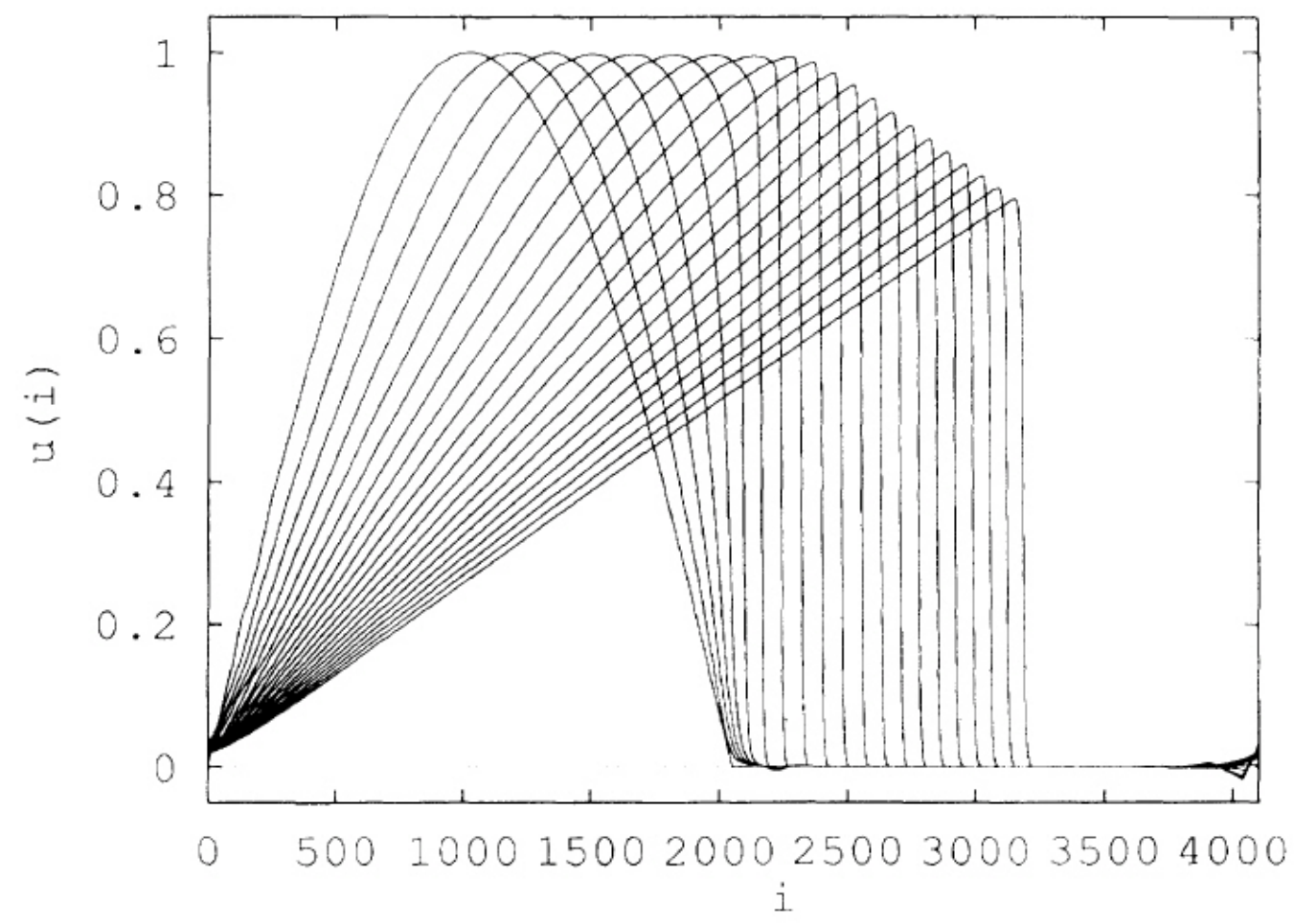

Figura 4.20: Solução numérica da equação de Burgers considerando $\nu=5 \cdot 10^{-5}, N=2^{12}$ para a condição inicial senoidal truncada com um método de Galerkin utilizando ondaletas de Daubechies e refinamento local na região da frente de onda. Os gráficos aparecem a cada 160/4096 unidade de tempo. 

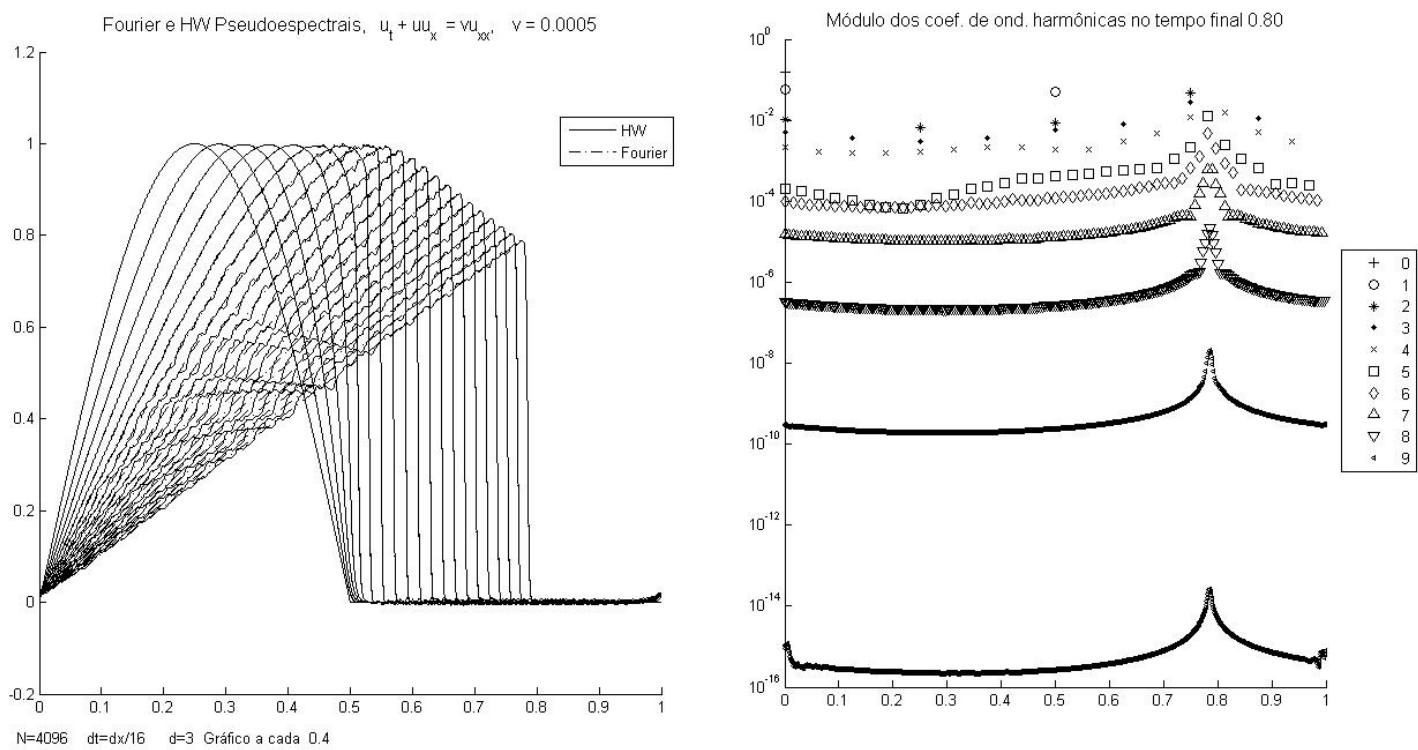

Figura 4.21: Solução numérica da equação de Burgers considerando $\nu=5 \cdot 10^{-5}, N=2^{12}$ para a condição inicial senoidal truncada com métodos pseudo-espectrais de Fourier e de ondaletas harmônicas. Os gráficos aparecem a cada 0.4 unidade de tempo. Foi utilizado corte por localidade $d=3$. 


\section{Capítulo 5}

\section{Aplicações em Meteorologia}

Neste capítulo vamos avaliar o desempenho do método de Galerkin, com base de ondaletas harmônicas, em um modelo meteorológico relacionado à propagação de frentes de precipitação em regiões tropicais. Trataremos do caso unidimensional de um modelo proposto inicialmente por Frierson, Majda e Pauluis (2004), conhecido por modelo de clima tropical, e que pode ser visto como um modelo de propagação de frentes de precipitação.

\subsection{Modelo de propagação de frentes de precipitação}

O modelo de propagação de frentes de precipitação unidimensional proposto em Frierson, Majda e Pauluis (2004) é um caso particular de um modelo de clima tropical proposto no mesmo artigo. Começamos por detalhar brevemente a construção do modelo de clima tropical em questão. Destacamos a seguir, de forma esquemática, as etapas de sua construção.

1. Equações de Navier-Stokes

$\downarrow$ Considera-se a densidade constante, exceto no acoplamento com a gravidade, onde assume-se que a densidade varia linearmente com a temperatura. Esta hipótese está fundamentada no fato de a atmosfera concentrar variações de densidade apenas na sua camada mais inferior ( $10 \%$ da altura total da atmosfera), e nos leva a olhar o problema como incompressível. É adequado para estudos sobre convecção natural.

$\downarrow \quad$ Toma-se uma aproximação hidrostática, onde desconsideram-se acelerações verticais.

$\downarrow$ Domínio restrito ao plano $\beta$ equatorial, no qual consideram-se apenas regiões tropicais, isto é, de baixas latitudes. No plano $\beta$ aproximam-se os valores de $\cos (\phi)$ e $\sin (\phi)$ respectivamente por 1 e 0 , onde $\phi$ indica latitudes.

2. Equações de Boussinesq hidrostáticas no plano $\beta$ equatorial 1

$\downarrow$ Decomposição vertical usando expansão dos campos em polinômios trigonométricos, $P_{j}(z)=\cos (j z)$, para $j=0,1, \ldots$, e depois um procedimento conhecido por truncamento de Galerkin.

\footnotetext{
${ }^{1}$ Detalhes sobre a construção das equações de Boussinesq podem ser vistos em Holton (2004).
} 
$\downarrow \quad$ No truncamento de Galerkin mantém-se apenas os coeficientes referentes aos elementos de base $P_{0}(z)=1$ (Modo barotrópico) e $P_{1}(z)=\cos (z)$ (Primeiro modo baroclínico).

$\hookrightarrow$ Modo barotrópico - Densidade depende apenas da pressão e vice versa

$\hookrightarrow$ Primeiro modo baroclínico - Densidade depende de pressão e temperatura. Ventos na camada inferior da troposfera tem mesma magnitude mas sinais opostos aos ventos da camada superior da troposfera. Justifica-se em áreas de grandes convecções.

$\downarrow \quad$ Inclui-se a equação de dinâmica de umidade simplificada

$\downarrow \quad$ Parametrizações físicas - forçantes do modelo

$\hookrightarrow$ Radiação Térmica

$\hookrightarrow$ Fluxo de calor sensível

$\hookrightarrow$ Arrasto por fricção

$\hookrightarrow$ Evaporação

$\hookrightarrow$ Precipitação

3. Modelo de Clima Tropical

$\hookrightarrow$ Equações para os campos:

- Velocidades do modo barotrópico $(\bar{U})$

- Velocidades do primeiro modo baroclínico $\left(U\right.$ ou $\left.U_{1}\right)$

- Perturbação da Temperatura $(T)$

- Umidade $(q)$

$\hookrightarrow$ A precipitação é calculada a partir da umidade e temperatura

Algumas considerações importantes são feitas a respeito da inclusão da equação da umidade e das parametrizações físicas, que destacamos a seguir. As parametrizações físicas são geralmente incorporadas no modelo através de forçantes com relaxações, isto é, amortecimentos, ou incrementos, exponenciais de uma das quantidades $U, T$ ou $q$ com taxas definidas por tempos de relaxação empíricos. O tempo de relaxação é em geral calculado a partir do tempo do qual um sistema demora para voltar a uma situação de equilíbrio depois de ser perturbado.

1. Arrasto por fricção:

$\hookrightarrow$ Grande na superfície e pequeno em grandes altitudes.

$\hookrightarrow$ Parâmetro de relaxação $\bar{d} \approx(3.8 a 10 \text { dias })^{-1}$.

$\hookrightarrow$ Forçante $S_{U}=-\bar{d} U$.

$\hookrightarrow$ No caso sem vento barotrópico a forçante é nula.

2. Radiação Térmica: 
$\hookrightarrow$ Resfriamento Newtoniano (no qual a taxa de perda de calor de um corpo é proporcional a diferença de temperatura do corpo em relação ao ambiente).

$\hookrightarrow$ Relaxa temperatura a um perfil de equilíbrio de radiação em um certo tempo.

$\hookrightarrow$ Parâmetro de relaxação $d_{T} \approx(20 \text { dias })^{-1}$,

$\hookrightarrow$ Forçante $S_{T, R}=-d_{T}\left(T-T_{e q}\right)$.

$\hookrightarrow T_{e q}<0$ é a temperatura de equilíbrio.

$\hookrightarrow$ No caso sem vento barotrópico a forçante é nula.

3. Fluxo de calor sensível:

$\hookrightarrow$ Parâmetro de relaxação $d_{S H} \approx(\text { (10dias })^{-1}$,

$\hookrightarrow$ Forçante $S_{T, S H}=-d_{S H}\left(T_{s}-T\right)$.

$\hookrightarrow T_{s}$ é a temperatura na superfície, em geral dada pela temperatura ao nível do mar.

$\hookrightarrow$ No caso sem vento barotrópico a forçante é nula.

$\hookrightarrow$ É uma forçante de menor impacto que as demais, e será desprezada.

4. Evaporação:

$\hookrightarrow$ Parâmetro de relaxação $d_{q} \approx(10 \text { dias })^{-1}$,

$\hookrightarrow$ Forçante $E=d_{q}\left(q_{s}\left(T_{s}\right)-q\right)$.

$\hookrightarrow T_{s}$ é a temperatura na superfície.

$\hookrightarrow q$ é umidade e $q_{s}$ é umidade na superfície.

$\hookrightarrow$ No caso sem vento barotrópico a forçante é nula.

5. Umidade:

$\hookrightarrow$ Microfísica de nuvens

- Vapor d'água $\left(q_{v}\right)$

- Água em nuvens $\left(q_{c}\right)$

- Água em chuva $\left(q_{r}\right)$

- Água total líquida $\left(q_{l}=q_{c}+q_{r}\right)$

$\hookrightarrow$ Considera-se $\left\langle\frac{D q_{l}}{D t}, 1\right\rangle=0$

$\hookrightarrow$ Define-se a umidade como $q=\left\langle q_{v}, 1\right\rangle$

$\hookrightarrow$ Define-se um parâmetro de umidade média (Q), que é uma medição experimental da média do gradiente de umidade, também conhecido por estratificação de umidade bruta, $0<Q<1$.

$\hookrightarrow$ Princípios de conservação da umidade levam à equação para a mesma, dependente da precipitação e evaporação. 
6. Precipitação:

$\hookrightarrow P=\left.v_{t} q_{r}\right|_{z=0}$, onde $v_{t}$ é a velocidade final dos "pingos" de água e $q_{r}$ é a variável que indica a parcela de chuva do ar.

$\hookrightarrow P=\frac{1}{\tau}(q-\tilde{q}(T))^{+}$, onde $(a)^{+}=\{a$, se $a>0 ; 0$ caso contrário $\}$,

$\hookrightarrow \tilde{q}(T)$ é um padrão de referência de umidade limite para o qual pode se ter chuva. Comumente dado pela expressão $\tilde{q}(T)=\hat{q}+\alpha T$, onde $\hat{q}$ indica uma umidade limite média e $\alpha$ correlaciona o limite de umidade com as variações de temperatura. Mostra-se que $\alpha>-Q$.

$\hookrightarrow \tau$ é o tempo de ajuste convectivo, que é um parâmetro de relaxação com valores usualmente entre 2 e 24 horas.

Com isso já possível escrevermos as equações do modelo de clima tropical para o caso unidimensional, para o qual o vento barotrópico pode ser visto como constante $\bar{u}$ e há somente um modo de vento baroclínico $u$. Pode se entender a constante de vento barotrópico $\bar{u}$ como uma média de ventos, e o vento baroclínico como as variações sobre esta média.

$$
\begin{aligned}
\frac{\partial u}{\partial t}+\bar{u} \frac{\partial u}{\partial x} & =\frac{\partial T}{\partial x}+S_{U} \\
\frac{\partial T}{\partial t}+\bar{u} \frac{\partial T}{\partial x} & =\frac{\partial u}{\partial x}+S_{T, R}+S_{T, S H}+P \\
\frac{\partial q}{\partial t}+\bar{u} \frac{\partial q}{\partial x} & =-Q \frac{\partial u}{\partial x}+E-P .
\end{aligned}
$$

Além disso é possível obtermos a velocidade vertical tomando-a como $w=-\frac{\partial u}{\partial x}$. Este pode ser visto como um modelo de propagação de frentes de precipitação com vento barotrópico. Ao desconsiderarmos o vento barotrópico temos um modelo simplificado, dado pelas equações,

$$
\begin{aligned}
\frac{\partial u}{\partial t} & =\frac{\partial T}{\partial x} \\
\frac{\partial T}{\partial t} & =\frac{\partial u}{\partial x}+P \\
\frac{\partial q}{\partial t} & =-Q \frac{\partial u}{\partial x}-P
\end{aligned}
$$

Estudos mostram que o tempo de ajuste convectivo, $\tau$, denominado por parâmetro de relaxação no tempo, deve estar entre 2 e 24 horas, o que é muito pouco quando comparado com as escalas de tempo de fenômenos meteorológicos de grande escala (PAULUIS; FRIERSON; MAJDA, 2008). No limite em que $\tau \rightarrow 0$ o ajuste convectivo é instantâneo, que é caso que foi estudado por Frierson, Majda e Pauluis (2004) para a fronteira entre regiões com precipitação e sem precipitação, onde há instabilidade convectiva. Para $\tau>0$ pequeno temos uma aproximação do ajuste convectivo não instantâneo, este caso foi estudado mais a fundo em Stechmann e Majda (2006) também com enfoque na região de fronteira de precipitação. 
O modelo de propagação de frentes de precipitação unidimensional, acima descrito, com ou sem vento barotrópico, é basicamente um modelo para precipitação com base na evolução do vento zonal (sentido leste-oeste), no potencial de temperatura e na umidade. Este modelo desconsidera a força de Coriolis (força resultante do movimento de rotação da Terra), com isso pode ser visto como um modelo de circulação zonal ao redor do equador. Apesar de se tratar de um modelo unidimensional linear, e de ter diversas simplificações em relação ao modelo completo, constitui uma boa aproximação para a dinâmica dos trópicos, que possuem ventos predominantemente zonais. O modelo é adequado para, por exemplo, fenômenos como a circulação de Walker (descreve um movimento circular fechado nas direções zonal e vertical do ar devido à gradientes de temperatura), a propagação de "superclusters" (deslocamentos de grandes formações de nuvens) e a oscilação Madden-Julian (deslocamento para leste de uma célula zonal de grande escala que causa variações na convecção tropical e consequentemente influencia no regime de chuvas). Além disso, de acordo com o trabalho de Frierson, Majda e Pauluis (2004), a extensão do modelo para casos mais gerais é simples, tornando-se portanto um modelo de grande interesse de estudo.

Vamos olhar para algumas propriedades do modelo sem modo barotrópico, e possíveis conclusões sobre soluções, considerando o caso limite onde $\tau \rightarrow 0$, proposto por Frierson, Majda e Pauluis (2004). Se tivermos duas regiões adjacentes, uma seca $(P=0)$, e a outra molhada $(P>0)$, então haverá uma descontinuidade no campo de precipitação. Se definirmos como região molhada o intervalo tal que $x>0$, e a seca onde $x<0$, então a descontinuidade ocorre em $x=0$. Com esse domínio, utilizando-se das equações 5.5 a 5.6 é possível obtermos a precipitação em função da velocidade vertical $(w)$,

$$
P_{(x>0)}=\frac{(\alpha+Q)}{(1+\alpha)} w_{(x>0)}
$$

Com isso nota-se que uma descontinuidade em $P$ é gerada por uma descontinuidade em $w$, que por sua vez é gerada por uma "quina" em $u$. Portanto consideramos os campos de velocidade, temperatura e umidade contínuos, mas possivelmente descontínuos em suas derivadas, o que pode levar à formação de frentes de precipitação. Além disso fica claro que regiões de precipitação positiva são regiões de convecção, isto é, velocidade vertical positiva (ventos ascendentes).

De Frierson, Majda e Pauluis (2004) temos que a velocidade de propagação de uma onda de gravidade em uma região seca é $c_{d}= \pm 1$ (equivalente a $50 \mathrm{~m} / \mathrm{s}$ ), e a velocidade dentro de regiões molhadas $c_{m}= \pm \sqrt{\frac{1-Q}{1+\alpha}}$. Com isso temos apenas 3 tipos de frentes de precipitação, na região de fronteira entre região seca e molhada, dependo de sua velocidade e direção. Digamos que a velocidade de propagação da frente de precipitação seja dada por $s$, então temos as possibilidades que seguem,

- Frentes secantes, ou desumidificantes, na direção de seco para molhado, com velocidade de propagação

$$
\sqrt{\frac{1-Q}{1+\alpha}}<s<1
$$

(drying front). 
- Frentes umidificantes lentas, na direção de molhado para seco, com velocidade de propagação

$$
0<s<\sqrt{\frac{1-Q}{1+\alpha}}
$$

(slow moistening front)

- Frentes umidificantes rápidas, na direção de molhado para seco, com velocidade de propagação

$$
s>1
$$

. (fast moistening front).

Se 0 intervalo de interesse possuir diversas regiões com diferentes características de umidade, e consequentemente precipitação, então podemos ter combinações das 3 frentes descritas acima. Um caso interessante é o das frentes atenuante (dying front), ou sumidouras, que é composto por 3 regiões: a mais a esquerda é seca, a do meio é molhada, e na interface temos uma frente desumidificante; a mais a direita é seca, e na interface com a região molhada há uma frente umidificante lenta. Neste caso, devido as diferenças de velocidade de propagação das frentes, a região molhada irá gradativamente sumir. Mostramos na figura 5.1 um diagrama ilustrando a propagação deste tipo de frente.

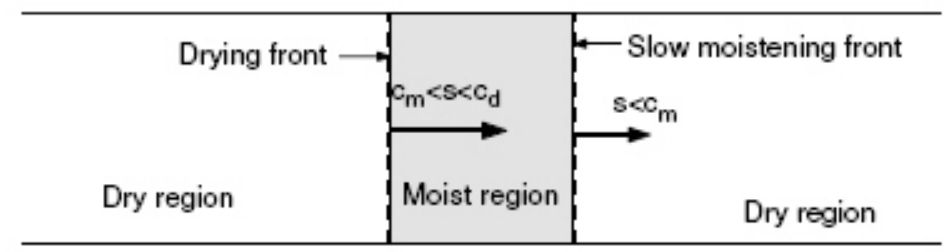

Figura 5.1: Diagrama para frente atenuante. O diagrama está em inglês, obtido em Khouider e Majda (2005). Entende-se por "dry region" e "moist region" respectivamente as regiões secas e úmidas. As setas indicam as direções e magnitudes das frentes seca e úmida lenta, respectivamente da esquerda para direita.

\subsection{Experimentos numéricos}

Vamos utilizar o método de Galerkin com ondaletas harmônicas para resolver o modelo de frentes de precipitação unidimensional sem vento barotrópico. A discretização das equações será análoga ao realizado nas equações 4.11. Porém agora expandimos e projetamos os 4 campos, $u, T, q$ e $P$, na base de ondaletas harmônicas. Novamente utilizamos um método de Runge-Kutta de quarta ordem para a resolução da parte temporal. O método de discretização espacial é mais adequado para problemas com condição periódica de fronteira, portanto começamos os experimentos com o seguinte conjunto de parâmetros e condições iniciais (ilustrados na figura 5.2). 


$$
\begin{aligned}
\alpha & =0.6 \\
Q & =0.9 \\
\hat{q} & =0.9 \\
\tau & =0.00625 \\
u & = \begin{cases}0.01 x & 0 \leq x<\frac{1}{2} \\
-0.01 x+0.01 & \frac{1}{2} \leq x<1\end{cases} \\
T & = \begin{cases}-0.0073 x+0.0073 / 2 & 0 \leq x<\frac{1}{2} \\
+0.0073 x-0.0073 / 2 & \frac{1}{2} \leq x<1\end{cases} \\
q & =\hat{q}+\alpha T
\end{aligned}
$$
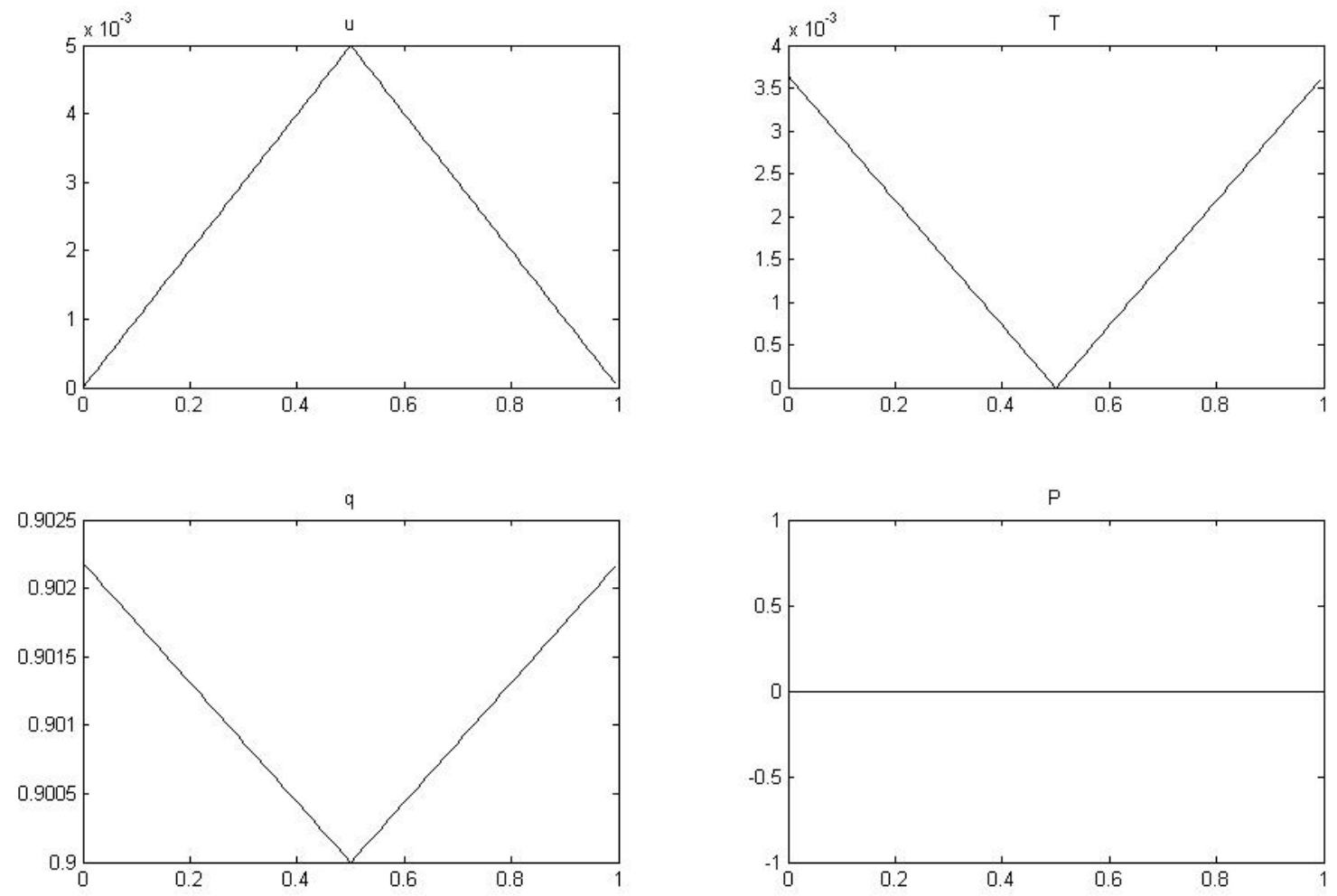

Parâmetros: Tempo=0 N=128 qhat $=0.9 \quad Q=0.9 \quad \alpha=0.6 \quad \tau=0.00625 \quad$ Dying front sem descont.

Figura 5.2: Condições iniciais para um caso de frente atenuante com fronteiras periódicas utilizando 128 pontos de grade.

Estes parâmetros e condições iniciais foram obtidos seguindo o método proposto em Khouider e Majda (2005) para construção de frentes atenuantes, e portanto esperamos que de fato ocorra este 
tipo de frente. O parâmetro de relaxação $\tau$ é equivalente a a 30 minutos em escala dimensional. Utilizando o método de Galerkin com ondaletas harmônicas obtivemos os seguintes resultados. Para facilitar a visualização gráfica transladamos em 0.5 os campos no eixo $x$. Começaremos analisando o caso sem cortes por localidade.

Na figura 5.3 apresentamos o instante de tempo $t=0.15$, onde nota-se uma região com precipitação (à esquerda) e outra sem precipitação (à direita). Além dos valores dos campos de interesse, apresentamos na mesma figura os coeficientes de ondaletas harmônicas paras os respectivo campos. É interessante notarmos que nos níveis mais altos os coeficientes de destaque são justamente aqueles referentes ao locais de descontinuidade, ou gradiente elevado. Apresentamos nas figuras subsequentes 5.3 até 5.7 a evolução dos campos ao longo do tempo, para alguns instantes. É possível percebermos que a região seca esta sendo lentamente invadida por umidade nas proximidades da metade do intervalo, assim como a frente seca esta invadindo a região úmida no começo do intervalo de forma mais rápida. Com o passar do tempo a região com precipitação vai se tornando menor, até que no último instante de tempo não há mais chuva no intervalo. É possível percebermos que os coeficientes de ondaletas harmônicas são mais significativos nas regiões onde há precipitação. Na figura 5.8 apresentamos os gráficos dos campos em diagramas $t \times x$, com o valor do campo dado pela intensidade da cor seguindo a escala ao lado de cada gráfico. Com essa figura é possível estimarmos as velocidades de propagação das frentes. Para a frente úmida lenta temos uma velocidade de aproximadamente $\frac{0.58-0.5}{0.85} \approx 0.09$, e para frente seca temos $\frac{0.58-0}{0.85} \approx 0.68$, ambas com direção da esquerda para direita. Em seguida destacamos na figura 5.9 a evolução do campo de precipitação e de seus coeficientes de ondaletas harmônicas. Nesta figura queremos ressaltar a característica de localidade da base de ondaletas harmônicas, onde fica claro que os coeficientes marcam a posição da região de precipitação e da frente de precipitação. Note, por exemplo, que nos primeiros instantes, onde a região de precipitação é relativamente larga, os coeficientes de maiores valores são os que se referem a cada uma das frentes presentes. 

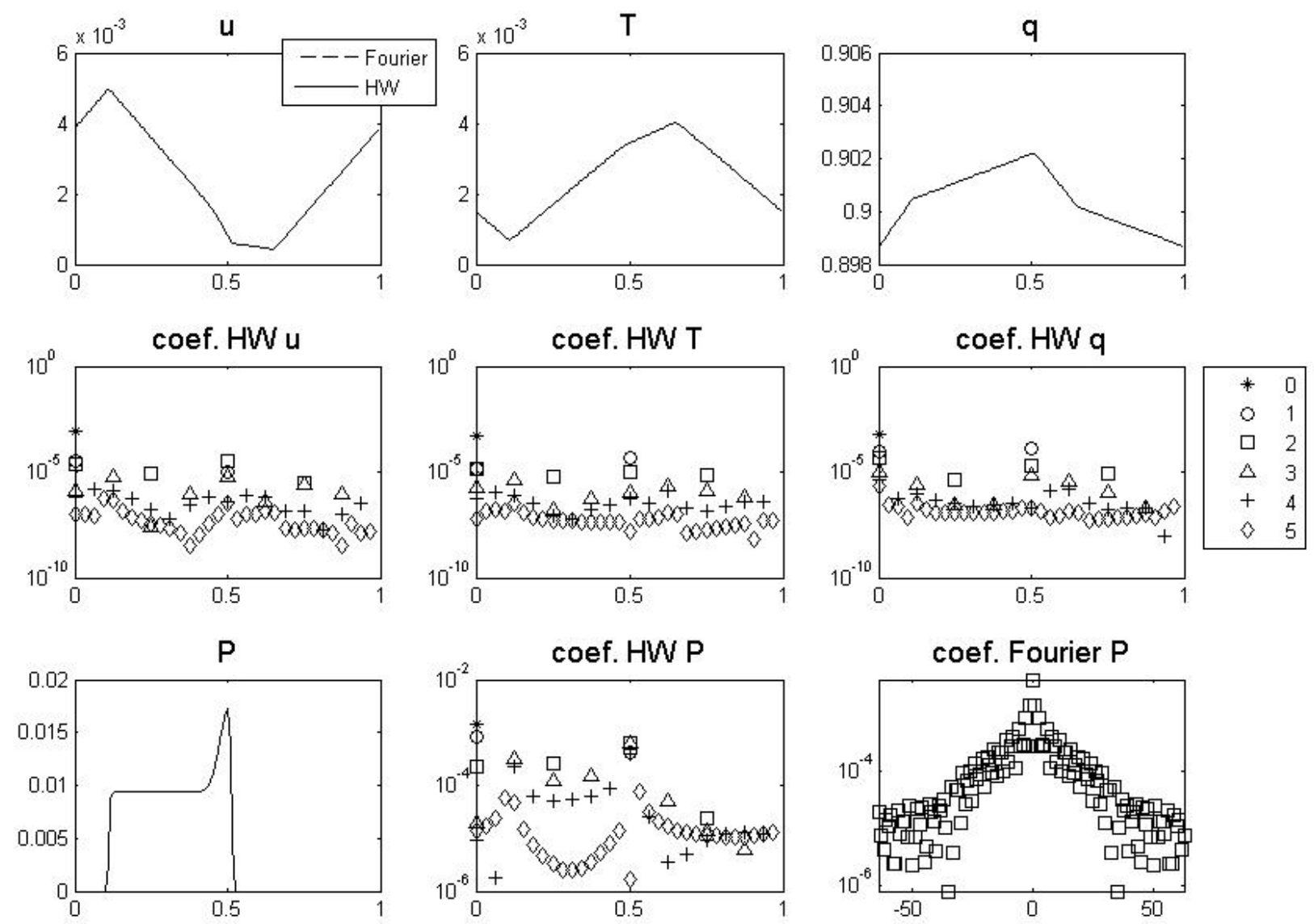

Parâmetros: Tempo=0.15 N=128 qhat =0.9 Q =0.9 $\alpha=0.6 \quad \tau=0.00625$ Dying front sem descont.

Figura 5.3: Frente atenuante no tempo 0.15 para as condições iniciais dadas nas equações 5.11. Os gráficos indicados por "coef." mostram os coeficientes de ondaletas harmônicas (HW) em módulo divididos por níveis. Os campos foram transladados em 0.5 no eixo $x$ para facilitar visualização das frentes. 

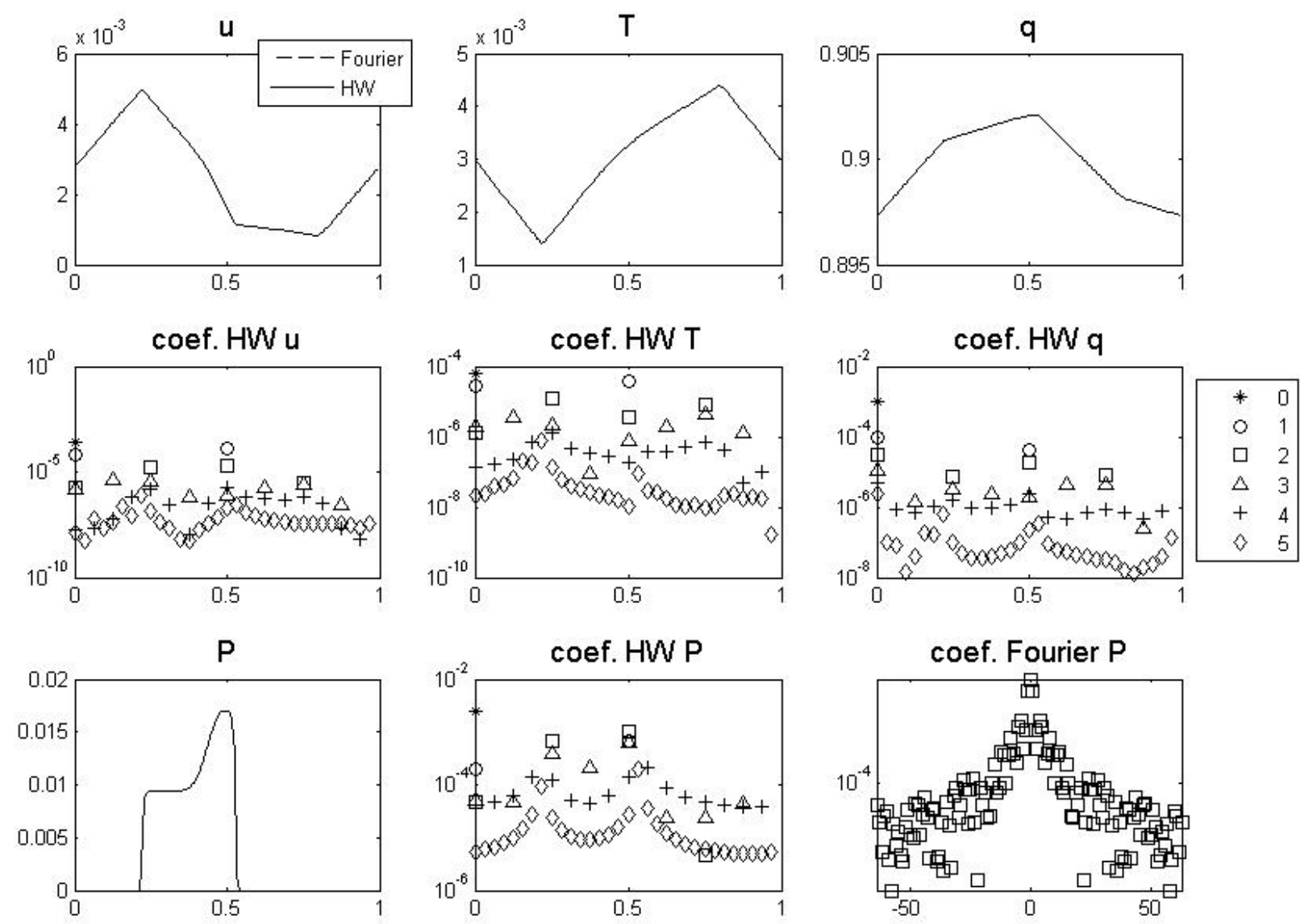

Parâmetros: Tempo=0.30 N=128 qhat $=0.9 \quad Q=0.9 \quad \alpha=0.6 \quad \tau=0.00625$ Dying front sem descont.

Figura 5.4: Frente atenuante no tempo 0.3 para as condições iniciais dadas nas equações 5.11. Os gráficos indicados por "coef." mostram os coeficientes de ondaletas harmônicas (HW) em módulo divididos por níveis. Os campos foram transladados em 0.5 no eixo $x$ para facilitar visualização das frentes. 

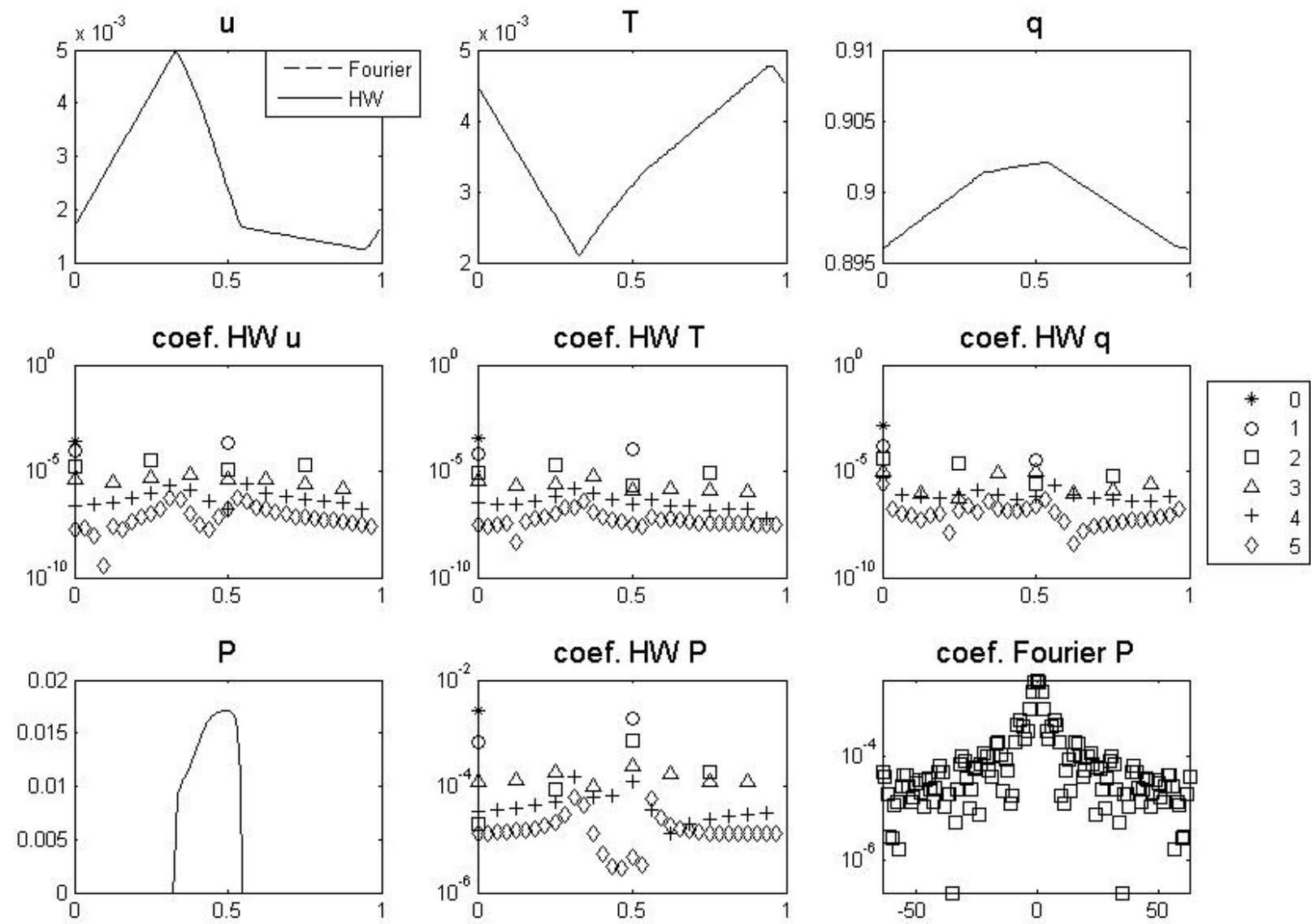

Parâmetros: Tempo=0.45 N=128 qhat =0.9 Q =0.9 $\alpha=0.6 \tau=0.00625$ Dying front sem descont.

Figura 5.5: Frente atenuante no tempo 0.45 para as condições iniciais dadas nas equações 5.11. Os gráficos indicados por "coef." mostram os coeficientes de ondaletas harmônicas (HW) em módulo divididos por níveis. Os campos foram transladados em 0.5 no eixo $x$ para facilitar visualização das frentes. 

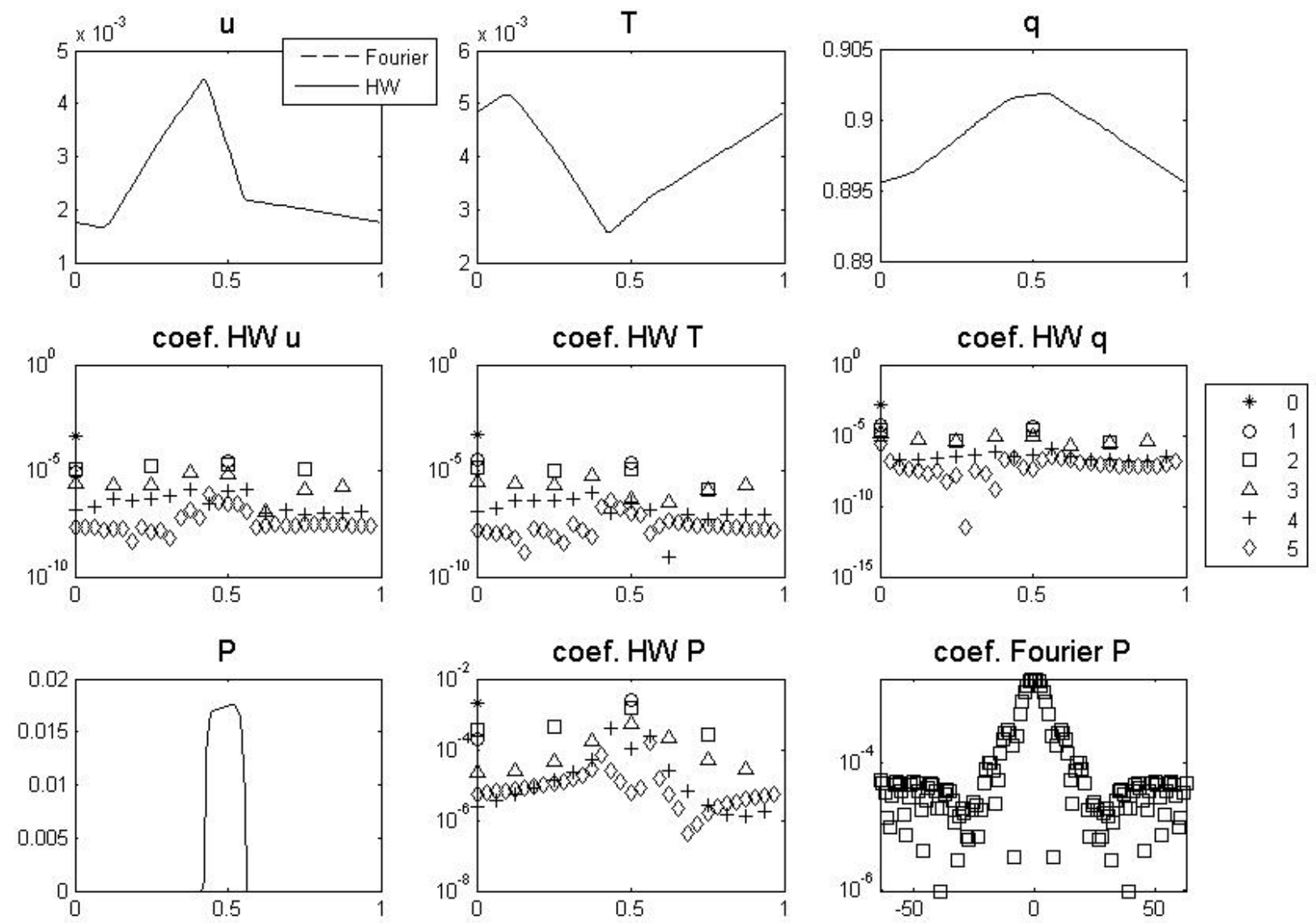

Parâmetros: Tempo=0.60 N=128 qhat =0.9 Q =0.9 $\alpha=0.6 \quad \tau=0.00625$ Dying front sem descont.

Figura 5.6: Frente atenuante no tempo 0.6 para as condições iniciais dadas nas equações 5.11. Os gráficos indicados por "coef." mostram os coeficientes de ondaletas harmônicas (HW) em módulo divididos por níveis. Os campos foram transladados em 0.5 no eixo $x$ para facilitar visualização das frentes. 

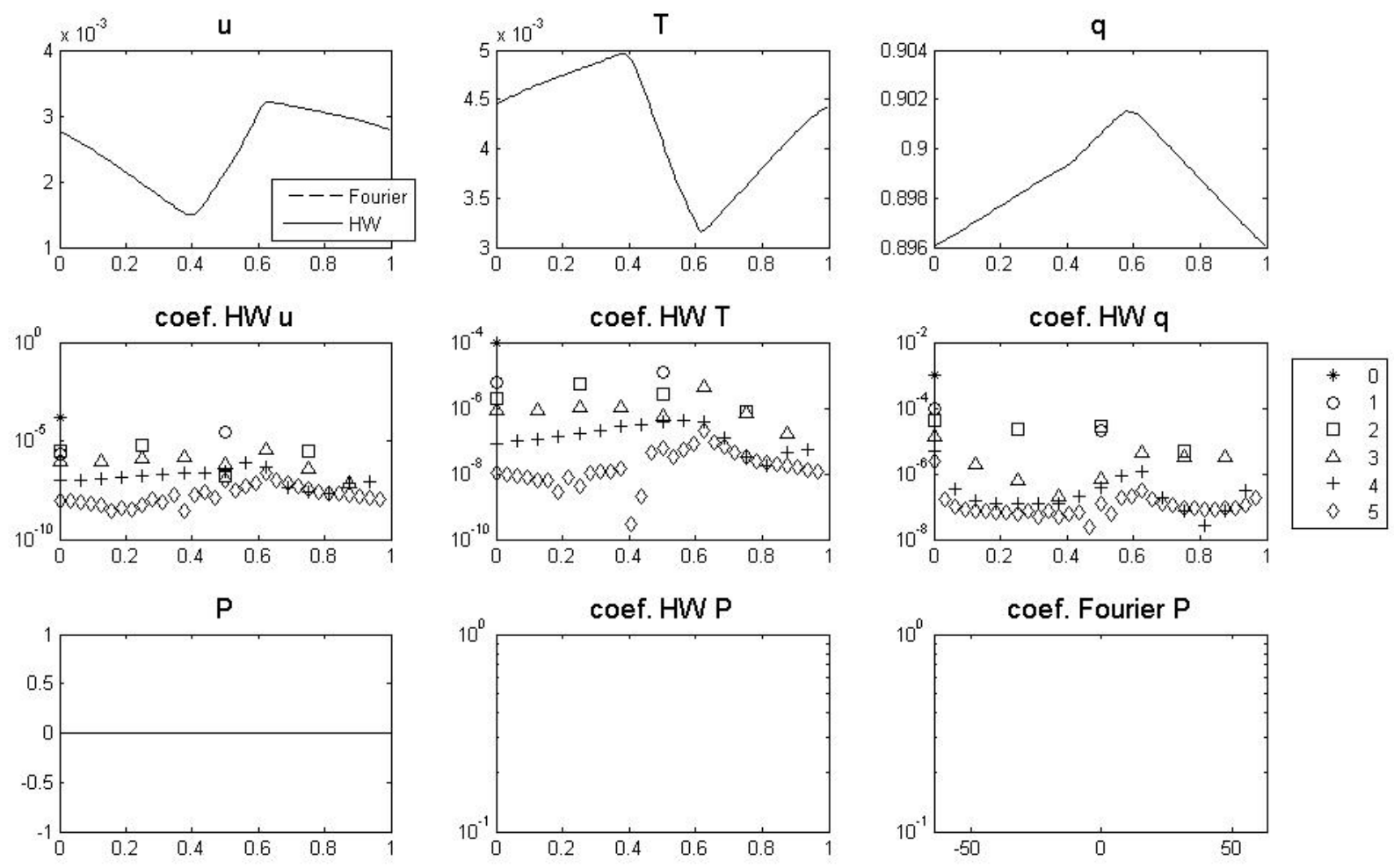

Parâmetros: Tempo=0.90 N=128 qhat $=0.9 \quad Q=0.9 \alpha=0.6 \tau=0.00625$ Dying front sem descont.

Figura 5.7: Frente atenuante no tempo 0.9 para as condições iniciais dadas nas equações 5.11. Os gráficos indicados por "coef." mostram os coeficientes de ondaletas harmônicas (HW) em módulo divididos por níveis. Os campos foram transladados em 0.5 no eixo $x$ para facilitar visualização das frentes. 

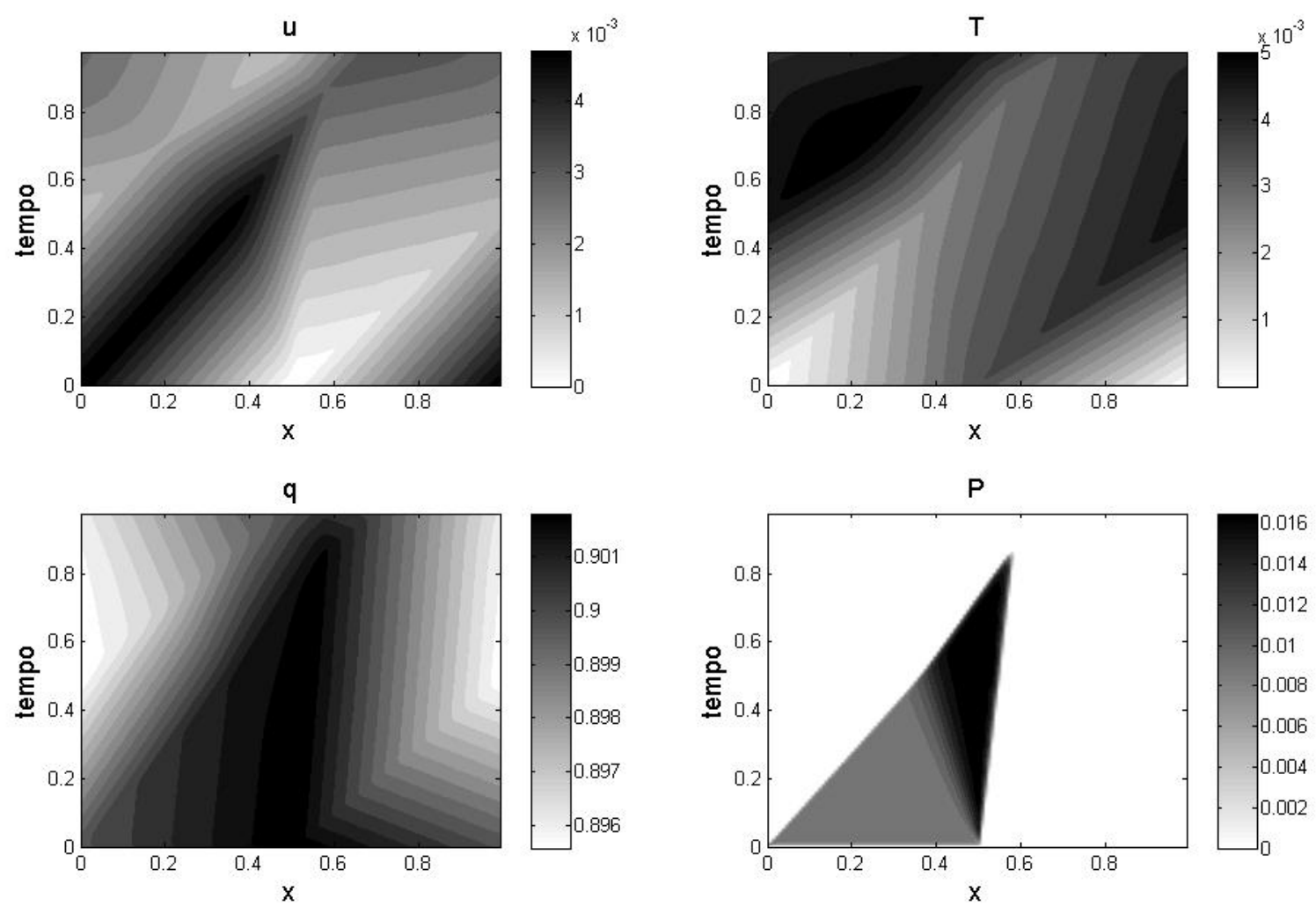

Parâmetros: Tempo=0.97656 N=128 qhat $=0.9 \quad Q=0.9 \quad \alpha=0.6 \quad \tau=0.00625$ Dying front sem descont.

Figura 5.8: Frente atenuante ao longo do tempo (no eixo y) para as condições iniciais dadas nas equações 5.11 Os gráficos mostram o valor do campo por intensidade de cor na escala cinza a cada instante de tempo para o intervalo unitário. Os campos foram transladados em 0.5 no eixo $x$ para facilitar visualização das frentes. 

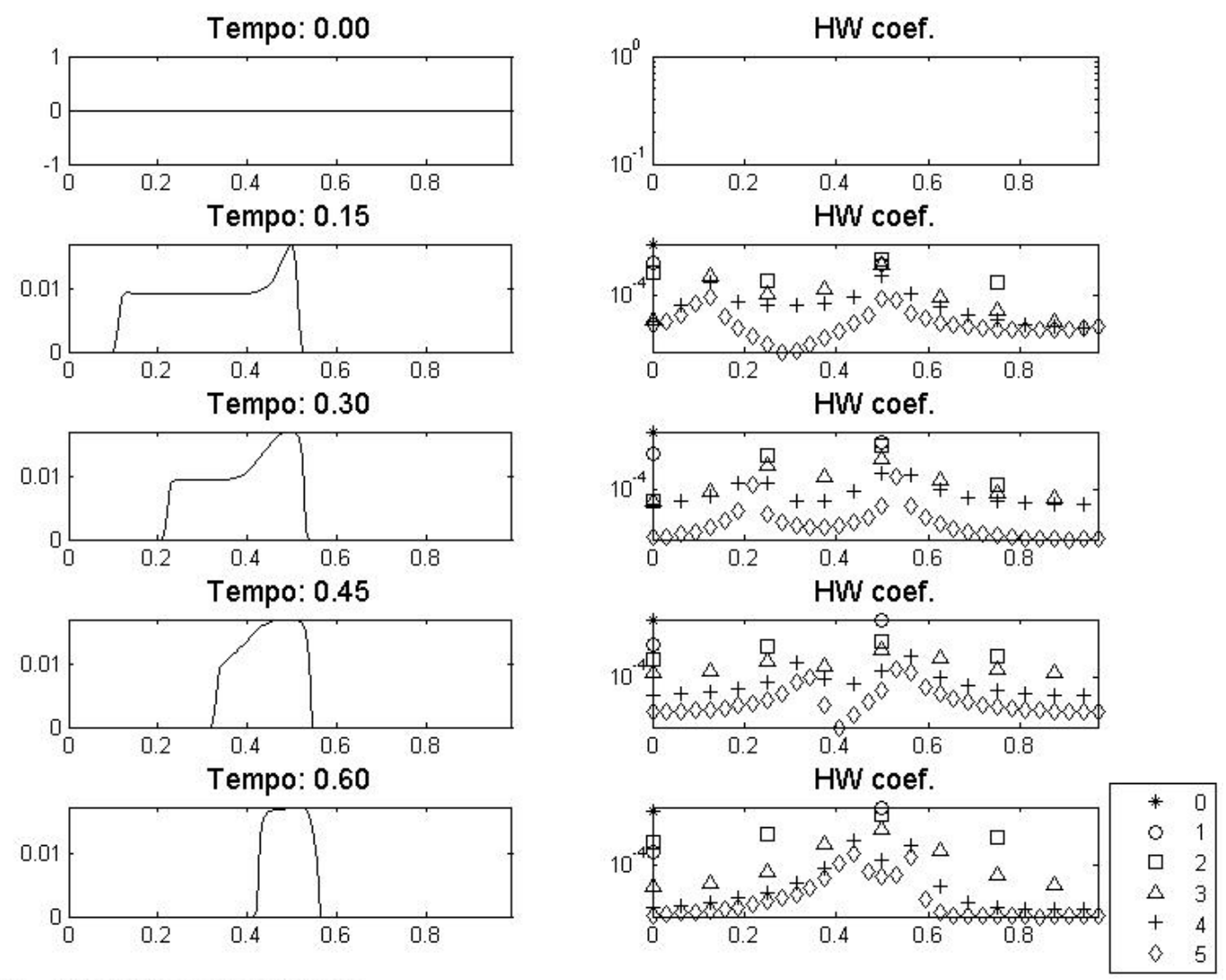

$\mathrm{N}=128$ Dying front sem descont.

Figura 5.9: Precipitação para frente atenuante ao longo do tempo para as condições iniciais dadas nas equações 5.11. Os gráficos mostram o valor do campo de precipitação e o os coeficientes de ondaletas harmônicas para alguns instantes de tempo no intervalo unitário. 
Vejamos o que ocorre ao considerarmos medidas de redução de custo computacional no método de Galerkin com ondaletas harmônicas, isto é, tomando-se cortes por localidade da base $(d>0)$. Consideramos o efeito de um corte com parâmetro $d=1$ em alguns instantes de tempo, que é apresentado na figura 5.10. Nota-se pouca diferença em relação ao caso sem corte por localidade (figura 5.9), e preserva-se a forma geral da solução, mas com um algoritmo com custo computacional muito menor. Testamos ainda o caso onde $d=3$, para o qual vamos analisar um nível a mais de ondaletas harmônicas, e portanto tomamos $\mathrm{N}=256$. Mostramos na figura 5.11 os resultados, onde nota-se claramente os picos dos coeficientes nos locais de frentes de precipitação. Por exemplo, em destaque na figura 5.12, no tempo 0.3, temos como elemento de maior valor no nível 5 o de translação $k=7$, e no nível 6 o de translação $k=14$, que referem-se à posição da frente desumidificante, seca, que se encontra no momento sobre o valor $7 / 32=14 / 64 \approx 0.22$ do intervalo unitário, e que condiz com a real posição da frente. $\mathrm{O}$ mesmo pode ser visto com relação à frente umidificante lenta, com posição no espaço dada por $17 / 32=34 / 64 \approx 0.53$ de acordo com ambos os níveis 5 e 6 . Contudo temos então um método que resolve as equações e ainda nos fornece tempo a tempo um método para localizar as frentes de precipitação no espaço.
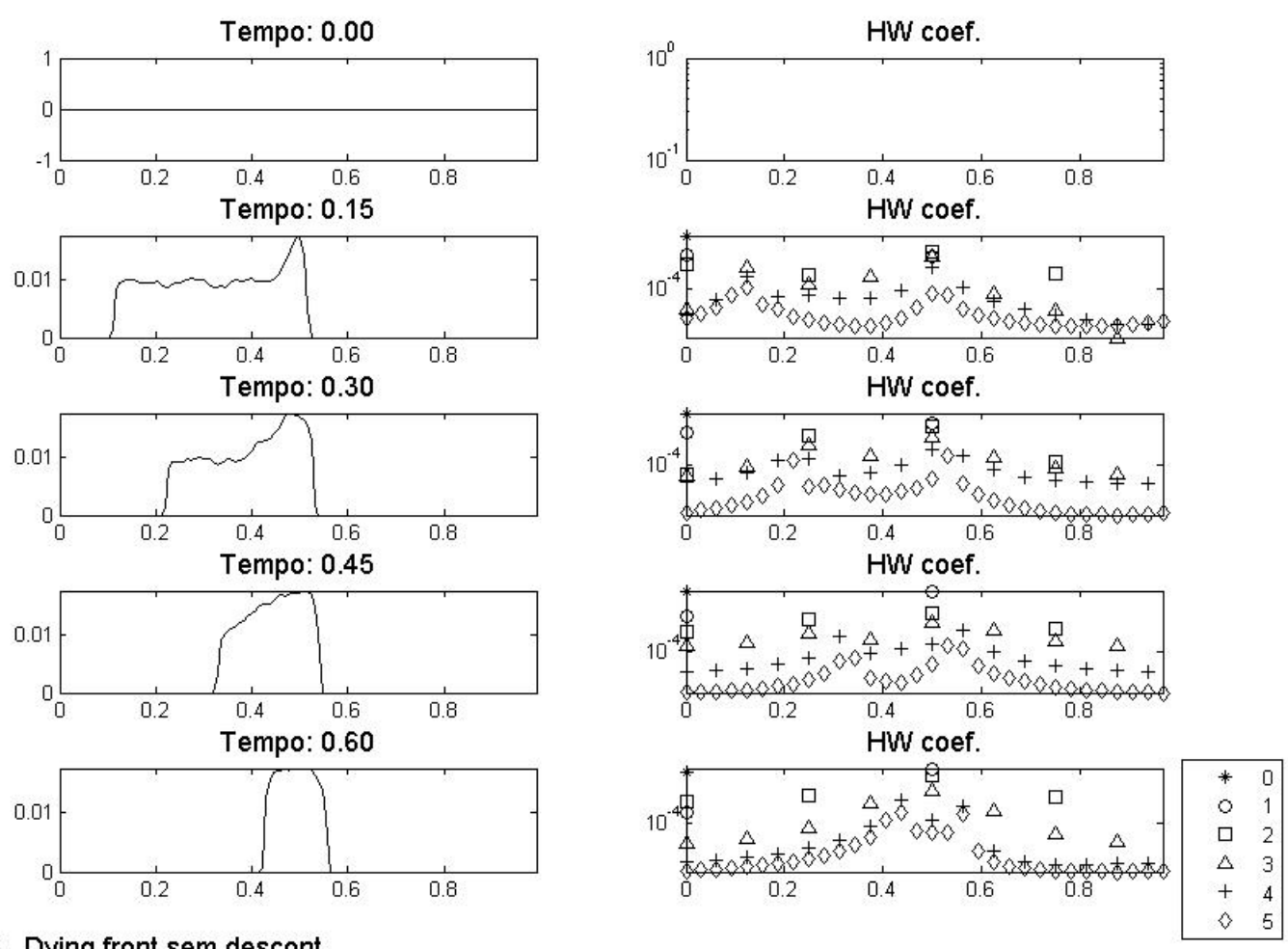

$N=128$ Dying front sem descont.

Figura 5.10: Precipitação para frente atenuante ao longo do tempo para as condições iniciais dadas nas equações 5.11 considerando corte por localidade $d=1$. Os gráficos mostram o valor do campo de precipitação e o os coeficientes de ondaletas harmônicas para alguns instantes de tempo no intervalo unitário. 

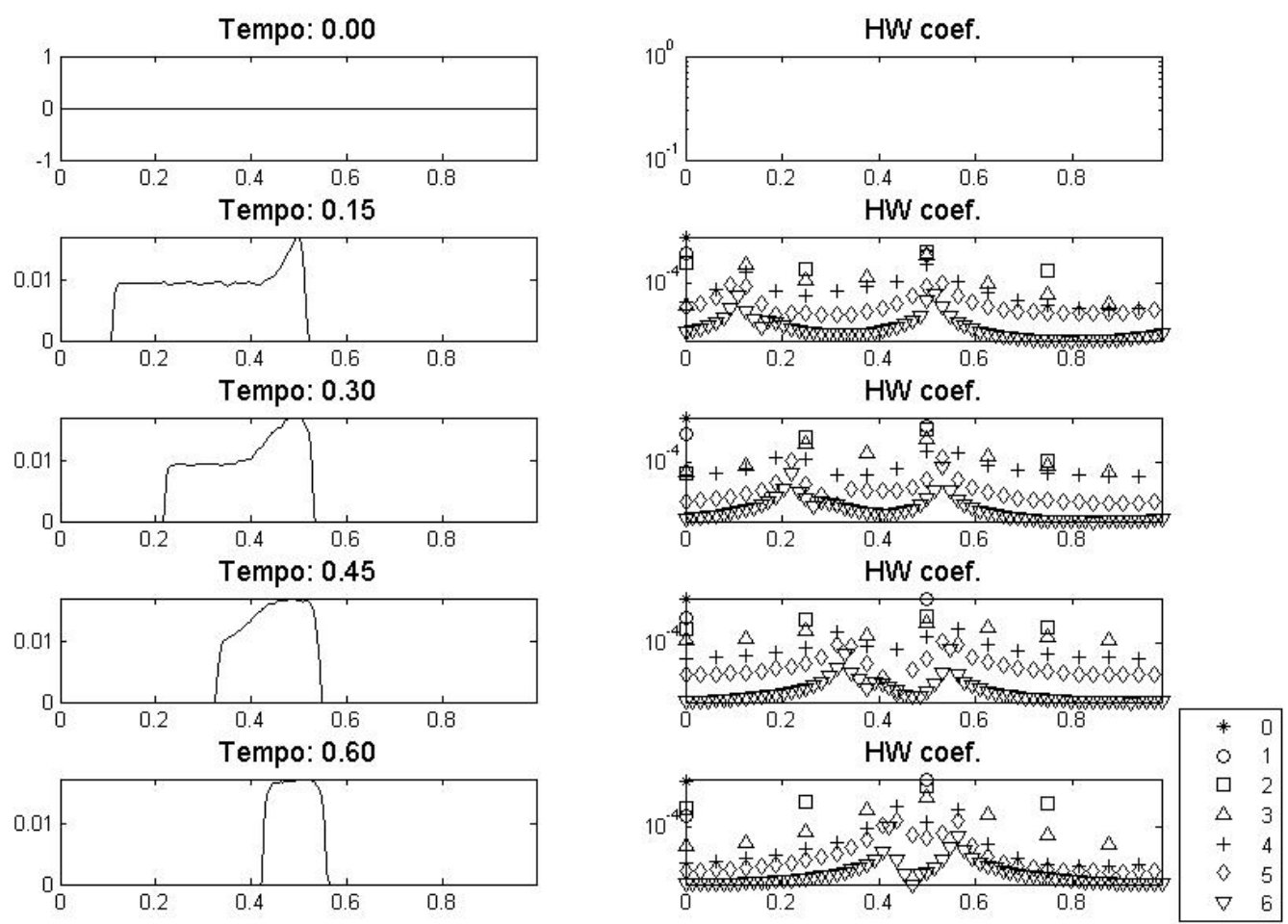

$N=256$ Dying front sem descont.

Figura 5.11: Precipitação para frente atenuante ao longo do tempo para as condições iniciais dadas nas equações 5.11 considerando corte por localidade $d=3$. Os gráficos mostram o valor do campo de precipitação e o os coeficientes de ondaletas harmônicas para alguns instantes de tempo no intervalo unitário. 
HW coef. no tempo 0.3

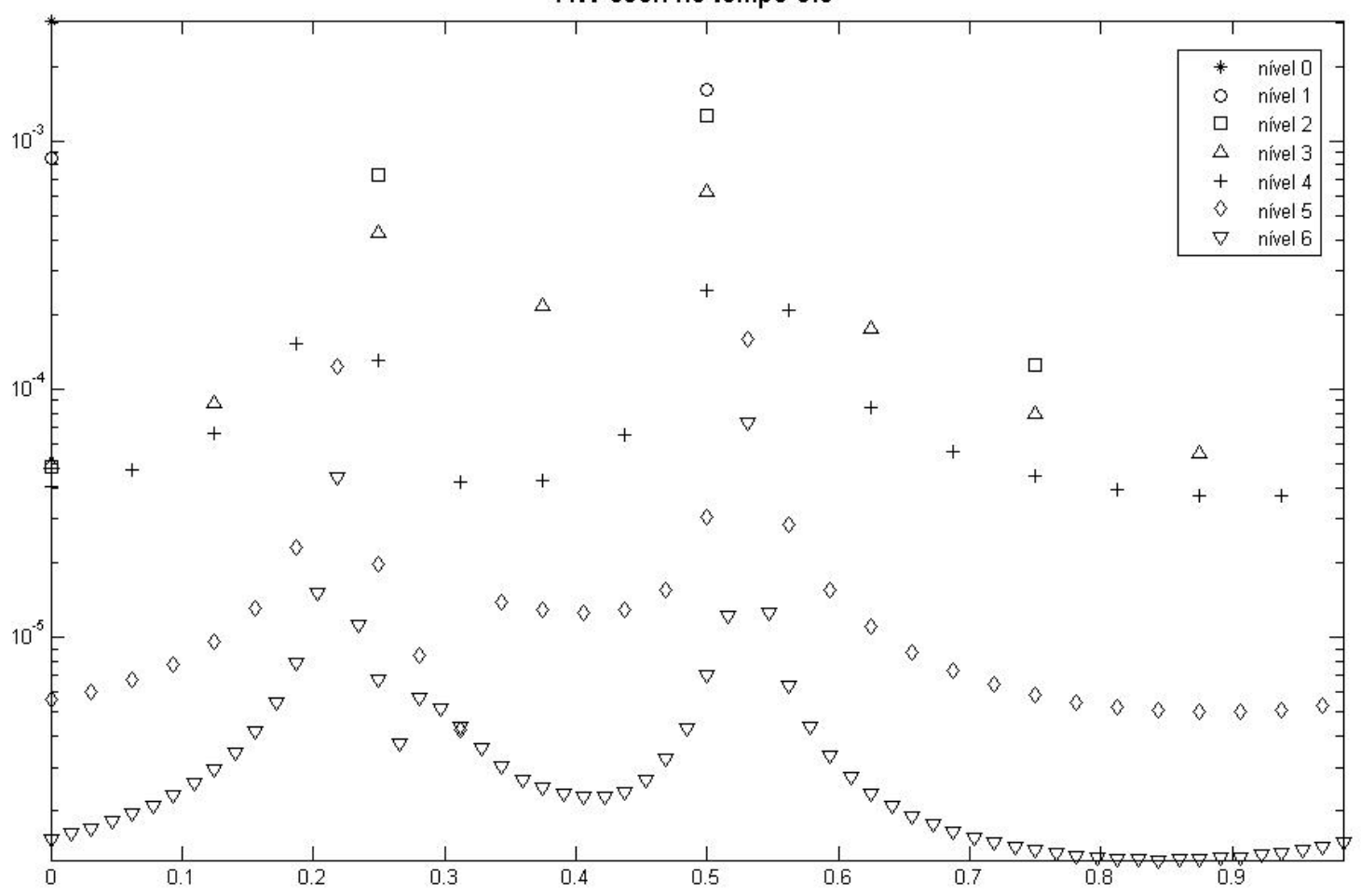

$\mathrm{N}=256$ Dying front sem descont.

Figura 5.12: Coeficientes de ondaletas harmônicas para precipitação da frente atenuante ao no tempo 0.3 para as condições iniciais dadas nas equações 5.11 considerando corte por localidade $d=3$. 


\section{Capítulo 6}

\section{Conclusões}

Muito vem sendo estudado na direção de se utilizar ondaletas em métodos de resolução de equações diferenciais. Tentamos neste estudo analisar mais a fundo questões teóricas e numéricas ligadas à resolução de EDPs com métodos de Galerkin com base de ondaletas harmônicas. Foram investigados em particular casos unidimensionais de equações de advecção, linear ou não linear, resolvidas com um método de Galerkin com base de ondaletas harmônicas. Para tanto foi necessário analisar detalhadamente as propriedades das ondaletas harmônicas. Ilustramos a proximidade das ondaletas harmônicas com o conjunto de funções trigonométricas de Fourier, e sua propriedade de localidade no espaço e no domínio de frequências, demonstrando a possibilidade de se usar estas ondaletas para aproximar funções do $L^{2}([0,1])$. Ao compreendermos as características da base, foi possível deduzir as equações de um método numérico de Galerkin, que foi considerado para uma bateria de experimentos numéricos.

O método de Galerkin com base de ondaletas harmônicas mostrou-se muito similar ao método de Galerkin com base de Fourier. Ao passo que as ondaletas harmônicas encarecem computacionalmente o método, há em contrapartida o ganho de termos informações sobre a localidade do sinal no domínio espectral. Nos casos lineares é possível obtermos um custo computacional da mesma ordem daquele obtido com o método usando base de Fourier, se considerarmos as características de localidade no espaço da base de ondaletas. Porém no caso não linear há um grande acréscimo de custo computacional devido aos cálculos dos coeficientes de conexão, e consequente grande quantidade de cômputos necessários para se avaliar o sistema gerado. Comparando com um método de Galerkin com base de Fourier é possível obtermos custo computacional semelhante com ondaletas harmônicas, porém este custo ainda é alto quando comparado com outras alternativas disponíveis na literatura (por exemplo um método pseudo-espectral com base de Fourier). É de se esperar que outras bases de ondaletas sem suporte compacto (por exemplo as ondaletas de Meyer LOPES; MATTOS, 2003)) venham a compartilhar deste mesmo resultado, assim como para ondaletas com suporte compacto, conforme descrito, por exemplo, em Schult e Wyld (1992). Por outro lado, ao analisarmos o método pseudo-espectral com base de ondaletas harmônicas, preservamos a boa aproximação com a solução esperada e as características de localidade da base, mas reduzimos significativamente o custo computacional.

Quanto aos testes do método em relação ao modelo de propagação de frente de precipitação, 
o mesmo mostrou resultados que condizem com a solução esperada do fenômeno físico. Ao considerarmos restrições do suporte das ondaletas harmônicas $(d=1$ ou $d=3)$ temos um custo computacional ótimo $(O(N))$, com praticamente nenhuma perda de informações relevantes sobre o sinal. Além disso o método proporciona a possibilidade de se obter informações sobre a localidade do sinal, ou de frentes no sinal, espectralmente, isto é, olhando apenas para os coeficientes de ondaletas.

Contudo utilizar ondaletas harmônicas na resolução de EDPs através de um método de Galerkin pode ser interessante do ponto de vista de estudos de sinais bem localizados, como frentes de propagação. Por outro lado, requer um alto custo computacional, que pode ser reduzido em componentes não lineares utilizando um método pseudo-espectral, e em componentes lineares utilizando a característica de localidade da base de ondaletas. 


\section{Apêndice A}

\section{Cálculos da projeção de não linearidades com derivadas na base de ondaletas harmônicas}

Queremos estudar a projeção da função $w=v u_{x}$ na base de ondaletas harmônicas, com $v$ e $u$ escritas na base de ondaletas harmônicas,

$$
\begin{aligned}
v(x)= & a_{0}+\sum_{j=0}^{n-2} \sum_{k=0}^{2^{j}-1}\left(a_{2^{j}+k} \psi_{j, k}(x)+a_{2^{j}+k}^{*} \psi_{j, k}^{*}(x)\right)+a_{N / 2} \psi_{n-1}(x), \\
u_{x}= & \sum_{p=0}^{n-2} \sum_{q=0}^{2^{p}-1}\left(b_{2^{p}+q} \frac{d \psi_{p, q}(x)}{d x}+b_{2^{p}+q}^{*} \frac{d \psi_{p, q}^{*}(x)}{d x}\right)+b_{N / 2} \frac{d \psi_{n-1}(x)}{d x}, \\
w(x)= & \left(a_{0}+\sum_{j=0}^{n-2} \sum_{k=0}^{2^{j}-1}\left(a_{2^{j}+k} \psi_{j, k}(x)+a_{2^{j}+k}^{*} \psi_{j, k}^{*}(x)\right)+a_{N / 2} \psi_{n-1}(x)\right) \\
& \left(\sum_{p=0}^{n-2} \sum_{q=0}^{2^{p}-1}\left(b_{2^{p}+q} \frac{d \psi_{p, q}(x)}{d x}+b_{2^{p}+q}^{*} \frac{d \psi_{p, q}^{*}(x)}{d x}\right)+b_{N / 2} \frac{d \psi_{n-1}(x)}{d x}\right) \\
= & (A)+(B)+(C)+(D),
\end{aligned}
$$

onde,

$$
\begin{aligned}
(A) & =\left(a_{0}\right)\left(\sum_{p=0}^{n-2} \sum_{q=0}^{2^{p}-1}\left(b_{2^{p}+q} \frac{d \psi_{p, q}(x)}{d x}+b_{2^{p}+q}^{*} \frac{d \psi_{p, q}^{*}(x)}{d x}\right)+b_{N / 2} \frac{d \psi_{n-1}(x)}{d x}\right) \\
(B)= & \left(\sum_{j=0}^{n-2} \sum_{k=0}^{2^{j}-1} a_{2^{j}+k} \psi_{j, k}(x)\right)\left(\sum_{p=0}^{n-2} \sum_{q=0}^{2^{p}-1}\left(b_{2^{p}+q} \frac{d \psi_{p, q}(x)}{d x}+b_{2^{p}+q}^{*} \frac{d \psi_{p, q}^{*}(x)}{d x}\right)+b_{N / 2} \frac{d \psi_{n-1}(x)}{d x}\right) \\
(C)= & \left(\sum_{j=0}^{n-2} \sum_{k=0}^{2^{j}-1} a_{2^{j}+k}^{*} \psi_{j, k}^{*}(x)\right)\left(\sum_{p=0}^{n-2} \sum_{q=0}^{2^{p}-1}\left(b_{2^{p}+q} \frac{d \psi_{p, q}(x)}{d x}+b_{2^{p}+q}^{*} \frac{d \psi_{p, q}^{*}(x)}{d x}\right)+b_{N / 2} \frac{d \psi_{n-1}(x)}{d x}\right) \\
(D)= & \left(a_{N / 2} \psi_{n-1}(x)\right)\left(\sum_{p=0}^{n-2} \sum_{q=0}^{2^{p}-1}\left(b_{2^{p}+q} \frac{d \psi_{p, q}(x)}{d x}+b_{2^{p}+q}^{*} \frac{d \psi_{p, q}^{*}(x)}{d x}\right)+b_{N / 2} \frac{d \psi_{n-1}(x)}{d x}\right)
\end{aligned}
$$


Vamos estudar cada parcela separadamente. A parcela $(A)$ é equivalente ao desenvolvido nas seções anteriores sobre projeção das derivadas de uma função na base de ondaletas harmônicas. Portanto, desta parcela, $(A)$, vamos ter:

$$
\begin{aligned}
\langle(A), \phi\rangle & =0 \\
\left\langle(A), \psi_{r, s}(x)\right\rangle & =a_{0} \sum_{q=0}^{2^{r}-1} b_{2^{r}+q} \gamma_{q s}^{r} \\
\left\langle(A), \psi_{n-1}(x)\right\rangle & =-N \pi i a_{0} b_{N / 2} .
\end{aligned}
$$

Na parcela $(B)$ começam a surgir novos coeficientes de conexão, vejamos,

$$
\begin{aligned}
(B) & =\left(\sum_{j=0}^{n-2} \sum_{k=0}^{2^{j}-1} a_{2^{j}+k} \psi_{j, k}(x)\right)\left(\sum_{p=0}^{n-2} \sum_{q=0}^{2^{p}-1}\left(b_{2^{p}+q} \frac{d \psi_{p, q}(x)}{d x}+b_{2^{p}+q}^{*} \frac{d \psi_{p, q}^{*}(x)}{d x}\right)+b_{N / 2} \frac{d \psi_{n-1}(x)}{d x}\right) \\
& =\sum_{j=0}^{n-2} \sum_{k=0}^{2^{j}-1} \sum_{p=0}^{n-2} \sum_{q=0}^{2^{p}-1}\left(a_{2^{j}+k} b_{2^{p}+q} \psi_{j, k}(x) \frac{d \psi_{p, q}(x)}{d x}\right) \quad(B 1) \\
& +\sum_{j=0}^{n-2} \sum_{k=0}^{2^{j}-1} \sum_{p=0}^{n-2} \sum_{q=0}^{2^{p}-1}\left(a_{2^{j}+k} b_{2^{p}+q}^{*} \psi_{j, k}(x) \frac{d \psi_{p, q}^{*}(x)}{d x}\right) \quad(B 2) \\
& +\sum_{j=0}^{n-2} \sum_{k=0}^{2^{j}-1}\left(a_{2^{j}+k} b_{N / 2} \psi_{j, k}(x) \frac{d \psi_{n-1}(x)}{d x}\right) \quad(B 3)
\end{aligned}
$$

Quando a expressão acima é projetada na base teremos diversos coeficientes de conexão. Omitiremos passagens mais simples e que apresentaremos a seguir os coeficientes de conexão pertinentes.

1. (B1)
(a) $\left\langle\psi_{j, k}(x) \frac{d \psi_{p, q}(x)}{d x}, 1\right\rangle=0$,
(b) $\left\langle\psi_{j, k}(x) \frac{d \psi_{p, q}(x)}{d x}, \psi_{r, s}(x)\right\rangle=P(0)_{k q s}^{j p r}$,
(c) $\left\langle\psi_{j, k}(x) \frac{d \psi_{p, q}(x)}{d x}, \psi_{n-1}(x)\right\rangle=0$,

2. (B2)

(a) $\left\langle\psi_{j, k}(x) \frac{d \psi_{p, q}^{*}(x)}{d x}, 1\right\rangle=\left(\gamma_{q k}^{j}\right)^{*}$, somente se $j=p$, senão é nulo,

(b) $\left\langle\psi_{j, k}(x) \frac{d \psi_{p, q}^{*}(x)}{d x}, \psi_{r, s}(x)\right\rangle=P(2)_{k q s}^{j p r}$,

(c) $\left\langle\psi_{j, k}(x) \frac{d \psi_{p, q}^{*}(x)}{d x}, \psi_{n-1}(x)\right\rangle=0$,

3. (B3)

(a) $\left\langle\psi_{j, k}(x) \frac{d \psi_{n-1}(x)}{d x}, 1\right\rangle=0$, 
(b) $\left\langle\psi_{j, k}(x) \frac{d \psi_{n-1}(x)}{d x}, \psi_{r, s}(x)\right\rangle=0$,

(c) $\left\langle\psi_{j, k}(x) \frac{d \psi_{n-1}(x)}{d x}, \psi_{n-1}(x)\right\rangle=0$.

Onde $\gamma_{q k}^{j}$ é o coeficiente de conexão linear dado por 3.40 , e os coeficientes de conexão não lineares são dados por

$$
\begin{gathered}
P(0)_{k q s}^{j p r}=\frac{2 \pi i}{2^{(j+p+r) / 2}} \sum_{m_{j}=2^{j}}^{2^{j+1}-1} \sum_{m_{p}=\max \left(2^{r}-m_{j}, 2^{p}\right)}^{\min \left(2^{r+1}-1-m_{j}, 2^{p+1}-1\right)} m_{p} e^{-2 \pi i\left(\frac{m_{j} k}{2^{j}}+\frac{m_{p} q}{2^{p}}-\frac{\left(m_{j}+m_{p}\right) s}{2^{r}}\right)}, \\
P(2)_{k q s}^{j p r}=-\frac{2 \pi i}{2^{(j+p+r) / 2}} \sum_{m_{j}=2^{j}}^{2^{j+1}-1} \sum_{m_{p}=\max \left(m_{j}-\left(2^{r+1}-1\right), 2^{p}\right)}^{\min \left(m_{j}-2^{r}, 2^{p+1}-1\right)} m_{p} e^{-2 \pi i\left(\frac{m_{j} k}{2^{j}}-\frac{m_{p} q}{2^{p}}-\frac{\left(m_{j}-m_{p}\right) s}{2^{r}}\right)},
\end{gathered}
$$

Com isso ficamos com a projeção de (B) na base de ondaletas harmônicas dada por:

$$
\begin{aligned}
\langle(B), \phi\rangle & =\sum_{j=0}^{n-2} \sum_{k=0}^{2^{j}-1} \sum_{q=0}^{2^{j}-1}\left(a_{2^{j}+k} b_{2^{j}+q}^{*}\left(\gamma_{q k}^{j}\right)^{*}\right) \\
\left\langle(B), \psi_{r, s}(x)\right\rangle & =\sum_{j=0}^{n-2} \sum_{k=0}^{2^{j}-1} \sum_{p=0}^{n-2} \sum_{q=0}^{2^{p}-1}\left(a_{2^{j}+k} b_{2^{p}+q} P(0)_{k q s}^{j p r}+a_{2^{j}+k} b_{2^{p}+q}^{*} P(2)_{k q s}^{j p r}\right) \\
\left\langle(B), \psi_{n-1}(x)\right\rangle & =0 .
\end{aligned}
$$

Vamos olhar agora para a parcela $(C)$.

$$
\begin{aligned}
(C) & =\left(\sum_{j=0}^{n-2} \sum_{k=0}^{2^{j}-1} a_{2^{j}+k}^{*} \psi_{j, k}^{*}(x)\right)\left(\sum_{p=0}^{n-2} \sum_{q=0}^{2^{p}-1}\left(b_{2^{p}+q} \frac{d \psi_{p, q}(x)}{d x}+b_{2^{p}+q}^{*} \frac{d \psi_{p, q}^{*}(x)}{d x}\right)+b_{N / 2} \frac{d \psi_{n-1}(x)}{d x}\right) \\
& =\sum_{j=0}^{n-2} \sum_{k=0}^{2^{j}-1} \sum_{p=0}^{n-2} \sum_{q=0}^{2^{p}-1} a_{2^{j}+k}^{*} b_{2^{p}+q} \psi_{j, k}^{*}(x) \frac{d \psi_{p, q}(x)}{d x} \quad(C 1) \\
& +\sum_{j=0}^{n-2} \sum_{k=0}^{2^{j}-1} \sum_{p=0}^{n-2} \sum_{q=0}^{2^{p}-1} a_{2^{j}+k}^{*} b_{2^{p}+q}^{*} \psi_{j, k}^{*}(x) \frac{d \psi_{p, q}^{*}(x)}{d x} \quad(C 2) \\
& +\sum_{j=0}^{n-2} \sum_{k=0}^{2^{j}-1} a_{2^{j}+k}^{*} b_{N / 2} \psi_{j, k}^{*}(x) \frac{d \psi_{n-1}(x)}{d x}(C 3)
\end{aligned}
$$

com isso teremos que avaliar os produtos internos,

1. $(\mathrm{C} 1)$

(a) $\left\langle\psi_{j, k}^{*}(x) \frac{d \psi_{p, q}(x)}{d x}, 1\right\rangle=\gamma_{q k}^{j}$, somente se $j=p$, senão é nulo,

(b) $\left\langle\psi_{j, k}^{*}(x) \frac{d \psi_{p, q}(x)}{d x}, \psi_{r, s}(x)\right\rangle=P(1)_{k q s}^{j p r}$, 
(c) $\left\langle\psi_{j, k}^{*}(x) \frac{d \psi_{p, q}(x)}{d x}, \psi_{n-1}(x)\right\rangle=0$,

2. $(\mathrm{C} 2)$

(a) $\left\langle\psi_{j, k}^{*}(x) \frac{d \psi_{p, q}^{*}(x)}{d x}, 1\right\rangle=0$,

(b) $\left\langle\psi_{j, k}^{*}(x) \frac{d \psi_{p, q}^{*}(x)}{d x}, \psi_{r, s}(x)\right\rangle=0$,

(c) $\left\langle\psi_{j, k}^{*}(x) \frac{d \psi_{p, q}^{*}(x)}{d x}, \psi_{n-1}(x)\right\rangle=N_{k q}^{j p *}$, será não nulo somente se $j=n-2$ ou $p=n-2$.

3. (C3)

(a) $\left\langle\psi_{j, k}^{*}(x) \frac{d \psi_{n-1}(x)}{d x}, 1\right\rangle=0$,

(b) $\left\langle\psi_{j, k}^{*}(x) \frac{d \psi_{n-1}(x)}{d x}, \psi_{r, s}(x)\right\rangle=0$,

(c) $\left\langle\psi_{j, k}^{*}(x) \frac{d \psi_{n-1}(x)}{d x}, \psi_{n-1}(x)\right\rangle=0$.

Onde,

$$
\begin{gathered}
P(1)_{k q s}^{j p r}=\frac{2 \pi i}{2^{(j+p+r) / 2}} \sum_{m_{j}=2^{j}}^{2^{j+1}-1} \sum_{m_{p}=\max \left(2^{r}+m_{j}, 2^{p}\right)}^{\min \left(2^{r+1}-1+m_{j}, 2^{p+1}-1\right)} m_{p} e^{-2 \pi i\left(-\frac{m_{j} k}{2^{j}}+\frac{m_{p} q}{2^{p}}-\frac{\left(m_{p}-m_{j}\right) s}{2^{r}}\right)}, \\
N_{k q}^{j p}=\frac{2 \pi i}{2^{(j+p) / 2}} \sum_{m_{p}=\max \left(N / 2-\left(2^{j+1}-1\right), 2^{p}\right)}^{\min \left(N / 2-2^{j}, 2^{p+1}-1\right)} m_{p} e^{2 \pi i m_{p}\left(\frac{k}{2^{j}}-\frac{q}{2^{p}}\right)} .
\end{gathered}
$$

Resultando uma projeção para parcela (C) da seguinte forma,

$$
\begin{aligned}
\langle(C), \phi\rangle & =\sum_{j=0}^{n-2} \sum_{k=0}^{2^{j}-1} \sum_{q=0}^{2^{j}-1}\left(a_{2^{j}+k}^{*} b_{2^{j}+q}\left(\gamma_{q k}^{j}\right)\right) \\
\left\langle(C), \psi_{r, s}(x)\right\rangle & =\sum_{j=0}^{n-2} \sum_{k=0}^{2^{j}-1} \sum_{p=0}^{n-2} \sum_{q=0}^{2^{p}-1}\left(a_{2^{j}+k}^{*} b_{2^{p}+q} P(1)_{k q s}^{j p r}\right) \\
\left\langle(C), \psi_{n-1}(x)\right\rangle & =\sum_{j=0}^{n-2} \sum_{k=0}^{2^{j}-1} \sum_{p=0}^{n-2} \sum_{q=0}^{2^{p}-1} a_{2^{j}+k}^{*} b_{2^{p}+q}^{*} N_{k q}^{j p *} .
\end{aligned}
$$


Para finalizar os cálculos vamos ao caso $(D)$,

$$
\begin{aligned}
(D) & =\left(a_{N / 2} \psi_{n-1}(x)\right)\left(\sum_{p=0}^{n-2} \sum_{q=0}^{2^{p}-1}\left(b_{2^{p}+q} \frac{d \psi_{p, q}(x)}{d x}+b_{2^{p}+q}^{*} \frac{d \psi_{p, q}^{*}(x)}{d x}\right)+b_{N / 2} \frac{d \psi_{n-1}(x)}{d x}\right) \\
& =\sum_{p=0}^{n-2} \sum_{q=0}^{2^{p}-1} a_{N / 2} b_{2^{p}+q} \psi_{n-1}(x) \frac{d \psi_{p, q}(x)}{d x} \quad(D 1) \\
& +\sum_{p=0}^{n-2} \sum_{q=0}^{2^{p}-1} a_{N / 2} b_{2^{p}+q}^{*} \psi_{n-1}(x) \frac{d \psi_{p, q}^{*}(x)}{d x} \quad(D 2) \\
& +a_{N / 2} b_{N / 2} \psi_{n-1}(x) \frac{d \psi_{n-1}(x)}{d x} \quad(D 3),
\end{aligned}
$$

resultando nos coeficientes de conexão,

1. (D1)
(a) $\left\langle\psi_{n-1}(x) \frac{d \psi_{p, q}(x)}{d x}, 1\right\rangle=0$,
(b) $\left\langle\psi_{n-1}(x) \frac{d \psi_{p, q}(x)}{d x}, \psi_{r, s}(x)\right\rangle=0$,
(c) $\left\langle\psi_{n-1}(x) \frac{d \psi_{p, q}(x)}{d x}, \psi_{n-1}(x)\right\rangle=0$,

2. (D2)
(a) $\left\langle\psi_{n-1}(x) \frac{d \psi_{p, q}^{*}(x)}{d x}, 1\right\rangle=0$,
(b) $\left\langle\psi_{n-1}(x) \frac{d \psi_{p, q}^{*}(x)}{d x}, \psi_{r, s}(x)\right\rangle=0$,
(c) $\left\langle\psi_{n-1}(x) \frac{d \psi_{p, q}^{*}(x)}{d x}, \psi_{n-1}(x)\right\rangle=0$,

3. (D3)
(a) $\left\langle\psi_{n-1}(x) \frac{d \psi_{n-1}(x)}{d x}, 1\right\rangle=-\pi i N$,
(b) $\left\langle\psi_{n-1}(x) \frac{d \psi_{n-1}(x)}{d x}, \psi_{r, s}(x)\right\rangle=0$,
(c) $\left\langle\psi_{n-1}(x) \frac{d \psi_{n-1}(x)}{d x}, \psi_{n-1}(x)\right\rangle=0$.

Resultando uma projeção para parcela (D) da seguinte forma,

$$
\begin{aligned}
\langle(D), \phi\rangle & =-N \pi i a_{N / 2} b_{N / 2} \\
\left\langle(D), \psi_{r, s}(x)\right\rangle & =0 \\
\left\langle(D), \psi_{n-1}(x)\right\rangle & =0 .
\end{aligned}
$$

Agora sabendo que $w(x)=A+B+C+D$, podemos obter a projeção de $w$ na base de ondaletas 
harmônicas.

$$
\begin{aligned}
\langle w, \phi\rangle & =\sum_{j=0}^{n-2} \sum_{k=0}^{2^{j}-1} \sum_{q=0}^{2^{j}-1}\left(a_{2^{j}+k} b_{2^{j}+q}^{*}\left(\gamma_{q k}^{j}\right)^{*}+a_{2^{j}+k}^{*} b_{2^{j}+q}\left(\gamma_{q k}^{j}\right)\right)-N \pi i a_{N / 2} b_{N / 2} \\
\left\langle w, \psi_{r, s}(x)\right\rangle & =a_{0} \sum_{q=0}^{2^{r}-1} b_{2^{r}+q} \gamma_{q s}^{r} \\
& +\sum_{j=0}^{n-2} \sum_{k=0} \sum_{p=0}^{2^{j}-1} \sum_{q=0}^{n-2}\left(a_{2^{j}+k} b_{2^{p}+q} P(0)_{k q s}^{j p r}+a_{2^{j}+k}^{*} b_{2^{p}+q} P(1)_{k q s}^{j p r}\right) \\
& +\sum_{j=0}^{n-2} \sum_{k=0}^{2^{j}-1} \sum_{p=0}^{n-2} \sum_{q=0}^{2^{p}-1}\left(a_{2^{j}+k} b_{2^{p}+q}^{*} P(2)_{k q s}^{j p r}\right) \\
\left\langle w, \psi_{n-1}(x)\right\rangle= & -N \pi i a_{0} b_{N / 2}+\sum_{j=0}^{n-2} \sum_{k=0}^{2^{j}-1} \sum_{p=0}^{n-2} \sum_{q=0}^{2^{p}-1}\left(a_{2^{j}+k}^{*} b_{2^{p}+q}^{*} N_{k q}^{j p *}\right)
\end{aligned}
$$




\section{Apêndice B}

\section{Transformada de Ondaletas Harmônicas para Derivadas}

Seja $f(x)$ uma função de $L^{2}([0,1])$ expandida em série de ondaletas harmônicas,

$$
f(x)=a_{0}+\sum_{j=0}^{n-2} \sum_{k=0}^{2^{j}-1}\left(a_{2^{j}+k} \psi_{j, k}(x)+\tilde{a}_{2^{j}+k} \psi_{j, k}^{*}(x)\right)+a_{N / 2} \psi_{n-1}(x),
$$

e sua derivada, $g(x)=\frac{d f(x)}{d x}$,

$$
g(x)=\sum_{j=0}^{n-2} \sum_{k=0}^{2^{j}-1}\left(a_{2^{j}+k} \frac{d \psi_{j, k}(x)}{d x}+\tilde{a}_{2^{j}+k} \frac{d \psi_{j, k}^{*}(x)}{d x}\right)+a_{N / 2} \frac{d \psi_{n-1}(x)}{d x} .
$$

A transformada de Fourier de $g$ será dado por,

$$
\hat{g}_{w}=\sum_{j=0}^{n-2} \sum_{k=0}^{2^{j}-1}\left(a_{2^{j}+k}\left(\frac{d \widehat{\psi_{j, k}(x)}}{d x}\right)_{w}+\tilde{a}_{2^{j}+k}\left(\frac{d \widehat{\psi_{j, k}^{*}(x)}}{d x}\right)_{w}\right)+a_{N / 2}\left(\frac{d \widehat{\psi_{n-1}(x)}}{d x}\right)_{w}
$$

onde,

$$
\left(\frac{d \widehat{\psi_{j, k}(x)}}{d x}\right)_{w}= \begin{cases}\frac{2 \pi i w}{2^{j / 2}} e^{-2 \pi i w \frac{k}{2^{j}}} & \text { quando } w=2^{j}, \ldots, 2^{j+1}-1 \\ 0 & \text { caso contrário }\end{cases}
$$

e

$$
\left(\frac{d \widehat{\psi_{n-1}(x)}}{d x}\right)_{w}= \begin{cases}-N \pi i & \text { quando } w=-\frac{N}{2} \\ 0 & \text { caso contrário. }\end{cases}
$$

Com isso temos que,

$$
\begin{aligned}
\hat{g}_{0} & =0 \\
\hat{g}_{w} & =\frac{2 \pi i w}{2^{j / 2}} \sum_{k=0}^{2^{j}-1} a_{2^{j}+k} e^{-2 \pi i w \frac{k}{2^{j}}}, \quad w=2^{j}, \ldots, 2^{j+1}-1, \\
\hat{g}_{w} & =\hat{g}_{-w}^{*}, \quad w=-N / 2+1, \ldots,-1 \\
\hat{g}_{-N / 2} & =-N \pi i a_{N / 2} .
\end{aligned}
$$


Podemos obter os coeficientes de Fourier de $g$ para os números de onda $2^{j}, \ldots, 2^{j+1}-1$, utilizando transformada de fourier discreta, dada na definição 1.2 .11 .

$$
\hat{g}_{w}=2 \pi i w 2^{j / 2}\left(\left[(-1)^{k} \widehat{\left.a_{2^{j}+k}\right]_{k}}=0, . .2^{j}-1\right)_{w} .\right.
$$

Se são dados os coeficientes de ondaletas harmônicas $a_{2^{j}+k}$, então pode-se calcular os valores de $\hat{g}$, e então calcular os valores de $g$, na malha, com uma transformada de Fourier. O inverso pode ser feito de forma análoga. Se fizermos todas as transformadas, inversas e diretas, de forma rápida (FFTs), então temos um algoritmo rápido para calcular a derivada de uma função a partir de seus coeficientes de ondaletas harmônicas. 


\section{Referências Bibliográficas}

ALPERT, B.; BEYLKIN, G.; GINES, D.; VOZOVOI, L. Adaptive solution of partial differential equations in multiwavelet bases. J. Comput. Phys., v. 182, n. 1, p. 149-190, 2002.

AMARATUNGA, K.; WILLIAMS, J. R.; QIAN, S.; WEISS, J. Wavelet-galerkin solutions for one-dimensional partial differential equations. International Journal for Numerical Methods in Engineering, v. 37, n. 16, p. 2703-2716, 1994.

BACRY, E.; MALLAT, S.; PAPANICOLAOU, G. A wavelet based space-time adaptive numerical method for partial differential equation. Mathematical Modelling and Numerical Analysis, v. 26, n. 7 , p. $793,1992$.

BENEDETTO, J.; FRAZIER, M. Wavelets: Mathematics and Applications. Boca Raton: CRC Press, 1994.

BERTOLUZZA, S. Wavelets and wavelet-like techniques in the numerical solution of PDE. Bellaterra - Barcelona, November 2007.

BERTOLUZZA, S.; NALDI, G. A wavelet collocation method for the numerical solution of partial differential equations. Applied and Computational Harmonic Analysis, v. 3, n. 1, p. 1-9, January 1996.

BESORA, J. Galerkin Wavelet Method for Global Waves in 1D. Dissertação (Master's Degree) - KTH Numerical Analysis and Computer Science, Stockholm, Sweden, 2004. http://www.nada.kth.se/utbildning/grukth/exjobb/rapportlistor/2004/rapporter04/ besora_jordi_04091.pdf, (19/01/2009).

BEYLKIN, G.; KEISER, J. M. On the adaptive numerical solution of nonlinear partial differential equations in wavelet bases. J. Comput. Phys., v. 132, n. 2, p. 233-259, 1997.

BIFERALE, L. Shell models of energy cascade in turbulence. In: Annual review of fluid mechanics, Vol. 35. [S.l.: s.n.], 2003.

BOYD, J. P. Chebyshev and Fourier Spectral Methods. University of Michigan: Dover Publications Inc, Mineola, New York, 1999.

CAI, W.; WANG, J. Adaptive multiresolution collocation methods for initial boundary value problems of nonlinear pdes. SIAM J. Numer. Anal., v. 33, n. 3, 1996.

CANUTO, C.; HUSSAINI, M. Y.; QUARTERONI, A.; ZANG, T. A. Spectral methods in fluid dynamics. New York: Springer-Verlag, 1988. xiv+557 p. (Springer Series in Computational Physics).

CATTANI, C. Harmonic wavelet solutions of the schrodinger equation. International Journal of Fluid Mechanics Research, v. 30, n. 5, p. 1-10, 2003. 
. Harmonic wavelets towards solution of nonlinear PDE. Computers and Mathematics with Applications, v. 50, n. 8-9, p. 1191-1210, July 2005.

. Harmonic wavelet solution of poisson's problem. Balkan Journal of Geometry and Its Applications, v. 13, n. 1, p. 27-37, 2008.

CHUI, C. K. An introduction to wavelets. Boston, MA: Academic Press Inc., 1992. (Wavelet Analysis and its Applications, v. 1).

DAUBECHIES, I. Ten lectures on wavelets. Philadelphia, PA: Society for Industrial and Applied Mathematics (SIAM), 1992. xx+357 p. (CBMS-NSF Regional Conference Series in Applied Mathematics, v. 61).

DOMINGUES, M. O. Análise Wavelet na Simulação Numérica de Equações Diferenciais Parciais com Adaptabilidade Espacial. Tese (Doutorado) — Universidade Estadual de Campinas, UNICAMP, Brasil, 2001.

DOMINGUES, M. O.; GOMES, S. M.; ROUSSEL, O.; SCHNEIDER, K. An adaptive multiresolution scheme with local time stepping for evolutionary pdes. J. Comput. Phys., v. 227, n. 8, p. 3758-3780, 2008.

EGGERS, J.; GROSSMAN, S. Anomalous turbulent velocity scaling from the Navier-Stokes equation. Physics Letters A, v. 156, p. 444-449, jul. 1991.

ELIASEN, E.; MACHENHAUER, B.; RASMUSSEN, E. On a numerical method for integration of the hydrodynamical equations with a spectral representation of the horizontal fields. Rep. No. 2, Institut for Teoretisk Meteorologi, Köbenhavns Universitet, Denmark, 1970.

FRAZIER, M. W. An introduction to wavelets through linear algebra. New York: Springer-Verlag, 1999. xvi+501 p. (Undergraduate Texts in Mathematics).

FRIERSON, D. M. W.; MAJDA, A. J.; PAULUIS, O. M. Large scale dynamics of precipitation fronts in the tropical atmosphere: a novel relaxation limit. Commun. Math. Sci., v. 2, n. 4, p. 591-626, 2004.

GOEDECKER, S.; IVANOV, O. Solution of Multiscale Partial Differential Equations Using Wavelets. Computers in Physics, v. 12, p. 548-555, nov. 1998.

GOMES, S. M.; CORTINA, E. Convergence estimates for the wavelet Galerkin method. SIAM Journal on Numerical Analysis, v. 33, n. 1, p. 149-161, February 1996.

GOTTLIEB, D.; ORSZAG, S. A. Numerical analysis of spectral methods: theory and applications. Philadelphia, Pa.: Society for Industrial and Applied Mathematics, 1977. v+172 p. CBMS-NSF Regional Conference Series in Applied Mathematics, No. 26.

HAAR, A. Zur Theorie der orthogonalen Funktionensysteme. Math. Ann., v. 71, n. 1, p. 38-53, 1911.

HO, S. L.; YANG, S. Y. Wavelet-galerkin method for solving parabolic equations in finite domains. Finite Elem. Anal. Des., v. 37, n. 12, p. 1023-1037, 2001.

HOLTON, J. R. An introduction to dynamic meteorology. 4rd. ed. San Diego: Elsevier Academic Press, 2004. 
JAFFARD, S.; LAURENÇOT, P. Orthonormal wavelets, analysis of operators, and applications to numerical analysis. In: CHUI, C. K. (Ed.). Wavelets - A tutorial in theory and applications. Texas, USA: Academic Press, 1992. p. 543-601.

KHOUIDER, B.; MAJDA, A. J. A non-oscillatory balanced scheme for an idealized tropical climate model- part 1:algorithm and validation. Theoretical and Computational Fluid Dynamics, 2005.

KOZAKEVICIUS, A. d. J. Wavelets Interpolatórias como Ferramenta para Resolução Adaptativa de EDPs Hiperbólicas. Tese (Doutorado) - Instituto de Matemática e Estatística, Universidade de São Paulo, USP, São Paulo, Brasil, 2001.

LAZAAR, S.; PONENTI, P.; LIANDRAT, J.; TCHAMITCHIAN, P. Wavelet algorithms for numerical resolution of partial differential equations. Comput. Methods Appl. Mech. Engrg., v. 116, n. 1-4, p. 309-314, 1994.

LEPIK, Ü. Numerical solution of evolution equations by the Haar wavelet method. Appl. Math. Comput., v. 185, n. 1, p. 695-704, 2007.

LIN, E. B.; ZHOU, X. Connection coefficients on an interval and wavelet solutions of Burgers equation. J. Comput. Appl. Math., v. 135, n. 1, p. 63-78, 2001.

LIU, B. Adaptive harmonic wavelet transform with applications in vibration analysis. Journal of sound and vibration, v. 262, n. 1, p. 45, 2003.

LOPES, E. P.; MATTOS, J. R. L. de. A wavelet Galerkin method applied to partial differential equations with variable coefficients. In: Proceedings of the Fifth Mississippi State Conference on Differential Equations and Computational Simulations. San Marcos, TX: Southwest Texas State Univ., 2003. (Electron. J. Differ. Equ. Conf., v. 10), p. 211-225 (electronic).

MACHENHAUER, B. The spectral method. In: WMO/ICSU. Numerical Methods used in Atmospheric Models. Geneva: GARP Publlication, 1979. (17, v. 2), p. 226-251.

MADAY, Y.; PERRIER, V.; RAVEL, J. Adaptivité dynamique sur bases d'ondelettes pour l'approximation d'équations aux dérivées partielles. C.R. Acad. Sci Paris, v. 312, p. 405-410, 1991.

MALLAT, S. Multiresolution representation and wavelets. Tese (Doutorado) - University of Pennsylvania, Philadelphia, PA, 1988.

MEHRA, M.; KEVLAHAN, N. K. R. An adaptive wavelet collocation method for the solution of partial differential equations on the sphere. J. Comput. Phys., v. 227, n. 11, 2008.

MEYER, Y. Ondelettes et fonctions splines. Séminaire EDP, École Polytechnique, Paris, December 1986.

MORETTIN, P. A. Ondas e Ondaletas: Da análise de fourier à análise de ondaletas. São Paulo, SP, Brasil: EDUSP, 1999. 260 p.

MUNIANDY, S. V.; MOROZ, I. M. Galerkin modelling of the Burgers equation using harmonic wavelets. Physics Letters A, v. 235, n. 4, p. 352-356, November 1997. 
NAIR, M. T. Wavelet-Galerkin Method. December 2004. Lecture Notes, Talk at QIPshort Term Course, Department of Mathematics, IIT Madras, INDIA. Disponível em http://mat.iitm.ac.in/ mtnair/wave.pdf, (17/01/2009).

NEWLAND, D. Harmonic wavelets analysis. Proceedings of the Royal Society of London, Series $A$, v. 443, n. 1917 , p. 203-225, October 1993.

ORZAG, S. A. Comparison of pseudospectral and spectral approximation. Stud. Appl. Math., v. 51, p. 253-259, 1972.

PAULUIS, O.; FRIERSON, D. M. W.; MAJDA, A. J. Precipitation fronts and the reflection and transmission of tropical disturbances. Quarterly Journal of the Royal Meteorological Society, 2008.

PEYRET, R. Spectral Methods for Incompressible Viscous Flow. New York, NY: Springer-Verlag, 2000. (Applied Mathematical Sciences, v. 148).

RUDIN, W. Real and Complex Analysis. International Student Edition: McGraw-Hill Education Europe, 1987.

SAXE, K. Beginning functional analysis. USA: Springer-Verlag, 2000.

SCHULT, R.; WYLD, H. Using wavelets to solve the burgers equation: A comparative study. Physical Review A, v. 46, p. 7953-7958, december 1992.

STECHMANN, S. N.; MAJDA, A. J. The structure of precipitation fronts for the finite relaxation time. Theoretical and Computational Fluid Dynamics, 2006.

STOER, J.; BULIRSCH, R. Introduction to Numerical Analysis. 2. ed. New York: Springer-Verlag, 1992.

VASILYEV, O.; YUEN, D.; PAOLUCCI, S. The solution of pdes using wavelets. Computers in Phys., v. 11, n. 5, p. 429-435, 1997.

VASILYEV, O. V.; KEVLAHAN, N. K.-R. An adaptive multilevel wavelet collocation method for elliptic problems. J. Comput. Phys., v. 206, n. 2, p. 412-431, 2005.

VOIGT, R. G. Spectral Methods for Partial Differential Equations. Philadelphia: SIAM, 1984.

WASSERMANN, A. J. Functional Analysis. University of Cambridge, UK, 1999. http: //www.hep.phys.soton.ac.uk/ g.j.weatherill/lecturenotes/II/FunctionalAnalysis.pdf $(12 / 01 / 2009)$. 